\title{
Space Simulation Testing of the Helicon Double Layer Thruster Prototype
}

\author{
Michael David West \\ BE(Hons) BSc(Adv) Sydney

\begin{abstract}
A thesis submitted for the degree of
Doctor of Philosophy
\end{abstract} \\ of The Australian National University
}

October 2009

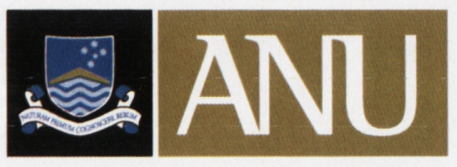

THE AUSTRALIAN NATIONAL UNIVERSITY 
For my parents and grandparents 


\section{Declaration}

This thesis is an account of research undertaken between February 2006 and October 2009 at The Research School of Physics and Engineering, College of Physical Sciences, The Australian National University, Canberra, Australia.

Except where acknowledged in the customary manner, the material presented in this thesis is, to the best of my knowledge, original and has not been submitted in whole or part for a degree in any university.

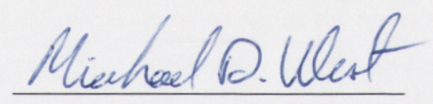

Michael D. West

29th October 2009 


\section{Acknowledgements}

My sincerest thanks and gratitude must first be extended to my supervisors $\mathrm{Dr}$ Christine Charles and Professor Rod Boswell. Thank you both for your guidance, support and encouragement and for having me as part of the family at the Space Plasma, Power and Propulsion Group (SP3). Thank you Christine for giving your time to answer my questions and help me wrestle and persevere with difficult problems. Your enthusiasm for physics is infectious and your skill as an experimentalist and attention to detail are unbounded. It has been a pleasure to learn from you. Thank you Rod for entrusting me with this project and for how you have moulded me as a researcher and as a man. I have always enjoyed our often deep and robust conversations about physics, life, the universe and everything. You have been a great mentor and have taught me the value of Kipling's words in the poem 'If...'.

I am immensely indebted to Peter Alexander, SP3's amazing technician. I am so grateful for all your assistance. You are a true gentleman, whose patience and kindheartedness are rivaled only by your cooking skills. May you remain as healthy as you can, my friend. Thank you to John Wach for patiently teaching me about vacuum technology and for your help with leak testing the space simulation chamber. I also appreciate Dennis Gibson's help and wisdom with all things electronic and RF and wish to thank everyone at The Research School of Physics and Engineering who I have had the pleasure of interacting with.

To the many friends I have made during my time at the ANU, thank you for all the fun memories. In particular, I wish to thank my good friend Dr Cormac Corr. Our fates were intertwined when we both arrived at the ANU on the same day and I am appreciative of your friendship, good humour and help with so many things. I would like to thank Wes Cox for all the interesting and particularly humorous conversations about geography, history and philosophy, Trevor Lafleur for all the fun with puns and Amael Calliard for being such a great office mate. 
Thanks to Orson Sutherland, Ane Aanesland, Albert Meige, Nicolas Balcon and others who helped me so much during my first year or so at SP3 and thank you to Peter Linardakis, Devin Rumdutt and David Pretty for your friendship and always being willing to talk football and sport generally. A big thank you to Jessica Hudspeth, Cameron Samuell, James Dedrick, Sam Dixon and Julia Ling for putting up with me during my final months of writing up. It has also been great to work with many of SP3's international visitors, including Professor Earl Scime, Arjan Flikwert, Boris Legradic, Jan Zanger, Kazunori Takahashi and many others. I made many friends during the ANU Purple Shin football competition; scoring the match-winning goal in the final minute of extra time in the 2007 grand final is a particular highlight!

I am extremely grateful to my close friends Lieutenant Adrian Kriening, Jeffery Candiloro and Andrew Yager for their support and friendship even from afar. Thank you to both Dr Jonathan Clarke and Dr Kimberley Clayfield for your wisdom and for enabling some of my other professional pursuits. Thanks to Andrew Vella and Michael East for the weekly lunches and interesting conversations and a special thank you to Stephen Ryde, who was the science teacher who first inspired me with the wonders of space. Thank you also to Bronwyn Lund for graciously applying your exquisite proof reading skills to this thesis. Any errors in the manuscript are, of course, my own.

I am the person I am because of my parents, my grandparents and my family. Thank you for supporting me in everything I have done. I know that you are proud of me. Thank you for the visits and phone calls and thanks for trying to understand what I have been working on for the past few years. To the joy of my life, my wife Christina, thank you with all my heart for your patience and understanding throughout the past three or more years. I know that this experience has been taxing, and probably at times even more on you than it has been on me. Your love and encouragement have been invaluable and I could not have survived without you. I am looking forward to embarking together on the next stage of our life.

Finally, as I reflect upon the people in my life and the undeserved blessings and opportunities I have been given, the gracious providential work of God is clear. To Jesus Christ - my Creator, my Sustainer, my Lord and my Saviour - thank you for doing for me what no man could. May I always remember and be motivated by that great motto of the Reformation: Soli Deo Gloria. 


\section{Publications}

This thesis has resulted in the following publications in peer reviewed journals:

M. D. West, C. Charles and R. W. Boswell

Testing a Helicon Double Layer Thruster Immersed in a Space-Simulation Chamber Journal of Propulsion and Power, Vol. 24, No. 1, pp. 134-141, 2008.

M. D. West, C. Charles and R. W. Boswell

A high sensitivity momentum flux measuring instrument for plasma thruster exhausts and diffusive plasmas

Review of Scientific Instruments, Vol. 80, No. 053509, pp. 1-9, 2009.

M. D. West, C. Charles and R. W. Boswell

High density mode in xenon produced by a Helicon Double Layer Thruster

Journal of Physics D: Applied Physics, Vol. 42, No. 245201, pp. 1-9, 2009.

M. D. West, C. Charles and R. W. Boswell

Operating RF Antennas in Vacuum: Implications for Ground Testing Plasma Thrusters Journal of Propulsion and Power, in review, 2010. 


\section{Abstract}

A prototype of the Helicon Double Layer Thruster (HDLT), a new magnetoplasma thruster that produces a high velocity collimated ion beam using a current-free double layer, has been tested and characterised for the first time inside a space simulation vacuum chamber. The first experimental evidence of the double layer and associated ion beam with the HDLT prototype immersed in vacuum has been obtained. The plasma and ion beam have been investigated axially and radially using a retarding field energy analyser (RFEA) and a Langmuir probe when operating at low power with argon. The effect of varying the operating pressure and magnetic field on ion beam formation has been characterised and the results compared with recent experiments and theoretical models. A new high sensitivity momentum flux measuring instrument (MFMI) has been developed to measure the thrust imparted by the exhaust of the HDLT prototype. This instrument uses a laser displacement system to measure with high sensitivity the displacement of a target placed in the plasma exhaust. Measurements have been made with the MFMI under various operating conditions and provide insights into the behaviour of the plasma.

Several challenges associated with operating the radio frequency (RF) antenna of the HDLT prototype immersed in vacuum have been identified. The installation of a blocking capacitor in the matching box has mitigated some of the issues and enabled the operation of the HDLT prototype at higher RF powers. When using xenon and increasing the RF power applied to the HDLT prototype, a new high density 'blue' mode has been observed. This mode occurs when the plasma source transitions from a capacitively coupled to what may be a helicon wavesustained mode. The mode transition is accompanied by a significant increase in the plasma density and the appearance of a bright white/blue plasma. The mode has been investigated with the RFEA, Langmuir probe, MFMI and spectroscopically and it has been shown that the thrust produced by the HDLT prototype increases in this mode. 


\section{Contents}

$\begin{array}{ll}\text { Declaration } & \text { i }\end{array}$

Acknowledgements $\quad$ ii

$\begin{array}{ll}\text { Publications } & \text { iv }\end{array}$

$\begin{array}{lll}\text { Abstract } & \text { v }\end{array}$

List of Figures $\quad$ xi

List of Tables $\quad$ Xv

1 Introduction 1

1.1 Principles of Spacecraft Propulsion . . . . . . . . . . . . . . 1

1.2 Chemical Propulsion .................... 2

1.3 Electric Propulsion .................... 4

1.3.1 Types and Classification ............. 5

1.3.2 Applications and Missions ............. 6

1.4 Plasmas for Spacecraft Propulsion . . . . . . . . . . . . 8

1.4.1 Plasma Production ................ 8

1.4.2 Ionisation Energy and Propellant Selection . . . . . . . . 9

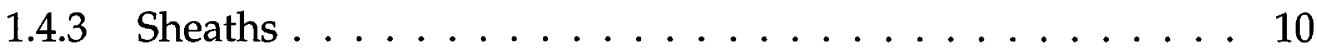


1.5 Current-Free Double Layers . . . . . . . . . . . . . . 11

1.5.1 Recent Experimental Work . . . . . . . . . . . . 13

1.5.2 Theoretical and Simulation Work . . . . . . . . . . . . 15

1.6 The Helicon Double Layer Thruster Concept . . . . . . . . . . 17

1.6.1 Advantages and Applications . . . . . . . . . . . 17

1.6.2 Testing Campaign at ESTEC . . . . . . . . . . . . . 19

1.6.3 Unresolved Questions . . . . . . . . . . . . . . 19

1.7 Thesis Scope . . . . . . . . . . . . . . . . . . 20

2 Experimental Apparatus and Diagnostics 22

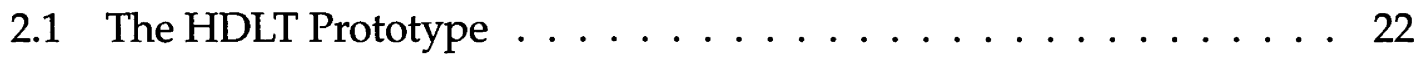

2.2 Space Simulation Chamber $\ldots \ldots \ldots \ldots \ldots \ldots \ldots$

2.3 Radio Frequency Matching Network . . . . . . . . . . . 28

2.4 Magnetic Field Topography $\ldots \ldots \ldots \ldots \ldots$

2.5 Langmuir Probe . . . . . . . . . . . . . . . 31

2.5 .1 Principles of Operation $\ldots \ldots \ldots$. . . . . . . 31

2.5.2 Probe Design and Installation . . . . . . . . . . . . 33

2.5.3 Probe Operation and Data Analysis . . . . . . . . . 34

2.6 Retarding Field Energy Analyser . . . . . . . . . . . . 35

2.6.1 Principles of Operation $\ldots \ldots \ldots \ldots$

2.6.2 Probe Design and Installation . . . . . . . . . . . . 35

2.6 .3 Probe Operation $\ldots \ldots \ldots \ldots \ldots \ldots \ldots$

2.6 .4 Data Analysis . . . . . . . . . . . . . . . . 38

2.7 High Voltage Probe . . . . . . . . . . . . . . . . . 41

2.8 USB Spectrometer . . . . . . . . . . . . . . . . . 42

2.9 Digital Oscilloscope . . . . . . . . . . . . . . . . . . 42 
3.1 Principles of Thrust Measurement $\ldots \ldots \ldots \ldots$. . . . . . 43

3.2 Thrust Measurement Techniques . . . . . . . . . . . . 44

3.2.1 Challenges Associated with the HDLT Prototype . . . . . 46

3.3 Previous Momentum Flux Instruments $\ldots \ldots \ldots$

3.4 Momentum Flux Measuring Instrument Design $\ldots \ldots$. . . . . 50

3.4.1 Instrument Construction . . . . . . . . . . . . . 52

3.4.2 Installation and Laser Measurement System . . . . . . 53

3.4.3 Minimising Unwanted Perturbations . . . . . . . . . . 54

3.5 Calibration . . . . . . . . . . . . . . . . 54

3.6 Methodology and Data Analysis . . . . . . . . . . 58

3.7 Response to Cold Gas Flow . . . . . . . . . . . . . . . 59

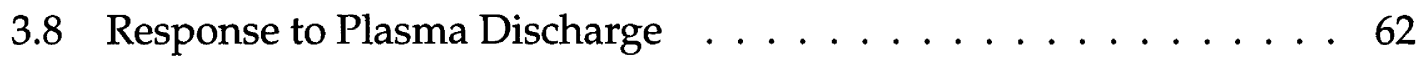

3.8.1 Plasma Parameters Determined from Diagnostics . . . . . 64

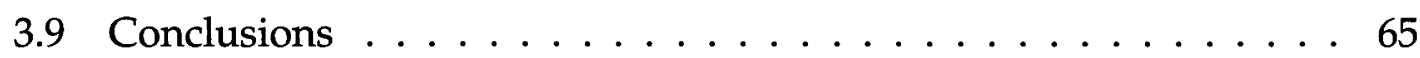

4 Low Power Characterisation of the HDLT Prototype 67

4.1 Ion Beam Detection $\ldots \ldots \ldots \ldots$. . . . . . . . 68

4.2 Axial Plasma Characterisation . . . . . . . . . . . . . . 70

4.2.1 Axial Plasma and Beam Potential Profile . . . . . . . . 70

4.2.2 Axial Density Profile . . . . . . . . . . . . . . . 72

4.2.3 Electron Temperature Downstream _. . . . . . . . . 73

4.3 Radial Plasma Characterisation . . . . . . . . . . . . . . . 75

4.3.1 Radial Plasma and Beam Potential Profile . . . . . . . . . 76

4.3.2 Radial Ion Flux Profile . . . . . . . . . . . . . . . 76

4.3.3 Radial Expansion and Electron Temperature . . . . . . . 79 
4.4 Double Layer Characterisation $\ldots \ldots \ldots \ldots$

4.5 Pressure Dependence . . . . . . . . . . . . . . . . . . 81

4.6 Magnetic Field Dependence . . . . . . . . . . . . . . . 83

4.7 Effect of Varying Physical Configuration . . . . . . . . . . 86

4.8 Ion Beam Flux and Plasma Detachment $\ldots \ldots \ldots \ldots$. . . . 87

4.9 MFMI Measurements Downstream . . . . . . . . . . . . . . . . 89

4.9.1 Varying Propellant Flow Rate . . . . . . . . . . . . . . 91

4.9.2 Varying Magnetic Field Strength . . . . . . . . . . . 94

4.9 .3 Varying RF Power $\ldots \ldots \ldots \ldots$

4.10 Measurements with Xenon . . . . . . . . . . . . . . . . 97

4.11 Estimate of Exhaust Velocity and Thrust . . . . . . . . . . 98

4.12 Conclusions . . . . . . . . . . . . . . . . . . . 101

5 Operating RF Antennas Immersed in a Vacuum 103

5.1 Parasitic Plasma Formation . . . . . . . . . . . . . . . . . . 104

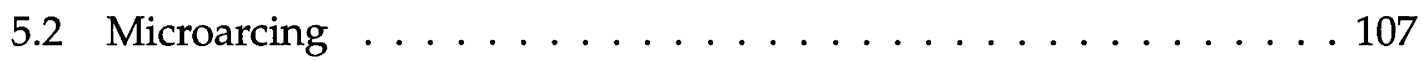

5.3 Possible Solutions . . . . . . . . . . . . . . . . . . . . . . . . . 109

5.4 Matching Box Modifications and Consequences $\ldots \ldots \ldots \ldots 111$

5.5 Thermal Loading . . . . . . . . . . . . . . . . . . 115

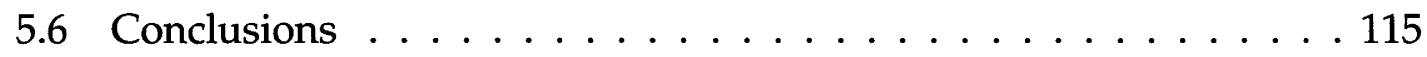

6 High Power Characterisation of the HDLT Prototype 118

6.1 Operating Modes in RF Discharges . . . . . . . . . . . . 118

6.2 High Density Mode in Xenon . . . . . . . . . . . . . . . . 119

6.3 Source Characterisation $\ldots \ldots \ldots \ldots \ldots \ldots$

6.4 Pressure Dependance . . . . . . . . . . . . . . . . . . . . 124

6.5 Radial Profile Inside HDLT Source . . . . . . . . . . . . . . 125 
6.6 Investigation of Matching Box Circuit $\ldots \ldots \ldots \ldots \ldots$

6.7 Downstream Measurements . . . . . . . . . . . . . . . . 128

6.8 MFMI Measurements with Xenon at High Power . . . . . . . . . . 131

6.9 Estimate of Thrust . . . . . . . . . . . . . . . . . 134

6.10 Spectroscopic Studies . . . . . . . . . . . . . . . . 135

6.11 Conclusions . . . . . . . . . . . . . . . 138

$\begin{array}{lll}7 \text { Conclusion } & 141\end{array}$

7.1 Recommendations and Future Work . . . . . . . . . . 143

$\begin{array}{ll}\text { Bibliography } & 145\end{array}$ 


\section{List of Figures}

1.1 The potential profile of a wall sheath. . . . . . . . . . 11

1.2 The potential profile for a double layer in an expanding plasma. . . 12

1.3 The Chi Kung experiment and key results. . . . . . . . . . . . . 14

2.1 The Helicon Double Layer Thruster prototype. . . . . . . . . . . 23

2.2 A cut away view of the HDLT prototype . . . . . . . . . . 24

2.3 The double-saddle field antenna used in the HDLT prototype. . . . 24

2.4 The HDLT prototype integrated into the space simulation chamber. 26

2.5 Images of the space simulation chamber $\ldots \ldots \ldots \ldots \ldots$

2.6 A schematic of the $L$ impedance matching network. . . . . . . . . . 29

2.7 The electrical system for the HDLT prototype . . . . . . . . . . . . 29

2.8 The magnetic field topography $\ldots \ldots \ldots \ldots \ldots \ldots$

2.9 A typical Langmuir probe current-voltage characteristic. . . . . . 32

2.10 A cross sectional view of the dogleg Langmuir probe. . . . . . . . 34

2.11 A cross sectional and exploded view of the RFEA probe. . . . . . 36

2.12 The RFEA biasing circuit and potentials . . . . . . . . . . . 38

2.13 Normalised $I_{c}$ vs $V_{d}$ curve and IEDF $\ldots \ldots \ldots \ldots$

2.14 A typical voltage trace measured by the high voltage probe. . . . . 41

3.1 The three most common thrust balance configurations. . . . . . . 45 
3.2 The momentum flux instrument developed for VASIMR. . . . . . . 49

3.3 A compound pendulum with an arbitrary distribution of mass. . . 51

3.4 Construction of the MFMI. . . . . . . . . . . . . . . 52

3.5 The MFMI installed in the space simulation chamber. . . . . . . . 53

3.6 Free body diagrams for MFMI calibration. . . . . . . . . . . 55

3.7 Typical calibration curves for the MFMI . . . . . . . . . . . 57

3.8 The data outputted from the laser displacement sensor. . . . . . . 59

3.9 The forces acting on the MFMI target from cold gas flow. . . . . . 60

3.10 Force on the target from the propellant flow . . . . . . . . . . 61

3.11 Forces acting on the MFMI target from the plasma . . . . . . . . 63

4.1 Measurements with the RFEA placed upstream at $\mathrm{z}=-2 \mathrm{~cm} \ldots .69$

4.2 Measurements with the RFEA placed downstream at $\mathrm{z}=13 \mathrm{~cm} \quad \ldots 69$

4.3 Plasma and ion beam potential vs axial position. . . . . . . . 71

4.4 Plasma potential vs axial position at different pressures. . . . . . 72

4.5 Plasma density vs axial position $\ldots \ldots \ldots \ldots \ldots \ldots$

$4.6 T_{e}$ determined from the Langmuir probe $\mathrm{I}-\mathrm{V}$ curve $\ldots \ldots \ldots$

4.7 Plasma density as function of plasma potential . . . . . . . . 75

4.8 Radial profile of the IEDFs measured downstream with the RFEA. . 77

4.9 Radial ion flux profile measured with the RFEA. . . . . . . . 78

4.10 Radial ion beam flux profile measured with the RFEA. . . . . . 79

4.11 Radial total ion flux vs the plasma potential . . . . . . . . . . 80

4.12 Double layer strength vs pressure. . . . . . . . . . . . . 82

4.13 Ion beam and plasma potential vs solenoid current. . . . . . . . 84

4.14 Radial potential profiles for different magnetic field configurations 85

4.15 Radial ion flux profiles for different magnetic field configurations. . 85

4.16 Plasma density with HDLT source tube retracted. . . . . . . . 87 
4.17 Ion beam flux vs axial position. . . . . . . . . . . . . 88

4.18 The RFEA installed in front of the MFMI target. . . . . . . . . . 90

4.19 Force on MFMI target vs flow rate. . . . . . . . . . . . . . . 92

4.20 Plasma parameters at MFMI location vs flow rate. . . . . . . . 93

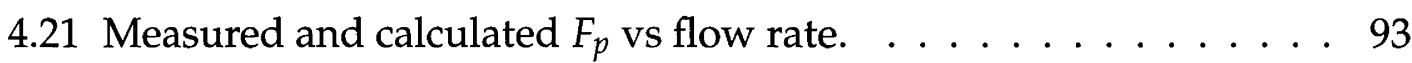

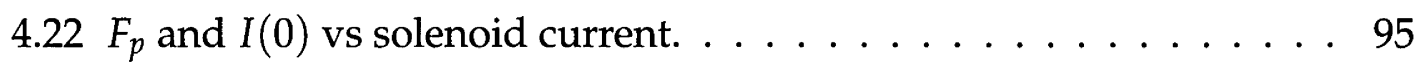

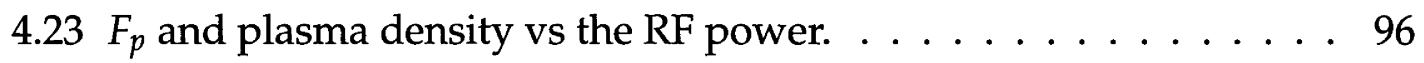

4.24 Double layer strength vs pressure for xenon $\ldots \ldots \ldots$. . . . 98

4.25 The calculated exhaust velocity vs pressure for argon and xenon . . 99

5.1 The plasma forming around the antenna. . . . . . . . . . . 104

5.2 Images of the damage caused by parasitic plasma formation. . . . 105

5.3 The density versus the axial position at $\mathrm{r}=32 \mathrm{~cm} \ldots \ldots \ldots 107$

5.4 The voltage distributions of the immersed antenna system. . . . . 108

5.5 The floating potential vs time during a microarc event. . . . . . . 109

5.6 The modified matching box circuit with the blocking capacitor. . . 112

5.7 The blocking capacitor installed inside the matching box. . . . . . 112

5.8 The antenna bias voltage vs RF power. . . . . . . . . . . . 113

5.9 A short circuit on the antenna strap caused by sputtered silver. . . 114

5.10 The repaired antenna with ceramic spacers installed. . . . . . . 114

5.11 The broken HDLT source tube. . . . . . . . . . . . . 116

6.1 Mode transition power vs pressure at ANU and ESTEC. . . . . . . . 121

6.2 HDLT source density vs RF power with hysteresis. . . . . . . . 122

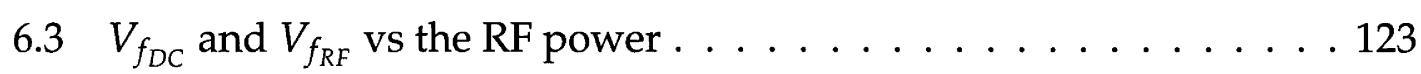

6.4 The density vs RF power at various pressures. . . . . . . . . . 124

6.5 The density profiles vs radial position inside the source. . . . . . . 125 
6.6 Peak to peak voltage vs RF power. . . . . . . . . . . . . . . 127

6.7 Quality factor vs RF power. . . . . . . . . . . . . . . . . . 127

6.8 The density vs axial position in xenon. . . . . . . . . . . . . . 129

6.9 The plasma potential vs axial position in the high density mode. . . 129

6.10 The density vs plasma potential in the high density mode. . . . . 130

6.11 Natural logarithm of the electron current measured downstream. . 131

$6.12 F_{p}$ in xenon vs RF power with the $50 \mathrm{~mm}$ diameter MFMI target. . . 132

6.13 Density in front and behind the $50 \mathrm{~mm}$ diameter MFMI target. . . . 132

6.14 The radial density profile measured in front of the MFMI target. . . 133

6.15 The HDLT source in the capacitive and high density modes. . . . 136

6.16 Emission spectrum for xenon in the HDLT source. . . . . . . . 137

6.17 The emission intensity in the HDLT source vs RF power . . . . . . 137

6.18 Emission spectrum for xenon in the HDLT exhaust. . . . . . . 138

6.19 The emission intensity in the HDLT exhaust vs RF power . . . . . 139 


\section{List of Tables}

1.1 Examples of various electric propulsion systems $\ldots \ldots \ldots \ldots$

1.2 Ionisation energy and molecular mass for various propellants . . 10

1.3 Recent experiments in low pressure expanding magnetised plasmas 16

3.1 Details for the calibration of the two MFMI configurations used. . . 56 


\section{Introduction}

\subsection{Principles of Spacecraft Propulsion}

In their most basic form, all spacecraft propulsion systems propel themselves by emitting a jet of matter. The momentum carried away by the jet results in a force, acting to accelerate the spacecraft in the direction opposite to that of the jet. This is in accordance with Newton's Third Law of Motion, which states that

'For every action there is always an equal and opposite reaction'

A spacecraft experiencing no net forces can only attain acceleration from the discharge of propellant mass and since momentum is conserved, the equation of motion of the spacecraft is

$$
m \dot{v}=\dot{m}_{p} v_{e x}
$$

where $m$ is the mass of the spacecraft, $\dot{v}$ is the acceleration of the spacecraft, $\dot{m}_{p}$ is the rate of change of the spacecraft mass due to the expulsion of propellant and $v_{e x}$ is the exhaust velocity of the ejected propellant relative to the spacecraft.

If the exhaust velocity is constant over the acceleration period, the spacecraft achieves an incremental increase in its velocity, $\Delta v$, which is related to the exhaust velocity and the amount of propellant mass expelled by

$$
\Delta v=v_{e x} \ln \frac{m_{d}+m_{p}}{m_{d}}
$$

where $m_{p}$ is the mass of the propellant, $m_{d}$ is the delivered spacecraft mass at the end of the acceleration period and therefore $m_{d}+m_{p}$ is the initial spacecraft mass. Equation 1.2 is known as the Ideal Rocket Equation and its initial derivation is generally attributed to Konstantin Tsiolkovsky, who first published it in 
1903 (Tsiolkovsky, 1903). The deliverable mass fraction of the spacecraft $\frac{m_{d}}{m_{d}+m_{p}}$ can also be found by rearranging Equation 1.2 such that

$$
\frac{m_{d}}{m_{d}+m_{p}}=e^{-\Delta v / v_{e x}}
$$

In practice, $\Delta v$ is considered a generalised 'characteristic velocity increment' because a spacecraft experiences significant gravitational and drag forces during flight that add additional terms to Equation 1.1. Irrespective of this, Equation 1.3 is still valid and $\Delta v$ is regarded as an indication of the energetic difficulty of a particular spacecraft mission or manoeuvre. Equation 1.2 highlights that the final velocity of the spacecraft depends only on the deliverable mass fraction and the exhaust velocity, not on the size of the propulsion system, the time it is employed for, the amount of thrust produced or any other parameter (Sutton and Biblarz, 2001).

However, there are several parameters that are useful for classifying and comparing different spacecraft propulsion systems, most notably the specific impulse and the thrust. The specific impulse, $I_{s p}$, is the total impulse per unit weight of propellant and is related to the exhaust velocity such that

$$
I_{s p}=\frac{v_{e x}}{g_{0}}
$$

where $g_{0}$ is the acceleration due to gravity at sea-level, which equals $9.81 \mathrm{~ms}^{-2}$. $I_{s p}$ has historically been used to measure performance, with a higher number meaning better performance.

As per Newton's Second Law of Motion, the force produced by a spacecraft propulsion system, known as the thrust $T$, equals the rate of change of the mass of the propellant times the exhaust velocity such that

$$
T=\dot{m}_{p} v_{e x}
$$

and has SI units of kg.ms ${ }^{-2}$ or Newtons, N.

\subsection{Chemical Propulsion}

The most widely used form of spacecraft propulsion is chemical propulsion. Chemical propulsion systems generate thrust via the combustion of a propellant inside a chamber, which is then expanded through a nozzle and ejected as hot gas. Both solid and liquid fueled rockets typically produce thrust on the order of 
$10^{6} \mathrm{~N}$ (Turner, 2005). The exhaust velocity is generally dictated by the molecular and chemical properties of the propellant used and the pressure and temperature that the walls can withstand. It is therefore limited to about $3-5 \mathrm{kms}^{-1}$ by the combustion reaction energies and heat transfer tolerances of the propellants (Sutton and Biblarz, 2001).

Chemical propulsion systems have been employed for all types of mission scenarios ranging from launch from the Earth's surface to interplanetary and orbit station-keeping manoeuvres. Chemical rockets are still required for reaching Earth orbit as high thrust levels are needed to escape the gravity well of the Earth. However, this is expensive; it currently costs approximately AUS $\$ 10,000$ per kilogram to launch a spacecraft into low Earth orbit (LEO) and approximately AUS\$50,000 per kilogram to launch a spacecraft into an Earth escaping orbit, say towards the Moon or Mars (Buursink, 1998). Since the mass of propellant used in a typical communications satellite, for example, can be close to $20 \%$ of the total spacecraft mass delivered to orbit (Larson and Wertz, 1999), any reductions in propellant mass can result in significant cost savings and increase the amount of useful payload that can be deployed.

Fortunately, as shown in Equation 1.3, the deliverable mass fraction is an exponential function of the velocity ratio and therefore small improvements in the exhaust velocity can lead to large increases in the deliverable mass fraction for a given mission. This is particularly true of ambitious interplanetary missions that have a high $\Delta v$ and therefore require exhaust velocities higher than $10 \mathrm{kms}^{-1}$ to be feasible (Frisbee, 2003). If Equation 1.3 is considered in terms of how much propellant is required for a given mission, such that

$$
m_{p}=m_{d}\left[e^{\Delta v / v_{e x}}-1\right]
$$

the dramatic benefits gained by high exhaust velocities can be quantified.

Consider as an example the recently launched $D A W N$ mission, which has a delivered payload of $800 \mathrm{~kg}$. In order to reach, from Earth orbit, the main belt asteroids of Ceres and Vesta, a $\Delta v$ of about $11 \mathrm{kms}^{-1}$ is required (Rayman et al., 2006). If a chemical propulsion system with an exhaust velocity of $3 \mathrm{kms}^{-1}$ was used for the in-space manoeuvres, using Equation 1.6, approximately $30,500 \mathrm{~kg}$ of propellant would be required. This is excluding the mass of the propellant and the propulsion system required to get the spacecraft from the Earth's surface to LEO. When using chemical propulsion, only $2.5 \%$ of the total spacecraft mass delivered to LEO would be useful payload. If a propulsion system with an exhaust velocity of $30 \mathrm{kms}^{-1}$ was used, from Equation 1.6, only $355 \mathrm{~kg}$ of propellant would be required and about $70 \%$ of the spacecraft mass would be useful 
payload. As a result, the DAWN mission, along with many other types of missions, is not feasible with chemical propulsion alone. To execute these missions, propulsion systems with much higher exhaust velocities are preferred, which is the domain of electric propulsion.

\subsection{Electric Propulsion}

First envisaged by Tsiolkovsky in 1911, electric propulsion accelerates gases for propulsion by electrical heating and/or by electric and magnetic body forces (Jahn, 1968). This results in significantly higher exhaust velocities, and hence $I_{s p}$, than is possible from chemical reactions. Thrust is mainly obtained from the ejection of ionised particles, or ions, and neutrals accelerated by collisions. The thrust range is very low because of the low densities involved and therefore is typically on the order of $0.1-100 \mathrm{mN}$.

The thrust produced by these ions, from Equation 1.5, is

$$
T_{i}=\dot{m}_{i} v_{i_{e x}}
$$

where $\dot{m}_{i}$ is the mass flow rate of ionised propellant and $v_{i_{e x}}$ is the ion exhaust velocity. The ion exhaust velocity can be expressed, when an ion is accelerated electrostatically by a potential difference, $V$, as

$$
v_{i_{e x}}=\sqrt{\frac{2 q V}{m_{i}}}
$$

where $q$ is the ion charge. Since the ion flow rate equals the ion beam current divided by the charge, $\frac{I_{b}}{q}$, the thrust produced by a monoenergetic, singularly charged $\left(q=e=1.602 \times 10^{-19} \mathrm{C}\right.$ ) ion beam that is accelerated by a potential $V_{b}$ and has zero divergence is

$$
T_{i}=\frac{m_{i} I_{b}}{e} v_{i_{e x}}=\sqrt{\frac{2 m_{i}}{e}} I_{b} \sqrt{V_{b}}
$$

The thrust is therefore proportional to the beam current times the square root of the acceleration voltage. In reality, there is a spread of beam energies produced and $V_{b}$ represents the effective acceleration voltage.

For comparison, the thrust obtained from simply ejecting neutral propellant, $T_{n}$, can be estimated from the 'random' flux across a surface area, $A$. As described 
by Braithwaite (2000), for an isotropic thermal distribution of temperature $T$, the flux of neutral propellant is

$$
\Gamma_{n}=\frac{1}{4} n_{n} v_{n} A
$$

where $n_{n}$ is the neutral gas density in atoms.m $\mathrm{m}^{-3}$ and $v_{n}=\sqrt{\frac{8 k T}{\pi m_{n}}}$ where $m_{n}$ is the mass of the neutral atom and $k$ is the Boltzmann constant. Therefore from Equation 1.5,

$$
T_{n}=\dot{m}_{n} v_{n_{e x}}=\frac{1}{4} n_{n} m_{n} A v_{n}^{2}
$$

where $v_{n_{e x}}$ is the exhaust velocity of the neutrals and equals $v_{n}$. The thrust from neutral propellant alone is typically on the order of $0.1-1 \mathrm{mN}$.

Unlike chemical propulsion systems, which have their energy source stored in the propellant as chemical bonds, electric propulsion systems require an external power source to provide the electrical energy for producing and accelerating ions. This power supply introduces an additional mass to a spacecraft, which typically increases linearly with power supply output. The efficiency with which electrical energy is converted to thrust must also be considered. These terms complicate the performance parameters for electric propulsion systems and the details are discussed further by Turner (2005). The consequence is that the exhaust velocity is no longer the sole driver of performance because increasing the exhaust velocity or the mass flow rate requires an increase in the electrical power supplied, which in turn increases the mass of the spacecraft. In reality, exhaust velocities in the range of $10-40 \mathrm{kms}^{-1}$ are optimal for most mission scenarios.

\subsubsection{Types and Classification}

Electric propulsion systems have been under development for more than 70 years and several hundred systems have flown in space. Historically, these systems have been divided into three broad categories based on the main acceleration mechanism: electrothermal, electrostatic and electromagnetic (Jahn, 1968). In electrothermal systems, the propellant is heated by an electrical process and then expanded through a nozzle. Examples include arcjets and resistojets. Gridded ion thrusters are the principal electrostatic system, where the propellant is accelerated by the direct application of electrostatic forces to ionised particles. This is achieved by the application of a potential that accelerates the beam of ions. The potential is applied via a series of biased grids and the ejected ion beam is neutralised by an electron source to maintain charge balance. 
Electromagnetic systems accelerate propellant under the combined action of electric and magnetic fields. A myriad of possibilities for electromagnetic acceleration exist and the most common implementations are magnetoplasmadynamic (MPD) thrusters and Hall effect thrusters. The latter are the most commonly used plasma thrusters and more than a hundred have flown in space, mainly aboard spacecraft developed by the Russians, who were the early pioneers of the technology. The Variable Specific Impulse Magnetoplasma Rocket (VASIMR) is a high power propulsion concept that is currently under development. It can also be considered an electromagnetic system as it uses radio frequency electromagnetic fields to ionise propellant and accelerate it through a magnetic nozzle (Chang-Diaz, 2006).

Table 1.1 shows some examples of the different electric propulsion systems and summarises their key performance parameters and other relevant details. More information about these systems and their advantages and disadvantages can be found in the various reviews available (Jahn, 1968; Martinez-Sanchez and Pollard, 1998; Jahn and Choueiri, 2002; Goebel and Katz, 2008; Charles, 2009).

\subsubsection{Applications and Missions}

Electric propulsion can only be used in space because a vacuum is required for the ionisation of propellant at practical power levels. Furthermore, the low thrust range is insufficient to propel a spacecraft from the surface of the Earth to orbit. The most common applications of electric propulsion are satellite station-keeping and orbital manoeuvres. This can include orbit raising and insertion manoeuvres, orbital maintenance such as North-South and East-West attitude control and de-orbiting at the end of a satellite's operational life.

More recently there has been an increased interest in electric propulsion as the primary propulsion for interplanetary probes. A gridded ion thruster was used aboard the US Deep Space 1 mission that was launched in 1998 and completed flybys of Comet Borrelly and the asteroid 9969 Braille. The European SMART 1 mission was launched to the Moon in 2003 and used a Hall effect thruster as the primary propulsion system.

The Hayabusa mission, launched by the Japanese in 2003 to asteroid Itokawa, was equipped with four microwave ion thrusters. In addition to providing primary propulsion, the thrusters doubled as an attitude control system and saved the mission when the reaction control wheels failed. Hayabusa is due to return samples from the asteroid to Earth in 2010. 


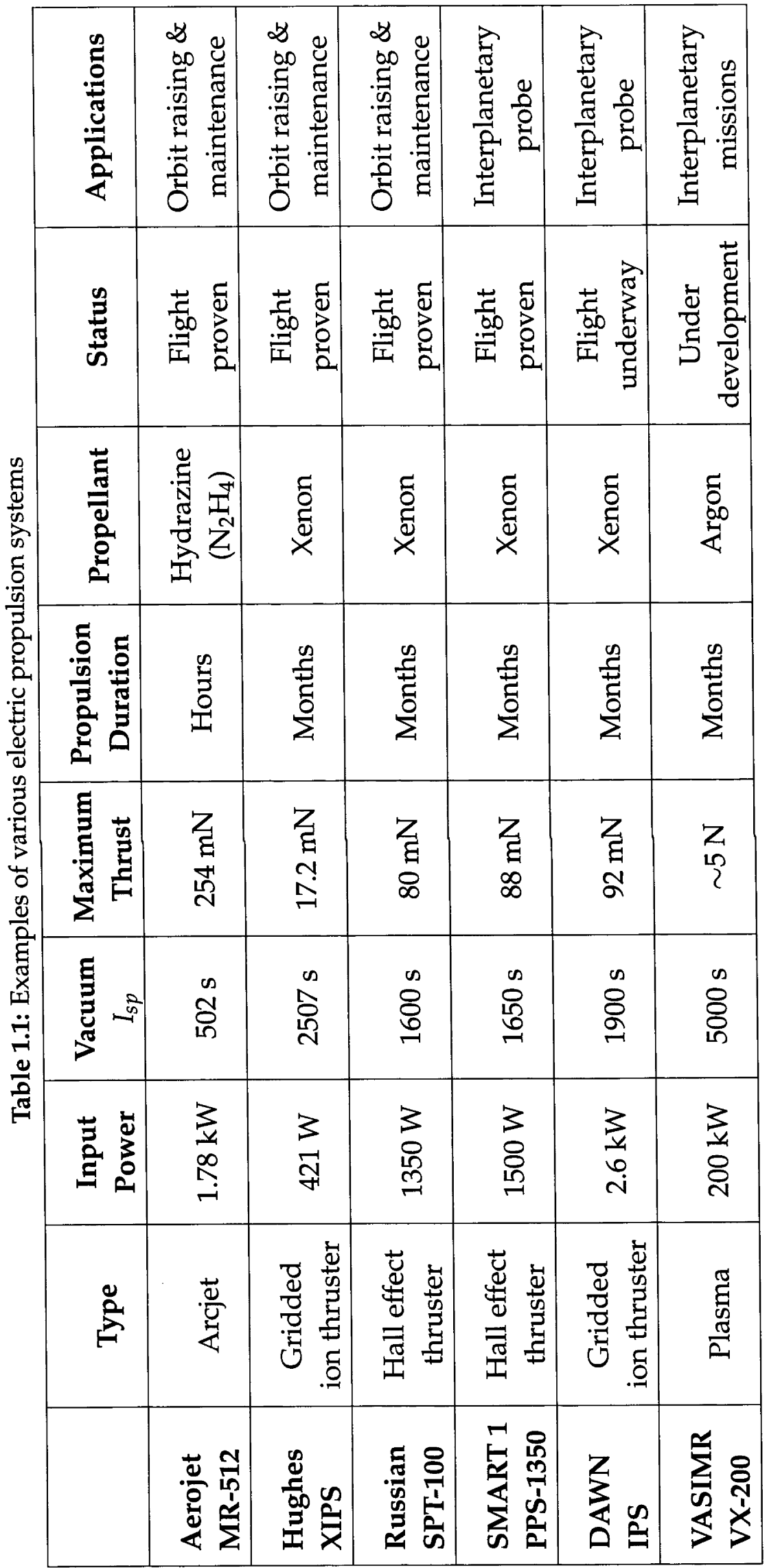


The previously mentioned DAWN mission, launched by the US in 2007, uses three ion thrusters and is currently en route to the main belt asteroids Ceres and Vesta. In 2014 another European mission named BepiColombo will depart for Mercury. This spacecraft will use four ion thrusters for propulsion during the cruise phase of the mission.

Electric propulsion is also often proposed for crewed and cargo missions to the Moon and Mars and robotic missions to the outer solar system (Brophy, 2003; Schmidt et al., 2006). This is motivated by the increase in useful payload that can be delivered. However, the mass of the power supply typically scales with the distance travelled such that a return mission to the outer planets would require about $200 \mathrm{~kW}$, a cargo mission to Mars about 200-600 kW and a crewed Mars mission over $1 \mathrm{MW}$ (Charles, 2009). Work is underway to develop the low mass, high power energy sources required to enable these missions, although progress has been slow to date.

\subsection{Plasmas for Spacecraft Propulsion}

Plasma is generally referred to as the fourth state of matter, after solids, liquids and gases. A plasma is a partially ionised gas, with free particles moving in random directions, that is, on average, electrically neutral (Lieberman and Litchtenberg, 2005). Approximately $99 \%$ of the observable universe is composed of plasma since the interior of stars, gaseous nebulas and much of the interstellar hydrogen are in the plasma state. However, naturally occurring terrestrial plasmas are rare, with examples including the glow of the aurora at high northern and southern latitudes and the bright flash of a lightning bolt. Artificially created plasmas can be found in a fluorescent light tube, a neon sign or in the electric arc of an arc welder.

All of the systems discussed in Section 1.3 use plasma discharges to produce ions for propulsion. Some are true 'plasma thrusters' as they eject plasma to produce thrust, while others create a plasma, extract and eject the ions and then neutralise them with a free stream of electrons further downstream.

\subsubsection{Plasma Production}

Several methods can be used to generate and sustain a plasma, but the application of an electric field is the method of most interest here. When an electric field 
is applied to a neutral gas, which always contains a few free electrons and ions (Lieberman and Litchtenberg, 2005), these free charge carriers are accelerated by the electric field. They then collide with atoms and molecules to create more charged particles that are also under the influence of the electric field. This leads to an avalanche of charge particles, known as 'breakdown', that is eventually balanced by charge carrier losses, so that a steady-state plasma develops.

One way to excite and sustain a plasma discharge is via the application of a highfrequency electromagnetic field. An example is the helicon source, which uses a specially shaped radio frequency (RF) antenna typically driven at $13.56 \mathrm{MHz}$. The antenna is wrapped around a dielectric cylinder and the RF power is coupled to the plasma via the dielectric. Helicon sources can operate in the capacitively coupled, inductively coupled and the wave-sustained mode; the latter requiring the presence of a magnetic field (Boswell, 1970, 1984). These different modes are discussed further in Chapter 6.

\subsubsection{Ionisation Energy and Propellant Selection}

The ionisation energy is a measure of how much work, measured in $\mathrm{eV}$, is required to remove the least bound electron in a single neutral atom or molecule and thereby ionise each neutral particle to create a plasma. The lower the ionisation energy, the easier it is to ionise the neutral gas. In electric propulsion applications, in order to produce the most thrust possible with the least amount of electrical power, the propellant with the lowest ionisation energy and highest molecular mass is optimal. Table 1.2 shows the ionisation energies and molecular masses for various propellant options.

Mercury and caesium are the obvious choices as they have very low ionisation energies and high molecular masses. However, these materials are toxic and reactive metals and early tests revealed that contamination of sensitive spacecraft components and solar arrays is a significant issue (Hall et al., 1970). Therefore, inert gases are the preferred choice. As shown in Table 1.2, neon, argon, krypton and xenon all have similar ionisation energies of about $12-22 \mathrm{eV}$, but xenon has the highest molecular mass. Xenon is therefore the propellant of choice for electric propulsion and has been used on all recent missions. Xenon also has a high liquid density under mild cryogenic storage, which simplifies propellant tank design. 
Table 1.2: Ionisation energy and molecular mass for various propellant options

\begin{tabular}{|l|c|c|}
\hline Propellant & $\begin{array}{c}\text { Ionisation } \\
\text { Energy, V }\end{array}$ & $\begin{array}{c}\text { Molecular } \\
\text { Mass, g.mol }^{-1}\end{array}$ \\
\hline Caesium vapour (Cs) & 3.9 & 132.91 \\
Potassium vapour (K) & 4.3 & 39.10 \\
Mercury vapour (Hg) & 10.4 & 200.59 \\
Xenon (Xe) & 12.1 & 131.29 \\
Krypton (Kr) & 14.0 & 83.79 \\
Argon (Ar) & 15.7 & 39.95 \\
Neon (Ne) & 21.6 & 20.18 \\
Helium (He) & 24.6 & 4.00 \\
\hline
\end{tabular}

\subsubsection{Sheaths}

In general, large electric fields cannot be sustained in a low collisional plasma except at the boundaries, such as at a vacuum chamber wall or around an electrode. In order to balance the flux of the mobile electrons and the less mobile ions, the plasma is joined to these boundaries across thin positively charged layers called sheaths. The sheath forms with a potential, $V_{s}$, such that

$$
V_{s} \sim \frac{k T_{e}}{e} \ln \sqrt{\frac{m_{i}}{2 \pi m_{e}}}
$$

where $k$ is the Boltzmann constant, $T_{e}$ is the electron temperature, $e$ is the electron charge and $m_{i}$ and $m_{e}$ are the positive ion and electron masses, respectively. In argon, the sheath potential is typically $\sim 4.7\left(k T_{e} / e\right)$ (Lieberman and Litchtenberg, 2005).

Near a grounded wall following plasma breakdown, the steady-state potential profile is similar to that shown in Figure 1.1. This potential profile forms an accelerating electric field for ions and a retarding field for the electrons. Since the ion density is much larger than the electron density in the sheath and it therefore contains mostly positive charges, the region is often referred to as a 'single layer' or an 'ion sheath'.

A presheath region also exists, to maintain sheath equilibrium, that accelerates the ions to the Bohm velocity via a potential drop of approximately $0.5-1\left(k T_{e} / e\right)$ (Lieberman and Litchtenberg, 2005). The Bohm velocity is also known as the 


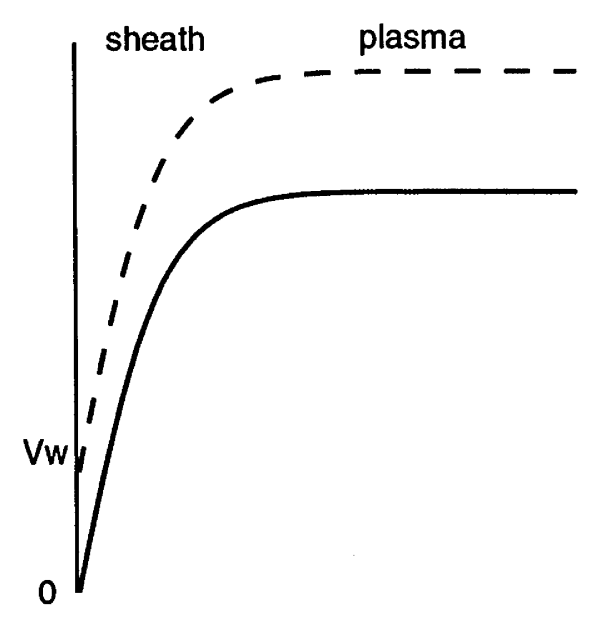

Figure 1.1: The potential profile of a wall sheath for a grounded wall (-) and a floating wall (--).

ion sound speed and equals $\sqrt{k T_{e} / m_{i}}$. During plasma breakdown, the wall may become charged to either a positive or negative potential $V_{w}$ if the wall is left floating (Charles, 2007). This can result in the potential profile moving up or down as shown by the dashed line in Figure 1.1.

The behaviour of sheaths at grounded walls, around objects immersed in plasma and in the plasma itself are particularly applicable to this thesis. Current-free double layers, discussed in the next section, can be considered as the combination of an 'ion sheath' and an 'electron sheath' that form within a plasma. Sheaths that form around diagnostics immersed in plasma can influence the results obtained and are discussed further in Chapter 3. Chapter 5 details the consequences of the sheath collapsing at a grounded wall and the implications for the use of RF antennas immersed in vacuum.

\subsection{Current-Free Double Layers}

An electric double layer is a narrow, localised region within a plasma, not connected to a wall, that sustains a large drop in the plasma potential (Block, 1978). This potential drop causes particles to accelerate producing an ion beam and/or an electron beam as shown in the schematic in Figure 1.2. In this schematic, the double layer is present between either two plasma sources separated by grids or apertures represented by the dashed line, or in an expanding plasma where no grids are present.

A double layer can be considered as two contiguous sheaths with opposite 


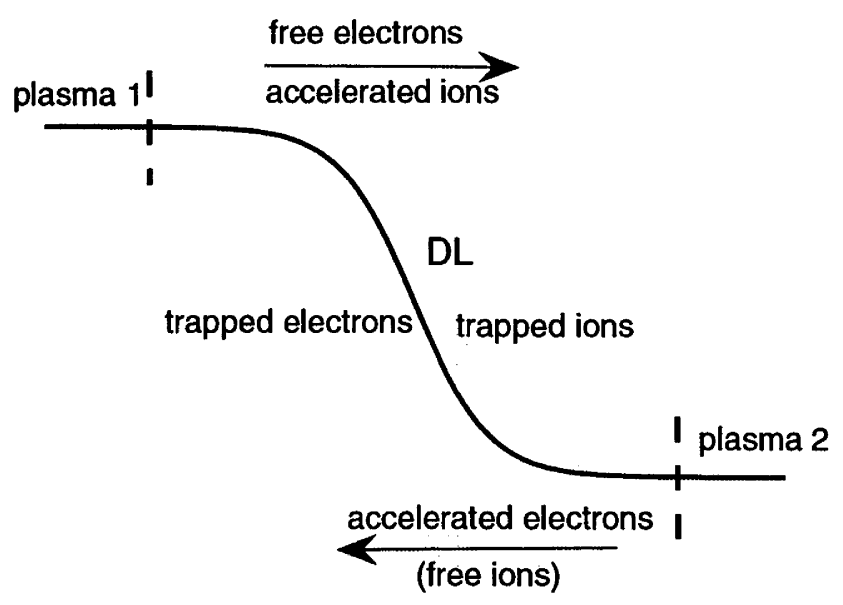

Figure 1.2: A schematic of the potential for a double layer in an expanding plasma. In most cases the free ion term is negligible as $k T_{i}<<k T_{e}<<e \phi_{D L}$

'signs', where quasi-neutrality breaks down within the double layer (Charles, 2007). One of the sheaths is composed of a layer of positive charge (an 'ion sheath') and the other of a layer of negative charge (an 'electron sheath'). For reference, the high potential side of the double layer is referred to as 'upstream' of the double layer and conversely, the low potential side is referred to as 'downstream'.

To be classified as a double layer, three generally accepted conditions must be satisfied (Meige, 2006):

1. The magnitude of the potential drop of the double layer, $\left|\Phi_{D L}\right|$, must be greater than $k T_{e} / e$ where $T_{e}$ is the electron temperature downstream of the double layer.

2. The electric field inside the double layer must be much stronger than outside the double layer and the global charge of the double layer must be zero.

3. At the position of the double layer, quasi-neutrality is locally violated.

Some researchers also suggest that to be considered a double layer, its thickness must be much smaller than the collisional mean free path of the plasma. However, this is not strictly necessary.

Double layers have been observed in a variety laboratory plasmas including in discharge tubes, double and triple plasma devices, $\mathrm{Q}$ machines and expanding plasmas. The details of these have been reviewed extensively elsewhere (Block, 
1978; Hershkowitz, 1985; Charles, 2007). Double layers have also been invoked to explain processes in the aurora, the solar corona and other astrophysical systems (Raadu, 1989; Borisov and Mall, 2002; Boswell et al., 2006; Ergun et al., 2009).

In most laboratory plasmas, a double layer is created by imposing a potential difference or by the application of a current to the system. The first measurements to suggest that double layers could be formed in the absence of large currents were made by Mozer et al. (1977), who made in situ measurements of DC electric fields at auroral latitudes and altitudes with the S3-3 satellite. Subsequent analytical work by Perkins and Sun (1981) showed that an abrupt potential drop surrounded by a quasi-neutral plasma with no electric field was possible and therefore current-free double layers could in fact exist. Current-free double layers have since been reported in triple plasma devices (Chan et al., 1983), Q machines (Hatakeyama et al., 1983) and, for a period of about $1 \mathrm{~ms}$, in a pulsed two-electron-population expanding plasma (Hairapetian and Stenzel, 1988).

\subsubsection{Recent Experimental Work}

Recently, Charles and Boswell (2003) have reported a stationary current-free double layer in a RF plasma that expands along a divergent magnetic field. The experimental device, known as Chi Kung, is shown in Figure 1.3a and consists of a helicon plasma source attached to a larger diameter vacuum chamber. Chi Kung was originally designed to investigate plasma processing techniques for materials science applications. Figures $1.3 \mathrm{~b}$ and $1.3 \mathrm{c}$ show the axial plasma potential and plasma density profiles measured when operating with argon at $0.2 \mathrm{mTorr}$ with $250 \mathrm{~W}$ of RF power from Charles and Boswell (2003). The discontinuity associated with the double layer can be clearly seen, particularly in the plasma potential profile. The double layer was only observed at pressures below $2 \mathrm{mTorr}$ in argon and as the pressure was decreased the potential drop of the double layer increased. The double layer has been shown to form during plasma breakdown and is stable and stationary afterwards (Charles and Boswell, 2004b)

Downstream of the double layer, a supersonic ion beam was detected with an energy analyser. In argon at 0.2 mTorr, the ion beam was found to have a constant velocity, $v_{b}$, across the radius of the source tube of about $15 \mathrm{kms}^{-1}$ and a beam divergence of less than $10^{\circ}$ (Charles and Boswell, 2004a; Charles et al., 2006). Subsequent detailed experiments have shown that the average ion beam divergence is $9^{\circ}$ and that $98 \%$ of the momentum is imparted along the centreline of the source tube (Cox et al., 2008a). Ion beams have also be measured in Chi Kung when using a variety of different gases including hydrogen, xenon, methane, ammonia, 


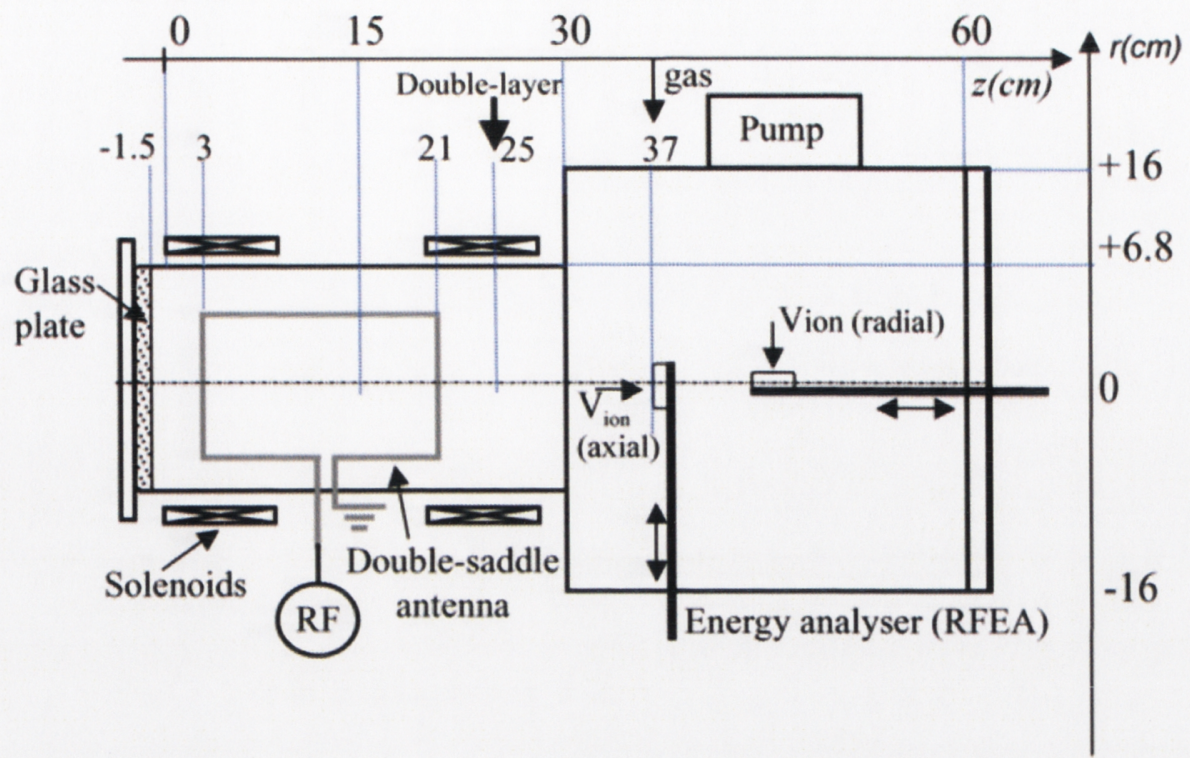

(a)

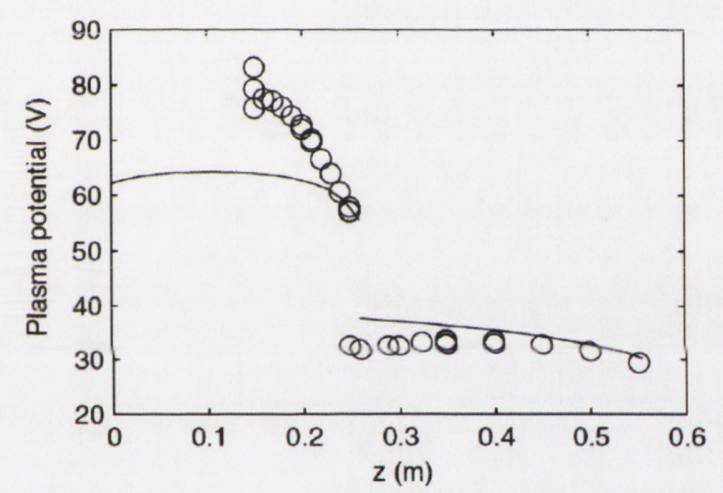

(b)

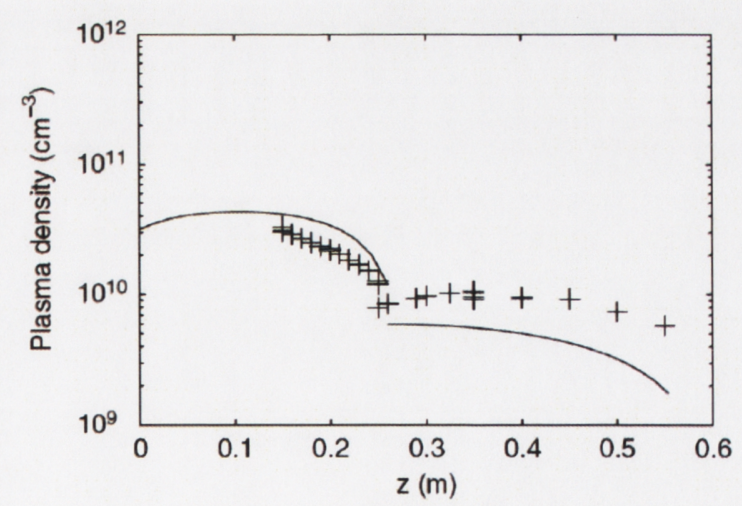

(c)

Figure 1.3: A schematic of the Chi Kung experiment (a) and the axial plasma potential profile (b) and axial plasma density profile (c) when operating with argon at 0.2 mTorr with $250 \mathrm{~W}$ of RF power revealing the discontinuities associated with the double layer (Charles and Boswell, 2003). The solid lines in (b) and (c) show the theoretical results determined from a model developed by Lieberman and Charles (2006). 
nitrogen, nitrous oxide, oxygen and carbon dioxide (Charles, 2004; Charles et al., 2006, 2008b; Charles, 2009; Charles et al., 2009).

Around the same time, Cohen et al. (2003) also reported ion acceleration in plasmas emerging from a helicon-heated magnetic-mirror device known as MNX. Since then, current-free double layers and the associated ion beams have been reported in a variety of low pressure expanding magnetised plasmas. These include other helicon reactors, devices with larger and smaller diameter sources and downstream vacuum chambers, inductively coupled plasmas and devices with a variety of magnetic field configurations. Electronegative double layers have also been measured recently by Plihon et al. (2005) in mixtures of $\mathrm{Ar} / \mathrm{SF}_{6}$ in the absence of a magnetic field, but the potential drop of the double layer is less than the electropositive case. Table 1.3 summarises these devices and the typical operating conditions where electropositive ion beams and/or double layers are found.

\subsubsection{Theoretical and Simulation Work}

Several efforts have attempted, both analytically and theoretically, to model the formation of the current-free double layer in these experimental devices. One model, developed by Lieberman et al. (2006), assumes five species of particles: trapped and free electrons, thermal and accelerated ions and a population of electrons accelerated from downstream to upstream. However, this does not include the presence of the magnetic field and to date no evidence of an electron beam upstream of a current-free double layer has been found (Takahashi et al., 2007). Chen (2006) has derived an analytical model of a plasma expanding in a diverging magnetic field based on classical sheath theory and Fruchtman (2006) has theoretically investigated the momentum imparted by a current-free double layer to a collisionless plasma. Several other models have also been recently developed (Goswami et al., 2008; Ahedo and Martinez-Sanchez, 2008).

Numerical particle in cell (PIC) simulations have been developed and the results were in good agreement with the current-free double layers and ion beams observed experimentally in the Chi Kung and HELIX devices (Meige et al., 2005a,b; Meige, 2006; Sun et al., 2005b; Keese et al., 2005). Similar PIC simulations have been used to model current-free double layers in electronegative plasmas (Meige et al., 2007). The ion acceleration observed in the MNX experiment has recently been simulated with a three-dimensional PIC model (Sefkow and Cohen, 2009). 


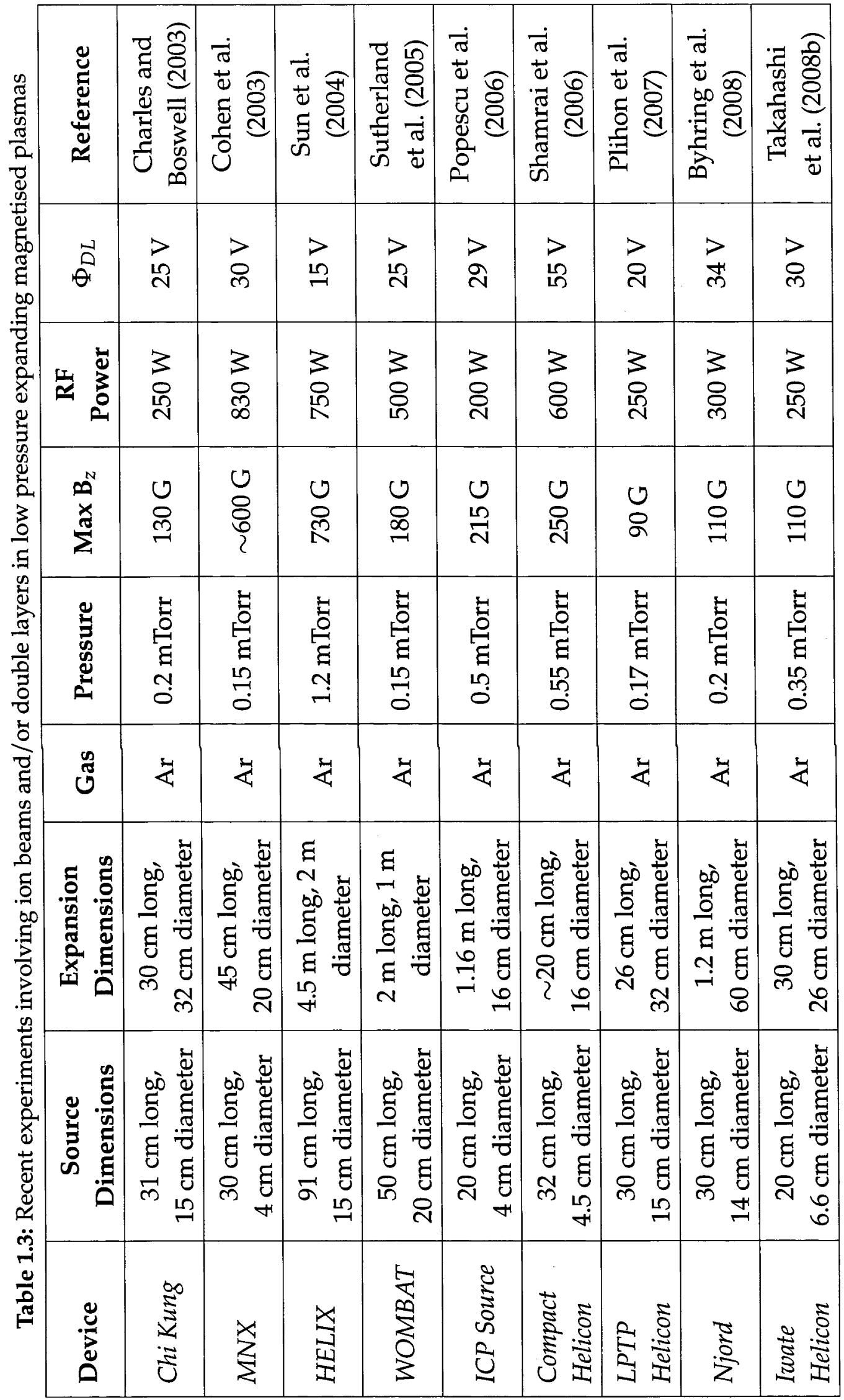




\subsection{The Helicon Double Layer Thruster Concept}

Both Charles and Boswell (2003) and Cohen et al. (2003) realised that their discovery of energetic ions emanating from low-pressure helicon sources had applications to spacecraft propulsion. The Helicon Double Layer Thruster (HDLT) concept, first conceived and patented by Charles and Boswell (2002), is a magnetoplasma thruster based on the Chi Kung experiment. The HDLT concept uses a helicon source, that is driven by a double-saddle field antenna, to create an expanding magnetised plasma containing a current-free double layer. It is proposed that thrust is produced when a beam of ions is ejected by the double layer at supersonic velocities at the thruster exit.

\subsubsection{Advantages and Applications}

RF plasma sources, and in particular the helicon source, are an attractive option for future spacecraft electric propulsion systems. Compared to capacitive and inductive plasma sources operating at similar RF powers, helicon sources yield higher plasma densities, e.g. $n \backsim 1 \times 10^{19} \mathrm{~m}^{-3}$ for a $1 \mathrm{~kW}$ helicon source using argon versus $\sim 1 \times 10^{16} \mathrm{~m}^{-3}$ and $\sim 1 \times 10^{17} \mathrm{~m}^{-3}$ for capacitive and inductive sources, respectively (Scime et al., 2008). From Equation 1.7, a higher ionisation density means a higher mass flow rate of ionised propellant, which results in higher thrust and greater propellant utilisation efficiency.

Helicon sources have been proposed for generating ions for several different spacecraft electric propulsion systems, including VASIMR and the high power helicon (HPH) thruster (Ziemba et al., 2006). However, compared to the HDLT concept, these systems require very high input powers (in the order of tens of kilowatts) and high magnetic fields (>500 G). A helicon source has also been recently proposed as the ionisation stage for electric propulsion using ion-ion plasmas that accelerate both positive and negative ions (Aanesland et al., 2009).

The ion beam produced by the HDLT has a very low beam divergence (less than $10^{\circ}$ for argon and $6^{\circ}$ for xenon) and a constant velocity across the thruster radius (Charles et al., 2006; Cox et al., 2008a). Magnetic steering of the ion beam has been demonstrated (Charles et al., 2008a) and this innovation would remove the need for the complex mechanical thrust vectoring systems (gimbals) currently used on spacecraft equipped with electric propulsion. Exhaust velocities of up to $15 \mathrm{kms}^{-1}$ have been measured in argon, $10 \mathrm{kms}^{-1}$ in xenon and even $70 \mathrm{kms}^{-1}$ in hydrogen (Charles and Boswell, 2004a; Charles, 2005b, 2009). These properties 
lead to a moderately high specific impulse of between 1000 and 1500 seconds for xenon and argon respectively, and a high thrust-vector efficiency (Gesto et al., 2006).

The HDLT is scalable in both size and power, with various experiments worldwide demonstrating ion beam formation in helicon sources of various diameters and operating at various levels of RF power (Sutherland et al., 2005; Shamrai et al., 2006). It can also operate in a pulsed or continuous mode (Charles and Boswell, 2004b). The HDLT has no electrodes, accelerating grids or neutraliser, which limit the operating life of other electric propulsion systems. No accelerating grids are required as the double layer acts as a 'virtual' grid and is selfsustained by the plasma. A neutraliser is not required as sufficient free electrons can overcome the potential of the double layer and neutralise the ion beam downstream (Takahashi et al., 2007).

Erosion of the Pyrex source tube is possible, but this depends upon the impact energy of the ions onto the source tube walls. This energy is a function of the wall sheath potential, which has been measured and modelled (Lieberman et al., 2006) as typically less than $100 \mathrm{eV}$. The $\mathrm{SiO}_{2}$ etch rate for argon ions, for example, is near-zero below $200 \mathrm{eV}$ (Harper et al., 1981) and therefore such erosion will be minimal. Since the HDLT has no moving parts and no failure modes associated with electrodes, grids or neutralisers, the HDLT is an attractive option for large spacecraft requiring high $\Delta v$ and long duration missions. These missions could include interplanetary probes with large science payloads, cargo missions to Mars and the Moon, and long duration satellite station-keeping in Earth orbit.

Furthermore, since the double layer and ion beam have been reproduced in a variety of different gases, as discussed in Section 1.5.1, a variety of propellant options are available. Charles et al. (2008b) have proposed that the HDLT could make use of residual gaseous hydrazine propellant left aboard spacecraft that use standard chemical thrusters. Using the last drops of propellant, an additional $\Delta v$ could be used to de-orbit a satellite or move it to a graveyard orbit. Oxygen, hydrogen and carbon dioxide can also be used as propellants for the HDLT, which may enable the use of waste products produced by crewed missions and aboard the International Space Station. Atmospheric carbon dioxide from Venus or Mars or ground reservoirs of water on the Moon, Mars and Mars' moon Phobos (West and Lee, 2007) could also be a source of propellant. 


\subsubsection{Testing Campaign at ESTEC}

In 2005, under the auspices of an Australian government Innovation Access Grant, the first HDLT prototype was constructed and tested in the CORONA space simulation chamber at the European Space Agency (ESA) development centre ESTEC. The CORONA space simulation chamber consisted of a $1.4 \mathrm{~m}$ long, $1.1 \mathrm{~m}$ diameter hatch in which the HDLT prototype was mounted. This was attached to the $5 \mathrm{~m}$ long, $2 \mathrm{~m}$ diameter CORONA chamber that was equipped with cryogenic pumps and had an effective pumping speed for xenon of 12,000 L.s ${ }^{-1}$. The hatch had a pumping speed of 7,000 L.s ${ }^{-1}$.

The HDLT prototype was mounted on a two-axis thrust balance developed by Alta S.p.A (Banetta et al., 2004). Unfortunately, the thrust balance was still in its commissioning phase and was not operating correctly. In addition, the testing campaign was very brief ( $\sim 5$ weeks) and the space simulation chamber was fitted with limited diagnostics so little could be determined about the plasma produced or if an ion beam was formed. However, a new high density 'blue' mode was discovered when operating in xenon (Charles et al., 2008). This mode is discussed further in Chapter 6.

\subsubsection{Unresolved Questions}

All previous experiments where double layers and the associated ion beam have been observed consisted of a plasma source tube attached to a larger diameter vacuum chamber. These devices are based on systems used for materials processing (ChiKung and other helicon reactors) or are used for studies of fundamental plasma and astrophysical phenomena (WOMBAT, MNX and HELIX). If current-free double layers are to be used in electric propulsion applications, it is imperative that such a system is tested in a configuration where the plasma source (and the whole thruster) is immersed inside a space simulation vacuum chamber. Such a configuration more accurately reflects the proposed application and will help determine if an ion beam is formed by the HDLT prototype. These tests will also help determine if the presence of the double layer and hence the ion beam depend upon the physical geometry of the source tube and that of the expansion region. In other words, is a double layer formed when the region downstream of the source is infinite as it would be in the case of a spacecraft thruster. It is also unknown if the double layer is dependent upon the interaction of the downstream plasma and to what extent the downstream plasma influences the double layer and ion beam properties. 
Inferences about the thrust levels expected from the HDLT concept have been made based upon the measured plasma parameters and the properties of the ion beam. Estimates have ranged from a few $\mathrm{mN}$ to tens of $\mathrm{mN}$ depending upon the plasma conditions and the assumptions made (Charles and Boswell, 2003, 2008; Charles, 2009). However, to date, no direct measurements have been made of the thrust, if any, that is produced. The influence of the operating pressure, RF power, magnetic field strength and configuration, source geometry and propellant choice upon the thrust levels also awaits characterisation.

Further experimental work is required with the HDLT concept in a configuration that more accurately reflects the proposed application; that is, with the whole thruster immersed in vacuum. By characterising the various operating modes and the influence of the different operating parameters on the plasma and ion beam produced, the HDLT concept can be evaluated for future spacecraft missions and progress towards a future flight opportunity will be advanced. This forms the primary motivation for this thesis.

\subsection{Thesis Scope}

This thesis presents results from the first comprehensive tests of the HDLT prototype immersed in a space simulation chamber. In Chapter 1, the principles behind spacecraft propulsion have been reviewed and the rationale for electric propulsion has been presented. Background information on the use of plasmas for spacecraft propulsion has been outlined and current-free double layers have been reviewed including recent experimental and theoretical work. Finally, the HDLT concept has been presented along with the some of the advantages and applications of this new propulsion system. The details of some unresolved questions regarding the HDLT concept are also discussed. These provide the motivation for this thesis.

Chapter 2 outlines the experimental apparatus and diagnostics used in this thesis, including a description of the HDLT prototype, the space simulation vacuum chamber, the Langmuir probe and the Retarding Field Energy Analyser (RFEA). In Chapter 3, the principles behind thrust measurement and the challenges associated with using conventional techniques with the HDLT prototype are discussed. A new momentum flux measuring instrument (MFMI) developed especially for use with the HDLT prototype is presented and the design, construction, calibration and operation of this instrument is detailed.

The first experimental evidence of an ion beam formed by a current-free double 
layer with the HDLT prototype immersed in vacuum is presented in Chapter 4. Using the Langmuir Probe and the RFEA, the ion beam and plasma are characterised axially and radially when operating at low powers with argon. The influence of the various operating parameters, including pressure and magnetic field are characterised and the results are compared with previous current-free double layer experiments. Measurements in the exhaust of the HDLT prototype with the MFMI are presented and measurements of the ion beam created at low power in xenon are also outlined. Although not a focus of this thesis, some conclusions are also drawn regarding plasma detachment based on the experimental results obtained.

Chapter 5 outlines the challenges associated with operating the RF antenna of the HDLT prototype immersed in vacuum, including parasitic plasma formation and microarcing. Several solutions are proposed and the installation of a blocking capacitor that electrically floats the antenna is implemented. This approach enables operation of the HDLT prototype in vacuum at higher RF powers than previously possible. Chapter 6 presents results when operating the HDLT prototype at higher RF powers with xenon. The Langmuir probe, RFEA and MFMI are used to conduct the first detailed characterisation of the high density 'blue' mode in xenon, which was previously discovered during the ESTEC testing campaign. Inferences are made about the plasma properties in this mode by investigating the changes in the matching box circuit and a preliminary spectroscopic study of the plasma in the HDLT source and exhaust is undertaken. Finally, Chapter 7 summarises the conclusions of this thesis and presents several avenues for future research. 


\section{Experimental Apparatus and Diagnostics}

\subsection{The HDLT Prototype}

The Helicon Double Layer Thruster (HDLT) prototype was constructed prior to the commencement of this thesis, as part of an Australian government Innovation Access Grant, by the Space Plasma, Power and Propulsion Group at the Australian National University in conjunction with Auspace Limited and the Cooperative Research Centre for Satellite Systems (CRCSS), which is managed by the Commonwealth Scientific and Industrial Research Organisation (CSIRO). It was developed for the testing campaign at ESTEC described earlier in Section 1.6.2. The design of the HDLT prototype is based on the helicon plasma source Chi Kung originally developed for materials processing research and in which a current-free double layer was found experimentally (Charles and Boswell, 2003).

The HDLT prototype, as shown in Figures 2.1 and $2.2^{\dagger}$, consists of a Pyrex source tube, with a closed end of Pyrex, that has a $5 \mathrm{~mm}$ wall thickness. The source tube has an outer diameter of $15 \mathrm{~cm}$, an inner diameter of $14 \mathrm{~cm}$ and has a length and hence insulating plasma cavity of $29 \mathrm{~cm}$. The source tube is mounted inside the HDLT structure, which is made of sand-blasted aluminum. The HDLT structure consists of three rectangular panels for thermal radiation, mounted perpendicular to the axis of the source tube, and two solenoids, the axes of which are mounted parallel to the axis of the source tube. The origin of the $\mathrm{z}$-axis in this experiment is referenced to the rectangular panel at exhaust end of the HDLT structure ( $\mathrm{A}$ in Figure 2.2) and is defined as $\mathrm{z}=0 \mathrm{~cm}$. The source tube of the

\footnotetext{
${ }^{\dagger}$ These views were extracted from a Solidworks model developed by Auspace Limited.
} 


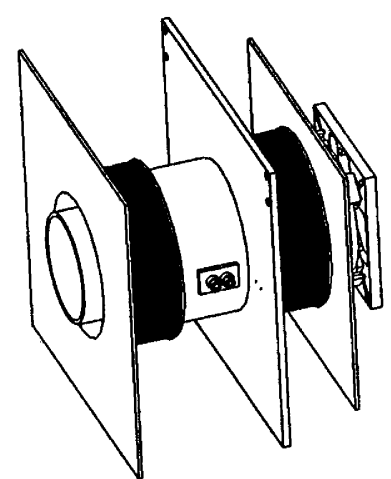

(a) Isometric view

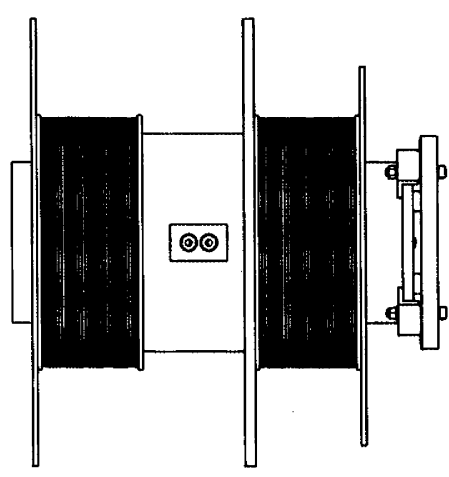

(b) Side view

Figure 2.1: The Helicon Double Layer Thruster prototype.

HDLT prototype can be moved axially with respect to the HDLT structure and the solenoids. For the vast majority of experiments in this work, the source tube is positioned so it extends $3 \mathrm{~cm}$ beyond the HDLT structure (i.e. the end of the source tube is at $\mathrm{z}=3 \mathrm{~cm}$ ).

The propellant inlet consists of a piece of insulating Macor machinable ceramic that is inserted into a hole in the closed end of the source tube (and plasma cavity) and is affixed with a vacuum compatible adhesive. The source tube is attached to an aluminum interface plate $((\mathrm{F})$ in Figure 2.2). The interface plate allows the source tube to be mounted to a thrust balance so that the source can move independently of the HDLT structure. This configuration was used during the tests of the HDLT prototype at ESTEC described in Section 1.6.2. The interface plate can also be used to affix the source tube in the one location and attach it directly to HDLT structure. In this thesis, the HDLT source tube is attached directly to the HDLT structure via the interface plate.

The solenoids produce a divergent magnetic field and are wound from $1.4 \mathrm{~mm}$ diameter polyamide-imide coated copper wire suitable for use in vacuum environments. Each solenoid is singly wound and consists of 774 turns or $486 \mathrm{~m}$ of wire. To reduce the thermal loading on the HDLT and to avoid overheating and melting of the solenoid wire, the current applied to each solenoid was limited to $6 \mathrm{~A}$ and was typically operated at $3 \mathrm{~A}$ on each coil since this was the reference condition used during previous experiments with the Chi Kung experiment. When operating at $3 \mathrm{~A}$, the axial maximum produced is $138 \mathrm{G}$ at $8 \mathrm{~cm}$ upstream from the exit of the source tube which decreases to a few Gauss downstream. The magnetic field topography is discussed further in Section 2.4. 


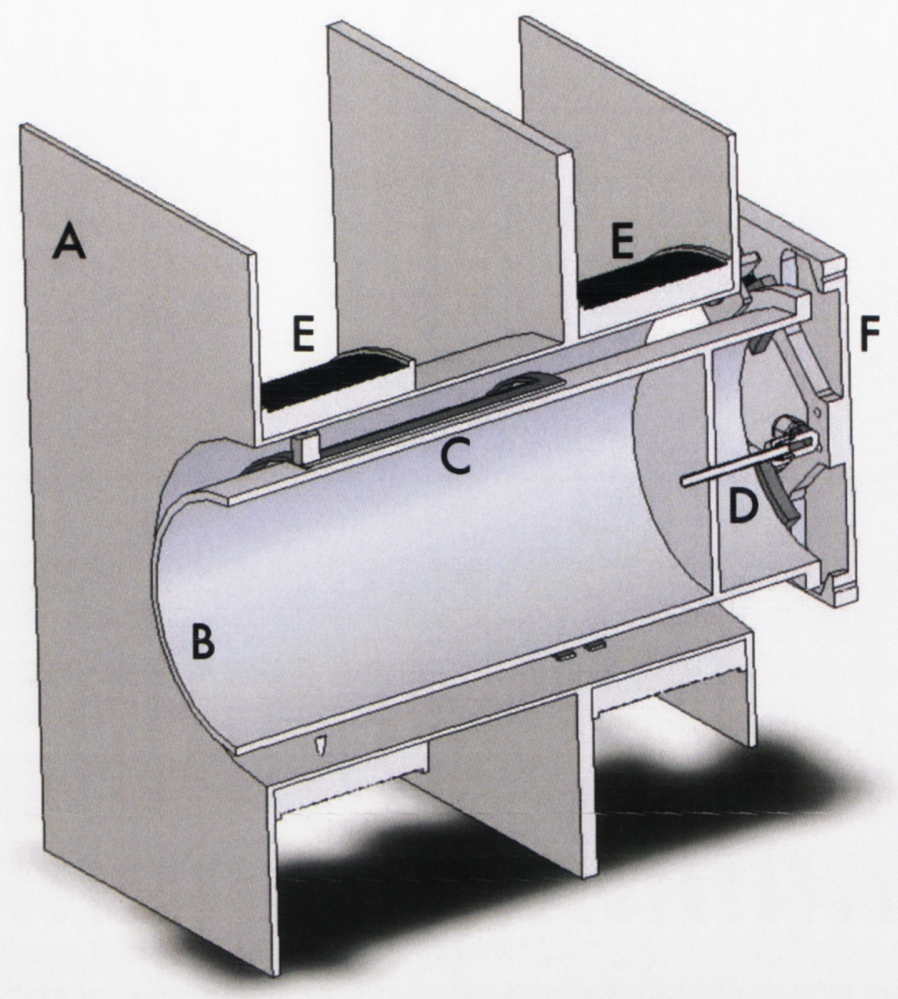

Figure 2.2: A cut away view of the HDLT prototype showing the HDLT structure (A), source tube (B), antenna (C), propellant inlet (D), solenoids (E) and interface plate (F).

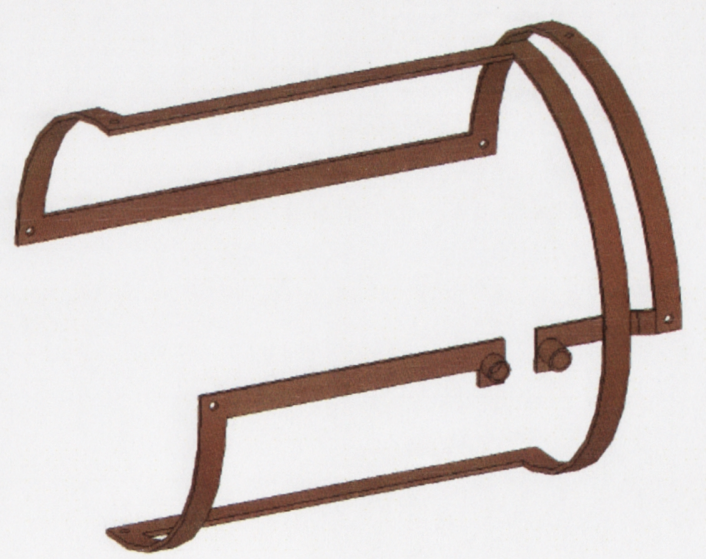

Figure 2.3: The double-saddle field antenna used in the HDLT prototype. 
A double-saddle field antenna constructed from copper with a $25 \mu \mathrm{m}$ silver plating and $18 \mathrm{~cm}$ long surrounds the source tube. It is based on that invented by Boswell (1970) and is shown in Figure 2.3. The antenna is attached to and electrically isolated from the inside of the grounded HDLT structure by Macor insulating standoffs. The antenna is a few millimetres from the source tube to limit thermal effects. The antenna is not in contact with the source tube, allowing for the source tube to be moved relative to the antenna and HDLT structure by adjusting the interface plate if required. The HDLT structure also includes feedthroughs, insulated with Macor pieces, for connecting the radio frequency network, described in Section 2.3, to the antenna.

\subsection{Space Simulation Chamber}

The HDLT prototype is installed, as shown in Figure 2.4 and 2.5a, inside a space simulation vacuum chamber $1 \mathrm{~m}$ in diameter and $1.4 \mathrm{~m}$ long. The chamber is manufactured from non-magnetic stainless steel that is resistant to deformation caused by thermal cycles, high vacuum and outgassing to simulate the vacuum conditions of low Earth orbit, where the pressures are typically less than $10^{-6}$ Torr (Tribble, 2003). The HDLT prototype hangs in position from two rails mounted on the roof of the space simulation chamber. Along with two rails on the floor of the chamber, these also provide useful attachment points inside the space simulation chamber.

The apparatus has been designed so that a single port, $25 \mathrm{~cm}$ in diameter, on the side of the space simulation chamber can provide feedthroughs for the propellant line, power for the solenoids and radio-frequency (RF) power for the antenna (Figure 2.5b). The propellant, typically argon or xenon, is injected into the source tube via nylon tubing attached to a short length of Macor tube that feeds into the closed end of the Pyrex source tube. The propellant flow rate is regulated by a mass flow controller mounted outside the vacuum chamber. The flow controller used is a MKS Type 2160B Mass Flow Controller that has an accuracy of $\pm 1 \%$. A $10 \mathrm{sccm}$ or $100 \mathrm{sccm}$ (for $\mathrm{N}_{2}$ ) mass flow controller is used depending upon the flow rate required. SCCM denotes standard cubic centimetres per minute at standard temperature and pressure and all flow rates quoted in this thesis are the real values, following calibration, for the relevant gas species used.

A TTi CPX 400 Dual 42 V 20 A Power Flex Power Supply is used to power the solenoids and is connected to an ISI 9423058 6-pin vacuum power feedthrough. This feedthrough is rated to $5000 \mathrm{~V}$ at $25 \mathrm{~A}$ and attaches to one of the ports on the 


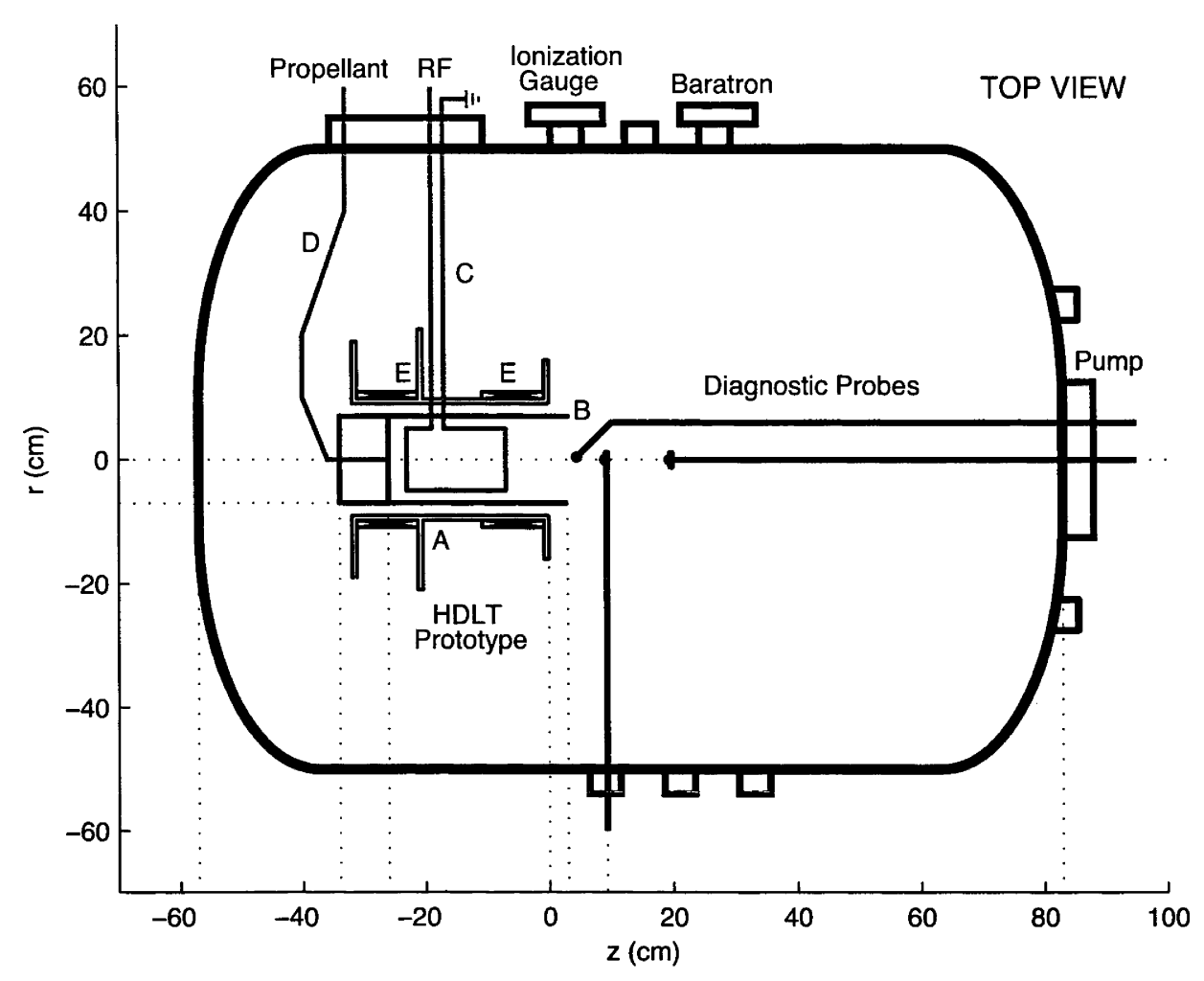

Figure 2.4: The HDLT prototype integrated into the space simulation chamber. The HDLT structure (A), source tube (B), antenna (C), propellant line (D) and solenoids (E) are shown along with the diagnostic probes.

side of the vacuum chamber. It is connected to leads inside the vacuum chamber that run to two ceramic terminal blocks connected to solenoids and affixed to the HDLT structure. Other ports on the sides and ends of the space simulation chamber provide access for axial and radial diagnostic probes and window ports allow the operation of the HDLT prototype to be viewed from various angles. The window ports also allow access for the laser beam that is used to measure the displacement of the Momentum Flux Measuring Instrument, which is described in Chapter 3.

The vacuum chamber has a pumping system consisting of a Leybold Turbovac $600 \mathrm{C}$ turbomolecular pump, that is rated for $600 \mathrm{~L} . \mathrm{s}^{-1}$ for $\mathrm{N}_{2}$, in series with an Alcatel Adixen PASCAL 2021 SD rotary pump. A MDC KAV-150 shut-off valve is installed between the turbomolecular pump and the rotary pump to prevent unwanted back-flow of rotary pump oil into the vacuum chamber via the turbomolecular pump. The pumping system maintains a base pressure less than 


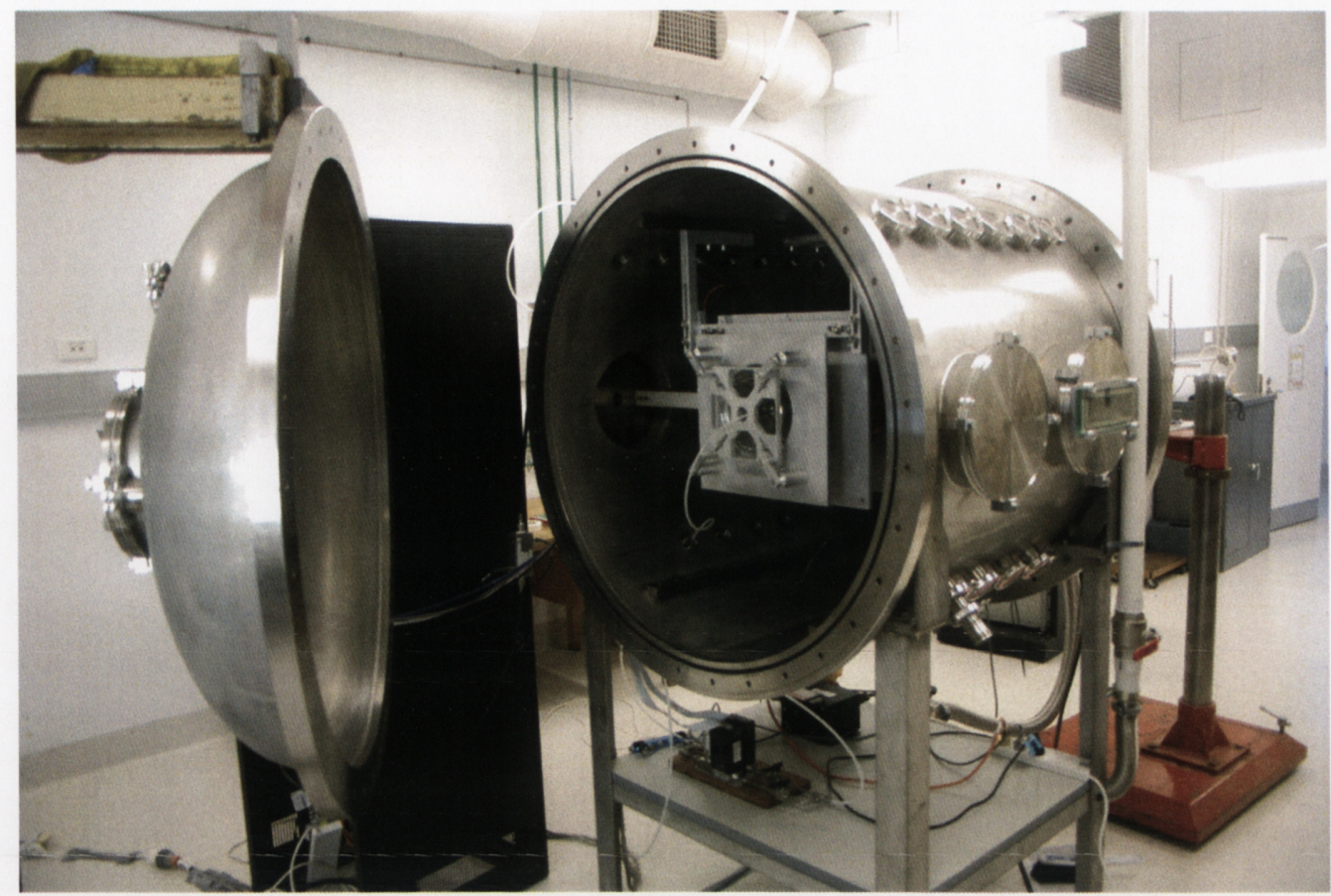

(a)

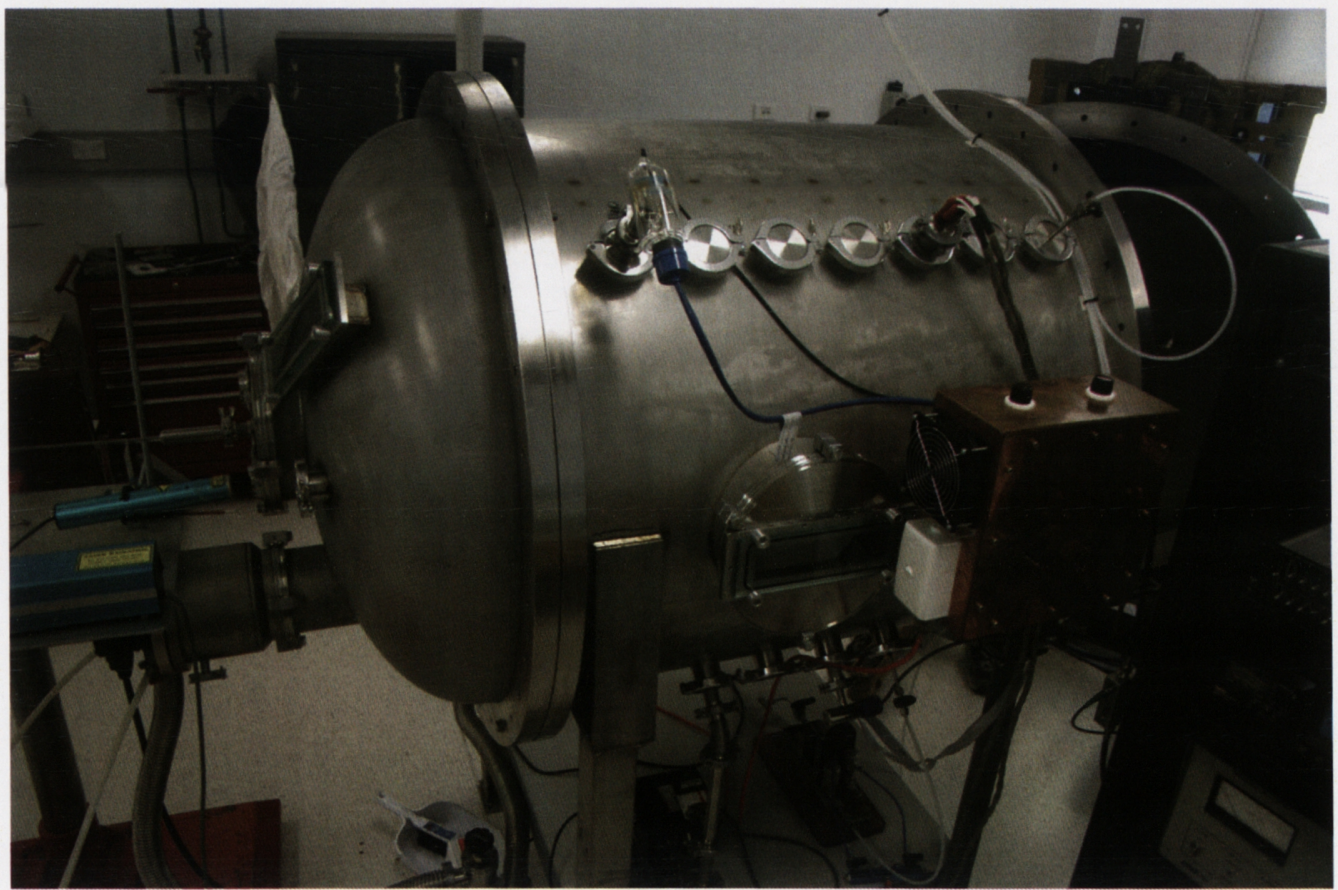

(b)

Figure 2.5: Image of (a) the HDLT prototype installed inside the space simulation chamber and (b) the side view of the space simulation chamber showing the matching box and access ports. 
$1.0 \times 10^{-7}$ Torr and the effective pumping speed measured for argon is approximately $330 \mathrm{~L} . \mathrm{s}^{-1}$ and for xenon is approximately $210 \mathrm{~L} . \mathrm{s}^{-1}$. At such pressures the thermal environment of outer space, that is beyond the Earth's atmosphere, can be simulated, as the thermal conduction of gases is small relative to the radiant heat transfer. The gas flow inside the space simulation chamber is also in the free molecular-flow regime, where each gas acts independently of all others present (Holkeboer et al., 1967). The chamber pressure is measured using a Granville-Phillips Convectron Gauge, a MKS 220CA Baratron Gauge and a Granville-Phillips Series 274 Ionization Gauge Tube, which are located on the vacuum chamber as shown in Figure 2.4. The stated base pressure is measured with the Ionization Gauge Tube and corrected for argon, whose gas correction factor compared to nitrogen is 1.29. The gas correction factor for xenon is 2.87 . All other pressure measurements in this work are made with the Baratron Gauge, which is gas species independent, so no correction for argon or xenon is required.

As shown in Figure 2.4, the system is divided into the downstream region of the space simulation vacuum chamber (positive numbers) and the source region of the HDLT prototype (negative numbers). The exit of the HDLT source tube is principally located at $\mathrm{z}=3 \mathrm{~cm}$ and the back wall of the chamber is located at $\mathrm{z}=83 \mathrm{~cm}$. Radially, the centreline of the HDLT source tube and the space simulation chamber are at $\mathrm{r}=0 \mathrm{~cm}$ and the wall of the chamber is at $\mathrm{r}=50 \mathrm{~cm}$.

\subsection{Radio Frequency Matching Network}

A $13.56 \mathrm{MHz}$ radio frequency ( $\mathrm{RF}$ ) matching network/generator on the outside of the vacuum chamber is connected to the antenna of the HDLT by two $6 \mathrm{~mm}$ diameter, $\sim 40 \mathrm{~cm}$ long copper rods. At one end, the copper rods attach to the antenna and are electrically isolated from the aluminum HDLT structure by Macor standoffs. The copper rods extend radially towards a port on the side of the vacuum chamber and are enclosed in a grounded copper shield to form a Faraday cage for shielding. The ends of the copper rods at the port on the vacuum chamber wall are connected to an ISI 951200 vacuum RF feedthrough, which forms part of the RF matching box. The vacuum RF feedthrough is rated for up to $8000 \mathrm{~V}$ and $10 \mathrm{~kW}$ at $450 \mathrm{kHz}$.

The matching network is a custom built $L$ impedance matching network that uses two tunable vacuum capacitors housed in a matching box constructed from copper sheet and fan cooled. Figure 2.6 shows a schematic of the matching network and the antenna assembly described above. The $C_{1}$ vacuum capacitor is in series 


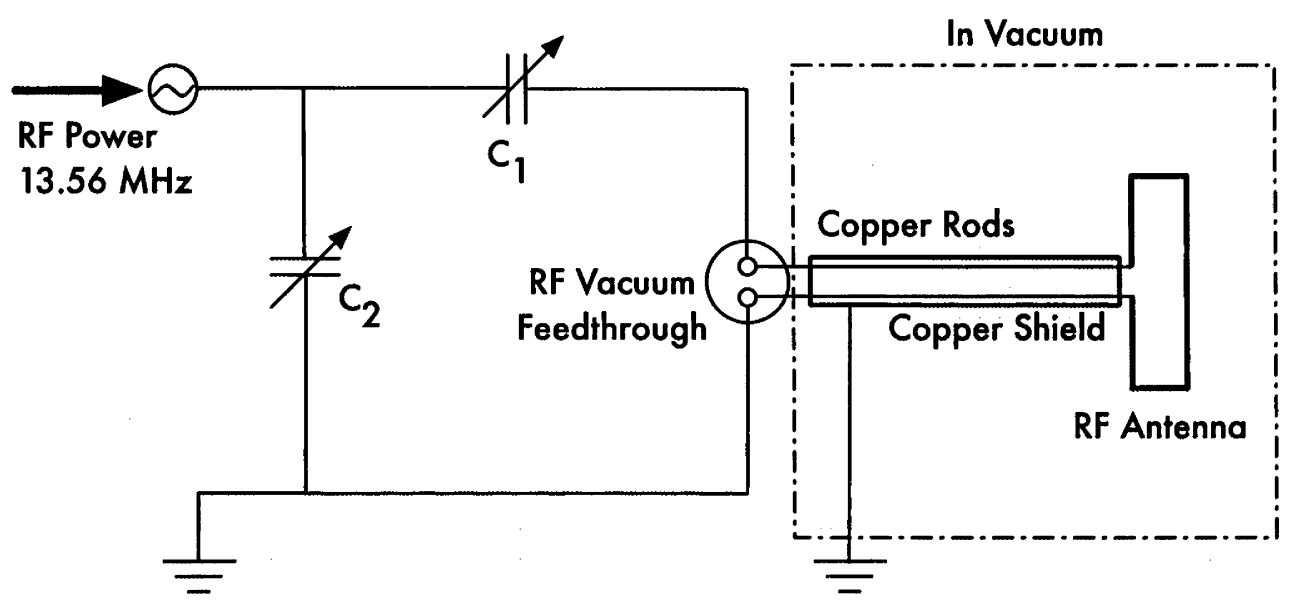

Figure 2.6: A schematic of the $L$ impedance matching network including the antenna, copper rods and grounded copper shield that are located in vacuum.

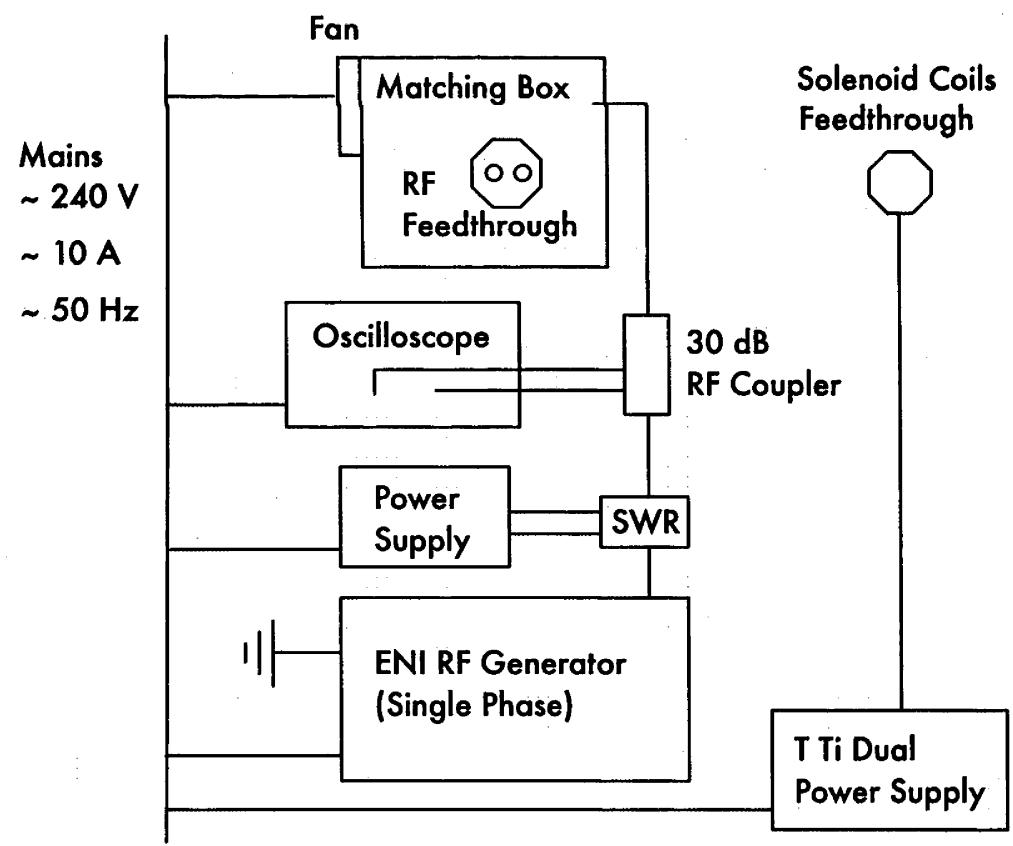

Figure 2.7: The electrical system for the HDLT prototype that provides the RF power and the magnetic field. 
and is a SCV-155M MEIVAC SCV tunable vacuum capacitor rated for up to $15 \mathrm{kV}$ at $500 \mathrm{pF} . \mathrm{C}_{2}$ is in parallel and is a SCV-520M MEIVAC SCV tunable vacuum capacitor rated for up to $5 \mathrm{kV}$ at $2000 \mathrm{pF}$. The inductance of the antenna, including the two copper rods and the vacuum feedthrough, was measured using a Bonton Electronics Corp. Capacitance-Inductance Meter and found to be $9.5 \mu \mathrm{H}$.

The matching box is used to tune the plasma using $C_{1}$ and $C_{2}$ and is connected to a Revex W502 S.W.R. (Standing Wave Ratio) \& Power Meter, which is used to measure the forward and reflected power. The matching box is also connected to a $30 \mathrm{~dB}$ radio frequency coupler that is attached to a Phillips PM3302 oscilloscope which also enables the forward and reflected power to be monitored. The RF power (13.56 MHz) is provided by an ENI Power Systems ACG-10 single phase radio frequency generator with a maximum output of $1200 \mathrm{~W} .100 \mathrm{~W}$ of forward power is typically maintained to reduce the thermal loading on the HDLT prototype. The operation of the HDLT prototype at higher RF powers is discussed in detail in Chapter 6, along with the challenges faced and the modifications required to enable it (Chapter 5). Figure 2.7 shows a schematic of the electrical system for the RF power supply and the solenoid coils.

\subsection{Magnetic Field Topography}

The magnetic field configuration plays a critical role in the operation of the HDLT prototype, as the formation of the double layer and subsequent ion beam are associated with the presence of a diverging magnetic field. Figure 2.8a shows the calculated magnetic field topography when $3 \mathrm{~A}$ is applied to each solenoid coil. The diverging nature of the magnetic field can be clearly seen. The centre of the downstream, or exhaust, solenoid is located at $\mathrm{z}=-5.7 \mathrm{~cm}$ and the centre of the upstream solenoid is positioned at $\mathrm{z}=-26.2 \mathrm{~cm}$.

Figure $2.8 \mathrm{~b}$ shows the calculated and measured axial DC magnetic field component, $B_{z}$, as a function of axial position, when a current of $3 \mathrm{~A}$ is applied to each solenoid coil. $B_{z}$ was measured using a 3-axis Bell 640 Hall effect Gaussmeter that has an accuracy of $\pm 0.65 \%$. The axial position accuracy of the Gaussmeter is $\pm 5 \mathrm{~mm}$. To highlight the location of the magnetic field relative to the HDLT, a schematic of the HDLT is also shown in Figure 2.8b. The maximum of the magnetic field is at $z=-5 \mathrm{~cm}$ and the maximum magnetic field gradient is at $\mathrm{z}=2 \mathrm{~cm}$. The measured values for $B_{z}$ are in good agreement with that calculated for these conditions. 


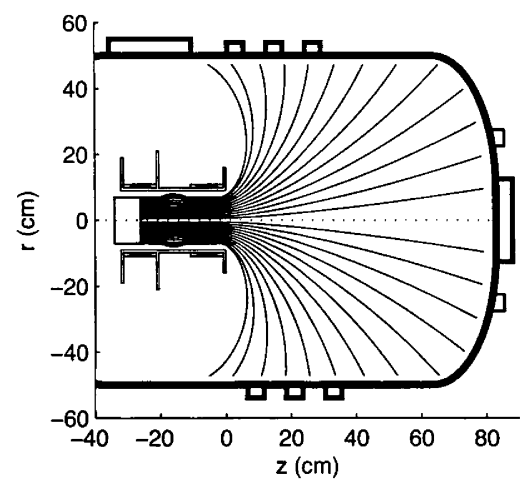

(a)

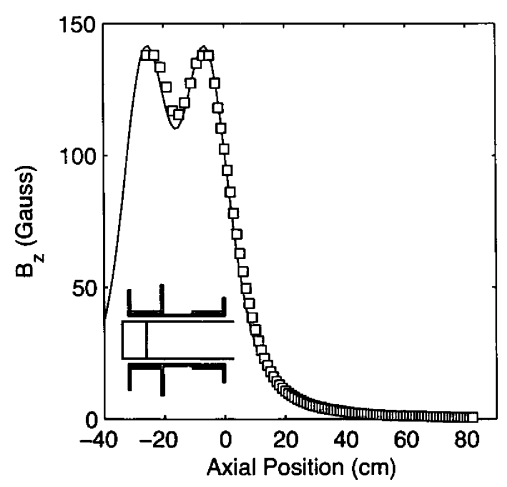

(b)

Figure 2.8: The calculated magnetic field lines (a) and the calculated (-) and measured ( $\square) B_{z}$ component of the DC magnetic field along the axis (b) from the inside of the HDLT source to the end of the space simulation chamber. The schematic of the HDLT prototype and the space simulation chamber are also shown. The antenna and other details have been removed for clarity.

\subsection{Langmuir Probe}

\subsubsection{Principles of Operation}

A Langmuir probe is one of the simplest and cheapest plasma diagnostics available and was first invented by Langmuir in 1926, after whom it is named (MottSmith and Langmuir, 1926). By placing a small conducting surface, typically a wire or disk, in a plasma and applying a bias voltage a current is drawn from the plasma. This current depends on the recent plasma conditions at the location of the probe. A large body of work on the physics of Langmuir probes exists and many reviews (Braithwaite and Franklin, 2009; Godyak et al., 2002; Hershkowitz, 1989; Chen, 1965) have been written so the details will not be reviewed here. Rather, an overview of the measurement techniques used in this thesis will be presented.

A current-voltage characteristic (I-V curve) can be obtained by sweeping the bias voltage over a broad range, say $-100 \mathrm{~V}$ to $+100 \mathrm{~V}$. Since the probe is being swept from the ion collection (negative bias) mode to the electron collection (positive bias) mode, information about the plasma ion density, $n_{i}$, and electron density, $n_{e}$, the electron temperature, $T_{e}$, the floating potential, $V_{f}$, and the plasma potential, $V_{p}$, can be found. A typical current-voltage characteristic is shown in Figure 2.9.

In a radio frequency plasma discharge such as the one investigated in this the- 


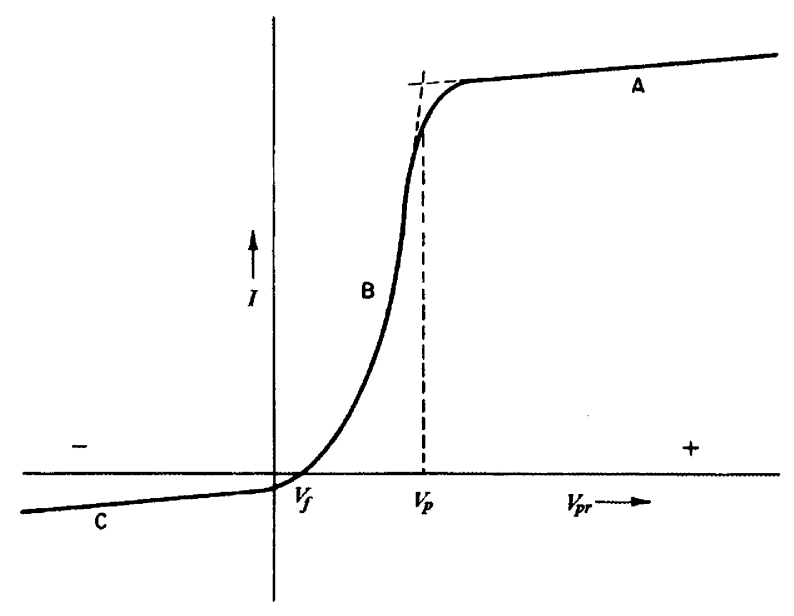

Figure 2.9: A typical Langmuir probe current-voltage characteristic.

sis, a RF signal can develop over the probe sheath because the capacitance of the sheath is dependent on the plasma potential, which fluctuates with the RF driving frequency and its harmonics (Sudit and Chen, 1994). Langmuir probes can be constructed with additional electronic components in the probe tip to compensate for the influence of the RF signal and allow the voltage measured by the probe to compensate for the fluctuating RF component of the plasma potential. Such probes are very difficult to manufacture and operate. In this thesis, the majority of the plasma diagnostic work is conducted with retarding field energy analyser (RFEA) probes, which are discussed in Section 2.6, since RFEA probes are particularly good at determining the plasma potential and the ion beam potential in RF plasmas. Therefore an RF compensated Langmuir probe is not required and a simple planar Langmuir probe, which is easier to construct and operate, is used in this work. This Langmuir probe is primarily used to determine the plasma ion density and a few measurements of the floating potential and the electron temperature are also made.

The plasma ion density is found by operating the Langmuir probe in the ion collection mode (Region C in Figure 2.9) and measuring the ion saturation current, $I_{\text {isat }}$. By applying a sufficently large negative bias, all the neighbouring plasma electrons are repelled and the measured current is a function of the ion flux through the sheath around the probe tip, such that

$$
I_{\text {isat }}=0.6 e A_{p} v_{B} n_{i}
$$

where $A_{p}$ is the collection area of the Langmuir probe tip and $n_{i}$ is the plasma 
ion density. $v_{B}$ is the Bohm velocity,

$$
v_{B}=\sqrt{\frac{k T_{e}}{m_{i}}}
$$

where $k$ is the Boltzmann's constant, $T_{e}$ is the electron temperature and $m_{i}$ is the ion mass and the factor 0.6 in Equation 2.1 approximates the drop in density caused by the acceleration, in the presheath, of the ions up to the Bohm velocity.

A floating object immersed in a plasma cannot draw any current and hence will obtain a potential such that equal fluxes of ions and electrons reach its surface. This potential is the floating potential, $V_{f}$, and corresponds to the point on the current-voltage characteristic where the curve crosses zero. In practice, the floating potential can be easily measured by leaving the Langmuir probe open circuit and recording the potential with respect to ground. The electron temperature is determined, as detailed by Lieberman and Litchtenberg (2005), by taking the gradient of a linear fit of the natural logarithm of the electron current, $I_{e}$, since

$$
\ln \left(\frac{I_{e}}{I_{\text {esat }}}\right)=\frac{V-V_{p}}{T_{e}}
$$

where $I_{\text {esat }}$ is the electron saturation current, $V$ is the bias voltage on the probe, $V_{p}$ is the plasma potential and $T_{e}$ is in volts.

\subsubsection{Probe Design and Installation}

Both Langmuir probes used in this thesis have a planar disk tip $3.0 \mathrm{~mm}$ in diameter as their collecting surface. One is a 'dogleg' probe and the other has a straight support tube. A cross sectional view of the dogleg probe is shown in Figure 2.10. When constructed the Langmuir probe consists of the following components, as shown in Figure 2.10: a planar disk probe tip (A), copper wire with enamel coating (B), stainless steel tubing $6.5 \mathrm{~mm}$ in diameter (C), heat shrink (D), ceramic insulator tubing $(E)$, narrow stainless steel tubing $(F)$, plastic insulator tubing $(G)$ and autocrete ceramic paste $(\mathrm{H})$.

The probes can be installed either axially or radially as shown in Figure 2.4. When installed axially the probe tip is positioned on the centreline of the space simulation chamber and HDLT source tube at $\mathrm{r}=0 \mathrm{~cm}$ and the probe tip is orientated perpendicular to the magnetic field lines. Both probes can be moved axially along the centreline. The straight probe can be moved from inside the source tube to the back wall $(z=83 \mathrm{~cm})$ of the space simulation chamber. With the dogleg probe the back wall of the chamber cannot be reached because the retraction of the probe is 
restricted by the ceramic tubing of the probe. Therefore measurements can only be made to $\mathrm{z}=53 \mathrm{~cm}$, that is $30 \mathrm{~cm}$ from the back wall of the space simulation chamber.

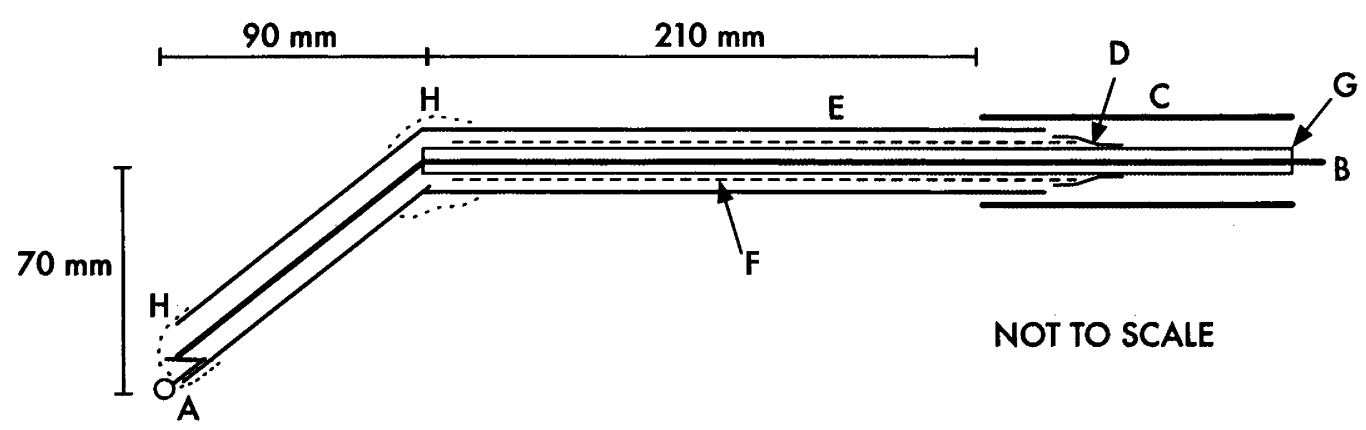

Figure 2.10: A cross sectional view of the dogleg Langmuir probe.

When installed radially, the dogleg Langmuir probe can be moved radially from $\mathrm{r}=0 \mathrm{~cm}$ to $\mathrm{r}=20 \mathrm{~cm}$ and can be positioned at various positions along the downstream portion $(z>3 \mathrm{~cm})$ of the space simulation chamber depending upon the access port used. The probe can also be moved from $\mathrm{r}=0 \mathrm{~cm}$ to the far side wall of the space simulation chamber $(\mathrm{r}=-50 \mathrm{~cm})$.

\subsubsection{Probe Operation and Data Analysis}

The local plasma ion density, $n_{i}$, is measured by biasing the Langmuir probe to $-73 \mathrm{~V}$, using a bank of $9 \mathrm{~V}$ batteries in series, and measuring the voltage drop, $V_{\text {meas }}$, across a known resistor, $R$. Here a $10 \mathrm{k} \Omega$ resistor is used. The ion saturation current is found by Ohm's Law, $I_{\text {isat }}=\frac{V_{\text {meas }}}{R}$, and substituted into Equation 2.1 to calculate the ion density. To measure $V_{f}$, the floating potential, the Langmuir probe is left open circuit and connected directly to the $1 \mathrm{M} \Omega$ input of a digital multimeter and the voltage recorded.

To determine the electron temperature, the I-V curve was obtained by sweeping the bias voltage on the Langmuir probe from $-100 \mathrm{~V}$ to $+100 \mathrm{~V}$. The bias voltage is supplied by a Hewlett Packard Bipolar Power Supply/Amplifier operating in the fixed gain amplifier mode and the sweep is controlled by a LabView program via a National Instruments NI DAQ Pad-6015 data acquisition system. The generated bias voltage is also read back to the LabView program by the NI DAQ Pad-6015 system via a voltage divider. The current on the Langmuir probe is measured using an Agilent 34401A 6 $\frac{1}{2}$ Digit Multimeter that is connected to the 
LabView program via a GPIB cable and interface card. The I-V curve is then analysed using a MATLAB program and a linear fit applied to the natural logarithm of the electron current, as per Equation 2.3, to determine $T_{e}$. The experimental uncertainty for all Langmuir probe measurements in this thesis is less than $10 \%$ unless otherwise stated.

\subsection{Retarding Field Energy Analyser}

\subsubsection{Principles of Operation}

A retarding field energy analyser (RFEA) probe is used to measure the ion current or the electron current as function of a discriminating voltage, $I_{i}\left(V_{d}\right)$ and $I_{e}\left(V_{d}\right)$ respectively, in the plasma produced by the HDLT prototype. The derivative of the collected ion current, $\frac{d I_{i}\left(V_{d}\right)}{d V_{d}}$, is used to determine the ion energy distribution function (IEDF) of the plasma ${ }^{\dagger}$. RFEA probes enable the separate study of negatively and positively charged particles. The main advantage over other electrostatic probes is the ability to separate ion and electron collection modes and to measure such particles in a specific direction, which provides a degree of spatial resolution. Therefore, charged particle beams can be investigated separately from the background plasma. RFEA probes can therefore yield information about the plasma potential, $V_{p}$, the ion beam potential, $V_{b}$, if present, and the plasma ion density, $n_{i}$. Although various designs have been developed over several decades (Neiswender and Kohout, 1972; Hopman et al., 1977; Bohm and Perrin, 1993; Conway et al., 1998), not as many theoretical studies of RFEA probes have been undertaken compared to the number for Langmuir probes. Several studies have also compared the plasma properties determined with Langmuir probes and RFEAs and have found good agreement between the two diagnostics (Ingram and Braithwaite, 1988; Gahan et al., 2008).

\subsubsection{Probe Design and Installation}

The RFEA probes used in this work are based upon the designs of Conway et al. (1998) and Charles et al. (2000) and have a standard configuration consisting

\footnotetext{
'Strictly speaking, $\frac{d I_{i}\left(V_{d}\right)}{d V_{d}}$ is not the IEDF; but since the integral of $f(v) d v=f\left(V_{d}\right) V_{d}=d n$, the ion velocity distribution can be found in terms of the discriminator voltage. This allows the IEDF to be computed experimentally from $\frac{d I_{i}\left(V_{d}\right)}{d V_{d}}$, which is a measurement of the energy of the ions entering the probe and falling through the sheath of the earthed analyser.
} 
of four grids and a collector plate. The analyser housing (Figure 2.11) is constructed from stainless steel and when assembled is $34 \mathrm{~mm}$ long, $19 \mathrm{~mm}$ wide and $11 \mathrm{~mm}$ deep. The housing is mounted on a $6.5 \mathrm{~mm}$ diameter stainless steel tube, which carries the electrical connectors for the grids, and is attached to a vacuum feedthrough. The analyser housing has two parts; the lid, which has two sides, forms a partial box into which the grid stacks are assembled. The analyser housing and support tube are both grounded.
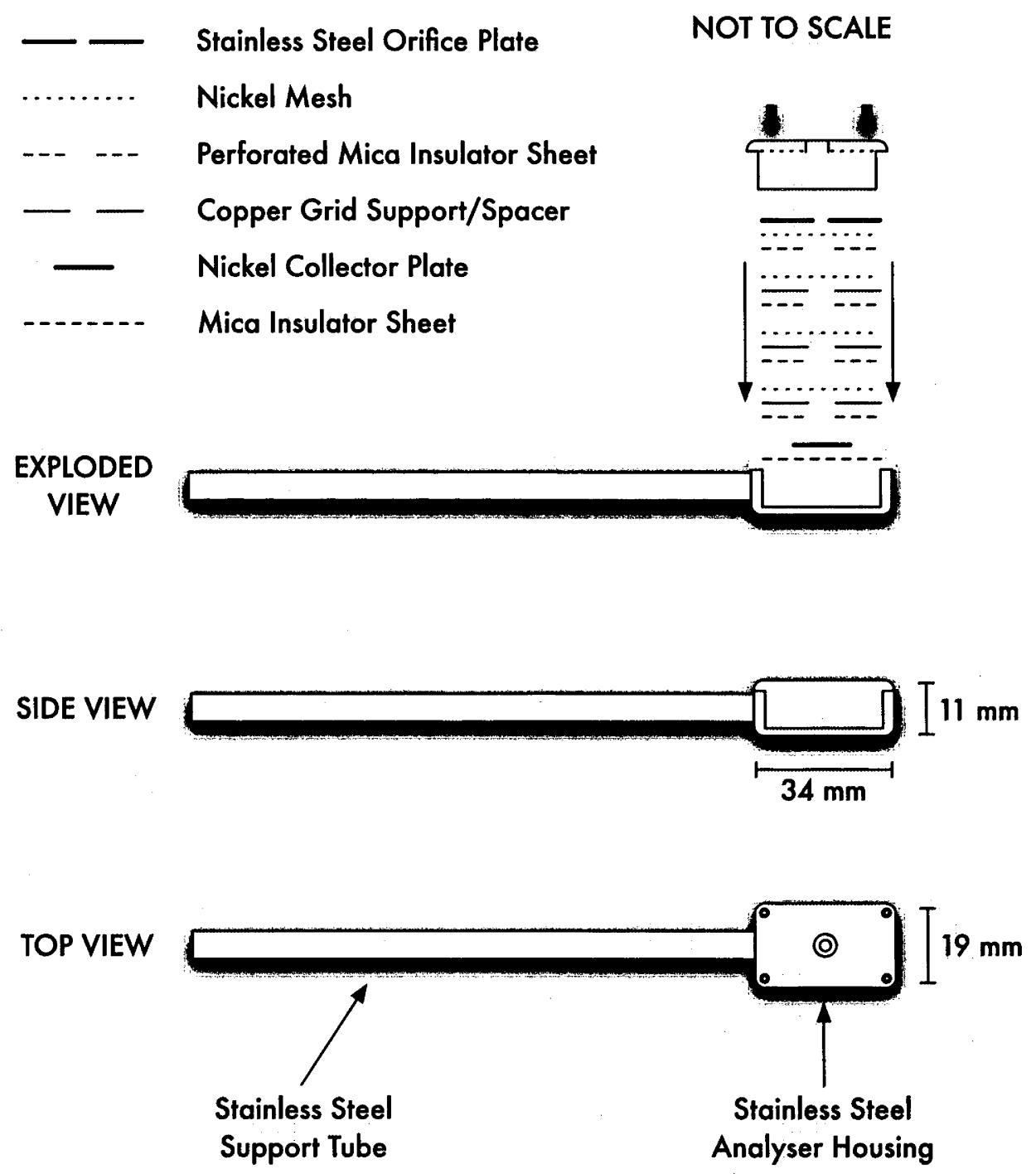

Figure 2.11: A cross sectional and exploded view of the RFEA probe.

The first layer of the grid stack is a $0.1 \mathrm{~mm}$ thick stainless steel orifice plate that is in electrical contact with the analyser housing and hence grounded to the space simulation chamber. The orifice plate has a $2 \mathrm{~mm}$ diameter aperture through which plasma particles enter the analyser. Following the orifice plate 
is an earthed entrance grid consisting of a layer of nickel mesh (approximately $58 \mu \mathrm{m}$ thick wires and a $882 \mu \mathrm{m}$ aperture; $88 \%$ transparency) then a $0.1 \mathrm{~mm}$ thick insulating mica sheet with a $6 \mathrm{~mm}$ diameter hole. The next three grid stacks, the repeller, discriminator and secondary emission suppressor, each consist of a layer of nickel mesh glued onto a $0.4 \mathrm{~mm}$ thick copper grid support/spacer, which is $10 \mathrm{~mm}$ square with a $7 \mathrm{~mm}$ diameter hole, and a layer of insulating mica sheet. The grid stack is completed by a nickel collector plate and mica insulating sheet and the whole assembly is clamped to the lid by a $1 \mathrm{~mm}$ thick stainless steel plate. Thin tags on one side of the copper supports extend beyond the clamp plate and provide electrical contacts for biasing the mesh grids. According to Bohm and Perrin (1993), the primary factor influencing the energy resolution is the geometry and grid separation of the RFEA probe. Conway et al. (1998) and Charles et al. (2000) have measured the energy resolution of an RFEA probe of this construction and in the presently investigated expanding plasma the resolution is better than $1 \mathrm{eV}$.

Two different RFEAs have been used in this work, one installed axially on the centreline of the HDLT and the space simulation chamber and the other installed radially as shown in Figure 2.4. The entrance orifice of the axial RFEA faces the HDLT exhaust which allows for simultaneous measurements of the local plasma potential $\left(V_{p}\right)$, ion beam energy $\left(V_{b}\right)$ when a beam is present and the total plasma density, $n$. The axial RFEA can be moved from the back wall of the space simulation chamber $(z=83 \mathrm{~cm})$ to the closed end inside the HDLT source tube $(\mathrm{z}=-26 \mathrm{~cm})$. The radial RFEA can be rotated upon its support tube axis so measurements can be made with the entrance orifice facing the HDLT exhaust $\left(\theta=0^{\circ}\right)$ or the chamber walls $\left(\theta=90^{\circ}\right)$. This RFEA can be installed at various axial locations, for example, at $60 \mathrm{~mm}$ from the exit of the HDLT source tube at $z=9 \mathrm{~cm}$; and can be moved radially from the HDLT centreline $(\mathrm{r}=0 \mathrm{~cm})$ to the wall of the space simulation chamber $(\mathrm{r}=50 \mathrm{~cm})$.

\subsubsection{Probe Operation}

Although the RFEA probe can analyse electrons and ions separately, in this work the analyser is mostly used in the ion-collection mode only. The bias circuit used is shown in Figure 2.12a and the voltages on the grids of the analyser are set at $0,-90,-18$ and $-9 \mathrm{~V}$ for the entrance grid, repeller grid, secondary emission suppressor grid, and the collector plate respectively (Figure 2.12b). The discriminator grid is located between the repeller and the secondary emission suppressor grid. A GW GPD-25H10 Regulated DC Power Supply is used to apply the voltage, $V_{R}$, to the repeller grid. This potential is sufficient to prevent 
most plasma electrons from entering the analyser during IEDF measurements. The discriminator voltage sweep, $V_{d}$, is provided by a Hewlett Packard Bipolar Power Supply/Amplifier operating in the fixed gain amplifier mode. The bias on the secondary emission suppressor grid is set to $-18 \mathrm{~V}$ in order to repel the secondary electrons emitted by the collector back towards the collector. Otherwise the secondary electrons would induce an additional ion current term on the collector (Bohm and Perrin, 1993). The small bias on the collector plate ensures that all ions are collected at the collector (Conway et al., 1998). Both of these two biases are supplied by batteries in the RFEA Amplifier Box.

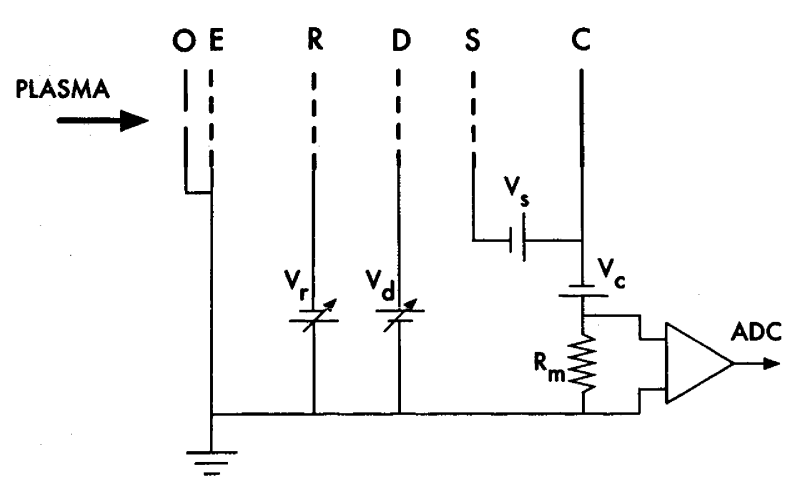

(a) Bias circuit

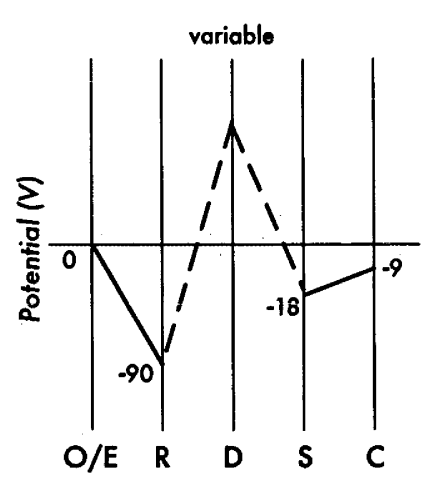

(b) Bias potentials

Figure 2.12: The RFEA biasing circuit and potentials: $\mathrm{O}$ - orifice plate, $\mathrm{E}$ - entrance grid, $\mathrm{R}$ - repeller grid, $\mathrm{D}$ - discriminator grid, $\mathrm{S}$ - secondary emission suppressor grid, $\mathrm{C}$ - collector plate.

The discriminator voltage sweep and data acquisition are controlled by a LabView program via a National Instruments NI DAQ Pad-6015 data acquisition system. The voltage on the discriminator grid is swept from 0 to $+100 \mathrm{~V}$ in $0.5 \mathrm{~V}$ increments by applying a staircase ramp to the amplifier input voltage. The RFEA Amplifier Box sums the collector current and the secondary emission suppressor grid current with 100 current measurements averaged at each voltage increment to produce a time-averaged ion current versus discriminator voltage curve $\left(I_{c}\right.$ vs $\left.V_{d}\right)$.

\subsubsection{Data Analysis}

The ion current is the integral of the ion energy distribution function from $v_{0}$ to infinity such that

$$
I_{c}\left(v_{0}\right)=e A_{r} G^{4} \int_{v_{0}}^{\infty} v f(v) d v
$$


where

$$
v_{0}=\sqrt{\frac{2 e V_{d}}{m_{i}}}
$$

and $A_{r}$ is the collecting surface, $G$ is the grid transparency and the power of 4 accounts for the transmission across the four grids, $v_{0}$ is the parallel velocity of the ion collected for a discriminator grid potential of $V_{d}, f(v)$ is the parallel ion energy distribution function at the entrance to the probe and $m_{i}$ is the mass of a positive ion. Typical $I_{c}$ vs $V_{d}$ curves are shown in Figures 2.13a and 2.13b (solid line). For all measurements $V_{d}$ is measured relative to the chamber ground. This data is analysed using a MATLAB program to determine the plasma potential, the ion beam potential (if present) and the plasma density. First, the measured $I_{c}$ vs $V_{d}$ characteristic is smoothed, if required, using a Savitzky-Golay smoothing filter (Savitzky and Golay, 1964). This filtering method has the advantage of preserving the features of the $I_{c}$ vs $V_{d}$ characteristic, which are usually flattened by other averaging techniques that use adjacent data points to smooth the data, such as applying a moving average. The IEDF is computed as proportional to $-d I_{c} / d V_{d}$ and so the smoothed $I_{c}$ vs $V_{d}$ characteristic is then differentiated to obtain the experimental IEDFs and normalised to 1 to facilitate comparisons, as shown as dashed lines in Figures 2.13a and 2.13b.

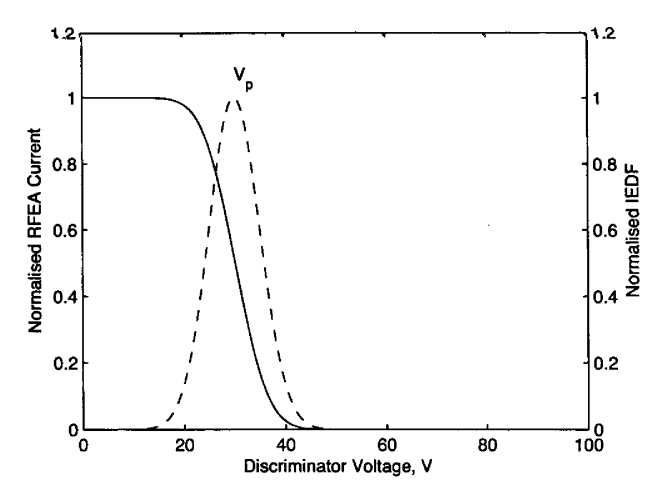

(a) A simple non-flowing plasma

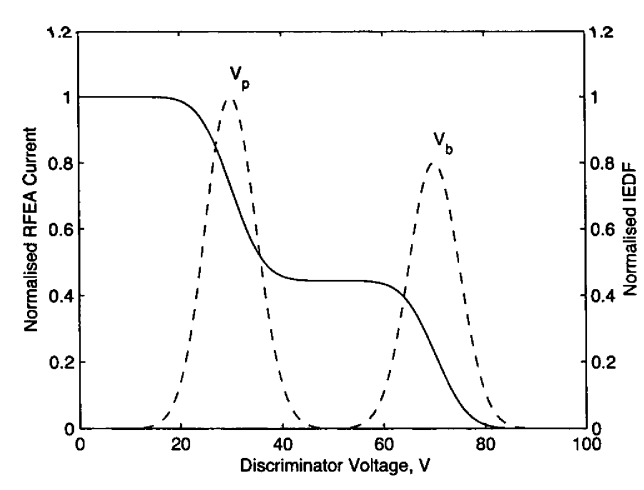

(b) An ion beam and background plasma

Figure 2.13: Normalised $I_{c}$ vs $V_{d}$ curve and ion energy distribution function.

For a simple non-flowing plasma, the experimental IEDF has a single peak, the centre of which corresponds to $V_{p}$, the local plasma potential at the probe location (Figure 2.13a). The width of the peak describes the parallel energy spread of the ions and the energy resolution. If an ion beam is present then a second peak is also present in the experimental IEDF (Figure 2.13b). The centre corresponds to the beam potential, $V_{b}$, and the width also describes the energy spread of the ion beam. To enable systematic analysis, the experimental IEDFs are fitted, 
as described by Charles and Boswell (2004a), with the sum of two independent Gaussian functions (as required). The quality of the fit is confirmed by integrating the sum of the Gaussians and fitting the result to the normalised $I_{c}$ vs $V_{d}$ curve. The centres of the Gaussian functions correspond to $V_{p}$ and $V_{b}$. When only one Gaussian function can be fitted, its centre corresponds to $V_{p}$ only. The experimental uncertainty on all the potential measurements in this work is $\pm 2 \mathrm{~V}$ unless otherwise stated and all potential measurements are relative to chamber ground.

The RFEA probe can be used to determine the plasma density, although most plasma density measurements in this work are made with a Langmuir probe, which is discussed in Section 2.5. To determine the plasma density, $n$, directly using the RFEA probe, the analyser was calibrated using a Langmuir probe in a high density plasma produced at a high pressure. This calibration method is commonly employed to calibrate RFEA probes (Sheridan, 2000; Charles et al., 2000). With the RFEA and Langmuir probes placed at $z=7 \mathrm{~cm}$ and the Langmuir probe biased at $-73 \mathrm{~V}$, so as to be in the ion collection mode, the ion saturation current is measured. The total current measured by the RFEA probe, $I_{c}\left(V_{d}=0\right)$, is also measured under the same conditions and then the RFEA probe is calibrated for plasma ion density measurements using the ion saturation current expression described earlier (Equation 2.1).

The total ion current, $I(0)$, collected by the RFEA corresponds to the integration over the whole ion velocity range $\left(v_{0}=0\right)$ and is found from the current measurement at zero discriminator voltage $\left(V_{d}=0 \mathrm{~V}\right)$ (Charles and Boswell, 2004a). The ion beam current, $I_{b}$, can also be determined and equals the current collected by the RFEA at the discriminator voltage that corresponds to the beam energy, $V_{b}$. If $V_{b}$ is constant then $v_{b}$ is also constant and $I_{b}$ yields the ion beam density, since $I_{b} \propto n_{b} v_{b}$.

The ratio of the ion beam density to the local downstream plasma density can also be determined from the RFEA measurement. This measurement gives an indication of what percentage of the plasma downstream is from the ion beam population and from the local plasma population and can be used to calculate the ion beam density, $n_{b}$. From Charles (2004), the ratio of the ion beam density to the local downstream plasma density is

$$
\frac{n_{b}}{n_{d s}}=\left(\frac{I_{b}}{I_{d s}+I_{b}}\right) \frac{v_{B}}{v_{b-d s}}
$$

where $I_{d s}$ is the current collected by the RFEA at the discriminator voltage corresponding to the local downstream plasma potential, $V_{p}$ and $v_{b-d s}=\sqrt{\frac{2 e\left(V_{b}-V_{p}\right)}{m_{i}}}$, 
the component of the ions velocity that comes from the difference between the potentials of the ion beam and the downstream plasma.

\subsection{High Voltage Probe}

To investigate the electrical properties of the matching box, a Tektronix P6015A $1000 \times 3.0 \mathrm{pF} 100 \mathrm{M} \Omega$ passive high voltage probe is used. The high voltage probe is able to measure DC voltages up to $20 \mathrm{kV}_{R M S}$ and pulses up to $40 \mathrm{kV}$ and has a bandwidth of $75 \mathrm{MHz}$ enabling the capturing of fast, high voltage signals. With the cover of the matching box removed, the probe tip is attached via an alligator clip to the part of the matching box circuit to be investigated and the grounding cable attached to the grounded copper matching box. The grounding cable is attached as close as possible to the measurement point in order to limit stray capacitances. The output of the high voltage probe is attached to a Hewlett Packard $54600 \mathrm{~A} 100 \mathrm{MHz}$ oscilloscope. With the plasma ignited and tuned to minimise the reflected power, the voltage trace is measured. Figure 2.14 shows a typical voltage trace acquired with the high voltage probe. From this measurement, the maximum and minimum voltages, $V_{\max }$ and $V_{\text {min }}$ respectively, the peak-to-peak voltage, $V_{p-p}$, and the voltage bias, $V_{b i a s}$, are determined as shown in Figure 2.14.

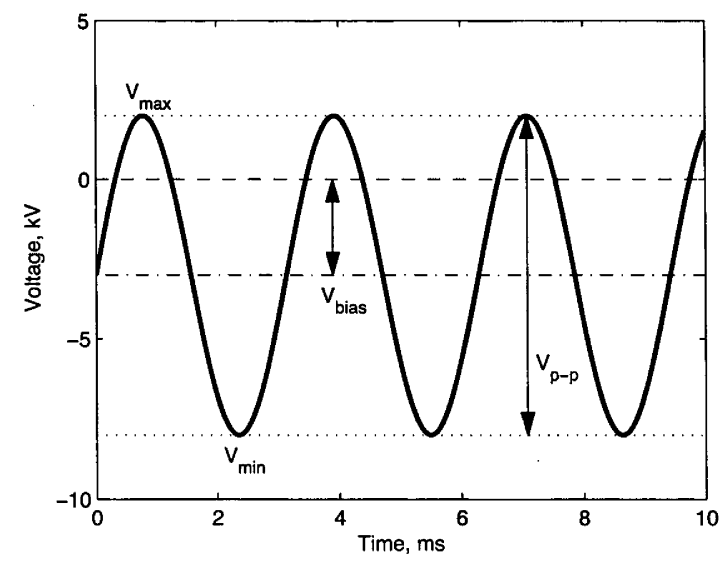

Figure 2.14: A typical voltage trace measured by the high voltage probe. 


\subsection{USB Spectrometer}

An Ocean Optics QE65000 UV/VIS spectrometer is used to investigate the emission spectrum from the plasma in the source and the exhaust of the HDLT prototype. The QE65000 spectrometer features a Hamamatsu back-thinned detector with a 2-D arrangement of pixels (1044 horizontal $\times 64$ vertical) that is responsive from $200-1100 \mathrm{~nm}$. The detector columns are binned, or summed, inside the detector prior to the readout process, thereby minimising readout noise. The detector is able to achieve up to $90 \%$ quantum efficiency with a high signal-to-noise ratio.

The QE65000 spectrometer is attached to a computer via a Universal Serial Bus (USB) interface and is controlled using the SpectraSuite software package. One end of an optical fibre is attached to the spectrometer unit and the other is placed against the window of the space simulation chamber and pointed at the region to be examined. Here measurements are made with the optical fibre positioned at the upstream window of the space simulation chamber focussing on the closed end of the HDLT source and from a side window focussing on the exhaust region downstream of the HDLT prototype. A collimator is used to prevent stray light from entering the optical fibre and measurements are made with the window ports of the space simulation chamber blacked out. A 20 millisecond integration time is used and the spectrum analysed using the SpectraSuite software package and MATLAB.

\subsection{Digital Oscilloscope}

When an oscilloscope is required, in most cases, a standard Hewlett Packard $54600 \mathrm{~A} 100 \mathrm{MHz}$ oscilloscope is used. However, for the measurements of the microarc discharges detailed in Section 5.2, a high sample rate oscilloscope is necessary to acquire a time-resolved measurement of the floating potential during a microarc event. These events generally occur on timescales of less than $1 \mathrm{~ms}$. A Tektronix TDS1012B Digital Storage oscilloscope is used since it has a sample rate of $1 \mathrm{GS} / \mathrm{s}$ with a bandwidth of $100 \mathrm{MHz}$. Measurements are made with the Langmuir probe open circuit and attached directly to the TDS1012B oscilloscope. The triggering is set so that when the floating potential becomes very negative ( $\sim-25 \mathrm{~V}$ for example) the data acquired over the preceding $0.5 \mathrm{~ms}$ and the following $1 \mathrm{~ms}$ is recorded to the data storage onboard the oscilloscope. The results are then plotted using MATLAB. 


\section{Momentum Flux Measuring Instrument for the HDLT Prototype ${ }^{\dagger}$}

\subsection{Principles of Thrust Measurement}

Thrust is the force produced by a rocket propulsion system acting upon a spacecraft. Thrust, along with specific impulse, is an important parameter used to evaluate the performance of a propulsion system and is used to compare different types of systems. Proper characterisation of the thrust levels for different operating modes of a particular system are an important part of space hardware qualification and enables spacecraft mission designers and planners to select the appropriate propulsion system for the desired application.

Electric propulsion systems, as discussed in Section 1.3, provide a high specific impulse, but low thrust, when compared to chemical propulsion systems. The latter produces thrust on the scale of $10^{6}$ Newtons, which is generally measured using load cells (Lee et al., 2000). Electric propulsion systems, however, produce thrust in the milliNewton range. As a result, the thrust of these systems are generally measured by a thrust balance - a device based upon techniques developed for laboratory mass scales.

Thrust balances used for electric propulsion devices typically measure the displacement caused by the recoil of the thruster when it is fired. The thruster is mounted on a floating structure that is connected to a fixed base via several sensitive flexures. The recoil of the thruster produces a rotational or linear displacement of the floating structure. A variety of means can be employed to measure the resulting displacement. Combining this information with knowledge of the

\footnotetext{
${ }^{\dagger}$ This chapter, in a slightly altered form, has been published in West et al. (2009b).
} 
properties and dynamics of the thrust balance, such as the spring constant of the flexures, allows the thrust to be determined.

\subsection{Thrust Measurement Techniques}

The force to be measured by a thrust balance is often thousands of times smaller than the force exerted by the mass of the thruster, which must be supported by the thrust balance. Electric propulsion systems also need to be tested in a vacuum environment and can produce considerable thermal loads on a thrust balance. Therefore, even though the basic principles remain constant, the specific requirements of a testing campaign often require the development of a custom thrust balance. Efforts to develop a single thrust balance that can operate over a large thrust range and can be used for a variety of different electric propulsion systems have resulted in very expensive and complex systems. Numerous different types of thrust balance are therefore in operation today. The main categories are hanging pendulums, inverted pendulums and torsional balances.

The simplest of the three configurations, the hanging pendulum (Figure 3.1a), is highly stable when subject to external perturbations but requires a long pendulum arm to obtain a high sensitivity. This limits the use of hanging pendulum thrust balances to large vacuum test facilities or those thrusters with a high thrust-to-weight ratio, $T / W$. For a hanging pendulum the displacement, $d$, is proportional to the applied force, $F$, by

$$
d=\frac{F l^{2}}{k}
$$

where $l$ is the length of the pendulum arm, $k$ is the spring constant of the pivot and $m$ is the mass of thruster. As a result, hanging pendulum thrust balances have mainly been used for MPD thrusters (Yoshikawa et al., 1984; Uematsu et al., 1985; Sasoh and Arakawa, 1993). A variation on the hanging pendulum is the long-period pendulum configuration. This uses a combination of linkages to produce the effect of a very long pendulum in a compact package. This is more complex than a simple hanging pendulum, but is required for impulse measurements where the period of the thrust balance must be longer than the duration of an impulse. Several possible configurations have been developed (Wilson et al., 1997; Polzin et al., 2006).

The inverted pendulum (Figure 3.1b) is the most popular design because of its simplicity and its use of gravity to increase sensitivity. The thruster is mounted 


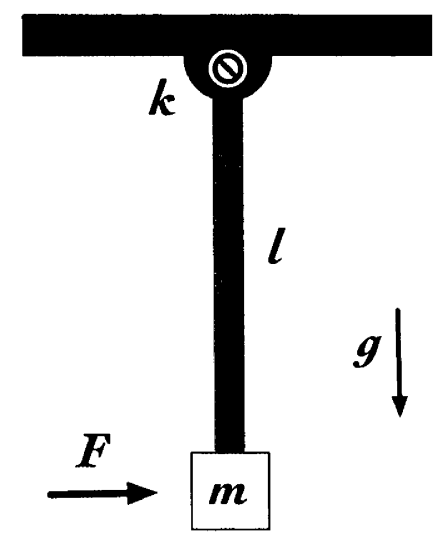

(a) A hanging pendulum

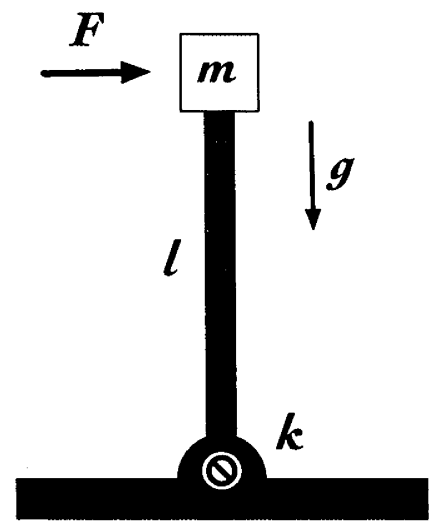

(b) An inverted pendulum

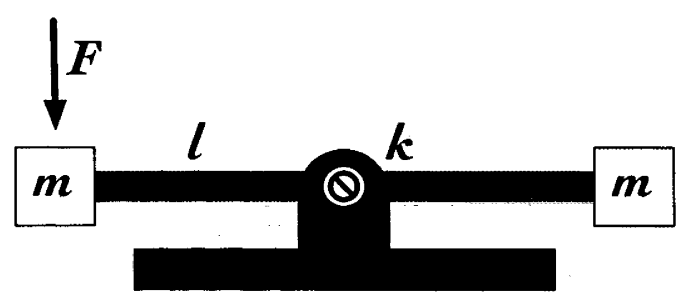

(c) A torsional thrust balance

Figure 3.1: The three most common thrust balance configurations.

on the end of a long arm and fires perpendicular to the arm, causing a small disturbance which is magnified by gravity to produce a measurable displacement. The sensitivity of the thrust stand is increased by applying the force from the pivot and the force from gravity against each other. Therefore, the displacement is related to the force by

$$
d=\frac{F l^{2}}{k-m g l}
$$

where $l$ is the length of the pendulum arm, $k$ the spring constant of the pivot and $m$ is the mass of thruster. Inverted pendulum thrust balances are particularly susceptible to thermal effects as their stability is dependent upon the stiffness of the supporting flexures, which can expand and contract during operation of the thruster (Kodys et al., 2003). Such balances often employ complex water or liquid nitrogen cooling systems to limit thermal drifts and deflections (Banetta et al., 2004; Polzin et al., 2006).

Torsional thrust balances (Figure 3.1c) allow an arm to swing freely around a pivot point at its centre. The thruster is placed at one end of the arm and a counterweight of mass equal to the thruster is placed at the other end, resulting in the mass of the thrust balance being perfectly centred on the axis of rotation. 
Therefore, this type of thrust balance is insensitive to all linear vibrations and two of the three components of rotational vibrations. Furthermore, the restoring force in this configuration is independent of the thruster mass, since the displacement can be described by

$$
d=\frac{F l^{2}}{4 k}
$$

where $l$ is the length of the pendulum arm and $k$ the spring constant of the pivot. Torsional thrust balances have the highest sensitivity of the three configurations and have been employed for testing various thrusters, including micropropulsion systems (Gamero-Castano, 2003). The primary disadvantage of this configuration is the horizontal, asymmetric arrangement required which is difficult to accommodate in vacuum chambers with limited space. The high sensitivity also limits its use with high power, steady-state thrusters (Polzin et al., 2006).

Other thrust measurement methods have been developed, principally for micropropulsion thrusters $\left(<10^{-6} \mathrm{~N}\right)$. These include electromagnetic thrust balances and methods that use moving platforms or frames (Nagao et al., 2007). Stephen et al. $(2001,2004)$, for example, use thin film strain gauges that measure the displacement of the platform in response to the recoil of the thruster. The electromagnetic thrust balance can not be used with thrusters that use magnetic fields, such as the HDLT, as the magnetic fields interfere with the operation of the thrust balance. Both methods are generally employed for thrusters that weigh less than 500 grams.

\subsubsection{Challenges Associated with the HDLT Prototype}

Several challenges are associated with using a thrust balance to measure the thrust produced by the HDLT prototype in its present configuration. The HDLT prototype, that is the HDLT structure, solenoids, source tube and antenna, weighs approximately $30 \mathrm{~kg}$ as the design has not yet been optimised to minimise mass. All of the above thrust measurement techniques are problematic for heavy thrusters because the expected thrust levels, in the milliNewton range, result in very small displacements when such high motion resistance must be overcome. These small displacements are very difficult to resolve accurately.

An attempt has been made to measure the thrust produced by the HDLT prototype using a hanging pendulum thrust balance. During the brief testing campaign at ESTEC, described earlier in Section 1.6.2, the HDLT prototype was mounted in the hatch in a fashion similar to the current configuration outlined in this thesis (Section 2.2). That is, with the HDLT structure attached to the rails 
on the roof of the chamber and the matching box connected to the antenna via the two copper conducting rods. These rods are covered by a RF shield that is attached to the side of the HDLT structure and internally to the side wall of the hatch.

A two-axis thrust balance was installed on the rails attached to the bottom of the hatch. The thrust balance, developed by Alta S.p.A in Italy under contract to ESA, used high precision fibre optic strain gauges based upon a Fabry-Perot interferometer (Banetta et al., 2004). The strain gauges are used to measure the strain on and hence the displacement of the arm of the pendulum. Liquid nitrogen was used to provide cooling and reduce thermal deformation. Given the mass of the HDLT prototype, the manner in which the RF power is connected and the rigidity caused by its mounting, only the source tube was mounted upon the thrust balance. This is possible because the source tube can move independently of the HDLT structure and the antenna of the thruster.

This approach assumes that the thrust produced by the HDLT prototype pushes against the source tube and not the antenna or magnetic field produced by the solenoid. Presently, it is not known what exactly the propellant mass expelled by the HDLT is pushing against (Fruchtman, 2006). Determining this is not trivial, has not been experimentally investigated before and is outside the scope of this work. Unfortunately, the thrust balance used at ESTEC was still in its commissioning phase and not operating correctly. Therefore no thrust measurements could be obtained during the brief testing campaign.

Other challenges also make measuring the thrust produced by the HDLT difficult in its current configuration. During operation, the HDLT prototype is placed under large thermal loads as the source tube, solenoids and antenna heat up. Since the thruster is operating inside a vacuum chamber, heat can only be dissipated via conduction or radiation, hence the large radiating panels that comprise part of the HDLT structure. If the HDLT prototype was mounted on a thrust balance, the whole system would be subject to time-varying thermal deformations and would require an elaborate thermal management system that adds complexity and is expensive to construct and operate.

The RF feedthrough, copper conducting bars and RF shielding, which are attached to the antenna and provide power to the HDLT prototype, are rigid and affix the HDLT prototype to the wall of the space simulation chamber. This configuration prevents the whole HDLT prototype from being mounted upon a thrust balance as the arm of the thrust balance and the HDLT prototype need to move independently of the space simulation chamber. The use of flexible cables to transmit the RF power from the matching box to the antenna is possible but 
very difficult. Such an approach would change the inductance of the antenna system and would require a redesign of the matching box and electrical system. This is undesirable and may still result in time-varying forces and torques being applied that could increase the uncertainty and noise in the measurements made by a thrust balance. Systems have been developed that conduct current on the thrust balance arm via pools of liquid mercury or gallium (Haag, 1991; Polzin et al., 2006). These materials can be hazardous and are difficult to work with. It is also not known whether such systems are able to conduct RF power at the frequencies or powers required to operate the HDLT prototype.

The design, construction, calibration and testing of a thrust balance, like that used at ESTEC, for use with the HDLT prototype is an expensive and very time consuming enterprise and is beyond the scope of this thesis. However, an alternative approach does exist which can be used to characterise the thrust produced by the HDLT prototype: the measurement of the momentum flux imparted to a target plate placed in the plasma plume (or exhaust) of the HDLT prototype.

\subsection{Previous Momentum Flux Instruments}

Various instruments have been developed previously to measure the momentum flux of plasma flows, diffusive plasmas and ion beams. A capacitance manometer has been used for momentum measurements in atomic and charged particle jets (Cohen et al., 1990). It is a complex device consisting of a refractory target plate attached by a long shaft to a thin, tensioned, circular, metallic diaphragm that is one electrode of a baratron pressure sensor capacitor. The shaft is suspended by two wires and the whole system behaves like a pendulum with the pressure transducer acting as a displacement sensor.

A compact cantilever force probe based on the pull-in phenomenon in microelectromechanical-system electrostatic actuators has been recently employed for plasma pressure measurements in the ISTTOK tokamak (Nedzelskiy et al., 2007). Ballistic pendulums have been employed for momentum measurements of laser-produced plasmas, which are of interest to the fusion research community, for many years (Grun and Ripin, 1982; Goncharov et al., 1988). These pendulums consist of a planar target, often made of Mylar or brass, suspended by nylon threads. Various techniques, including shadowgraphy and Hall effect sensors, have been used to determine the displacement of the pendulum and hence the momentum of the plasma flow. None of these systems adequately deal with the problems of electromagnetic interference and large temperature 
changes commonly produced by magnetoplasma thrusters or radio frequency driven plasmas, such as the HDLT prototype.

A double pendulum system, consisting of two identical plates suspended on razor sharp edges, has been used to determine the momentum flux and hence the thrust at the exhaust of a tandem mirror plasma thruster (Yang et al., 1995). The change in capacitance between the two plates was outputted as a voltage signal. The amplitude of the voltage signal was proportional to the impacting force or impulse from the plasma thruster. Thrust levels of approximately $75 \mathrm{mN}$ were measured in this case.

More recently, a target plate placed in the plasma exhaust of the VASIMR thruster, as shown in Figure 3.2, has been used to measure thrust levels on the order of 10s of $\mathrm{mN}$ (Chavers et al., 2006). Attached to the target plate is an alumina rod which is then fixed to a titanium beam on which four strain gauges are mounted. The force from the exhaust of the thruster on the target plate generates a torque, causing strain in the beam that is detected by the gauges, which are in a Wheatstone bridge configuration. A sensitivity of $0.1 \mathrm{mN}$ up to a force of $270 \mathrm{mN}$ is claimed (Chavers and Chang-Diaz, 2002).
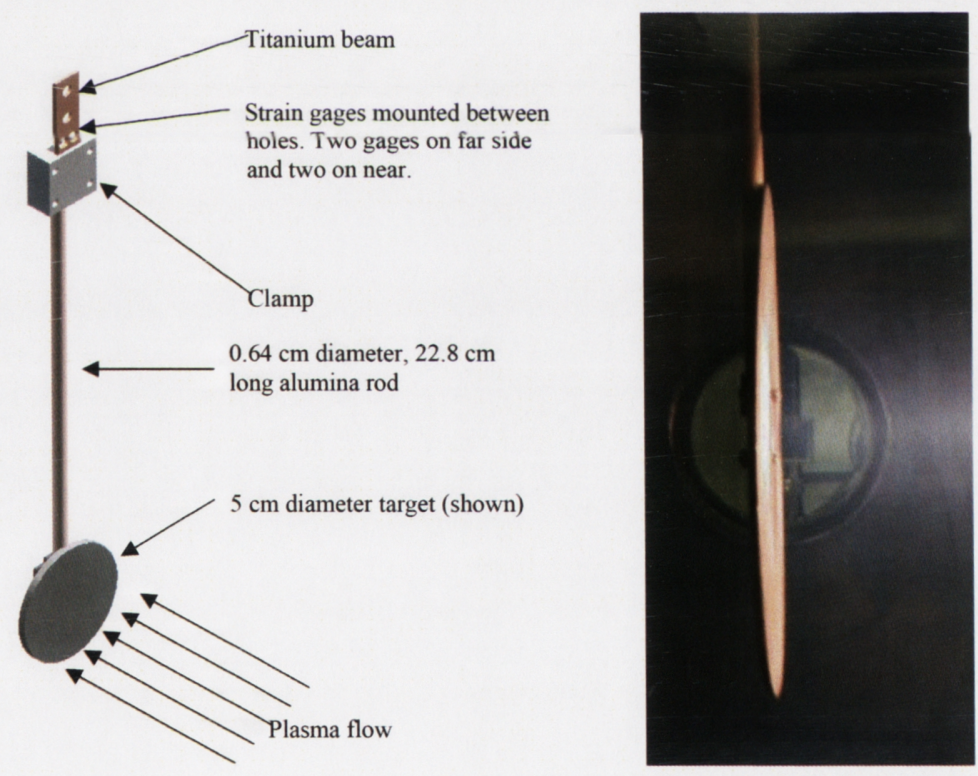

Figure 3.2: The momentum flux instrument developed for the VASIMR thruster. Image from (Chavers et al., 2006).

A recent study using a $5 \mathrm{~kW}$ xenon Hall thruster compared the results obtained with this instrument to that obtained with the thruster installed on an inverted pendulum thrust balance (Longmier et al., 2009). The results showed that the 
instrument had an average accuracy better than $98 \%$ compared to the thrust balance. However, this instrument uses semiconductor strain gauges that are very expensive, particularly difficult to mount and must be shielded from temperature changes and electromagnetic interference. An instrument based on that same design has also been adapted for plasma pressure measurements in the ISTTOK tokamak (Lunt et al., 2007). Makrinich and Fruchtman (2009) have also recently used a suspended target plate to measure the force exerted by the plasma flow from a radial plasma source.

\subsection{Momentum Flux Measuring Instrument Design}

A new Momentum Flux Measuring Instrument (MFMI) has been developed that is similar to that used with the VASIMR thruster but with some significant improvements. Given the difficulties posed by the use of strain gauges and Hall effect sensors outlined above, an alternative technique for measuring the displacement of a target plate has been developed. Previously, the materials science community has developed several techniques to measure the stress induced curvature or bending of thin film substrate combinations during plasma processing (Charles and Boswell, 1998). During the plasma assisted deposition of an amorphous thin film, the substrate is subject to deformation from tensile or compressive stress. This can be detected electrically or optically, but optical methods are preferred as they are not sensitive to the electric and magnetic fields or the charge up of the substrate. Systems developed to date use beam splitters (Bicker et al., 1998) and position sensitive detectors (Fitz et al., 2000).

Recently, a simplified technique has been developed and has been used to provide continuous stress measurements during film growth produced by a helicon activated reactive evaporation deposition system (Au et al., 2006). This technique dispenses with some of the mirrors and electronics required by previous systems. By adapting this optical deflection technique, the displacement of a target plate placed in a plasma thruster exhaust or diffusive plasma can be measured with a high sensitivity. This enables higher resolution momentum flux measurements of plasmas produced by electric propulsion devices and RF driven plasmas.

Laser interferometry and other systems using laser measurement techniques have also been employed to measure the displacement and dynamics of thrust balances. By placing the measurement system outside the vacuum chamber, the need for vacuum compatible optical components is removed and the problems associated with electromagnetic interference and thermal fluctuations are min- 
imised. This approach has been used for the measurement of thrust of several high-power steady-state plasma thrusters (Cubbin et al., 1997; Cassady et al., 2002; Kodys et al., 2006) and micropropulsion thrusters (Cubbin et al., 1997; Phipps et al., 2006; Kemp and Kovaleski, 2008; Li and Tang, 2005). High accuracy $(<2 \%$ error) measurements within a very wide thrust range (100 $\mu \mathrm{N}$ to above $10 \mathrm{~N}$ ) have been reported (Cubbin et al., 1997).

The new technique used in this thesis makes use of a simple and inexpensive target plate and places the measurement technique outside the vacuum chamber. Therefore, the laser displacement system does not need shielding from thermal effects, a vacuum compatible displacement sensor is not required and problems caused by vibration and electromagnetic interference are minimised. Since only one window is required to point the laser at the instrument target plate, this technique can be easily adapted to other apparatus if required. This approach does, however, require the target plate to be made from a vacuum compatible material.

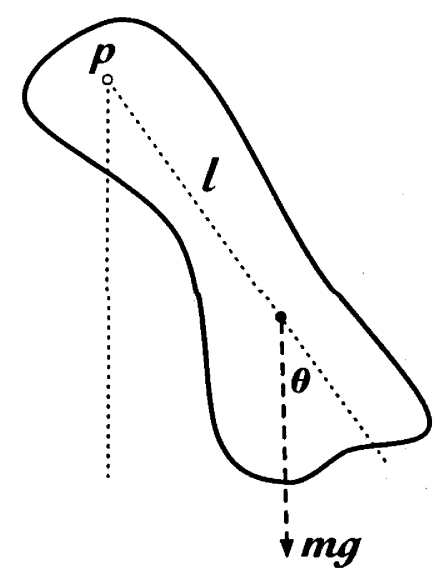

Figure 3.3: A compound pendulum with an arbitrary distribution of mass.

The MFMI is considered a compound pendulum. A compound pendulum, as shown in Figure 3.3, is a rigid body with a distributed mass that freely pivots around a horizontal axis which does not coincide with the centre of gravity of the system (Baker and Blackburn, 2005). A simple pendulum, in contrast, assumes that all the mass is concentrated at a single point. The equation of motion, as derived from Newton's Second Law, for a compound pendulum can be expressed as:

$$
I_{p} \frac{d^{2} \theta}{d t^{2}}+m g l \sin \theta=0
$$

where $I_{p}$ is the moment of inertia about the pivot point, $l$ is the distance from the pivot to the centre of mass, and $m$ is the mass of the pendulum. For small angular 
displacements, $\sin \theta$ equals $\theta$ and therefore the linearised equation of motion is:

$$
I_{p} \frac{d^{2} \theta}{d t^{2}}+m g l \theta=0
$$

The period of a compound pendulum can be derived and is equal to:

$$
T=\frac{1}{2 \pi} \sqrt{\frac{I_{p}}{m g l}}
$$

\subsubsection{Instrument Construction}

The MFMI, as shown in Figure 3.4, is a compound pendulum that consists of a target plate, which in this case is a silicon wafer. Two different diameter target plates were used in this thesis - a large silicon wafer $150 \mathrm{~mm}$ in diameter and a small silicon wafer $50 \mathrm{~mm}$ in diameter. The target plate is attached to an alumina rod - so it is electrically isolated - and then to a stainless steel tube $3.75 \mathrm{~mm}$ in diameter. The alumina rod slides inside the stainless steel tubing and is held in place with a small grub screw. This allows the length of the instrument to be adjusted as required and target plates to be interchanged easily if required.

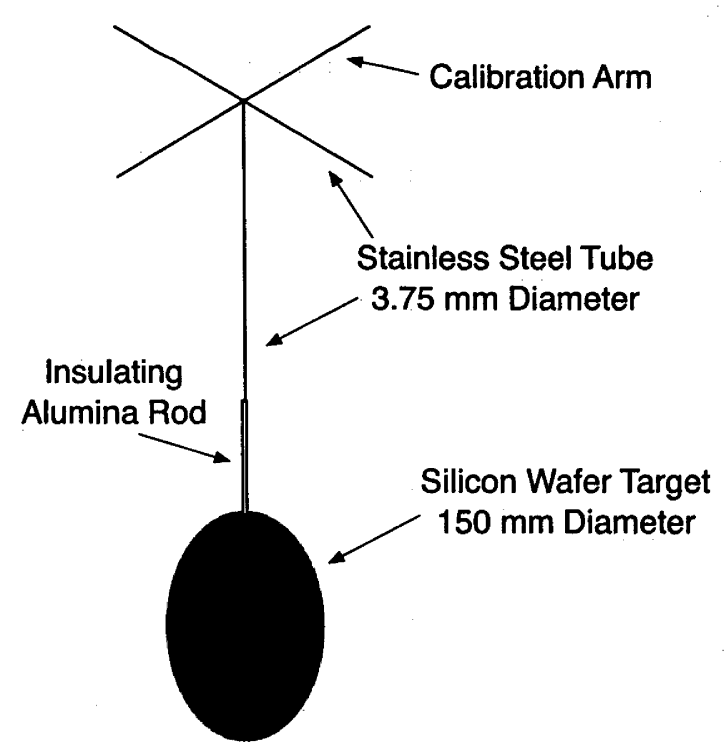

Figure 3.4: Construction of the MFMI.

Attached perpendicular to the tubing is a crosspiece also made from stainless steel tube $3.75 \mathrm{~mm}$ in diameter which pivots on two machined knife-edges affixed to the roof of the space simulation chamber. Another piece of stainless 
steel tube $3.5 \mathrm{~mm}$ in diameter is attached perpendicular to the cross piece and is $200 \mathrm{~mm}$ long. This piece provides a $100 \mathrm{~mm}$ moment arm on each side of the pivot point for calibration purposes. One side of the silicon wafer that is used as the target plate has a polished surface that can be used to reflect the laser beam. This allows non-intrusive measurement of the displacement of the instrument. The total mass of the instrument, that is the target, alumina rod and stainless steel tubing, was found to be $67.948 \pm 0.001 \mathrm{~g}$ when using the $150 \mathrm{~mm}$ target and $44.322 \pm 0.001 \mathrm{~g}$ when using the $50 \mathrm{~mm}$ target.

\subsubsection{Installation and Laser Measurement System}

The MFMI is placed on the two machined knife-edges that are suspended from the rails attached to the roof on the inside of the space simulation chamber described earlier in Section 2.2. Vibration isolation between the knife-edges and the vacuum chamber was considered, however, the vibration caused by the pumping system was found to be less than anticipated and negligible. The MFMI is positioned so that the target is $100 \mathrm{~mm}$ from the end of the HDLT source tube at $\mathrm{z}=13 \mathrm{~cm}$ as shown in Figure 3.5.

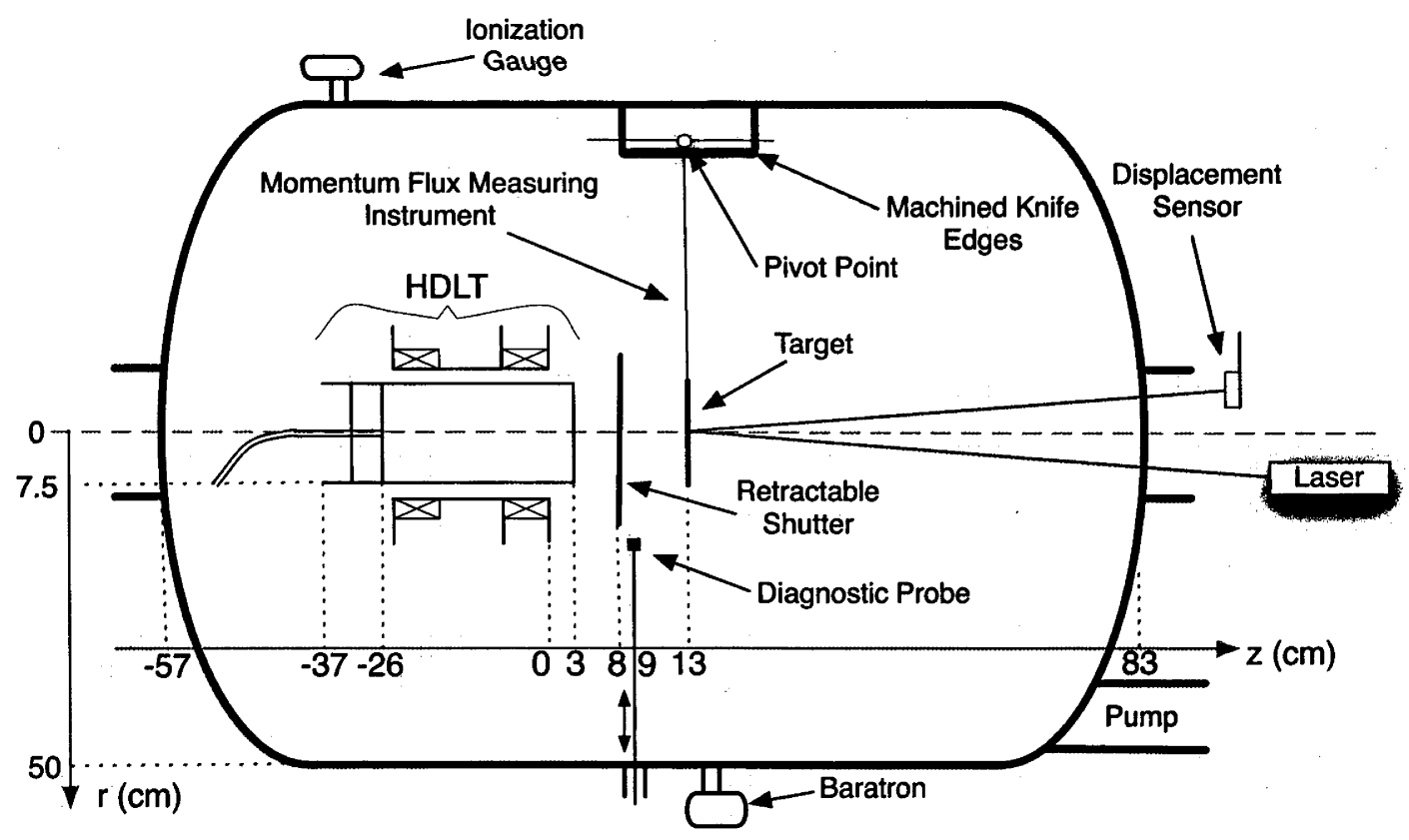

Figure 3.5: Schematic of the space simulation chamber from the side with the HDLT prototype, MFMI and laser measurement system installed. A diagnostic probe is also installed via a side port at $z=9 \mathrm{~cm}$. 
Using a method similar to that employed previously (Au et al., 2006), the displacement of the target plate is measured using a $1 \mathrm{~mW}$ He-Ne laser $(\lambda=633 \mathrm{~nm})$ pointed at the back of the silicon wafer target. The displacement of the reflected beam spot is measured using a THORLABS PDQ80S1 position sensing system that utilises a quadrant photodetector operating in the visible to near-IR range. The quadrant photodetector has a sensor diameter of $7.8 \mathrm{~mm}$ and is most sensitive when the beam diameter is between $1.0 \mathrm{~mm}$ and $3.9 \mathrm{~mm}$. A series of lenses focus the laser beam which then passes through a large window port at the downstream end of the space simulation chamber, reflects off the MFMI target, passes back through the window port and onto the position sensor. A USB interface sends the $\mathrm{x}$ and $\mathrm{y}$ coordinates of the centre of the reflected beam spot from the photodetector to a computer for data analysis.

\subsubsection{Minimising Unwanted Perturbations}

To minimise the oscillations caused by the surge of propellant striking the MFMI target when the propellant is injected prior to igniting the HDLT, a Millipore Tylan WGFG in-line filter is installed on the propellant line between the mass flow controller and the nylon tubing that feeds the propellant into the space simulation chamber and then into the HDLT source tube. In addition, a shutter, made from a disc of stainless steel sheet $1 \mathrm{~mm}$ thick and $160 \mathrm{~mm}$ in diameter, is placed between the end of the HDLT source tube and the MFMI target plate. The shutter, as shown in Figure 3.5, is located $50 \mathrm{~mm}$ from the end of the HDLT source at $z=8 \mathrm{~cm}$ and is attached to a stainless steel rod that enables the shutter to be retracted radially and removed from the HDLT exhaust plume. This rod includes a ceramic break that electrically isolates the shutter disc and ensures that it is floating. This allows for the propellant flow to be switched on, the HDLT to be ignited and the plasma to be formed without causing the MFMI to oscillate. Without these modifications, the instrument would undergo large oscillations that take several hours to damp out since it is moving in an evacuated volume and the only source of damping is the friction where the MFMI sits atop the machined knife-edges.

\subsection{Calibration}

To calibrate the MFMI known masses were added to the calibration arm of the MFMI, while the test facility was open and at atmospheric pressure, and the 
displacement of the target was measured using the laser displacement sensor. The displacement of the MFMI instrument was measured for several different masses. The range of masses used for the calibration of the two different configurations of the MFMI are shown in Table 3.1. The free body diagrams shown in Figure 3.6 are used to derive how the mass applied to the moment arm, $m$ can be related to the force on the target plate, $F_{t}$.

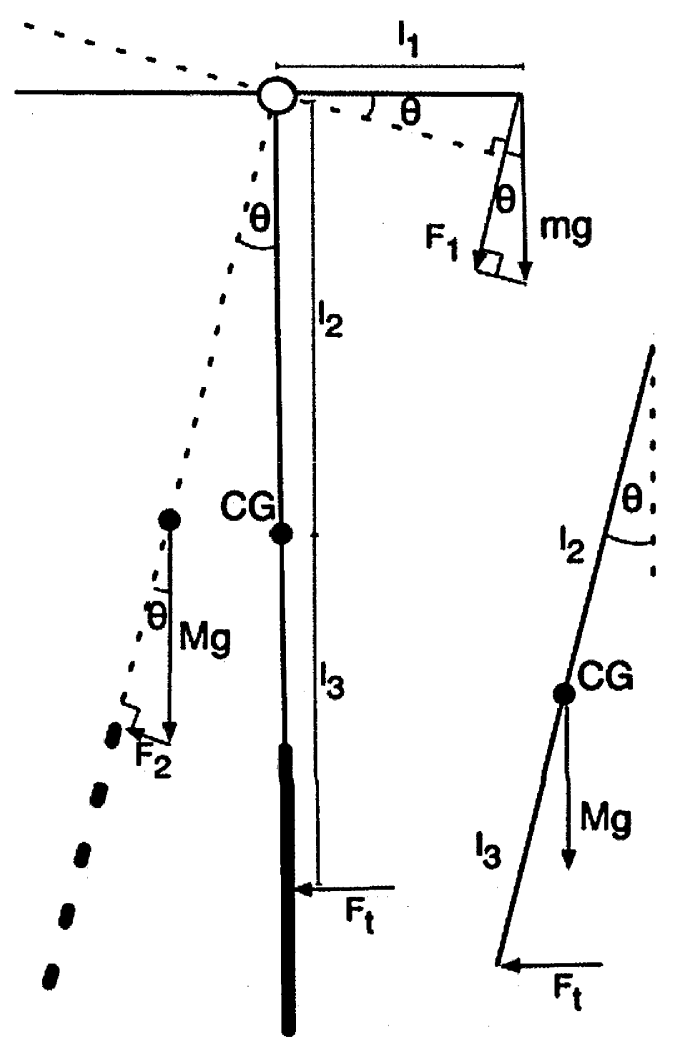

Figure 3.6: Free body diagrams for MFMI calibration. Main: Forces applied in relation to the pivot point of the MFMI. Inset: Forces applied in relation to the centre of gravity (CG) of the MFMI.

Since the moments around the pivot point $(\bigcirc)$ equal zero,

$$
\begin{gathered}
F_{1} l_{1}-F_{2} l_{2}=0 \\
m g \cos \theta l_{1}=M g \sin \theta l_{2} \\
\therefore \tan \theta=\frac{m g l_{1}}{M g l_{2}} \\
\therefore \theta=\tan ^{-1}\left[\frac{m g l_{1}}{M g l_{2}}\right]
\end{gathered}
$$


Table 3.1: Details for the calibration of the two MFMI configurations used.

\begin{tabular}{|c|c|c|}
\hline & Configuration A & Configuration B \\
\hline Target Diameter & $150 \mathrm{~mm}$ & $50 \mathrm{~mm}$ \\
Total Mass & $67.948 \pm 0.001 \mathrm{~g}$ & $42.322 \pm 0.001 \mathrm{~g}$ \\
$l_{1}$ & $100 \mathrm{~mm}$ & $100 \mathrm{~mm}$ \\
$l_{2}$ & $210 \mathrm{~mm}$ & $87 \mathrm{~mm}$ \\
$l_{3}$ & $226 \mathrm{~mm}$ & $363 \mathrm{~mm}$ \\
Calibration Masses Used & $8.57-177.27 \mathrm{mg}$ & $8.63-86.30 \mathrm{mg}$ \\
Force Range & $0-0.5 \mathrm{mN}$ & $0-0.2 \mathrm{mN}$ \\
Resolution & $15 \mu \mathrm{N}$ & $5 \mu \mathrm{N}$ \\
\hline
\end{tabular}

The moments around the centre of gravity $(\bullet)$ shown in the inset of Figure 3.6 also equal zero such that,

$$
\begin{gathered}
-M g \sin \theta l_{2}+F_{t}\left(l_{2}+l_{3}\right)=0 \\
\therefore F_{t}=M g \sin \theta \frac{l_{2}}{l_{2}+l_{3}}
\end{gathered}
$$

Combining Equation 3.10 and Equation 3.12 yields

$$
F_{t}=M g \sin \left\{\tan ^{-1}\left[\frac{m g l_{1}}{M g l_{2}}\right]\right\} \frac{l_{2}}{l_{2}+l_{3}}
$$

where $g$ is the acceleration due to gravity, $M$ is the total mass of the MFMI, $l_{1}$ is the length from the pivot point to the moment arm, $l_{2}$ is the length from the pivot point to the centre of gravity of the instrument and $l_{3}$ is the length from the centre of gravity to the centre of the target plate. The values of $l_{1}, l_{2}$ and $l_{3}$ used for the calibration of the two different configurations of the MFMI are shown in Table 3.1.

Using Equation 3.12 the deflection angle and displacement of the MFMI target from its equilibrium position can also be computed for a given applied force, $F_{t}$. For example, if $0.3 \mathrm{mN}$ of force was applied to the MFMI in configuration A, that is with the $150 \mathrm{~mm}$ installed, it would produce a deflection angle of approximately $0.05^{\circ}$ and a linear displacement of the MFMI target of approximately $0.4 \mathrm{~mm}$, which is barely discernible with the naked eye. It is worth noting that as the deflection angle increases the MFMI may become less sensitive to the change in the applied force. However, the forces required to achieve such increases in the deflection angle are well beyond the current range of forces measurable by the MFMI system, which is limited by the size of the reflected beam spot and the dimensions of the photodetector sensor. 
Figure 3.7 shows typical calibration curves for the MFMI and reveals a linear relationship between the force applied to the target, $F_{t}$, and the displacement of the laser beam spot, $\Delta y$, measured by the laser displacement sensor. $\Delta y$ is expressed in arbitrary units since the actual beam position relative to the true alignment can only be determined if the beam size, beam geometry and the beam power density are well characterised. This is not possible with the current experimental setup. As a result, the $y$-axis position determined by the quadrant photodetector is computed by taking the difference in the voltage measured by the top and bottom quadrants and is then expressed as a digital value between \pm 1500 by the software of the positioning system. When in the configuration with the $150 \mathrm{~mm}$ target plate, the MFMI is capable of measuring forces on the target of up to $0.5 \mathrm{mN}$ with a resolution of $15 \mu \mathrm{N}$. When using the $50 \mathrm{~mm}$ target, the MFMI is capable of measuring forces on the target of up to $0.2 \mathrm{mN}$ with a resolution of $5 \mu \mathrm{N}$.

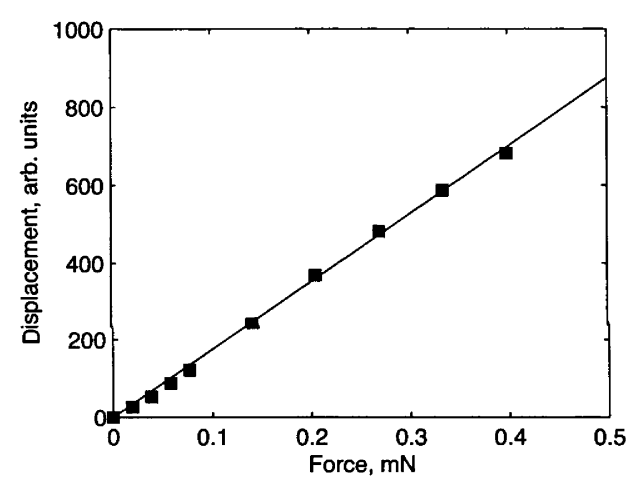

(a)

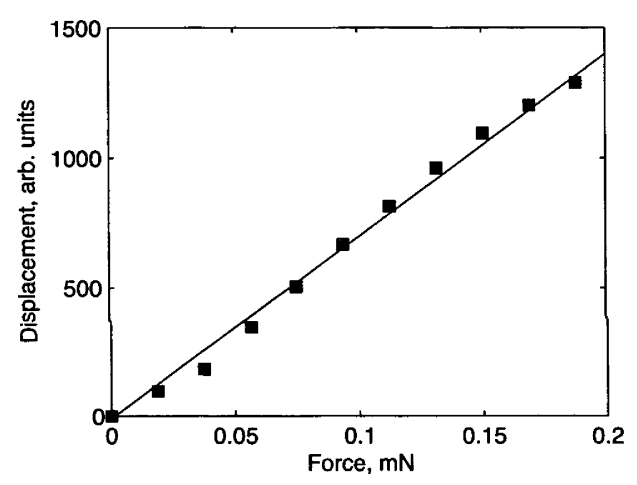

(b)

Figure 3.7: Typical calibration curves for the MFMI using (a) the $150 \mathrm{~mm}$ target and (b) the $50 \mathrm{~mm}$ target.

The resolution of the MFMI is limited by the resolution of the photodetector, the size of the beam spot that is reflected off the polished side of the target and the mass of the MFMI. Vibrations caused by the vacuum pumps and other equipment associated with the experiment, such as power supplies and cooling fans, could also limit the resolution of the MFMI yet were not found to be a concern in this case. The maximum force measured by the MFMI is limited by how much the MFMI can oscillate before it strikes the thruster and the range of displacements that can be measured by the laser measurement system. The latter can easily be increased if necessary by decreasing the size of the reflected beam spot using the focusing lenses or by using a photodetector with a larger sensor area. 


\subsection{Methodology and Data Analysis}

The reflected laser beam spot from the back of the MFMI target is aligned so that it is on the centre of the laser displacement sensor when the MFMI is at rest. Prior to recording data with the laser displacement sensor the equipment required to operate the HDLT is turned on, including the solenoid power supply, the RF generator and the SWR meter and oscilloscope that are used for monitoring the inputted RF power. These are switched on to ensure that any small vibrations caused by the operation of these devices are present throughout the measurement process at a known point in time.

With the shutter inserted to cover the open end of the HDLT source tube, the recording of data then commences. After 90 seconds, the propellant is injected and the RF power switched on to ignite the plasma. In the case where the response from only the propellant flow is measured, the procedure is identical except that the RF power is not switched on and no plasma is formed. The tuning on the HDLT matching box is adjusted to ensure the plasma is coupled correctly and sufficient RF power is deposited in the plasma. At 150 seconds, the shutter is retracted, which allows the plasma to strike the MFMI. The shutter remains retracted for 120 seconds, allowing the MFMI to oscillate and reach a new equilibrium position in response to the force applied to it by the plasma (or the propellant flow). At 270 seconds, the shutter is inserted again and the plasma is left on for a further 60 seconds, at which point the RF power and propellant flow are switched off. The laser displacement sensor continues to record data for a further 150 seconds. The total recording time is 480 seconds.

The MFMI, which is now oscillating as a result of the force from the plasma (or propellant flow), then requires approximately 2-3 hours to return to rest due to the limited friction in the system. Several devices were considered to dampen the oscillations and reduce the time between measurements but all were likely to result in movement of the MFMI from its intended position and affect the alignment of the reflected laser beam spot on the photodetector and the accuracy of the subsequent measurements.

Figure 3.8a shows an example of the raw y-axis displacement data collected by the laser displacement sensor versus time. Since the oscillation frequency of the MFMI is high, the data appears very noisy, yet the displacement of the MFMI is clear when the shutter is retracted at 150 seconds. To determine the displacement of the MFMI caused by the conditions investigated, the data is smoothed using a 10 point moving average. This filter window amounts to a 10 second period. This is greater than the period of oscillation of the MFMI which is about 4 seconds. 


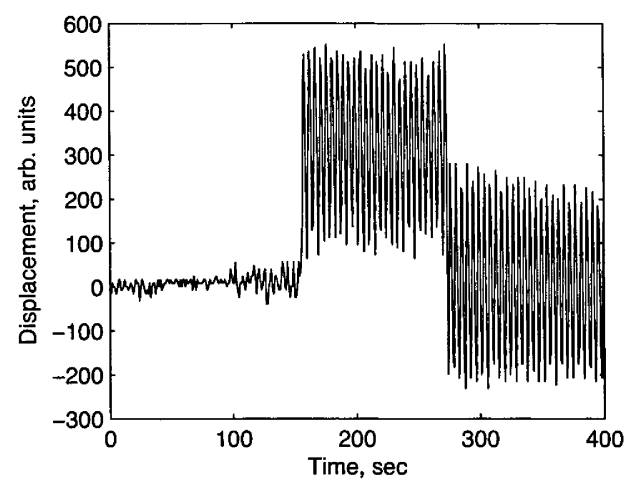

(a) Raw data

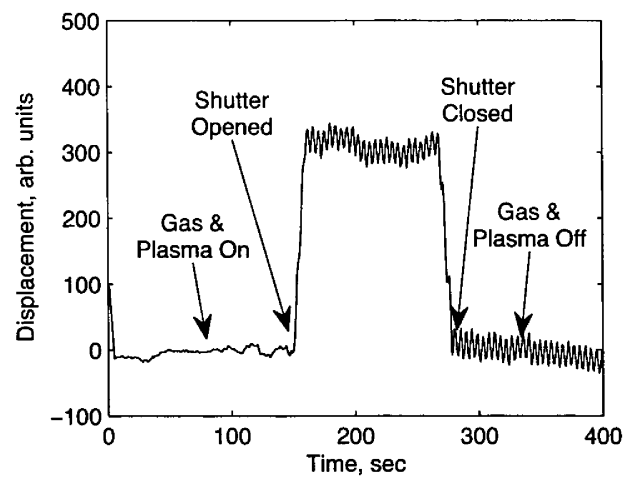

(b) Smoothed data

Figure 3.8: The data outputted from the laser displacement sensor.

Figure $3.8 \mathrm{~b}$ shows the data obtained following smoothing. This method has been applied to all data sets presented here.

To determine the displacement resulting from the conditions tested, $\Delta \mathrm{y}$ is the average displacement between $t=160$ seconds and $t=260$ seconds. This interval begins 10 seconds after the shutter is retracted, which allows for the MFMI to recover from the effect of retracting the shutter, and finishes 10 seconds before it is closed. In the example in Figure 3.8b, the mean is 309.1 units with a standard deviation of 10.7 units. Therefore, $\Delta y=309.1 \pm 10.7$ units. Using the calibration curve (Figure 3.7) the displacement measured is converted into the force measured on the target, $F_{t}$. For the case shown in Figure 3.8b, where $\Delta y=309.1 \pm 10.7$ units, $F_{t}=0.176 \pm 0.006 \mathrm{mN}$.

\subsection{Response to Cold Gas Flow}

Before investigating the force measured by the MFMI with the HDLT prototype operating, it is important to understand the forces acting on the target of the MFMI from the background pressure in the space simulation chamber and from the flow of neutral propellant exiting the thruster. Three cases are considered:

1. The forces applied by the background pressure with no directed flow.

2. The forces applied when an underexpanded sonic orifice is used to inject a propellant flow directly at the MFMI target.

3. The forces measured when the propellant flow is injected at the end of the HDLT source tube at a distance from the MFMI target. 
When no propellant flow is directed at the MFMI target yet the background pressure is increased, the background pressure exerts an equal force on the front and back sides of the MFMI target, $F_{b g}^{+}$and $F_{b g}^{-}$respectively, as shown in Figure 3.9a. Here $F_{t}=F_{b g}^{+}+F_{b g}^{-}=0$.

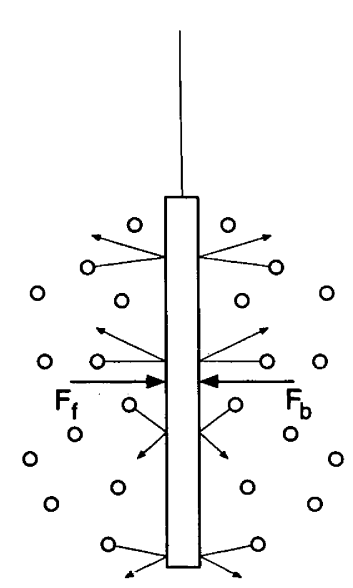

(a)

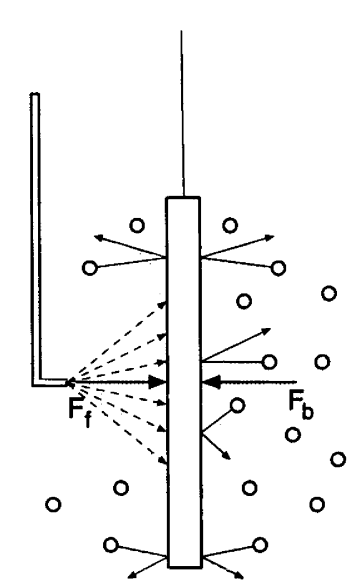

(b)

Figure 3.9: The forces acting on the MFMI target (a) from the background gas and (b) from the background gas and the jet from the underexpanded orifice.

To investigate the second case, measurements were made of the force generated by the cold gas flow through an underexpanded orifice placed close to the MFMI target. For these measurements, the MFMI was configured with the $150 \mathrm{~mm}$ target. A stainless steel tube, with one end attached to the mass flow controller that controls the propellant flow and the other attached to a machined orifice $3 \mathrm{~mm}$ in diameter pointing perpendicular to the centreline of the tube, was inserted radially through another side port on the space simulation chamber. It was positioned such that the orifice was $1 \mathrm{~cm}$ from the MFMI target at $\mathrm{z}=12 \mathrm{~cm}$ and at $\mathrm{r}=0 \mathrm{~cm}$ (i.e. on the HDLT prototype and space simulation chamber centreline). With the orifice facing away from the HDLT source and pointed at the MFMI target, measurements were made at various flow rates using argon. The results are shown in Figure 3.9b. These measurements also help to confirm the accuracy of the calibration outlined above.

In this case, the force on the target is $F_{t}=F_{j}+F_{b g}^{+}+F_{b g}^{-}$where $F_{j}$ is the force from the jet flow from the orifice. The jet flow is in the free molecular flow regime since the Knudsen number (Jamison et al., 2002), defined by

$$
K n=\frac{\lambda_{n}}{d_{o}}
$$




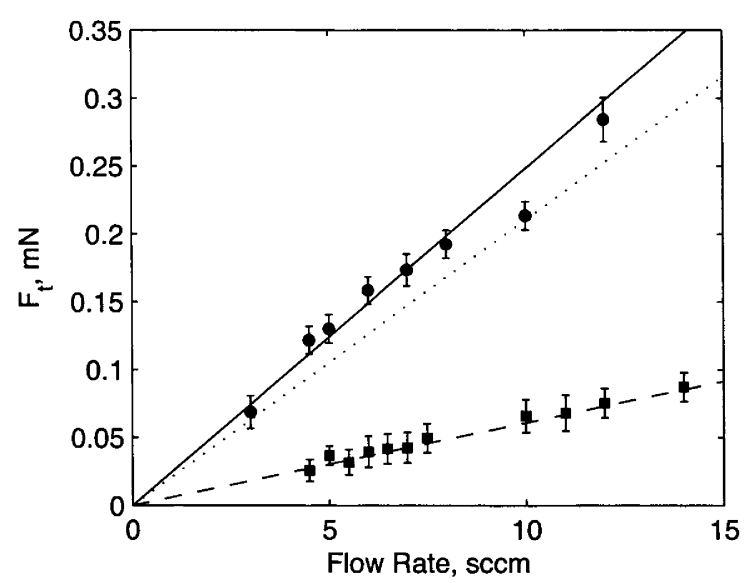

Figure 3.10: Force on the target from propellant flow using an underexpanded orifice (•) and with propellant injected at the closed end of the HDLT source tube ( $\square$ ). The solid line is the calculated value of $F_{j}$ when $\alpha_{j}=0.295$, the dashed line is the calculated value when $\alpha_{j}=0.0725$ and the dotted line is the calculated value when $\alpha_{j}=0.25$.

is high $(K n \geq 150)$ for these operating pressures (less than $1 \mathrm{mTorr})$. Here $\lambda_{n}$, the mean free path for argon neutral-neutral collisions, is greater than $50 \mathrm{~cm}$ and $d_{0}$, the diameter of the orifice, is $3 \mathrm{~mm}$. In the free molecular flow regime, individual particles seldom collide with each other and follow straight line trajectories and therefore the molecular flux density of the jet, per unit area, may, as described by Holkeboer et al. (1967), be computed as

$$
\Gamma_{j}=\alpha_{j} n_{n} \bar{v}_{n}
$$

where $\alpha_{j}$ is an empirical experimental gas transmission coefficient for the jet flow that is determined by a line of best fit and $n_{n}$ is the neutral gas density. The average velocity of the neutral gas particles, $\bar{v}_{n}$, is found from $\bar{v}_{n}=\sqrt{\frac{8 R T}{\pi M}}$, where $R$ is the ideal gas constant, $T$ is the gas temperature and $M$ is the molecular weight of the gas, which for an argon atom is $39.95 \mathrm{~g} . \mathrm{mol}^{-1}$ and for a xenon atom is $131.29 \mathrm{~g} \cdot \mathrm{mol}^{-1}$. The neutral gas density can be found from the ideal gas law, $n_{n}=\frac{p V}{k T}$. Since, $F=\bar{v}_{n} \frac{\Delta m}{\Delta t}$, the force from the jet flow is given by

$$
F_{j}=\Gamma_{j} A_{t} m_{n} \bar{v}_{n}=\alpha_{j} n_{n} A_{t} m_{n} \bar{v}_{n}^{2}
$$

where $A_{t}$ is the area of the MFMI target and $m_{n}$ is the neutral gas particle mass. Since $F_{b g}^{+}$and $F_{b g}^{-}$are equal, the force on the MFMI $F_{t}$ equals $F_{j}$. Figure 3.10 shows the results of measurements made for this case at varying flow rates. The force measured on the target $(\bullet)$ and the calculated value of $F_{j}$ when $\alpha_{j}=0.295(-)$ are shown. 
With the orifice removed, measurements were then made with the propellant flow injected at the end of the HDLT source tube. As mentioned previously and shown in Figure 3.5, the propellant line enters the closed end of the HDLT source at $\mathrm{z}=-26 \mathrm{~cm}$. The propellant line is made from nylon tubing with an internal diameter of $4.2 \mathrm{~mm}$. The propellant is injected $29 \mathrm{~cm}$ from the exit of the HDLT source and $39 \mathrm{~cm}$ from the MFMI target. The force on the target in this case is $F_{t}=F_{n}+F_{b g}^{+}+F_{b g}^{-}$where $F_{n}$ is the force from the neutral propellant flow injected into the HDLT source tube. Again, $F_{b g}^{+}$and $F_{b g}^{-}$are equal and

$$
F_{n}=\alpha_{n} n_{n} A_{t} m_{n} \bar{v}_{n}^{2}
$$

where $\alpha_{n}$ is an empirical experimental gas transmission coefficient for the injected propellant. In Figure 3.10 the force measured by the MFMI for different propellant flow rates is shown for this case ( $\mathbf{\square})$ and the calculated value of $F_{n}$ is also shown when $\alpha_{n}=0.0725(--)$.

As expected the force measured increases with increasing flow rate, however the magnitude of the force is significantly lower than that measured earlier using the underexpanded orifice placed $1 \mathrm{~cm}$ from the MFMI target, which was described above. This difference can be accounted for by the decrease in flux and velocity caused by the collisions that the neutral particles experience while traveling the $39 \mathrm{~cm}$ from the exit of the nylon tubing to the MFMI target and the diffusion of the neutral particles into the space simulation chamber once they exit the HDLT source tube. This decrease can be quantified by the ratio of the gas transmission coefficients for the two cases, $\frac{\alpha_{j}}{\alpha_{n}} \approx 4$. Therefore, the propellant injected through the underexpanded orifice positioned $1 \mathrm{~cm}$ from the MFMI produces four times the force than when the propellant is injected at the closed end of the HDLT source tube $39 \mathrm{~cm}$ from the MFMI. In summary, the force from the cold gas flow has been measured when the gas is injected close to the MFMI and from the upstream end of the source tube. The force produced by the cold gas has been shown to increase with flow rate in a repeatable fashion for both cases, as expected.

\subsection{Response to Plasma Discharge}

When the HDLT prototype is switched on and a plasma formed, the force on the MFMI target, $F_{t}$, is the sum of the force from the neutral propellant flow, $F_{n}$, and the force from the ions and electrons of the plasma impacting the target, $F_{p}$. Since the electrons are much lighter than the ions, the force from the electrons is considered negligible. The ions impacting the front side of the MFMI target consist 
of a population of slow ions and a population of fast ions from an ion beam (if present). The back of the MFMI target is impacted by the slow ion population only (Charles et al., 2007).

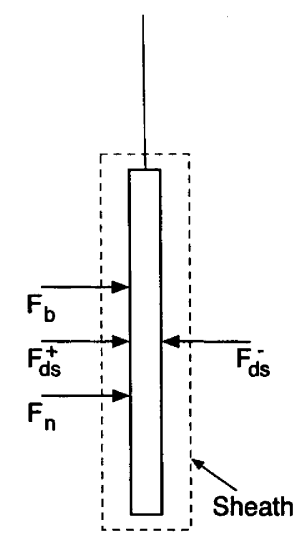

Figure 3.11: Forces acting on the MFMI target from the ion beam, the downstream plasma and the background gas when the plasma discharge is ignited. The sheath thickness is less than $1.5 \mathrm{~mm}$.

Ions in the local downstream plasma are accelerated by sheaths that form at both the front and back side of the MFMI target, as shown in Figure 3.11. The width of the sheath is less than $1.5 \mathrm{~mm}$. These local ions enter the sheath at the Bohm velocity, $v_{B}$, and the presheath acceleration potential is about $\frac{T_{e}}{2}$, which is much smaller than the sheath potential, $V_{s}$, which is approximately equal to the local plasma potential. The presheath acceleration is directed normal to the sheath and therefore we assume that the pitch angle of the local plasma ions impacting the target plate is zero, i.e. all the ions impact the target perpendicular to the surface of the target. The resulting opposite forces acting on the MFMI target can be written as

$$
\begin{aligned}
& F_{d s}^{+}=n_{d s}^{+} m_{i} A_{p} v_{B}^{2} \\
& F_{d s}^{-}=n_{d s}^{-} m_{i} A_{p} v_{B}^{2}
\end{aligned}
$$

where $n_{d s}^{+}$and $n_{d s}^{-}$is the density of the downstream plasma in front and behind the target plate, respectively and $m_{i}$ is the mass of an argon ion. $A_{p}$ is the area of the plasma that impacts the target plate.

In the case where an ion beam is present, these ions also impact the target plate. The force produced by the beam ions is given by

$$
F_{b}=n_{b} m_{i} A_{p} v_{b}^{2}
$$


where $n_{b}$ is the ion beam density and $v_{b}$ is the velocity of the beam ions which can be determined by

$$
v_{b}=\sqrt{\frac{2 e V_{b}}{m_{i}}}
$$

where $e$ is the electron charge and $V_{b}$ is the ion beam potential. Since the MFMI is positioned $10 \mathrm{~cm}$ downstream and the pumping speed in the space simulation chamber is low, the sheath in front of the MFMI is probably dominated by the large population of ions from the local plasma. The additional ion beam population originate from a region well upstream inside the HDLT source where the plasma potential is near $V_{b}$ (Charles et al., 1991, 1992). These ions suffer no collisions and acquire the full potential drop from $V_{b}$ to ground between that region and the target. Since the plasma is an expanding plasma, it is possible that these ions 'enter' the region at the exit of the HDLT source with a Bohm velocity. However, this would correspond to a potential drop of about $\frac{T e}{2}$, that is, only a few volts and is neglected in the calculation of $v_{b}$ from Equation 3.21. In the Chi Kung experiment, it has been shown that approximately $98 \%$ of the momentum of the beam ions produced is parallel to the centreline of the source (Cox et al., 2008a). Therefore, it is assumed that the beam ions here have a zero pitch angle, that is, the beam ions impact the target perpendicular to the surface of the target plate. The net force on the plate from the plasma, which can be determined from the plasma parameters just outside the sheath regions, is

$$
F_{p}=n_{d s}^{+} m_{i} A_{p} v_{B}^{2}-n_{d s}^{-} m_{i} A_{p} v_{B}^{2}+n_{b} m_{i} A_{p} v_{b}^{2}
$$

and therefore, as shown in Figure 3.11, the total force exerted on the target when the HDLT is switched on and a plasma formed is

$$
F_{t}=n_{d s}^{+} m_{i} A_{p} v_{B}^{2}-n_{d s}^{-} m_{i} A_{p} v_{B}^{2}+n_{b} m_{i} A_{p} v_{b}^{2}+\alpha_{n} n_{n} A_{t} m_{n} \bar{v}_{n}^{2}
$$

\subsubsection{Plasma Parameters Determined from Diagnostics}

In order to determine the parameters of the plasma that impacts the MFMI target, measurements are made with the Langmuir probe and RFEA described in Chapter 2. The Langmuir probe is installed radially, as shown in Figure 3.5, to measure the plasma density in front of and behind the MFMI target. The Langmuir probe can be moved radially from $\mathrm{r}=0 \mathrm{~cm}$ to $\mathrm{r}=20 \mathrm{~cm}$ and can be positioned $1 \mathrm{~cm}$ in front (at $\mathrm{z}=12 \mathrm{~cm}$ ) or $1 \mathrm{~cm}$ behind (at $\mathrm{z}=14 \mathrm{~cm}$ ) the MFMI target as desired. When biased negatively, so as to be in the ion collection mode, the ion densities $n_{d s}^{+}$and $n_{d s}^{-}$can be determined using the ion saturation current expression (Equation 2.1). 
The RFEA is also installed radially at $\mathrm{z}=9 \mathrm{~cm}$, that is, $60 \mathrm{~mm}$ from the exit of the HDLT source tube, and positioned in front of the MFMI target with the orifice facing the HDLT exhaust $\left(\theta=0^{\circ}\right)$. The RFEA can be moved radially from the HDLT centreline $(\mathrm{r}=0 \mathrm{~cm})$ to the wall of the space simulation chamber $(\mathrm{r}=50 \mathrm{~cm})$. The IEDF is obtained using the methods outlined earlier in Section 2.6 and the ion beam and local downstream plasma potentials, $V_{b}$ and $V_{p}$ respectively, are determined. The ions in the beam impact the MFMI target at a velocity dictated by the beam potential, therefore $v_{b}$ is found using Equation 3.21. From the RFEA measurement, the ratio of the ion beam density to the local downstream plasma density can also be determined as described in Section 2.6.

Using all of this information, the force from the plasma can be calculated from the parameters determined by the diagnostics and compared to the force measured by the MFMI. A comparison between the force measured with the MFMI and that calculated from the plasma parameters determined with the RFEA and Langmuir probe is provided in Section 4.9.

\subsection{Conclusions}

In this chapter, the principles underpinning the measurement of thrust produced by electric propulsion systems and the common techniques used have been detailed. Some of the challenges associated with using a standard thrust balance to measure the thrust produced by the HDLT prototype have been identified and an alternative approach - the use of a momentum flux instrument placed in the thruster exhaust - has been proposed. Previous instruments used for measuring the momentum flux from various plasma devices, including electric propulsion systems, have been reviewed and their advantages and disadvantages discussed in the context of implementing such systems for the HDLT prototype.

The design, construction and calibration of a new high sensitivity MFMI that uses a laser displacement system has been outlined. This instrument is designed specifically for the HDLT prototype and has been installed inside the space simulation chamber $10 \mathrm{~cm}$ from the open end of the HDLT prototype. It is capable of measuring force up to $0.5 \mathrm{mN}$ with a resolution of $15 \mu \mathrm{N}$ or forces up to $0.2 \mathrm{mN}$ with a resolution of $5 \mu \mathrm{N}$ depending on the size of the target plate used.

In this chapter, the response of the MFMI to the force produced from the cold gas flow of argon propellant has been investigated and shown to increase with the applied flow rate as expected. The forces acting upon the MFMI when a plasma is ignited have been derived along with their relationship to the measured plasma 
parameters. With this information, obtained from diagnostics placed in front and behind the MFMI target, it is possible to calculate the force imparted to the MFMI from the plasma and compare this with the force measured with the MFMI. In Chapters 4 and 6, measurements with a plasma discharge ignited using both argon and xenon propellant under various operating conditions, including in the high density xenon mode, are presented and compared with the results calculated from the measured plasma parameters at the location where the MFMI is installed.

The MFMI detailed in this chapter is a new diagnostic that has been developed for measuring forces in the exhaust region of electric propulsion devices. This system is a low cost method which compliments the use of conventional thrust balances. Since the laser displacement system is placed outside the vacuum chamber, the measurement approach used here is free from RF interference and thermal effects. This instrument could also be used to measure the thrust in the exhaust of other electric propulsion devices (Inutake et al., 2007; Blackhall and Khachan, 2007) and the momentum flux of ion beams formed by expanding plasmas (Corr et al., 2007, 2008) or fusion experiments (Goncharov et al., 1988). 


\section{Low Power Characterisation of the HDLT Prototype ${ }^{\dagger}$}

In this chapter, the first experimental evidence of a current-free double layer and ion beam formation with the HDLT prototype operating inside a space simulation vacuum chamber is presented. The plasma and ion beam created by the HDLT prototype when operating with argon is characterised axially and radially using the RFEA and Langmuir probe. For these measurements the propellant flow rate was maintained at $10 \mathrm{sccm}$, resulting in a pressure of $0.4 \mathrm{mTorr}$. This pressure is similar to that used in past experiments where current-free double layers have been found and is within the pressure range predicted by a model recently developed for current-free double layer formation (Lieberman and Charles, 2006). The influence of various operating parameters is investigated and measurements are made in the exhaust of the HDLT prototype with the MFMI. The ion beam created at low power when using xenon as the propellant is also investigated.

For all the experiments outlined in this chapter, with the exception of those in Section 4.7, the HDLT source tube was extended $3 \mathrm{~cm}$ beyond the HDLT structure, i.e. where $\mathrm{z}=3 \mathrm{~cm}$ is the end of the source tube. This is because in previous experiments when the HDLT prototype was attached to the Chi Kung vacuum chamber, the source tube also extended $3 \mathrm{~cm}$ beyond the HDLT structure. All measurements in this chapter are conducted with the plasma in a steady-state equilibrium and at $130 \mathrm{~W} \mathrm{RF}$ power to reduce thermal loading. The current applied to each solenoid was $3 \mathrm{~A}$, which produces a divergent magnetic field with an axial maximum of $138 \mathrm{G}$ at $\mathrm{z}=-5 \mathrm{~cm}$. The magnetic field topography has been described in Section 2.4.

\footnotetext{
${ }^{\dagger}$ Some of the results in this chapter have been published, in a slightly altered form, in West et al. (2008) and West et al. (2009b).
} 


\subsection{Ion Beam Detection}

All recent experiments that have detected ion beams formed by current-free double layers have consisted of a helicon plasma source attached to a larger diameter vacuum chamber. Simulation work by Meige et al. (2005b) has shown that an ion beam will still be generated when the grounded back wall of the vacuum chamber is reasonably distant $(\sim 80 \mathrm{~cm})$, however, experimental work is required to confirm this conclusion. Therefore, the detection of an ion beam with the HDLT prototype inside a larger volume space simulation chamber needs to be demonstrated first.

Figure 4.1 shows the $I_{c}$ vs $V_{d}$ characteristic and the IEDF obtained when the RFEA is located upstream, $5 \mathrm{~cm}$ inside the HDLT source at $\mathrm{z}=-2 \mathrm{~cm}$. The accuracy in the position of the RFEA is $\pm 2 \mathrm{~mm}$. No ion beam is detected upstream inside the HDLT source since only one Gaussian function can be fitted to the experimental data. $V_{p}=55 \mathrm{~V}$ and is the local plasma potential, referenced to the grounded chamber and the body of the RFEA probe, at that position and corresponds to the centre of this Gaussian function. This result corresponds well with measurements made at a similar pressure in Chi Kung with a different RFEA probe that was calibrated with a Langmuir probe (Charles, 2005a) and measurements made with a RF compensated Langmuir probe in the same device (Charles and Boswell, 2007).

With the RFEA located $10 \mathrm{~cm}$ downstream at $\mathrm{z}=13 \mathrm{~cm}$ the $I_{c}$ vs $V_{d}$ characteristic and the IEDF shown in Figure 4.2 are obtained. This position is chosen because past RFEA measurements in the Chi Kung experiment have been made at a similar distance from the exit of the source tube. In Figure 4.2a, the small increase in collector current in the region beyond $60 \mathrm{~V}$ in the $I_{c}$ vs $V_{d}$ characteristic is a result of secondary electron emission from the RFEA collector plate which is a common occurrence with RFEAs (Bohm and Perrin, 1993) and does not affect the analysis of the experimental IEDFs produced.

The IEDF in Figure $4.2 \mathrm{~b}$ shows a clear ion beam, represented by the second peak of the IEDF, which is centred at $V_{b}=55.5 \mathrm{~V}$. The centre of the first peak is the local plasma potential at the probe location and is $V_{p}=39.5 \mathrm{~V}$. Since RFEA discriminator voltages are measured relative to the chamber ground, the directed beam energy shown in Figure $4.2 \mathrm{~b}$ does not indicate a directed beam energy of $55.5 \mathrm{~V}$, but rather a beam energy of $16 \mathrm{~V}$ on top of a plasma potential of $39.5 \mathrm{~V}$. The resultant directed beam energy will be discussed further Section 4.4. Both $V_{p}$ and $V_{b}$ measured downstream are in good agreement with that measured in Chi Kung at a similar pressure with a calibrated RFEA (Charles, 2005a). The local 


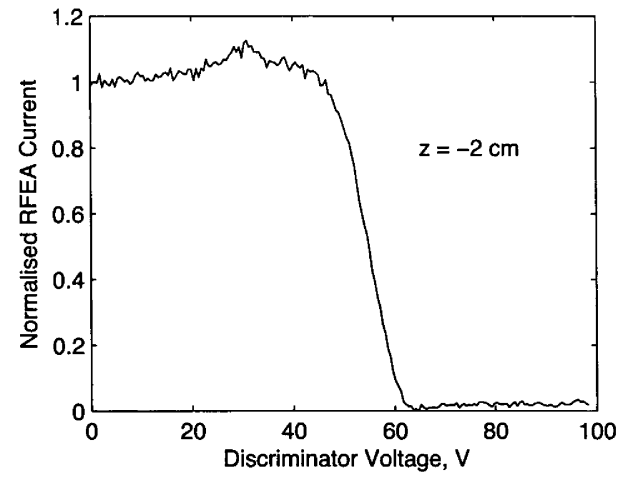

(a)

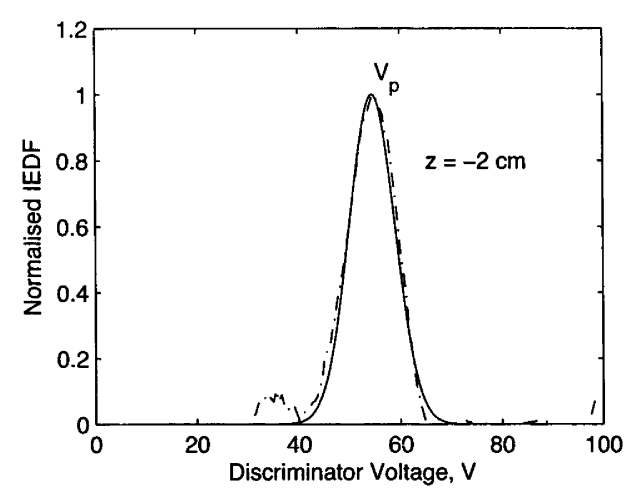

(b)

Figure 4.1: Measurements with the RFEA placed upstream at $\mathrm{z}=-2 \mathrm{~cm}$; (a) raw RFEA current and (b) normalised IEDF with raw data (-•) and Gaussian fits (-) shown.

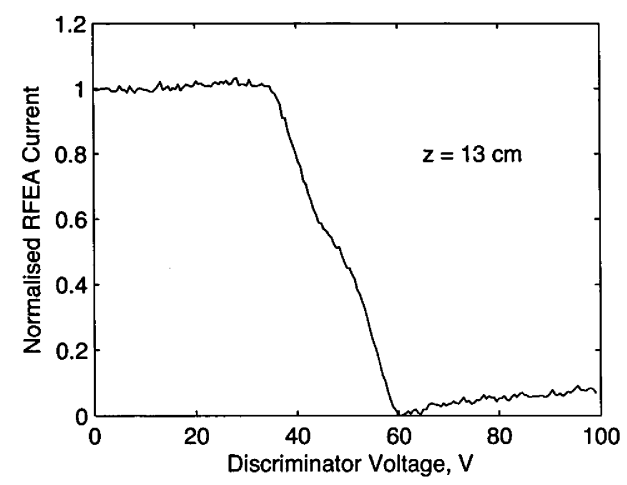

(a)

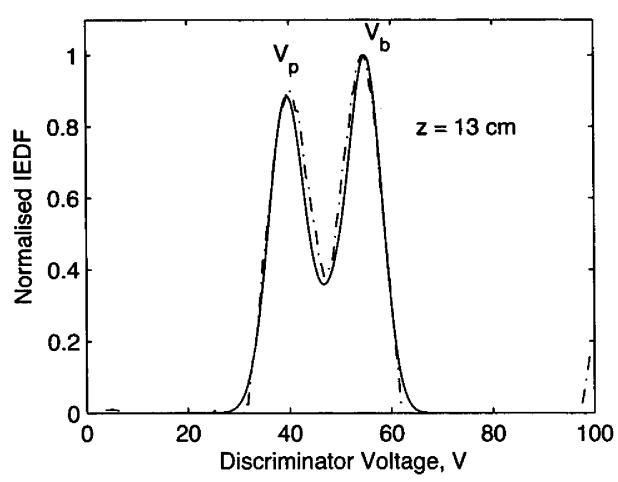

(b)

Figure 4.2: Measurements with the RFEA placed downstream at $z=13 \mathrm{~cm}$; (a) raw RFEA current and (b) normalised IEDF with raw data (-•) and Gaussian fits (-) shown. 
plasma potential inside the source, $V_{p}=55 \mathrm{~V}$, corresponds well, within the limits of the experimental error $( \pm 2 \mathrm{~V})$, with the ion beam energy downstream.

\subsection{Axial Plasma Characterisation}

Characterising the plasma produced by the HDLT prototype and its diffusion downstream into the space simulation chamber can provide some important information, such as the extent and nature of the ion beam and the electron temperature and density downstream. Axial potential and density profiles may also reveal the distinctive discontinuities associated with the double layer.

\subsubsection{Axial Plasma and Beam Potential Profile}

Figure 4.3 shows the potentials of the local plasma and the ion beam as a function of axial position for the operating conditions described above. The ion beam is detectable with the RFEA up to $21 \mathrm{~cm}$ downstream from the exit of the HDLT source at position $\mathrm{z}=24 \mathrm{~cm}$. Ion-neutral charge-exchange collisions are the main process involved in dampening the ion beam. For thermal ions in low-pressure plasmas, with ion temperatures, $T_{i}$, around $0.1 \mathrm{eV}$, the ion-neutral mean free path for argon (in centimetres) is

$$
\lambda_{i}=\frac{1}{n_{g} \sigma_{i}} \approx \frac{1}{330 p}
$$

where $n_{g}$ is the gas density, $\sigma_{i}$ is the total ion-atom scattering cross section for low-energy ions $\left(T_{i} \backsim 0.1 \mathrm{eV}\right)$ and $p$ is the pressure in Torr. A correction factor for the ion-neutral mean free path must be applied since the charge exchange cross section is velocity dependent (Lieberman and Litchtenberg, 2005). For a $15 \mathrm{eV}$ beam, the estimated effective mean free path is $\lambda_{b}=\lambda_{i} / 0.7$ (Lieberman et al., 2006). At $0.4 \mathrm{mTorr}, \lambda_{b} \sim 10 \mathrm{~cm}$ and therefore the ion beam could be measured for approximately two mean free paths before dampening by ion-neutral chargeexchange collisions merges the signal into the background.

From Figure 4.3, the local plasma potential downstream of the source drops off at a rate of approximately $13 \mathrm{Vm}^{-1}$ until it reaches the back of the space simulation vacuum chamber where the plasma potential measured with the RFEA is $\sim 28 \mathrm{~V}$. Using the Langmuir probe, the plasma potential measured at the back wall $(\mathrm{z}=83 \mathrm{~cm})$ is $\sim 26 \mathrm{~V}$. In both cases the measured potential is about $5 T_{e}$. 


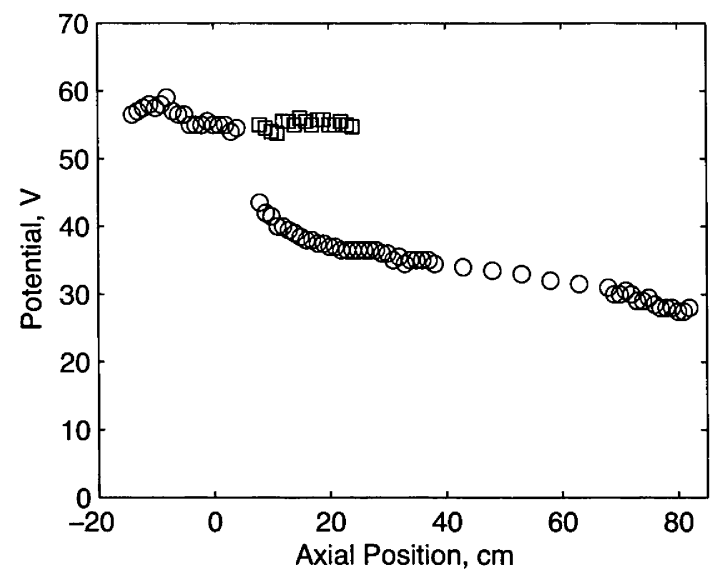

Figure 4.3: Local plasma potential, $V_{p},(\bigcirc)$ and ion beam potential, $V_{b},(\square)$ as functions of axial position.

These results confirm that the diagnostics are in good agreement and show that the back wall is adequately grounded.

Using the RFEA facing the HDLT source it has been difficult to determine the precise location of the characteristic local plasma potential discontinuity that is associated with the location of the double layer. However, it is present somewhere in the region between $1 \mathrm{~cm}$ and $5 \mathrm{~cm}$ from the exit of the HDLT source. Interestingly, the maximum of the magnetic field gradient is located $1 \mathrm{~cm}$ inside the HDLT source, so the double layer is located near the region where the magnetic field expands rapidly.

In an attempt to diagnose the location of the double layer, an axially installed RFEA with the entrance orifice facing radially was used as it only measures the thermal ion population and not the ion beam population at the higher potential. Figure 4.4 shows the local plasma potential measured with this RFEA as a function of axial position at 0.15 mTorr, $0.4 \mathrm{mTorr}$ and $1.0 \mathrm{mTorr}$. Interestingly, the potential discontinuity characteristic of the double layer is not observed even though it is clear from the IEDFs obtained with the source facing RFEA that an ion beam is present downstream. This result has been observed elsewhere (Corr et al., 2007; Takahashi et al., 2008a; Lafleur et al., 2009) and the most likely explanation is that when the grounded RFEA is inserted into the fully insulated HDLT source it perturbs the plasma such that the discontinuity, if present, cannot be measured. As an aside, Figure 4.4 also shows how the potential in the source increases with decreasing pressure while the potential downstream remains reasonably constant with pressure. 


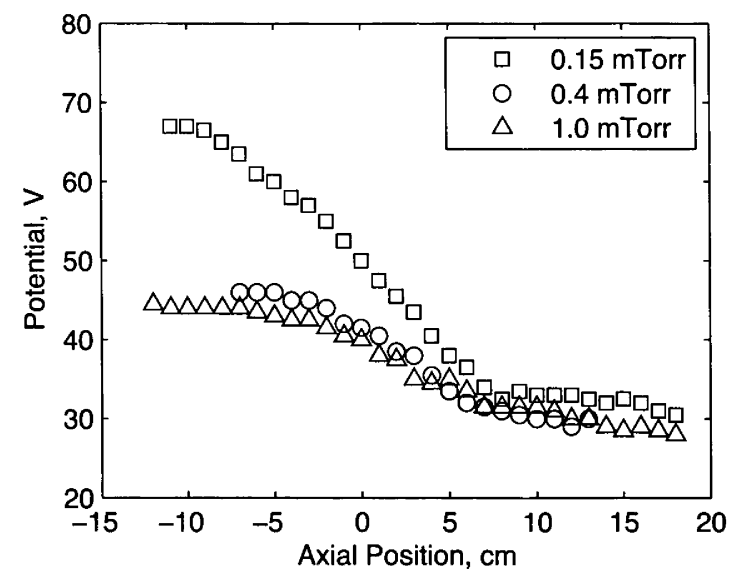

Figure 4.4: Local plasma potential, $V_{p}$ as a function of axial position at various operating pressures.

\subsubsection{Axial Density Profile}

The density profile is another important plasma characteristic and can be used to compare the results for this experiment with other investigations. The Langmuir probe was used to determine the density profile for the conditions described above ( $10 \mathrm{sccm}, 0.4 \mathrm{mTorr}, 130 \mathrm{~W}$ and $138 \mathrm{G})$. Figure 4.5 shows the plasma density, $n$, as a function of axial position. The plasma density upstream decreases from a maximum at $\mathrm{z}=-13 \mathrm{~cm}$ of $\sim 1.3 \times 10^{17} \mathrm{~m}^{-3}$ to $\sim 3.5 \times 10^{16} \mathrm{~m}^{-3}$ at the end of the HDLT source tube $(\mathrm{z}=3 \mathrm{~cm})$. The plasma density in the downstream region is reasonably constant around $\sim 1.0 \times 10^{16} \mathrm{~m}^{-3}$ in the region from $\mathrm{z}=12 \mathrm{~cm}$ to $z=32 \mathrm{~cm}$. This is also the region in which the ion beam is detected with the RFEA. As the plasma diffuses downstream the plasma density decreases as a result of the expansion until reaching the back of the space simulation chamber $(\mathrm{z}=83 \mathrm{~cm})$ where the density is $\sim 1.0 \times 10^{15} \mathrm{~m}^{-3}$. For comparison, Figure 4.5 also shows the axial density profile for an expanding plasma formed by the HDLT prototype at higher pressure (1.31 mTorr) with a magnetic field three times lower where the maximum $B_{z} \sim 45 \mathrm{G}$. The plasma density beyond $z=3 \mathrm{~cm}$, that is beyond the end of the HDLT source tube, decreases linearly on the logarithmic scale. This indicates that the plasma density for this case is decreasing exponentially with axial position, consistent with an expanding plasma and the assumption of a constant electron temperature.

The density profile measured with the Langmuir probe and shown in Figure 4.5 is consistent with the current-free double layers measured via Langmuir probe and other diagnostics in previous experiments with 'standard' configurations such as Chi Kung (Charles and Boswell, 2003). The densities on both sides of the 


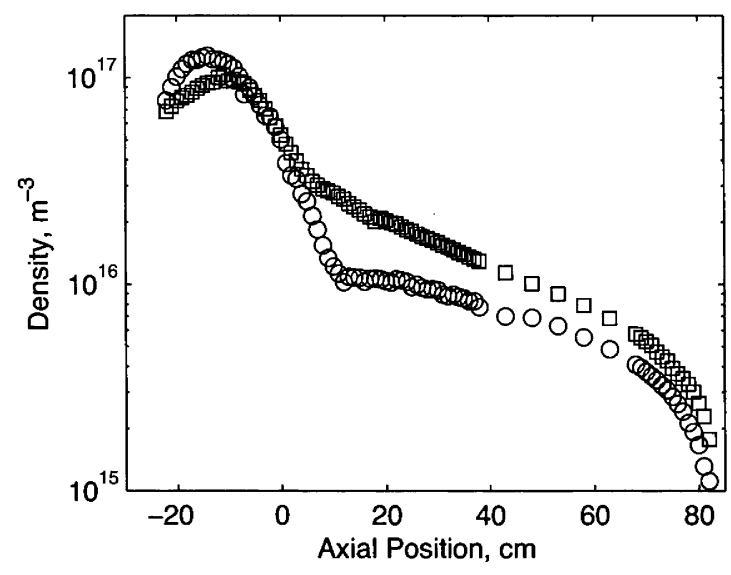

Figure 4.5: Plasma density, $n$, as function of axial position; for standard conditions $(\bigcirc)$ and for an expanding plasma formed at 1.31 mTorr with a low magnetic field ( $\square$ ).

double layer and the general shape of the plasma density profile conforms with the theoretical model for low-pressure current-free double layers developed recently (Lieberman et al., 2006). The experiments undertaken in Chi Kung (Charles and Boswell, 2003) reveal a small discontinuity in the thermal ion population density measured with a RFEA facing radially at the location of the double layer and simply a change of slope in the total ion density measured with the Langmuir probe near the double layer position. No such discontinuity is observed here. Nonetheless, the density profile obtained here is consistent with that found in Chi Kung and other experiments exhibiting double layers.

\subsubsection{Electron Temperature Downstream}

As previously observed in low pressure RF plasmas, the current-voltage characteristic measured axially in this experiment with the Langmuir probe deviates from the standard current-voltage characteristic (Figure 2.9) with the collected current in the electron saturation region increasing significantly, rather than levelling off, as the bias voltage is swept. Nevertheless, a quick analysis of some of the characteristics obtained axially yielded an electron temperature consistent with measurements in other experiments. Although non-RF compensated Langmuir probes are known to over-estimate the electron temperature in RF plasmas, since the operating power is low at only $130 \mathrm{~W}$ in this case the amount of RF noise is small enough that the effect is not large and can be neglected.

Measurements were made in the exhaust region of the HDLT between $\mathrm{z}=8 \mathrm{~cm}$ and $\mathrm{z}=18 \mathrm{~cm}$ for the standard operating conditions described above $(10 \mathrm{sccm}$, 


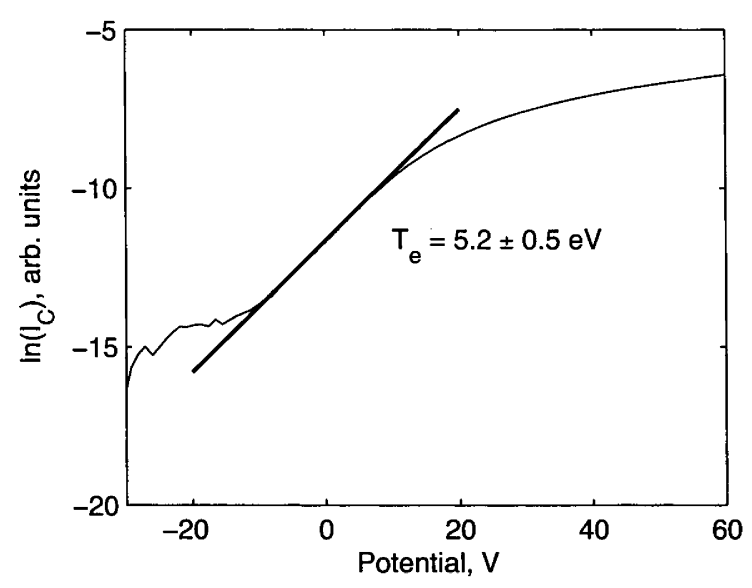

Figure 4.6: The electron temperature determined from the Langmuir probe I-V curve obtained downstream at $\mathrm{z}=18 \mathrm{~cm}$.

$0.4 \mathrm{mTorr}, 130 \mathrm{~W}$ and $138 \mathrm{G})$. Since the gradient of a linear fit of the natural logarithm of the electron current gives $T_{e}$ directly in volts, as described in Section 2.5.3, the resultant electron temperature across this region was found to be $5.2 \pm 0.5 \mathrm{eV}$. Figure 4.6 shows an example of the natural logarithm of the electron current and the appropriate linear fit. This result is in good agreement with other measurements of $T_{e}$ made downstream with a RF compensated probe in similar helicon systems (Takahashi et al., 2007).

Alternatively, the electron temperature can be deduced from the RFEA potential measurements and the Langmuir probe density measurements. Assuming a constant electron temperature along the axis of expansion, the axial density variation under the action of a spatially varying plasma potential can be determined from the Boltzmann relation,

$$
n_{1}=n_{2} \exp \left(\left(V_{p_{1}}-V_{p_{2}}\right) / T_{e}\right)
$$

where $n_{1}, V_{p_{1}}$ and $n_{2}, V_{p_{2}}$ are the plasma density and local plasma potential at two locations along the axis of expansion. This relation can also be used to demonstrate that there is a Boltzmann expansion downstream with a constant electron temperature (Chan et al., 1983; Charles et al., 1991, 1992). The electron temperature is found by rearranging Equation 4.2 such that

$$
T_{e}=\frac{V_{p_{1}}-V_{p_{2}}}{\ln \frac{n_{1}}{n_{2}}}
$$

Figure 4.7 shows the logarithmic plot of the measured plasma density versus the local measured plasma potential in the region downstream of the source (between $z=20 \mathrm{~cm}$ and $\mathrm{z}=83 \mathrm{~cm}$ ) for the standard operating conditions described 


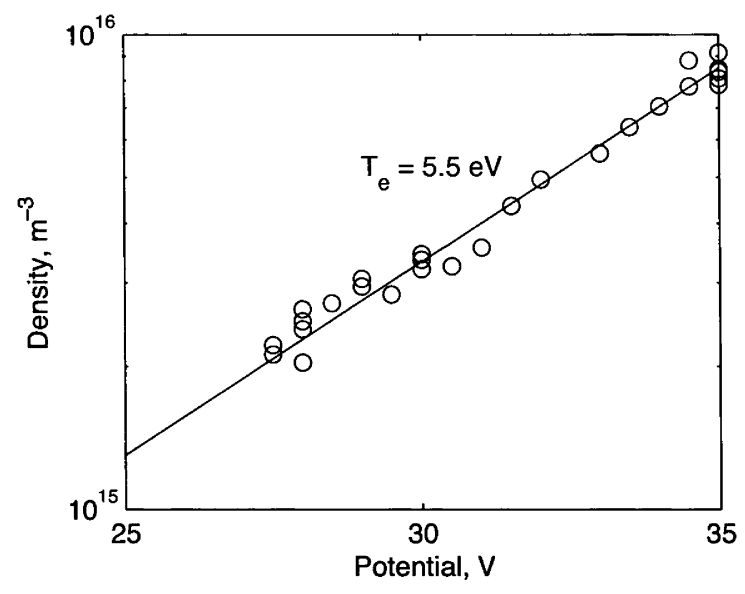

Figure 4.7: Plasma density, $n$, as function of local plasma potential $V_{p}$ downstream between $\mathrm{z}=20 \mathrm{~cm}$ and $\mathrm{z}=83 \mathrm{~cm}$.

above. The data in this region fits very well to a straight line, where the slope yields an electron temperature of $5.5 \mathrm{eV}$ (from Equation 4.3). This result is in good agreement with the temperature deduced directly from the Langmuir probe current-voltage characteristic described earlier, and suggests that downstream of the HDLT source the plasma follows a Boltzmann expansion (Aanesland and Charles, 2006). This result is also consistent with downstream measurements (5.5 eV) taken with a RF compensated probe in Chi Kung (Takahashi et al., 2007).

\subsection{Radial Plasma Characterisation}

One cause of inefficiency in the thrust produced by a propulsion system is divergence of the exhaust plume. The beam divergence, as it is known for electric propulsion systems, leads to a reduction in the component of the thrust along the thruster axis. Detailed radial measurements of the ion beam have been conducted in the Chi Kung experiment (Cox et al., 2008a) and have been used to determine the beam divergence for the HDLT. Although not the focus of this thesis, the average beam divergence in the Chi Kung experiment was found to be approximately $9^{\circ}$ and $98 \%$ of the momentum imparted to the ions in the beam was parallel to the thruster axis. Radial characterisation of the downstream plasma and ion beam produced by the HDLT prototype can also provide useful insights into the plasma behaviour and how the ion beam density and velocity vary radially. 


\subsubsection{Radial Plasma and Beam Potential Profile}

Using the radially installed RFEA, IEDF measurements were made as a function of radial position with the RFEA probe facing the HDLT source $\left(\theta=0^{\circ}\right)$ at $\mathrm{z}=9 \mathrm{~cm}$ and at $\mathrm{z}=15 \mathrm{~cm}$. Standard operating conditions were used $(10 \mathrm{sccm}$, 0.4 mTorr, $130 \mathrm{~W}$ and $138 \mathrm{G}$ ) and the normalised IEDFs are shown in Figure 4.8. The ion beam potential, $V_{b}$, is reasonably constant radially and the ion beam can be detected out to $\mathrm{r}=9 \mathrm{~cm}$ at $\mathrm{z}=9 \mathrm{~cm}$ and out to $\mathrm{r}=13 \mathrm{~cm}$ at $\mathrm{z}=15 \mathrm{~cm}$. This result is consistent with other measurements in similar devices (Charles et al., 2006) and strongly suggests that the double layer and the ion beam velocity are radially uniform for these operating conditions.

At $\mathrm{z}=9 \mathrm{~cm}$, the local plasma potential, $V_{p}$, is highest near $\mathrm{r}=0 \mathrm{~cm}$ and decreases radially, whereas at $\mathrm{z}=15 \mathrm{~cm}, V_{p}$ is constant radially in the region of the HDLT source $(\mathrm{r}<7.5 \mathrm{~cm})$ before beginning to decrease slowly as $\mathrm{r}>7.5 \mathrm{~cm}$. Beyond the region of the exhaust plume $(r>15 \mathrm{~cm})$, the background plasma has a similar potential profile at both $\mathrm{z}=9 \mathrm{~cm}$ and $\mathrm{z}=15 \mathrm{~cm}$ and decreases slowly as it approaches the wall of the space simulation chamber.

\subsubsection{Radial Ion Flux Profile}

The radial ion flux profile at $\mathrm{z}=9 \mathrm{~cm}$ and at $\mathrm{z}=15 \mathrm{~cm}$ was determined for the standard operating conditions from the total collector current, $I(0)$, measured with the RFEA. Since the RFEA is facing the HDLT source at $\theta=0^{\circ}, I(0)$ is a measurement of the total flux from both the ion beam and the local plasma. Figure 4.9 shows the profiles obtained at both locations and it is clear that the total flux is higher close to the centreline. This is an expression of the higher density inside the source (compare the axial density profile discussed earlier and shown in Figure 4.5). However, the radial ion flux is not uniform across the radius corresponding with the radius of the HDLT source tube $(\mathrm{r}<7.5 \mathrm{~cm})$ as expected. Beyond $\mathrm{r}=20 \mathrm{~cm}$ the radial ion fluxes at both axial locations are the same, consistent with a separate background plasma being present downstream distant from the HDLT source and the ion beam. Since no ion beam is present in this region, as shown earlier in Figure 4.8, the ion flux measurement is a qualitative measurement of the density of the background plasma.

For the measurements made at $\mathrm{z}=9 \mathrm{~cm}$, an interesting increase in the ion flux is observed between $r=7.5 \mathrm{~cm}$ and $\mathrm{r}=15 \mathrm{~cm}$ with a local maximum at approximately $\mathrm{r}=11 \mathrm{~cm}$. Radial density measurements with the Langmuir probe at this position confirmed the presence of this feature. This may be an expression of the 


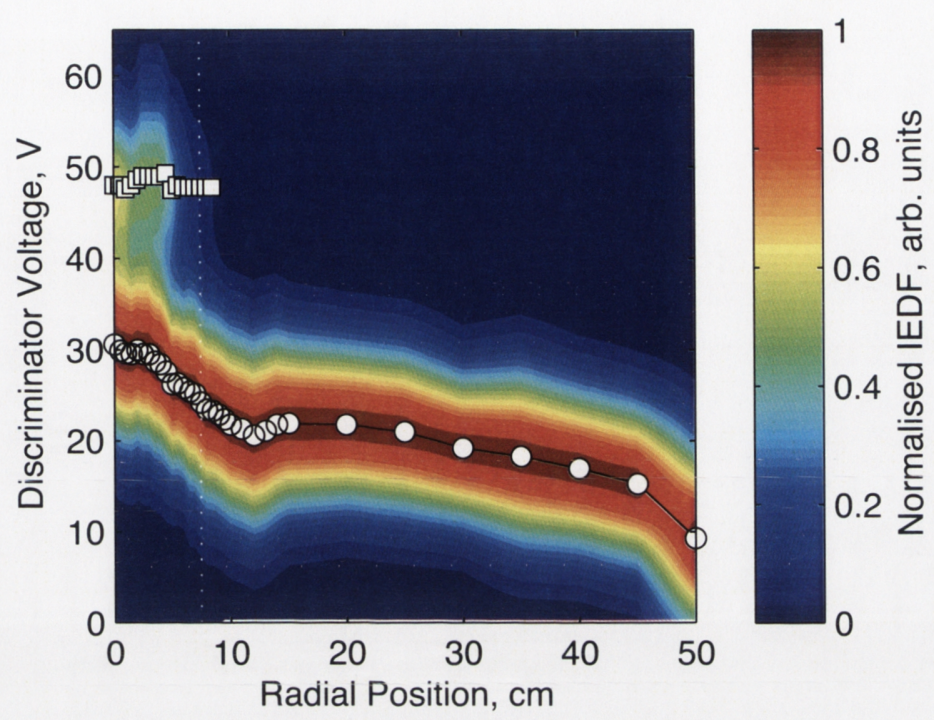

(a)

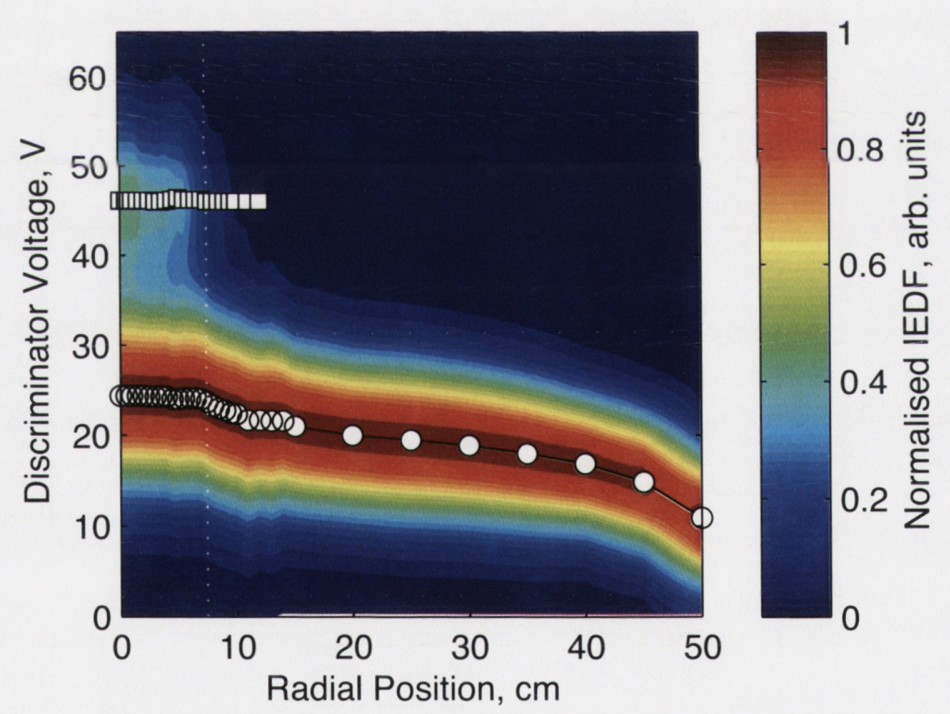

(b)

Figure 4.8: The radial profile of the IEDFs measured downstream with the RFEA (a) at $\mathrm{z}=9 \mathrm{~cm}$ and $(\mathrm{b})$ at $\mathrm{z}=15 \mathrm{~cm}$. The local plasma potential $(\bigcirc)$ and ion beam potential $(\square)$ are shown. The radial position corresponding to the edge of the source tube is marked with the dotted line. 


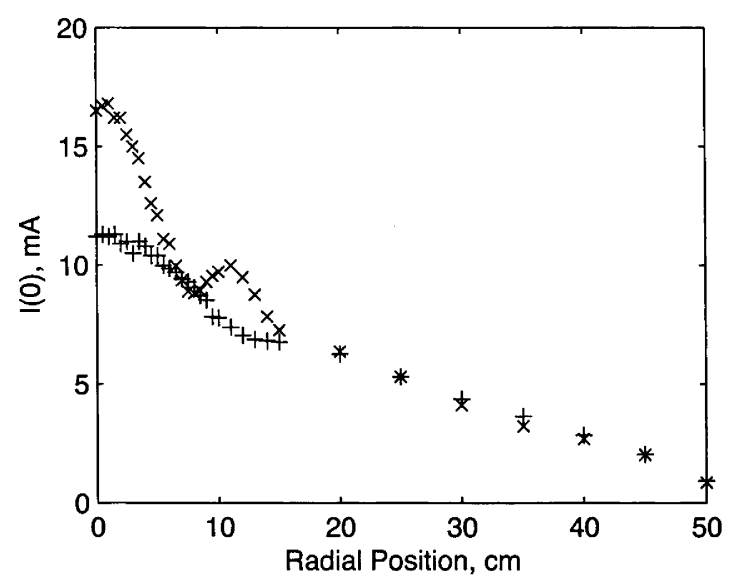

Figure 4.9: The radial ion flux profile measured with the RFEA downstream at $z=9 \mathrm{~cm}$ $(\times)$ and at $z=15 \mathrm{~cm}(+)$.

higher density annulus observed in the Chi Kung experiment when the downstream density was measured three-dimensionally by Cox et al. (2008b). Here, a higher density ring was found to surround the lower density core where the ion beam was measured. Takahashi et al. (2009) have measured a bi-Maxwellian distribution in this region and attributes the local ion density maximum in Chi Kung to the transport of electrons, with an energy higher than the ionisation energy (about $16 \mathrm{eV}$ ), from the source along the outermost magnetic field line that does not intersect the source wall. These energetic electrons may result in an increase in the local ionisation in this region and hence the increase in ion flux observed here between $\mathrm{r}=7.5 \mathrm{~cm}$ and $\mathrm{r}=15 \mathrm{~cm}$.

However in contrast to the Chi Kung measurements, in this case no low density core is observed in the central region just downstream of the HDLT prototype and the total ion flux measured is higher in the region where the ion beam is detected. This is likely a result of the larger volume diffusion chamber, with a radius of $50 \mathrm{~cm}$ compared to $15 \mathrm{~cm}$ for Chi Kung, and the subsequent expansion and diffusion of the plasma radially. Future measurements may yield further insights into this phenomenon and the relationship between the emerging magnetic field lines and the plasma density.

Figure 4.10 shows $I_{b}$, the current collected by the RFEA at the discriminator voltage that corresponds to the ion beam energy, as a function of radial position. Measurements were made downstream with the RFEA positioned at $z=9 \mathrm{~cm}$ and at $\mathrm{z}=15 \mathrm{~cm} . I_{b}$ is essentially a measure of the ion beam flux, $n_{b} v_{b}$. Since $V_{b}$ is reasonably constant radially, the beam velocity is assumed constant and therefore $I_{b}$ is a qualitative measurement of the ion beam density. As the signal 


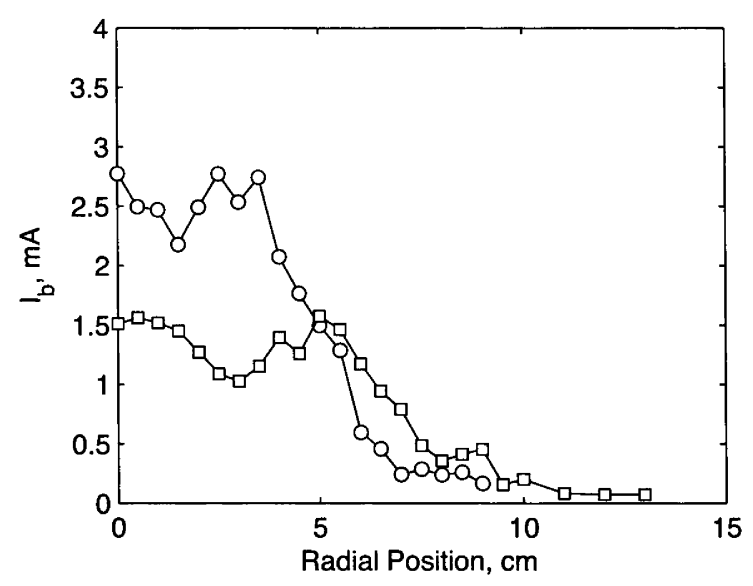

Figure 4.10: The radial ion beam flux profile measured with the RFEA downstream at $\mathrm{z}=9 \mathrm{~cm}(\square)$ and at $\mathrm{z}=15 \mathrm{~cm}(\bigcirc)$.

strength is quite low and the error large for these measurements, it is difficult to determine whether across the radius of the HDLT source tube $(\mathrm{r}<7.5 \mathrm{~cm}) I_{b}$ is constant or exhibits a 'double hump' radial density profile. The latter has been observed elsewhere and is indicative of an inductively coupled plasma in the source (Charles et al., 2006; Cox et al., 2008a).

However, it is likely that a 'double hump' ion beam profile is not observed here as measurements are made at only $130 \mathrm{~W}$ and the plasma is most likely operating in the capacitively coupled mode. Beyond the radius of the HDLT source tube $(\mathrm{r}>7.5 \mathrm{~cm})$, Figure 4.10 shows that the ion beam flux (and density) decreases rapidly which suggests that the majority of the ion beam flux is accelerated parallel to the thruster axis as found by Cox et al. (2008a).

\subsubsection{Radial Expansion and Electron Temperature}

Using the methodology outlined in Section 4.2.3, some interesting insights into the radial expansion of the plasma can be obtained and the electron temperature radially at different axial positions can also be determined. Figure 4.11 shows the radial ion flux determined from the RFEA measurements made earlier between $\mathrm{r}=0 \mathrm{~cm}$ and $\mathrm{r}=50 \mathrm{~cm}$ (see Section 4.3.2) as a function of the measured plasma potential, also between $r=0 \mathrm{~cm}$ and $r=50 \mathrm{~cm}$ (see Section 4.3.1). The results for two cases are presented; with the RFEA positioned at $\mathrm{z}=9 \mathrm{~cm}$ and with the RFEA positioned at $z=15 \mathrm{~cm}$. Using Equation 4.3, the temperature of the electron population was found to be about $6.3 \mathrm{eV}$ at $\mathrm{z}=9 \mathrm{~cm}$ (Figure 4.11a) and about $5.5 \mathrm{eV}$ at $z=15 \mathrm{~cm}$ (Figure 4.11b). As expected, the electron temperature is slightly higher 
when closer to the HDLT prototype but the results here are in good agreement with those determined earlier from axial measurements (Section 4.2.3) and from measurements in other similar experiments (Takahashi et al., 2009).

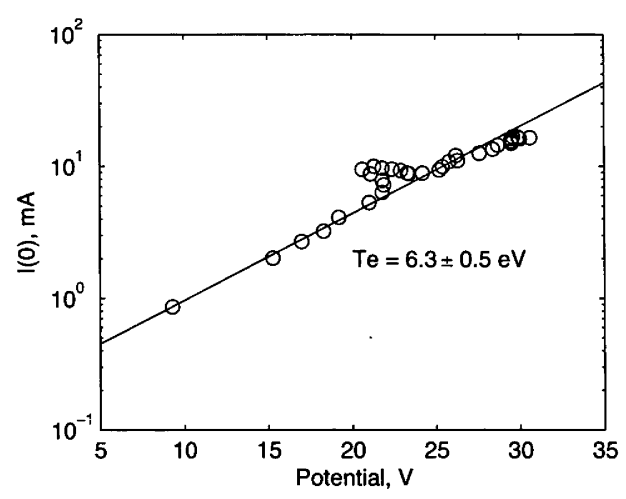

(a)

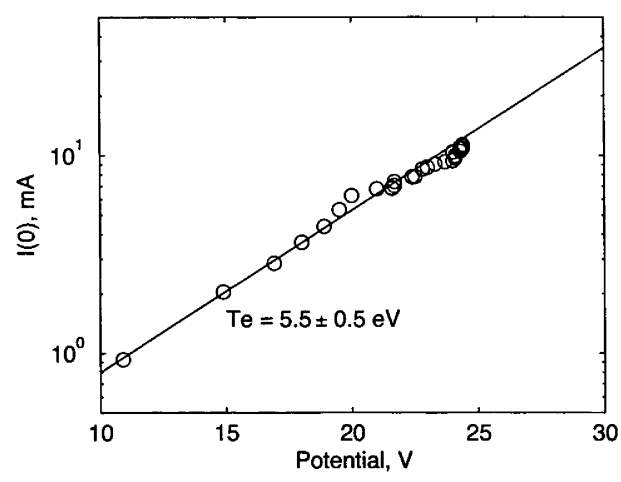

(b)

Figure 4.11: The radial total ion flux vs the plasma potential measured radially downstream with the RFEA (a) at $\mathrm{z}=9 \mathrm{~cm}$ and (b) at $\mathrm{z}=15 \mathrm{~cm}$.

For both cases, the trend line fits particularly well at the lower potentials, which corresponds to the plasma near the wall of the space simulation chamber $(\mathrm{r}>20 \mathrm{~cm})$. This suggests that the downstream plasma that is not part of the HDLT exhaust, i.e. the plasma in the region where no ion beam is detected and where the plasma from the source has little influence, follows a Boltzmann expansion radially with a constant electron temperature and is acting independently of the HDLT exhaust. The unusual feature in Figure 4.11a at a potential of $\sim 20 \mathrm{~V}$ is probably a result of the increase in the ion flux caused by the high density annulus discussed earlier in Section 4.3.2.

\subsection{Double Layer Characterisation}

Although in this configuration it is difficult to determine the precise location of the potential discontinuity associated with the double layer, it is clear from the IEDFs obtained, such as that in Figure 4.2b, that an ion beam is present downstream. From Figure 4.3 it is also clear that the double layer is located somewhere in the region between $\mathrm{z}=1 \mathrm{~cm}$ and $\mathrm{z}=5 \mathrm{~cm}$. This region is beyond the location of the magnetic field maximum $(\mathrm{z}=-5 \mathrm{~cm})$ but in the region of the maximum of the magnetic field gradient $(\mathrm{z}=2 \mathrm{~cm})$. In measurements made in WOMBAT, Chi Kung and another helicon reactors, the position of the double layer is in the 
vicinity of the location of the maximum of the magnetic field gradient (Charles, 2007).

The potential drop of the double layer, $V_{D L}$, which equals $V_{b}-V_{p}$, was found to be $16 \mathrm{~V}$ when measured with the RFEA probe $10 \mathrm{~cm}$ from the exit of the HDLT source $(z=13 \mathrm{~cm})$. This position was chosen for the comparison because past measurements of the potential drop of the double layer in the Chi Kung experiment have been made at a similar distance from the exit of the source tube. This result is very similar to that measured in the Chi Kung experiment, on which the HDLT is based, at 0.4 mTorr in argon (Charles, 2005a) and that observed in other recent investigations (Plihon et al., 2007). The velocity of the ions in the beam, which are accelerated by the potential drop $V_{D L}$, can be calculated using

$$
v_{b_{D L}}=\sqrt{\frac{2 e\left(V_{b}-V_{p}\right)}{m_{i}}}=\sqrt{\frac{2 e V_{D L}}{m_{i}}}
$$

where $e$ is the electron charge and $m_{i}$ is the argon ion mass. In this case, where the pressure is $0.4 \mathrm{~m}$ Torr and $V_{D L}=16 \mathrm{~V}, v_{b_{D L}}$ equals $\sim 8.7 \mathrm{kms}^{-1}$.

Meige et al. (2005b) found that extending the location of the grounded right wall downstream to $50 \mathrm{~cm}$ from the double layer in a PIC simulation did not influence the potential profile or significantly alter the source plasma potential or the potential drop of the double layer. In this configuration where the grounded wall is $80 \mathrm{~cm}$ distant, no significant changes are observed either. This result suggests that a current-free double layer and ion beam can be formed when the grounded wall is removed completely and the expansion region is infinite, as would be the case when the HDLT is operated in space.

\subsection{Pressure Dependence}

The potential drop of the double layer has been shown to be a function of the operating pressure (Charles, 2005a; Takahashi et al., 2007; Plihon et al., 2007; Charles, 2007; Byhring et al., 2008) and a theoretical model has been developed for the formation of a low-pressure current-free double layer inside an upstream insulating source chamber connected to a larger diameter vacuum chamber downstream (Lieberman et al., 2006). With the RFEA positioned $10 \mathrm{~cm}$ from the exit of the HDLT source tube (at $z=13 \mathrm{~cm}$ ), an experimental study of $V_{D L}$ versus pressure was undertaken. The results are shown in Figure 4.12. For comparison, the experimental data for the Chi Kung experiment and the theoretical model for argon, as detailed by Lieberman et al. (2006), are also shown. 


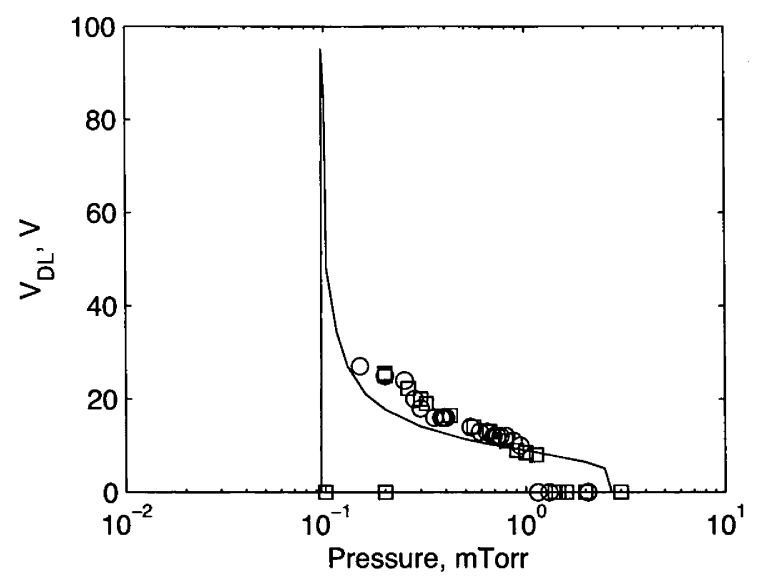

Figure 4.12: Double layer strength, $V_{D L}$, versus pressure: HDLT immersed in space simulation vacuum chamber $(O)$; results for HDLT attached to Chi Kung vacuum chamber $(\square)$ and theory (-) are from Lieberman et al. (2006).

Figure 4.12 shows that the double layer in this experiment is present over a distinct pressure range, disappearing below the low pressure threshold of 0.15 mTorr and above the high pressure threshold of 1 mTorr. Within this pressure range, $V_{D L}$ increases rapidly as the pressure is decreased. This is consistent with the increase in the source potential observed by Charles (2005a) when decreasing the operating pressure in the Chi Kung experiment.

The theoretical model developed by Lieberman et al. (2006) predicts that the double layer disappears above pressures of a few mTorr. Other recent studies by Plihon et al. (2007) and Byhring et al. (2008) have also observed this upper pressure threshold for double layer formation. In this case, with the HDLT prototype immersed inside the vacuum chamber, the low pressure threshold is slightly lower than that observed for the Chi Kung experiment. A lower pressure threshold was also observed for the double layer found in the WOMBAT experiment (Sutherland et al., 2005), which has a larger downstream diffusion chamber than the Chi Kung experiment. In the experimental apparatus under investigation in this thesis, at operating pressures below $0.15 \mathrm{~m}$ Torr, the propellant flow rate is very low, less than $3 \mathrm{sccm}$, and so it is not possible to produce a plasma discharge at the RF power used here $(130 \mathrm{~W})$.

It is acknowledged that this operating pressure $(0.15 \mathrm{mTorr})$ is higher than the operating pressures on-orbit and so studies in a space simulation vacuum chamber where high propellant flow rates are possible while maintaining operating pressures less than $5 \times 10^{-6}$ Torr, would be beneficial. It is, however, worth noting that Charles et al. (2006) have measured a current-free double layer in the 
Chi Kung experiment using xenon at a pressure of 0.07 mTorr. For larger geometries with inherent and known axial pressure gradients such as the MNX device, which is capable of maintaining pressures in the downstream vacuum chamber below $1.5 \times 10^{-5}$ Torr during operation, Cohen et al. (2003) and Sun et al. (2005a) also report the formation of current-free double layers.

The agreement between the results obtained here with the HDLT prototype immersed in a space simulation chamber and previous experiments suggests that the properties of the double layer and ion beam are consistent with the currentfree double layers observed previously in experiments with the helicon source attached to a downstream vacuum chamber. Therefore, the results from these previous studies may be applied directly to the optimisation of the HDLT for space applications. Furthermore, at 0.15 mTorr, $V_{D L}=27 \mathrm{~V}$ which results, using Equation 4.4 , in a $v_{b_{D L}}$ of approximately $11.4 \mathrm{kms}^{-1}$. This increase in the ion beam velocity at lower pressures translates into an increase in the specific impulse and demonstrates that the HDLT specific impulse is scalable, an attractive feature for long-duration missions where varying levels of thrust and specific impulses may be required.

\subsection{Magnetic Field Dependence}

The magnetic field topography and magnitude are important parameters that influence the formation of a current-free double layer (Charles, 2005a; Plihon et al., 2007; Charles and Boswell, 2008; Biloiu et al., 2008) and hence the ion beam created by the HDLT prototype. Charles et al. (2008a) have also recently demonstrated that the ion beam produced by the HDLT can be magnetically steered with the addition of a transverse solenoid. Measurements in Chi Kung have shown that the presence and strength of the double layer strongly depend on the magnetic field and that when no axial magnetic field is applied, no double layer is observed. When sufficient axial magnetic field $(\sim 50-70 \mathrm{G})$ is applied, the formation of the double layer can be triggered as the density gradient at the end of the plasma source increases (Charles and Boswell, 2007). It was found that after this transition, an ion beam is observed downstream.

To investigate the magnetic field dependence, measurements were made using the radially installed RFEA positioned at $z=9 \mathrm{~cm}$, on the centreline at $r=0 \mathrm{~cm}$ and facing the HDLT source at $\theta=0^{\circ}$ when operating the HDLT prototype at standard conditions (10 sccm, 0.4 mTorr and $130 \mathrm{~W}$ ) and varying the current applied to both solenoids. The potential of the ion beam and the local downstream 


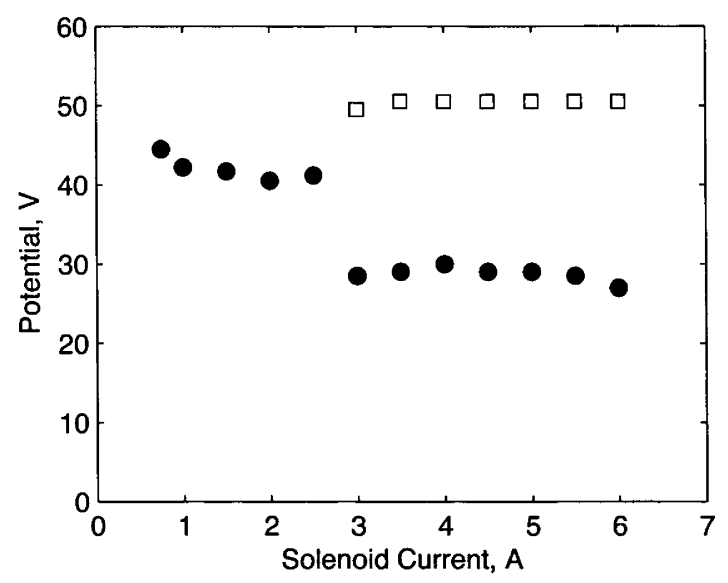

Figure 4.13: The potential of the ion beam $(\square)$ and the local plasma $(\bullet)$ versus solenoid current measured with the RFEA at $z=9 \mathrm{~cm}, r=0 \mathrm{~cm}$. At $3 \mathrm{~A}$ the maximum axial dc magnetic field, $B_{z}=138 \mathrm{G}$.

plasma are shown in Figure 4.13 as a function of the solenoid current. These measurements reveal that an ion beam is only present when $3 \mathrm{~A}$ or greater, that is $138 \mathrm{G}$, is applied to the solenoids (for equally applied currents). Below $3 \mathrm{~A}$ only one peak is present in the IEDF produced by the RFEA indicating that no ion beam is formed and that an expanding plasma results. In Figure 4.13, it is clear that when an ion beam is present, $V_{b}$ is constant with increasing solenoid current and therefore the ion beam velocity is constant. These results are in good agreement with those observed in Chi Kung and other experiments (Charles, 2005a; Charles and Boswell, 2007; Byhring et al., 2008; Biloiu et al., 2008). The total current collected by the RFEA, that is the total plasma flux, was also observed to increase with increasing solenoid current which indicates that the plasma density and hence thrust can be scaled up if desired.

Radial profiles of the plasma potential and total ion flux were also obtained with the RFEA positioned at $\mathrm{z}=15 \mathrm{~cm}$ and facing the HDLT source at $\theta=0^{\circ}$. Two cases were investigated: operating the HDLT prototype at standard conditions where $B_{z}=138 \mathrm{G}$ and with no magnetic field at 0.4 mTorr and at $130 \mathrm{~W}$ of RF power. The radial potential and ion flux profiles are shown in Figures 4.14 and 4.15 . The interpretation of results for the standard conditions are presented earlier in Section 4.3 and will not be repeated here.

When operating with no magnetic field, no ion beam is detected and the plasma potential measured downstream across the radius of the HDLT source is significantly higher $(\sim 43 \mathrm{~V})$ compared to when operating at standard conditions $(\sim 25 \mathrm{~V})$. The potential of the background plasma near the walls of the space sim- 


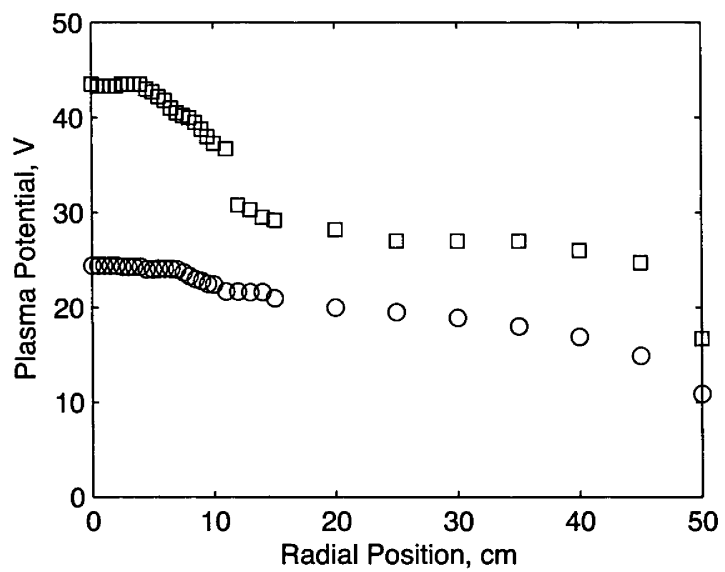

Figure 4.14: The radial potential profiles at the standard magnetic field configuration where $B_{z}=138 \mathrm{G}(\bigcirc)$ and with no magnetic field ( $\square$ ).

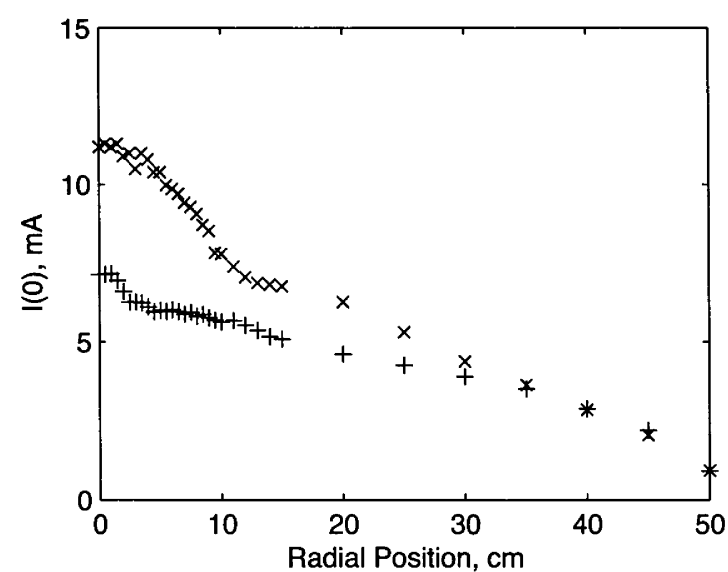

Figure 4.15: The radial ion flux profiles at the standard magnetic field configuration where $B_{z}=138 G(\times)$ and with no magnetic field $(+)$. 
ulation chamber $(\mathrm{r}>20 \mathrm{~cm})$ is also about $5 \mathrm{~V}$ higher than that measured for the standard conditions. The total ion flux measured with the RFEA for the no magnetic field case, however, is much lower than that measured for the standard conditions, suggesting that the plasma density is much lower downstream. These results are consistent with earlier experiments that have shown that when no magnetic field is present, no ion beam is detected and the plasma density downstream is low (Charles, 2005a; Charles and Boswell, 2007; Plihon et al., 2007).

\subsection{Effect of Varying Physical Configuration}

To investigate the effect of the position of the source tube, relative to the magnetic field, on the properties of the ion beam and double layer, the HDLT source tube was retracted by $3 \mathrm{~cm}$. Hence, the source tube was moved relative to both the HDLT structure and the antenna such that the open end of the source was in line with the HDLT structure at $\mathrm{z}=0 \mathrm{~cm}$. For the standard conditions described above ( $10 \mathrm{sccm}, 0.4 \mathrm{mTorr}, 130 \mathrm{~W}$ and $138 \mathrm{G}$ ) the RFEA was used to measure $V_{D L}$, the potential drop of the double layer, at a position $13 \mathrm{~cm}$ from the end of the HDLT structure $(z=13 \mathrm{~cm})$. This position is $11 \mathrm{~cm}$ from the maximum gradient of the magnetic field. Prior to retracting the HDLT source $V_{D L}=16 \mathrm{~V}$, as outlined earlier in Section 4.4 , and with the source tube retracted $3 \mathrm{~cm} V_{D L}=15.5 \mathrm{~V}$. Hence no appreciable difference in the potential drop of the double layer, and hence the acceleration received by the ions, results from moving the HDLT source tube relative to the magnetic field and the antenna.

The density was also measured as a function of axial position with the HDLT source tube retracted $3 \mathrm{~cm}$. This allowed the effect of moving the source tube relative to the magnetic field and antenna on the density profile to be investigated. Measurements were made between $\mathrm{z}=-10 \mathrm{~cm}$ and $\mathrm{z}=15 \mathrm{~cm}$ for the same conditions described above using the RFEA. Figure 4.16 shows the density profile obtained with the HDLT source tube retracted by $3 \mathrm{~cm}(x)$. When compared to the density profile obtained when the source tube is extended $3 \mathrm{~cm}$ beyond the HDLT structure ( $\bigcirc$ in Figure 4.16), it is clear that the density profile does not change as a result of moving the source tube relative to the magnetic field and the HDLT structure and antenna.

These two results show that moving the source tube does not adversely affect the formation of the ion beam or the measured axial density profiles. Sutherland et al. (2005) have shown in the WOMBAT experiment that the double layer and the resultant ion beam track with the moving magnetic field structure and that 


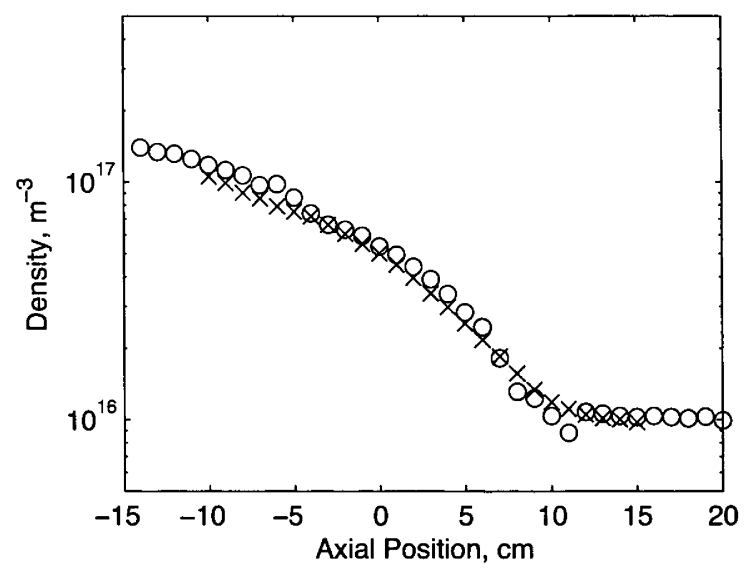

Figure 4.16: Plasma density, $n$, as function of axial position; standard conditions $(\bigcirc)$ and with HDLT source tube retracted $3 \mathrm{~cm}(\times)$.

the double layer position is independent of the plasma source or the physically expanding geometry of the experiment. The results obtained here confirm that the double layer is more dependent upon the magnetic field configuration than the physical configuration of the source and the downstream region consistent with the observations of Sutherland et al. (2005).

\subsection{Ion Beam Flux and Plasma Detachment}

Plasma detachment is an active area of research that is applicable to a variety of new magnetoplasma electric propulsion systems (Hooper, 1993; Arefiev and Breizman, 2005; Breizman et al., 2008; Schmit and Fisch, 2009). Recently it has been demonstrated experimentally by Deline et al. (2009) that a sufficiently energetic and flowing plasma experiences little measurable change in plume trajectory even in the presence of dramatic changes in magnetic field profile, suggesting that the plasma plume does detach. Despite not being the focus of this thesis, some conclusions can be made about the detachment of ions from the HDLT exhaust from the measurements made above.

Figure 4.17 shows the ion beam flux, $I_{b}$, versus the axial position downstream of the HDLT source obtained from the RFEA measurements described earlier in Section 4.2.1. The gyro radius for both electrons and ions is also included on a logarithmic scale for the standard magnetic field conditions present downstream. 


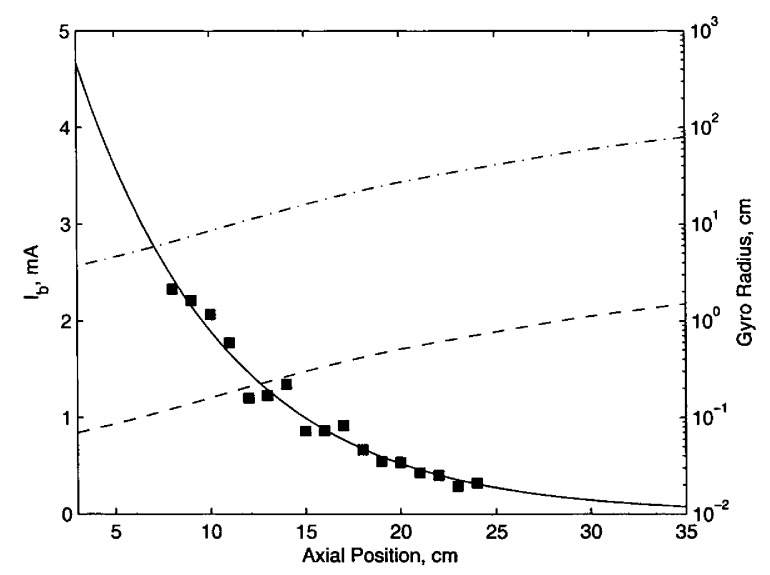

Figure 4.17: The ion beam flux, $I_{b}$, as function of axial position downstream of the HDLT source ( $\square$ ). An exponential line of best fit is shown (-). The gyro radius for ions (--) and electrons $(-\bullet)$ under the standard magnetic field conditions are also shown.

The gyro radius for electrons, $r_{g e}$, is found using

$$
r_{g e}=\frac{v_{T_{e}}}{\omega_{c e}}=2.38 \frac{\sqrt{T_{e}}}{B_{z}}
$$

where $v_{T_{e}}$ is the velocity of the electrons at a particular electron temperature, $\omega_{c e}$ is the gyrofrequency of the electrons, $T_{e}$ is the electron temperature in $\mathrm{eV}$ which is taken from the previously measured value of $5.2 \mathrm{eV}$ and $B_{z}$ is the axial magnetic field in Gauss. The gyro radius for ions, $r_{g i}$, is determined using

$$
r_{g i}=\frac{v_{T_{i}}}{\omega_{c i}}=102 \frac{\sqrt{\mu}}{\mathrm{Z}} \frac{\sqrt{T_{i}}}{B_{z}}
$$

where $v_{T_{i}}$ is the velocity of the ions at a particular ion temperature, $\omega_{c i}$ is the gyrofrequency of the ions, $\mu$ is the ratio of the ion mass to proton mass which for argon is $40, \mathrm{Z}$ is the charge state which for $\mathrm{Ar}^{+}$is $1, T_{i}$ is the ion temperature in $\mathrm{eV}$ which is assumed to be $0.2 \mathrm{eV}$ and $B_{z}$ is the axial magnetic field in Gauss.

It is clear from Figure 4.17 that $I_{b}$ decreases exponentially downstream with an $e$ folding of about $8 \mathrm{~cm}$. This decrease in the ion beam flux is the result of ionneutral charge-exchange collisions as discussed earlier in Section 4.2.1 and is expected. This result is also in good agreement with measurements made in a helicon system with permanent magnets (Takahashi and Fujiwara, 2009) and measurements made of the ion beam in Chi Kung with an RFEA (Lieberman et al., 2006) and with laser induced fluorescence (LIF). In the latter, the LIF intensity of the ion beam population was also shown to decay exponentially downstream (Keese et al., 2005). 
When the ion beam is first discernible at $\mathrm{z}=8 \mathrm{~cm}$ the electron gyro radius is $0.18 \mathrm{~cm}$ and the ion gyro radius is $8 \mathrm{~cm}$. At $\mathrm{z}=24 \mathrm{~cm}$, where the ion beam is still detectable, the electron gyro radius is $0.6 \mathrm{~cm}$ and the ion gyro radius is $40 \mathrm{~cm}$. At the back wall of the space simulation vacuum chamber $(z=83 \mathrm{~cm})$ the electron and ion gyro radius are $10 \mathrm{~cm}$ and $500 \mathrm{~cm}$, respectively. For reference, the radius of the HDLT source and the space simulation vacuum chamber are $7.5 \mathrm{~cm}$ and $50 \mathrm{~cm}$, respectively.

Analysis of the trajectories of exhaust ions from the HDLT by Gesto et al. (2006) has shown that since acceleration by the double layer is normal to the magnetic field and the magnetic field is reasonably low (maximum of $138 \mathrm{G}$ in this case), the gyromotion of the beam ions is very low and their velocity appears to remain axial. Also, in this experiment the values of the gyro radius approach the scales of the experiment in the region where the ion beam is detected which suggests that the ion beam is not magnetised and is no longer under the influence of the magnetic field. Spatially-resolved measurements of the $\mathrm{Ar}^{+}$and $\mathrm{Xe}^{+}$beam in the Chi Kung experiment have reached a similar conclusion (Charles, 2005b; Charles et al., 2006; Gesto et al., 2008; Cox et al., 2008a). When coupled with the work of Gesto et al. (2006), the experimental results presented here suggest that the ion beam formed by the HDLT prototype does detach from the magnetic field lines and will produce thrust when operating in space. Further work is required to confirm this conclusion.

\subsection{MFMI Measurements Downstream}

Using the MFMI described in Chapter 3, measurements were made of the force imparted by the thruster exhaust for various operating conditions. For these measurements, the $150 \mathrm{~mm}$ diameter target plate for the MFMI was used and it was positioned downstream at $\mathrm{z}=13 \mathrm{~cm}$, which is approximately one mean free path for ion-neutral charge exchange collisions (at 0.4 mTorr). Prior to undertaking such measurements, it is important to confirm that the presence of the target plate downstream does not influence the plasma and ion beam formed by the HDLT prototype. Therefore measurements were made with the radially installed RFEA positioned in front of the MFMI target plate as shown in Figure 4.18a with the orifice facing the HDLT exhaust $\left(\theta=0^{\circ}\right)$.

Figure $4.18 \mathrm{~b}$ shows the normalised $I_{c}$ vs $V_{d}$ characteristic and IEDF obtained on the centreline of the HDLT source $(\mathrm{r}=0 \mathrm{~cm})$ when operating at the standard conditions ( $10 \mathrm{sccm}, 0.4 \mathrm{mTorr}, 130 \mathrm{~W}$ and $138 \mathrm{G}$ ). The IEDF in Figure $4.18 \mathrm{~b}$ shows 


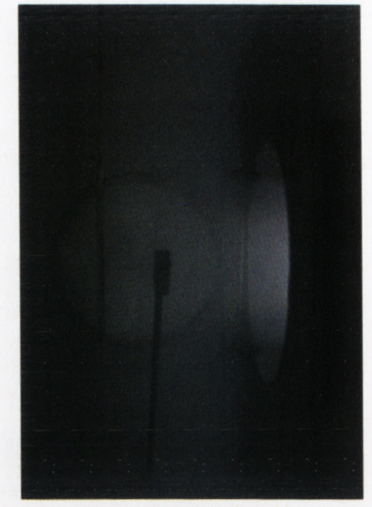

(a)

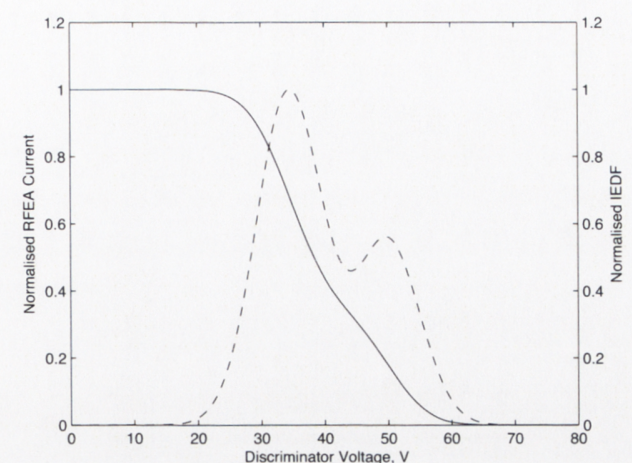

(b)

Figure 4.18: (a) The radially installed RFEA mounted in front of the MFMI target at $\mathrm{z}=9 \mathrm{~cm}, \mathrm{r}=0 \mathrm{~cm}, \theta=0^{\circ}$. The MFMI target is installed at $\mathrm{z}=13 \mathrm{~cm}$. (b) The normalised RFEA current (-) and the normalised IEDF (--) versus discriminator voltage measured with the RFEA. The plasma conditions are $130 \mathrm{~W}, 10 \mathrm{sccm}, 0.4 \mathrm{mTorr}$ and $138 \mathrm{G}$.

a clear ion beam, represented by the second peak of the IEDF, which is centred on $50 \mathrm{~V}$, the beam potential $V_{b} . V_{p}$, the local downstream plasma potential, equals $34.5 \mathrm{~V}$. These values for $V_{p}$ and $V_{b}$ are in agreement with the measurements made previously at this position with the RFEA facing the HDLT source under the same operating conditions described in Section 4.1 and confirm that the presence of the MFMI does not affect the formation of the ion beam by the HDLT prototype.

For these operating conditions, $V_{b}$ was found to equal $50 \mathrm{~V}$ and $V_{p}$ was found to equal $34.5 \mathrm{~V}$. Here $V_{b}$ varies by less than $5 \%$ across the radius of the target plate and is therefore assumed constant at $50 \mathrm{~V}$. Using Equation 3.21, the velocity at which the beam ions impact the MFMI target can be computed and was found to be $15,500 \mathrm{~ms}^{-1}$. The ions in the local plasma that enter the sheath on both the front and back sides of the MFMI target are accelerated and strike the target at the Bohm velocity. Using Equation 2.2 and the $T_{e}$ measured downstream earlier in Section $4.2 .3(5.2 \mathrm{eV}), v_{B}=3,530 \mathrm{~ms}^{-1}$. A non-uniform $T_{e}$ may affect $v_{B}$ as a function of radius. However, since the affect on the Bohm velocity is a $\sqrt{T_{e}}, v_{B}$ is presently assumed to be constant. The measurement with the RFEA in front of the MFMI target can also be used to determine what percentage of the ion population at the MFMI position is from the local plasma and from the ion beam. Using Equation 2.6, the ratio of the ion beam density to the local plasma density, $\frac{n_{b}}{n_{d s}} \approx 0.1 . \frac{n_{b}}{n_{d s}^{+}}$is also constant as a function of radius.

With the Langmuir probe positioned in front of the the MFMI target at $\mathrm{z}=12 \mathrm{~cm}$, the total density of the plasma in front of the target was measured and found to 
be about $2.6 \times 10^{15} \mathrm{~m}^{-3}$. Since $\frac{n_{b}}{n_{d s}} \approx 0.1$, the ion beam density at this position is $\sim 2.6 \times 10^{14} \mathrm{~m}^{-3}$ and $n_{d s}^{+}$is about $2.3 \times 10^{15} \mathrm{~m}^{-3}$. The density measured behind the MFMI target, with the Langmuir probe $1 \mathrm{~cm}$ behind the target plate at $\mathrm{z}=14 \mathrm{~cm}$, for these conditions is $1.98 \times 10^{15} \mathrm{~m}^{-3}$. The density difference between the front and back of the target in this case is about $15 \%$, which suggests that a wake is unlikely to be present. Visually, the plasma appears to be similar on both sides of the target, which suggests that on the first approximation the effect of the wake, if present at all, on $T_{e}$ and hence the Bohm velocity is not significant. Radial measurements with the Langmuir probe in front and behind the target plate show that for the conditions investigated here, the plasma density is constant from $\mathrm{r}=0 \mathrm{~cm}$ to $\mathrm{r}=6.5 \mathrm{~cm}$ and drops off sharply at $\mathrm{r}>6.5 \mathrm{~cm}$. $A_{p}$ is therefore calculated assuming a radius of $6.5 \mathrm{~cm}$.

Using the information obtained from the diagnostic probes outlined above, the force on the target from the downstream plasma and the ion beam can be calculated. $F_{d s}^{+}$and $F_{d s}^{-}$, the forces on the front and back of the target from the downstream plasma were found to be similar at $25.37 \mu \mathrm{N}$ and $21.89 \mu \mathrm{N}$, respectively. In this case, the target was about one mean free path from the exit of the HDLT source which ensured a very low ion beam density and force comparable to that from the neutrals. Using Equation 3.20 and the measurements with the diagnostic probes, the calculated force from the ion beam was found to be $54.22 \mu \mathrm{N}$. Since $F_{p}=F_{b}+F_{d s}^{+}-F_{d s}^{-}$the calculated force from the plasma, $F_{p}=57.70 \mu \mathrm{N}$.

For these operating conditions the displacement of the reflected laser beam spot of the MFMI was measured and found to be $\Delta y=111.1 \pm 14.1$ units with the propellant flow alone and $\Delta y=214.4 \pm 17.5$ units with the HDLT operating and the plasma ignited. Using the calibration curve for the $150 \mathrm{~mm}$ diameter target in Figure 3.7a the force measured on the target with the propellant flow alone, $F_{n}$, is $66.0 \pm 12.2 \mu \mathrm{N}$ and with the HDLT operating and the plasma ignited, $F_{t}=123.3 \pm 14.2 \mu \mathrm{N}$. Therefore the force from the plasma, $F_{p}$, measured by the MFMI is approximately $57.3 \mu \mathrm{N}$ which is in good agreement with the value of $F_{p}$ calculated from the information obtained from the measurements made with the diagnostic probes.

\subsubsection{Varying Propellant Flow Rate}

Measurements were made at various flow rates with the HDLT operating at $130 \mathrm{~W}$ of RF power and a maximum axial dc magnetic field inside the HDLT source of $138 \mathrm{G}$. The results are shown in Figure 4.19 along with the calculated force from the neutrals, $F_{n}$, plotted as a solid line. For reference, here and in the 


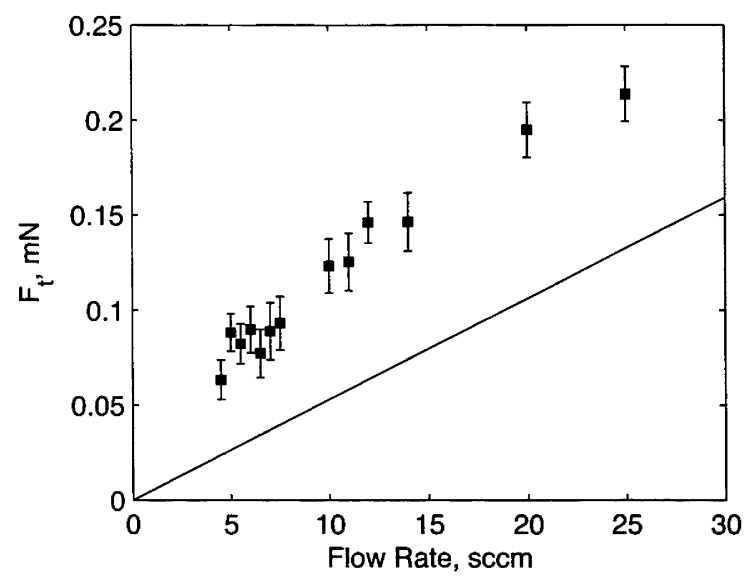

Figure 4.19: Force on the MFMI target for a plasma formed at $130 \mathrm{~W}$ and $138 \mathrm{G}$ at varying flow rates ( $)$. The calculated force from the neutrals, $F_{n}$, is also shown (-).

subsequent figures in this section, the results are plotted versus the flow rate for clarity and consistency with the results shown in Section 3.7. For these experiments, the flow rate scales linearly at the same rate as the operating pressure so that a flow rate of $10 \mathrm{sccm}$ corresponds to an operating pressure of $0.4 \mathrm{mTorr}$. The flow rate range investigated $(0-25 \mathrm{sccm})$ corresponds to a pressure range of $0-1.1$ mTorr.

The plasma parameters were also investigated under these conditions using the diagnostic probes positioned at $\mathrm{r}=0 \mathrm{~cm}$ and while varying the propellant flow rate between $5 \mathrm{sccm}$ and $13.9 \mathrm{scmm}$. The RFEA was positioned in front of the MFMI target at $\mathrm{z}=9 \mathrm{~cm}$ with the orifice facing the HDLT exhaust $\left(\theta=0^{\circ}\right)$ and the Langmuir probe was positioned $1 \mathrm{~cm}$ in front of the MFMI target $(\mathrm{z}=12 \mathrm{~cm})$ and $1 \mathrm{~cm}$ behind the MFMI target $(\mathrm{z}=14 \mathrm{~cm})$. From the RFEA measurements shown as $\square$ in Figure 4.20 it is clear that the ion beam energy increases as the propellant flow rate is decreased and therefore, as per Equation 3.21, the ion beam velocity also increases with decreasing flow rate. These results are consistent with the results discussed in Section 4.5.

Using the RFEA, the ratio of the ion beam density to the local downstream plasma density, $\frac{n_{b}}{n_{d s}}$, calculated from Equation 2.6, was determined and found to remain reasonably constant at 0.1 as the flow rate is varied. From the Langmuir probe measurements, the total density in front and behind the MFMI target increases quasi linearly with increasing flow rate as shown in Figure 4.20. Since $\frac{n_{b}}{n_{d s}}$ remains constant over this pressure range, increasing the flow rate also increases $n_{b}$ and hence $F_{b}$ from Equation 3.20.

Figure 4.21 shows the force from the plasma, $F_{p}$, measured with the MFMI ( $\square$ ) 


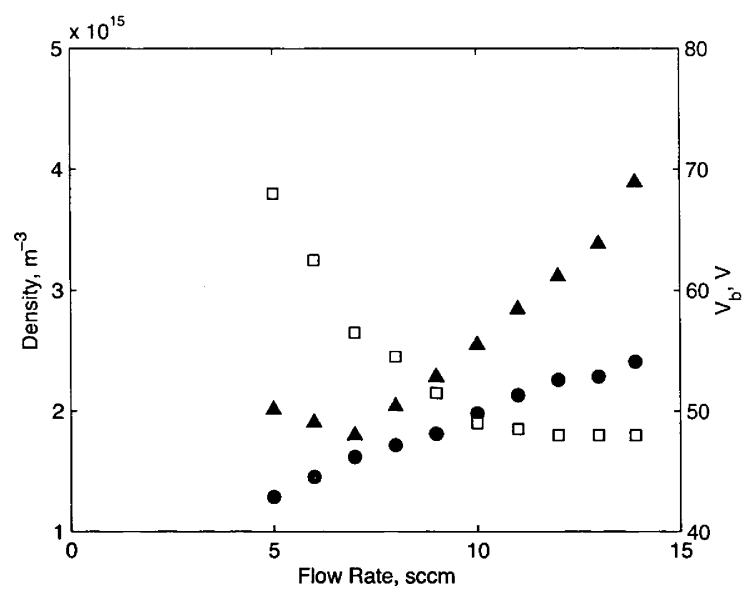

Figure 4.20: The total plasma density measured with the Langmuir probe positioned in front of the MFMI target at $\mathrm{z}=12 \mathrm{~cm}(\boldsymbol{\Lambda})$ and behind the MFMI target at $\mathrm{z}=14 \mathrm{~cm}(\bullet)$ as a function of flow rate. The beam potential, $\mathrm{V}_{b}$, versus flow rate as measured with the RFEA at $z=9 \mathrm{~cm}(\square)$ is also shown.

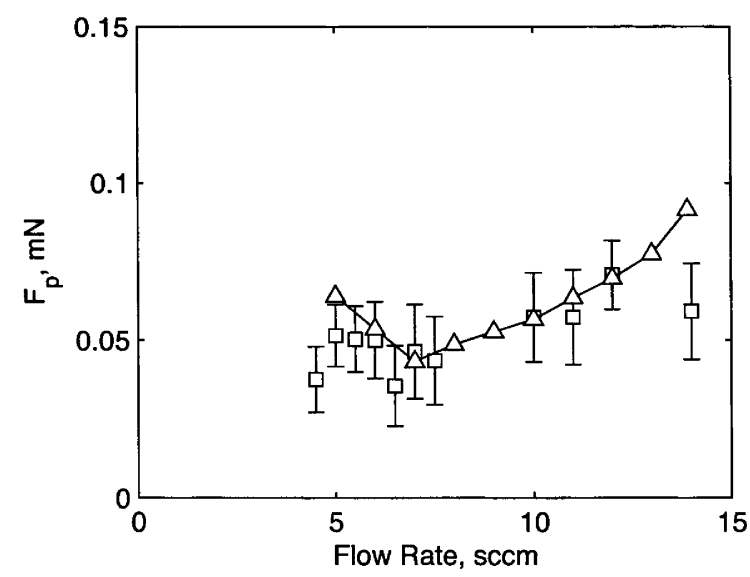

Figure 4.21: The force from the plasma, $F_{p}$, measured with the MFMI as a function of flow rate $(\square)$. The force from the plasma, calculated using Equation 3.22 and the information obtained from the diagnostic probes, as a function of flow rate $(\triangle)$. The solid line joining the data points is for clarity only. 
and calculated from Equation 3.22 using the measurements from the diagnostic probes $(\triangle)$ both as a function of flow rate. $v_{b}$ is calculated using $V_{b}$ measured with the RFEA and Equation 3.21 and $T_{e}$ is assumed invariant with flow rate over this range, therefore $v_{B}$ is constant at $3,530 \mathrm{~ms}^{-1}$. The measurements obtained with the MFMI are in good agreement with those calculated using the information from the measured plasma parameters. These measurements demonstrate that variations in momentum flux and hence force from different ion beam velocities and plasma densities can be characterised with this instrument.

\subsubsection{Varying Magnetic Field Strength}

As discussed in Section 4.6, the magnetic field topography and magnitude are important parameters that influence the formation of a current-free double layer (Charles and Boswell, 2003; Biloiu et al., 2008) and hence the ion beam created by the HDLT prototype. Recent work by Charles and Boswell (2007) has shown that increasing the maximum axial dc magnetic field also increases the plasma density inside the source. To investigate the effect of the magnetic field upon the force measured by the MFMI, the current applied to both solenoids, and hence the magnitude of the magnetic field, was varied when operating at $10 \mathrm{scmm}$ flow rate, $0.4 \mathrm{~m}$ Torr operating pressure and $130 \mathrm{~W}$ of $\mathrm{RF}$ power.

Measurements were also made using the radial RFEA positioned at $\mathrm{z}=9 \mathrm{~cm}$, on the centreline at $\mathrm{r}=0 \mathrm{~cm}$ and facing the HDLT source at $\theta=0^{\circ}$. These measurements were discussed earlier in Section 4.6 and reveal that an ion beam is only present when $3 \mathrm{~A}$ or greater is applied to both of the solenoids. Below $3 \mathrm{~A}$ only one peak is present in the IEDF produced by the RFEA, indicating that no ion beam is formed and that an expanding plasma results. The potential of the ion beam and the local downstream plasma is shown earlier in Figure 4.13 as a function of the solenoid current. It is clear that when an ion beam is present, $\mathrm{V}_{b}$ is constant with increasing solenoid current and therefore the ion beam velocity is constant. The ratio of the ion beam density to the local downstream density was also found to be constant.

Figure 4.22 shows $I(0)$, the total ion flux, and the force of the plasma, $F_{p}$, measured by the MFMI as a function of the solenoid current. As the solenoid current is increased the total ion flux increases as does the force measured by the MFMI. The exception is at low magnetic fields where the force measured by the MFMI increases when the solenoid current is below $1 \mathrm{~A}$ with a very pronounced increase in the force measured at $0.5 \mathrm{~A}$. Several MFMI measurements were made at this magnetic field condition to confirm the validity of the observation. Although 


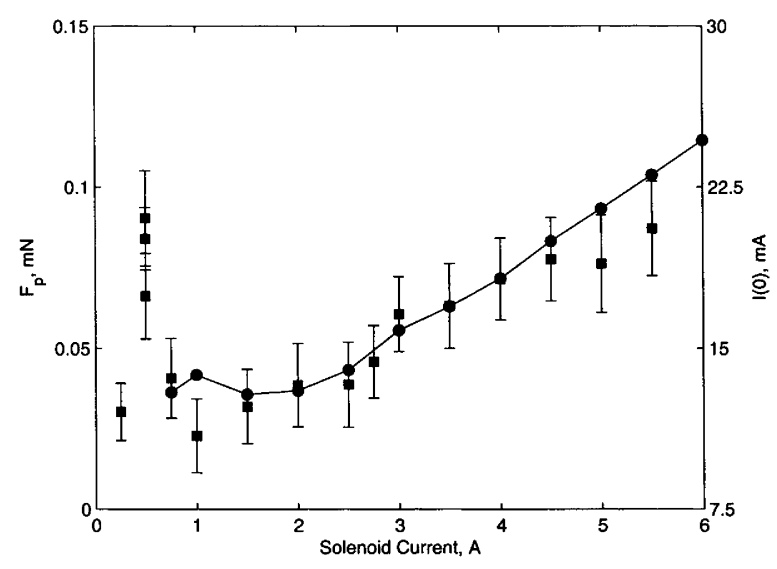

Figure 4.22: The force from the plasma, $F_{p}$, measured with the MFMI as a function of the solenoid current $(\square)$. The total ion flux, $I(0)$, measured in front of the MFMI target is also shown (•). With a current of $3 \mathrm{~A}$ on both solenoids the maximum axial DC magnetic field $B_{z}=138 \mathrm{G}$.

this phenomenon has not been investigated further here, the large increase in the force produced by the plasma may be the result of a jump in the density of the plasma caused by a transition into a helicon wave-sustained mode at these low magnetic field conditions. This density jump at low magnetic field conditions has been observed recently in another helicon experiment by Lafleur et al. (2010).

For the magnetic field conditions where an ion beam is present, that is with a solenoid current greater than $3 \mathrm{~A}, \frac{n_{b}}{n_{d s}}$ and $v_{b}$ are constant, and therefore the increase in the total ion flux results from an increase in the ion beam density. This results in the increase in $F_{t}$ measured when the solenoid current is greater than $3 \mathrm{~A}$. Therefore it can be concluded that the thrust produced by the HDLT can also be increased by increasing the plasma density via increases in the magnitude of the magnetic field used.

\subsubsection{Varying RF Power}

In addition to increasing the magnetic field, the thrust produced by the HDLT can be increased by increasing the inputted RF power. This increases the plasma density inside the HDLT source and the density of the ion beam produced. To investigate this, measurements were made with the MFMI at various RF powers with the following operating conditions: $10 \mathrm{sccm}$ flow rate, $0.4 \mathrm{mTorr}$ operating pressure and a maximum axial magnetic field of $138 \mathrm{G}$. The results are shown in Figure 4.23 . The force from the plasma, $F_{p}$, was found to increase linearly. 


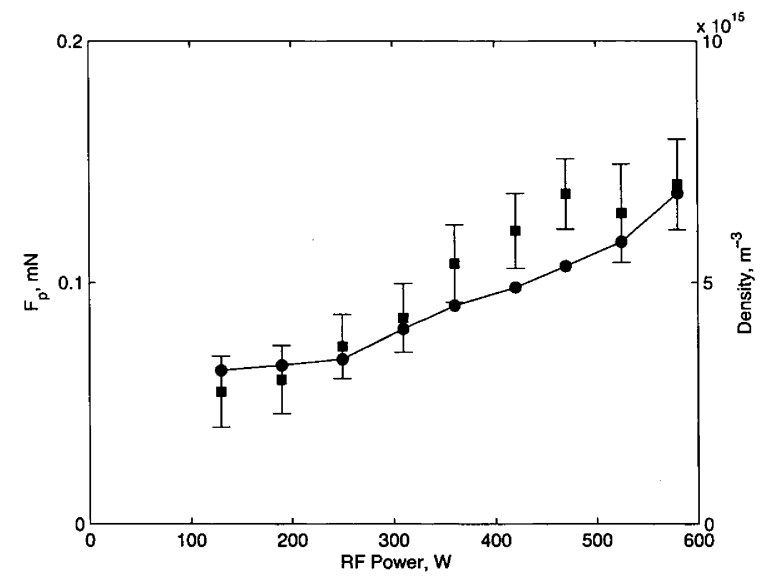

Figure 4.23: The force from the plasma, $F_{p}$, measured with the MFMI as a function of the RF power $(\square)$ with at $0.4 \mathrm{~m}$ Torr and $B_{z}=138 \mathrm{G}$. The plasma density measured in front of the MFMI target with the Langmuir probe is also shown $(\bullet)$

Density measurements as a function of RF power were also made with the Langmuir probe positioned in front of MFMI target at $z=12 \mathrm{~cm}$. The density follows the same trend as the MFMI results suggesting that the force imparted by the exhaust of the HDLT scales with the RF power. It is also important to note that no obvious evidence of a transition to a wave-sustained helicon mode was observed for these conditions and that measurements beyond $400 \mathrm{~W}$ were very difficult to obtain because of issues with the antenna and microarcing inside the vacuum chamber. These phenomena will be discussed further in Chapter 5 .

In summary, the measurements with the MFMI have provided some new insights into the force produced by the exhaust of the HDLT prototype and the forces measured are in good agreement with those determined from the plasma parameters measured downstream with the diagnostic probes. The measurements at various flow rates show that variations in the ion beam velocity and the resulting momentum flux can be measured with the MFMI. The measurements at different magnetic field and RF power conditions demonstrate that the thrust produced by the HDLT prototype can be increased if desired.

However, it is important to note that the MFMI measures the forces produced by the plasma at the location where it is positioned and not at the exhaust of the HDLT prototype. Here, where the MFMI target is positioned downstream at $10 \mathrm{~cm}$ from the exit of the HDLT source tube, the plasma consists of a low density ion beam and a much larger (90\%) downstream component. This is a result of the low pumping speed of the space simulation chamber and downstream ionisation caused by the immersed RF antenna (discussed further in Chapter 5). 
With a higher pumping speed, the operating pressure downstream will be lower and therefore the density of the downstream plasma, which is accelerated by the sheath around the MFMI target and strikes the front and back of the MFMI target, would be lower.

In addition, for the current operating conditions the flux of the ion beam has been shown to decay exponentially along the z-axis of the space simulation chamber as a result of ion-neutral charge exchange collisions (Figure 4.17). Therefore, the flux of ions has already been reduced by a factor of $\frac{1}{e}$ by the time it reaches the location of the MFMI target, which is placed one mean free path downstream. However, the nature of charge exchange is such that an ion acquires an electron from the background gas and this ion then becomes a fast neutral atom and continues along the same trajectory. This fast neutral may then undergo neutral-neutral collisions and scattering later in its trajectory. If the ion was initially heading towards the MFMI target, after charge exchange occurs it may continue along the same trajectory, now as a fast neutral, and could strike the MFMI target, thus contributing to the momentum transferred to the target. The percentage of fast neutrals that are not scattered or experience other collisions is very difficult to quantify. Determining the velocity and density of these fast neutrals and hence the amount of momentum they transfer to the MFMI is therefore not trivial and is beyond the scope of this work. As a result, the contribution from the fast neutrals to the momentum flux measured by the MFMI, which is likely to be quite low, has been neglected here for simplicity. This matter should, however, be investigated in future studies. At higher pumping speeds and correspondingly lower operating pressures downstream, the mean free path for ion-neutral charge exchange collisions will be longer and as a result the flux of ions impacting the front of the MFMI will be larger and less fast neutrals will form. In such a case, the force measured by the MFMI downstream is more likely to be an accurate reflection of the thrust produced by the HDLT prototype at the thruster exhaust.

\subsection{Measurements with Xenon}

As discussed earlier in Section 1.4.2, xenon is the propellant of choice for electric propulsion systems on spacecraft because of its high molecular mass and somewhat lower ionisation energy compared to argon. It is therefore important to verify that the HDLT prototype operates as expected with xenon when immersed inside the space simulation chamber. Charles et al. (2006) have conducted experiments with the HDLT prototype connected to the Chi Kung vacuum chamber previously to confirm that a double layer and an ion beam can be produced in 
xenon. To confirm the presence of an ion beam in xenon, measurements were made at $130 \mathrm{~W}$, with $138 \mathrm{G}$ and at $0.07 \mathrm{mTorr}$, the same operating pressure used by Charles et al. (2006), with an RFEA positioned $10 \mathrm{~cm}$ downstream of the HDLT source at $\mathrm{z}=13 \mathrm{~cm}$. The IEDF revealed a clear ion beam at $55.8 \mathrm{~V}$ and a local plasma potential of $40.6 \mathrm{~V}$, resulting in $V_{D L}=15.2 \mathrm{~V}$, which is in good agreement with the results obtained with Chi Kung using xenon.

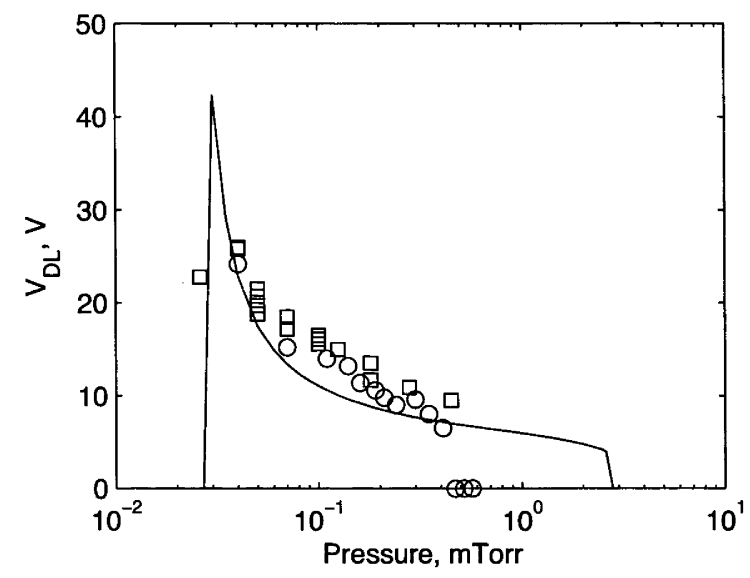

Figure 4.24: Double layer strength, $V_{D L}$, versus pressure for xenon with HDLT immersed in the space simulation vacuum chamber $(\bigcirc)$. The results for the HDLT attached to the Chi Kung vacuum chamber $(\square)$ and theoretical values (-) are from Charles et al. (2006).

The pressure dependence of $V_{D L}$ was then characterised and the results are shown in Figure 4.24 along with the results from the same measurements with the HDLT prototype attached to the Chi Kung vacuum chamber and for the previously mentioned theory but using xenon cross sections (Lieberman and Charles, 2006). When compared to the results for argon (Figure 4.12), it is clear that the pressure envelope over which the double layer occurs is lower for xenon than with argon. The results obtained here and those undertaken previously with xenon are consistent and in good agreement and confirm that the double layer can be reproduced in xenon in this experimental configuration. Chapter 6 details further measurements made with the HDLT prototype using xenon at higher operating RF powers than that used here.

\subsection{Estimate of Exhaust Velocity and Thrust}

From the characterisation of the HDLT prototype undertaken in this chapter it is possible to estimate the ion beam exhaust velocity and thrust with a few important assumptions for the idealised case where the HDLT prototype is operating 
in space. For a monoenergetic ion beam, with zero beam divergence and assuming that the plasma expelled by the HDLT prototype does detach from the source tube and the magnetic field lines, and that the space potential at infinity is zero, the ion beam exhaust velocity can be expressed as

$$
v_{i_{e x}}=\sqrt{\frac{2 e V_{p}}{m_{i}}}
$$

where $V_{p}$ is the potential inside the HDLT source tube and $m_{i}$ is the ion mass.

Figure 4.25 shows the calculated ion beam exhaust velocity for both argon and xenon as a function of the operating pressure based on the data presented in Sections 4.5 and 4.10. The values based on the results from the Chi Kung experiment are also shown for reference (Charles, 2009). It is clear in Figure 4.25 that the exhaust velocities attainable with argon are higher than that attainable with xenon as the exhaust velocity scales, to a first approximation, with the inverse of the square root of the ion mass for constant geometry and magnetic field conditions. However, since the ion beam can be generated at lower operating pressures than with argon, the mass utilisation efficiency with xenon will be higher.

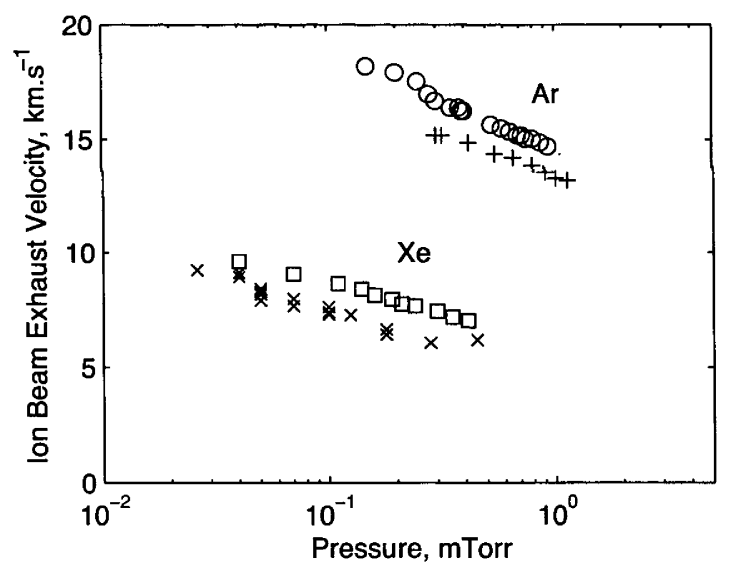

Figure 4.25: The calculated exhaust velocity versus pressure for argon $(\bigcirc)$ and xenon ( $\square$ ) with the HDLT immersed in the space simulation vacuum chamber. The results for the HDLT attached to the Chi Kung vacuum chamber are also shown for argon (+) and xenon $(x)$.

An estimate of the thrust produced by the ion beam exiting the HDLT prototype can be made, based on the measured plasma parameters inside the HDLT source, since

$$
T_{i}=v_{i_{e x}}\left(n_{i} m_{i} A v_{B}\right)
$$

where $n_{i}$ is the density measured just inside the HDLT source and $A=\pi R_{\text {source }}^{2}$ is the cross-sectional area of the HDLT source $\left(15.3 \times 10^{-3} \mathrm{~m}^{2}\right)$. It is assumed here 
that the ions exiting the HDLT source enter the double layer at the Bohm velocity, $v_{B}$, although debate is still continuing as to the validity of this assumption and in particular if the velocity may be up to twice $v_{B}$ (Charles, 2009).

For the case with the HDLT prototype operating with argon at the standard conditions $\left(130 \mathrm{~W}\right.$ at $0.4 \mathrm{mTorr}$ with $\left.B_{z}=138 \mathrm{G}\right), v_{i_{e x}} \sim 16,200 \mathrm{~ms}^{-1}$, the measured density inside the HDLT source is approximately $1.0 \times 10^{17} \mathrm{~m}^{-3}$, $m_{i}=6.68 \times 10^{-26} \mathrm{~kg}$ and $v_{B}=3,530 \mathrm{~ms}^{-1}$ and therefore $T_{i} \sim 5.9 \mathrm{mN}$. Using Equation 1.11, the force from the neutral propellant at this operating pressure ( $0.4 \mathrm{mTorr})$ and room temperature $(300.15 \mathrm{~K}), T_{n}=0.53 \mathrm{mN}$, which is about $10 \%$ of $T_{i}$. When operating under the same conditions with xenon, $n_{i}=0.7 \times 10^{17} \mathrm{~m}^{-3}$ as measured with a Langmuir probe inside the source, $m_{i}=2.18 \times 10^{-25} \mathrm{~kg}$ and $v_{B}=2,010 \mathrm{~ms}^{-1}$ and therefore $T_{i} \sim 4.2 \mathrm{mN}$. $T_{n}=0.60 \mathrm{mN}$ for these conditions with xenon.

Based on the measured plasma parameters, the value estimated for $T_{i}$ for this idealised case, where the HDLT prototype is operating in space, is significantly higher than the forces measured in the HDLT exhaust by the MFMI $\left(F_{p}=57.3 \mu \mathrm{N}\right.$ for argon). As discussed in Section 4.9, the pumping speed of the space simulation chamber is low ( $\left.330 \mathrm{~L} . \mathrm{s}^{-1}\right)$ and therefore the high operating pressure present downstream reduces the flux of ions, via ion-neutral charge exchange collisions, that impacts the MFMI target. This situation would be improved if the operating pressure downstream could be decreased significantly by the use of cryopumps or cryopanels for example, so that at the exit of the HDLT prototype all of the background gas was removed as if it were a black hole. With a lower operating pressure the mean free path for ion-neutral charge exchange collisions would increase to lengths beyond that of the space simulation chamber and all of the ions in the beam could reach the MFMI. In such a circumstance, the MFMI may be able to provide more realistic quantitative measurements of the thrust produced by the HDLT prototype.

However in the current configuration of the experimental apparatus, the qualitative behaviour of the force measured by the MFMI in the exhaust plume is consistent with the behaviour of the plasma in the source (and downstream). That is, when the pressure, inputted RF power or the magnitude of the magnetic field are varied, the force measured by the MFMI varies in the same fashion as the plasma density and ion beam velocity change. This demonstrates how the thrust of the HDLT prototype can be scaled and allows an examination of the influence of the different operating parameters on the thrust produced. 


\subsection{Conclusions}

The results in this chapter confirm for the first time that an ion beam is formed as a result of a current-free double layer when the HDLT prototype is immersed in a space simulation chamber. The ion beam and plasma have been characterised axially and radially using a RFEA and a Langmuir probe. The ion beam has been detected up to $21 \mathrm{~cm}$ downstream and the double layer accelerates argon ions to velocities of $\sim 8.7 \mathrm{kms}^{-1}$ at $0.4 \mathrm{mTorr}$ and as high as $\sim 11.4 \mathrm{kms}^{-1}$ at $0.15 \mathrm{mTorr}$. Although difficult to determine the exact location, the double layer is present in the region between $1 \mathrm{~cm}$ and $5 \mathrm{~cm}$ from the exit of the HDLT source. The potential and density profiles both axially and radially are consistent with experiments in various other configurations. An unusual increase in the ion flux has been measured near where the outermost magnetic field lines emanates from the HDLT source and may be an expression of phenomena observed in other experiments. The ion beam has been shown to have a constant radial potential (and velocity) downstream and to match the diameter of the HDLT source, suggesting a very low beam divergence in agreement with the work of others. The electron temperature has also been shown both axially and radially to be approximately $5.5 \mathrm{eV}$ downstream in the region near the wall of the space simulation chamber.

The double layer potential is shown to vary with the pressure in a fashion that is consistent with previous studies and a proposed model for current-free double layer formation. These results provide strong evidence that a double layer can be created independent of the geometry of the experiment; that is, in a configuration that more closely resembles how the HDLT prototype may be used as a spacecraft electric propulsion system. In addition, since the properties of the double layer and ion beam are shown to be consistent with current-free double layers in 'standard' configurations the results from these other studies may also be applied directly to the optimisation of the HDLT for space applications. The pressure dependence of the double layer potential also demonstrates that the exhaust velocity and hence the specific impulse of HDLT prototype can be scaled allowing for variable specific impulse missions, which are attractive for some deep space mission applications.

The effect of varying the magnitude of the magnetic field has been investigated and demonstrates that the ion beam beam velocity remains constant with increasing magnetic field while the total plasma density increases. The previously demonstrated transition, with varying magnetic field strength, from an expanding plasma to a double layer containing expanding plasma has also been reproduced. By retracting the HDLT source tube $3 \mathrm{~cm}$, it has been shown that the magnitude of the double layer potential drop is not changed by moving the HDLT 
source tube relative to the magnetic field and the HDLT structure and antenna. The density profile and hence the position of the double layer is not affected by moving the HDLT source tube, although it could only be moved $3 \mathrm{~cm}$. The decay in the flux of the ion beam downstream has been characterised and the gyro radius of the ion beam detected downstream has been determined. These measurements have been discussed in relation to issues associated with plasma detachment and the resultant effect upon thrust generation. In particular, the ion gyro radius is shown to approach the radius of the space simulation chamber demonstrating that the ions downstream are no longer under the influence of the magnetic field and are likely to have detached from the plasma exhaust.

Measurements with a new diagnostic technique, that is the MFMI positioned in the plasma exhaust of the HDLT prototype, have investigated the thrust produced by the HDLT exhaust. The forces measured have been shown to be in good agreement with the forces calculated from the plasma parameters determined by the RFEA and Langmuir probe at the MFMI position. Although the force measured in the exhaust is significantly lower than what would be expected to be produced by the HDLT prototype if it was operating in space (based on the plasma parameters inside the HDLT source), several qualitative insights have been gained with this new diagnostic. The force from the plasma increases as the operating pressure is decreased and the velocity of the ion beam increases; consistent with conclusions drawn from earlier RFEA measurements. In addition, when increasing the inputted RF power or the magnitude of the magnetic field, the force measured by the MFMI increases, which demonstrates that the thrust produced by the HDLT prototype is also scalable with these operating parameters.

Finally, measurements operating at low RF power with xenon have confirmed that an ion beam generated by a current-free double layer can be produced when the HDLT prototype is immersed inside a space simulation chamber. Xenon is the propellant of choice for electric propulsion systems. The measured IEDFs are consistent with previous experiments with xenon in other devices and the pressure dependence of the double layer potential is in good agreement with these other experiments and theoretical calculations. These measurements provide a link to Chapter 6, which details experiments undertaken with the HDLT prototype operating at higher RF powers using xenon and the characterisation of the plasma with the RFEA, Langmuir probe and the MFMI. 


\section{Operating RF Antennas Immersed in a Vacuum $^{\dagger}$}

Operating RF antennas in a vacuum presents several challenges which are relevant to the use of plasma propulsion systems like the HDLT and testing such systems inside vacuum chambers. Antennas immersed in vacuum can come into contact with the plasma and if one end of the immersed antenna is grounded direct currents flow from the plasma to ground. This also results in an additional Ohmic loss term that can reduce the plasma density. To the author's knowledge, these issues have not been investigated previously in the context of plasma propulsion. However, in asymmetric plasma discharges, which are used in materials processing, the RF biased substrate or electrodes are frequently immersed in the vacuum (and plasma) and biased negatively via a DC blocking capacitor. This enables the energies of the ions impinging the substrate to be controlled independently of the current to the substrate, which is important for various processing techniques (Lieberman and Litchtenberg, 2005). Immersed RF antennas have also been employed in plasma immersion ion implantation $\left(\mathrm{PI}^{3}\right)$ devices (Collins and Tendys, 1994).

In magnetically confined fusion experiments, the RF antennas used are usually shielded with a Faraday cage to prevent the antenna coming into contact with the plasma. This does, however, reduce the power coupling of the plasma to the antenna and hence the efficiency of the system. The RF antenna in the H1 Heliac experiment at the Australian National University, however, is in direct contact with the plasma and is used to increase the temperature of the plasma. The antenna, which is driven at $7 \mathrm{MHz}$, is shaped like a loop in the plane of the last closed flux surface and one end of the antenna is grounded (Hamberger et al.,

\footnotetext{
${ }^{\dagger}$ Some of this chapter is being prepared for publication as West et al. (2010).
} 
1990). This allows a direct current to flow from the plasma via the antenna to ground. Currents of approximately $90 \mathrm{~A}$ and plasma potentials of $100 \mathrm{~V}$ have been measured during typical operation of the H1 Heliac.

\subsection{Parasitic Plasma Formation}

When attempting to operate at higher RF powers (typically greater than $500 \mathrm{~W}$ ) or at high flow rates and operating pressures (greater than 3 mTorr with argon and only 0.2 mTorr with xenon), the plasma discharge would escape from inside the HDLT source tube and preferentially form around the antenna in the cavity between the HDLT structure and the HDLT source tube. Figure 5.1 shows an image of the plasma in this cavity as viewed from the upstream window of the space simulation chamber. This is obviously not desirable and prevents the HDLT prototype from being operated at high powers and higher flow rates in the present configuration.

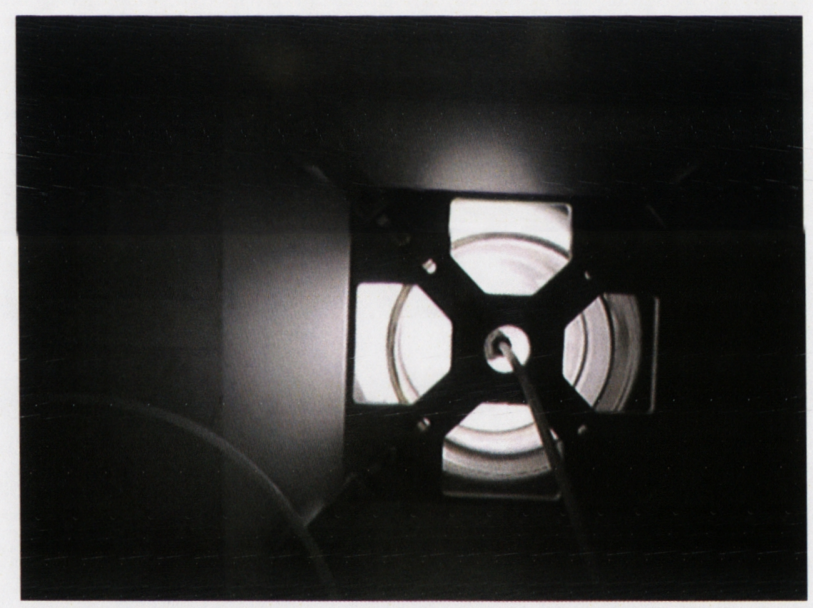

Figure 5.1: The plasma after escaping from inside the HDLT source tube and forming around the antenna in the cavity between the HDLT structure and the source tube.

This phenomenon also produces lots of discharges and sparking on the grounded HDLT structure and damages this structure as well as the antenna. Figure 5.2a shows some of the burn marks on the HDLT structure caused by the parasitic plasma. Discolouration, caused by the sparking, is also visible on the antenna in the top left region of the image. Figure 5.2b shows the discolouration on the antenna more clearly and the areas where the antenna has been burnt, particularly around the antenna strap to the left of the image centre.

In some instances, even when the plasma was contained inside the HDLT source 


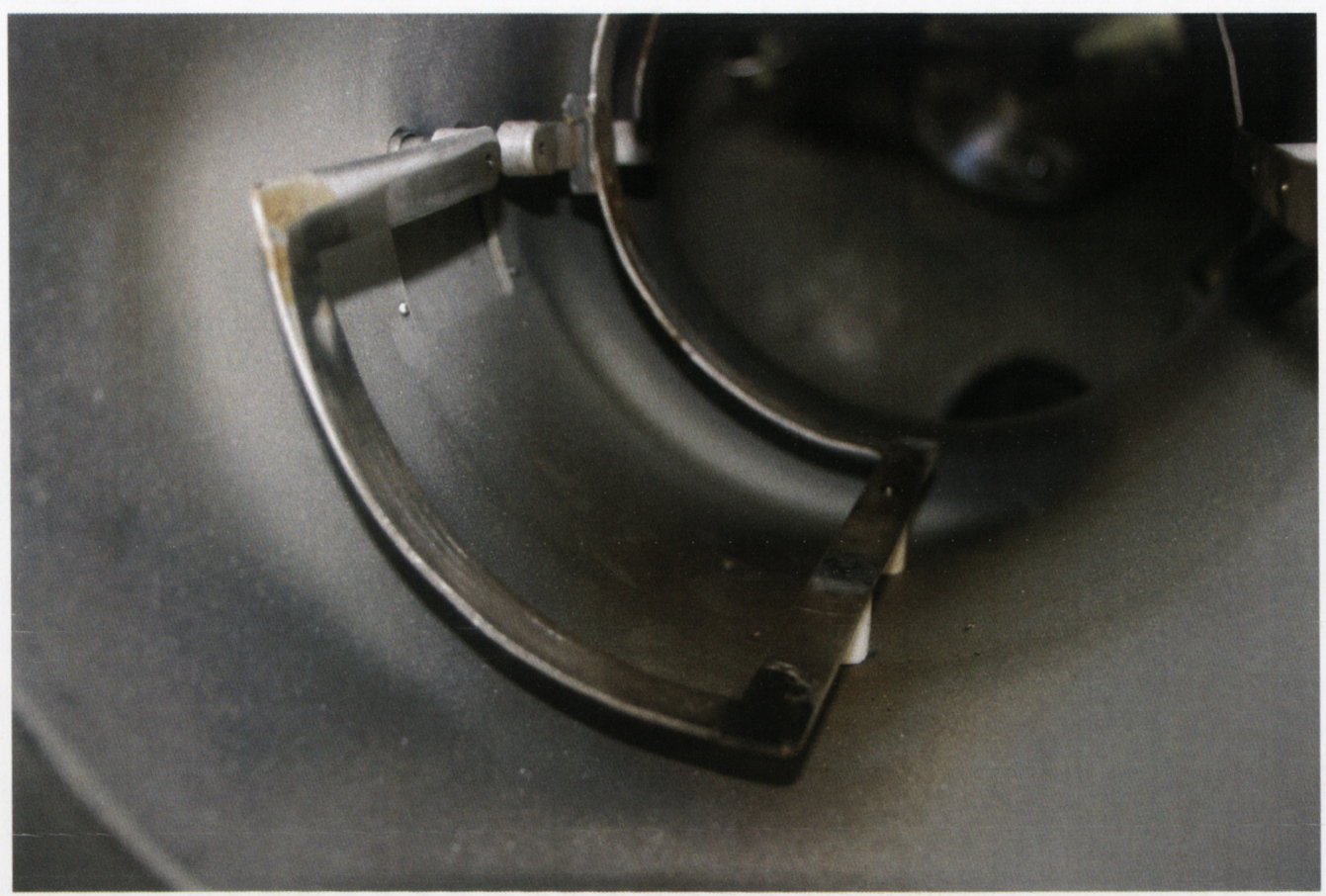

(a)

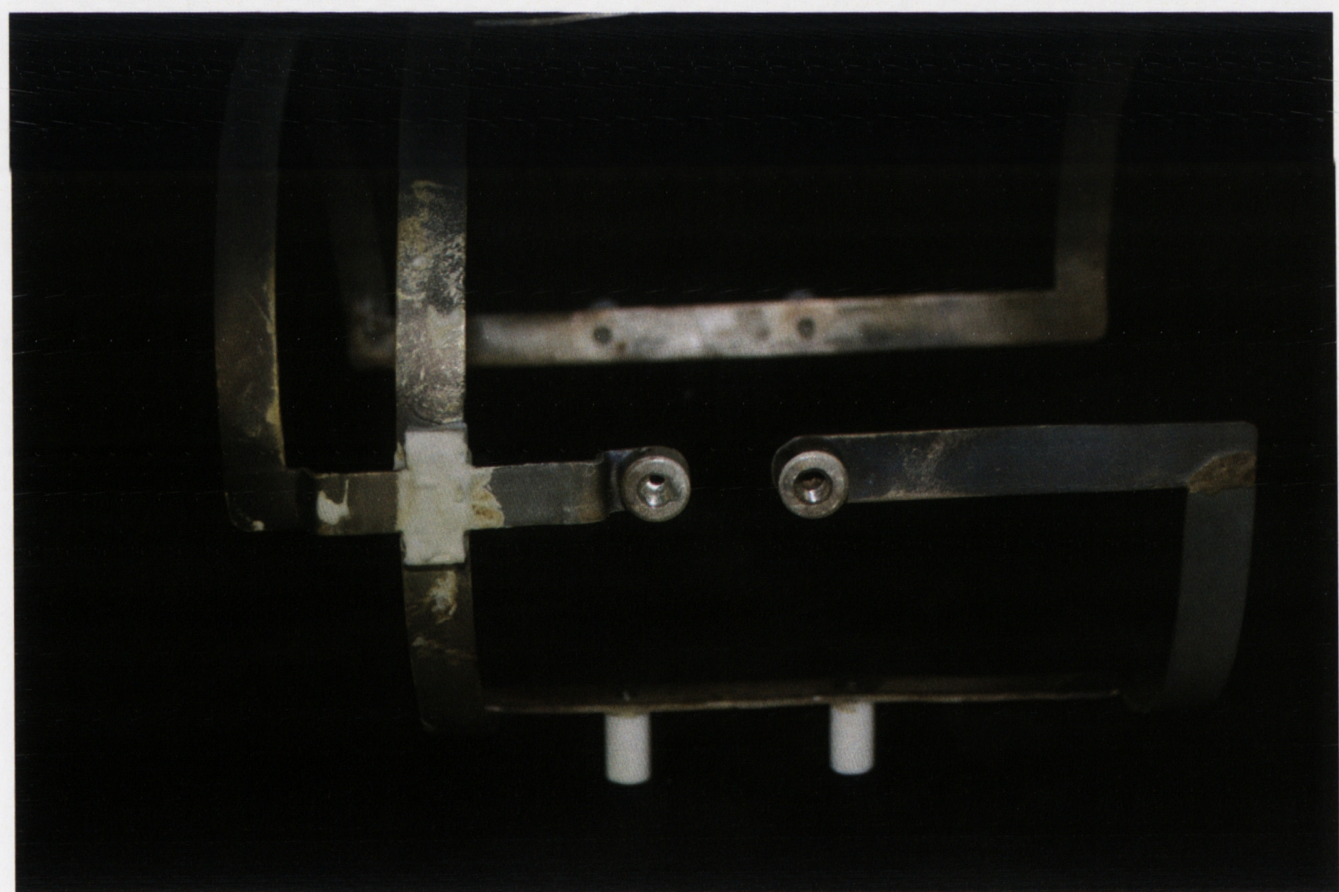

(b)

Figure 5.2: Images of the damage to (a) the HDLT structure and (b) the antenna from plasma forming outside the source tube and around the antenna. 
tube and matched correctly, a small localised plasma was observed around the antenna. This parasitic plasma affects the tuning of the plasma and results in some sputtering of the silver coating that is on the antenna. The amount of RF power applied to the plasma formed in the HDLT source is also reduced as a result of the parasitic plasma and this makes it difficult to assess the actual power input into the HDLT prototype and consequently its power efficiency.

Under all operating conditions a halo of plasma is also observed around the HDLT prototype, including upstream behind the thruster and around the HDLT structure. This occurs because neutral propellant exiting the HDLT prototype remains in the chamber because of the limited pumping speed. For reference, the average residence time for a neutral argon atom in this system is about $15 \mathrm{sec}-$ onds. Since the RF antenna is located inside the space simulation chamber and the RF power is transmitted in all directions by the antenna, this residual gas is ionised independently of the desired ionisation that occurs inside the HDLT source. In reality, any plasma outside the HDLT source could have formed from a combination of neutral gas interactions with the plasma in the HDLT source, the plasma from the source diffusing downstream and plasma created independently of these around the antenna and behind the thruster.

In order to investigate the plasma observed behind the HDLT prototype, measurements were made with the Langmuir probe positioned at $\mathrm{r}=32 \mathrm{~cm}$ and moved axially from behind the HDLT prototype at $\mathrm{z}=-42 \mathrm{~cm}$, past the exit of the HDLT source tube and to a position $30 \mathrm{~cm}$ downstream at $\mathrm{z}=33 \mathrm{~cm}$. Figure 5.3 shows the density measured when operating with argon at $0.4 \mathrm{mTorr}$, with the standard magnetic field configuration $\left(B_{z}=138 \mathrm{G}\right)$ and at $130 \mathrm{~W}$ and $470 \mathrm{~W}$ of RF power. Compared to the densities measured inside the HDLT source on the centreline in Section 4.2.2 (at $130 \mathrm{Wn} n=1.3 \times 10^{17} \mathrm{~m}^{-3}$ ), the plasma density in the region behind the thruster is approximately two orders of magnitude smaller at $\sim 1.5 \times 10^{15} \mathrm{~m}^{-3}$ at $\mathrm{z}=-42 \mathrm{~cm}$. It is also clear in Figure 5.3 that when the RF power is increased from $130 \mathrm{~W}$ to $470 \mathrm{~W}$, the plasma density increases everywhere inside the space simulation chamber, including behind the thruster.

As a result of these parasitic plasmas and in particular the halo of plasma located around and behind the HDLT prototype, it is impossible to assess the power efficiency of the HDLT when testing it in this configuration. The applied RF power is deposited into the total plasma and not exclusively into the plasma in the HDLT source. Therefore the power going into the HDLT source can not be measured exactly since the total plasma also includes the plasma in the space simulation chamber around the HDLT prototype and any plasma formed around the antenna. 


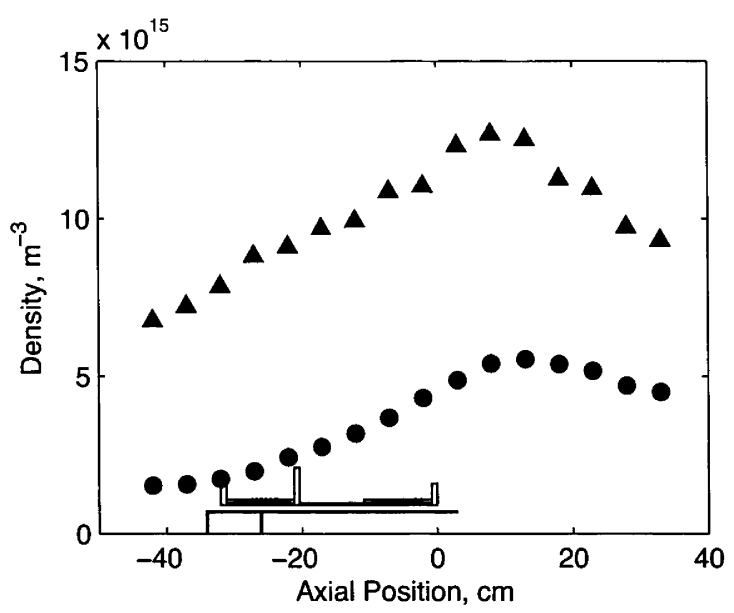

Figure 5.3: The density versus the axial position at $\mathrm{r}=32 \mathrm{~cm}$ at $130 \mathrm{~W}(\bullet)$ and $470 \mathrm{~W}(\mathbf{\Delta})$. The schematic of the HDLT prototype is shown for reference.

\subsection{Microarcing}

As the RF power applied to the HDLT prototype is increased, it was found that many small pinpoint discharges appear on the inside of the walls of the vacuum chamber. This phenomenon is known as microarcing (Yin et al., 2004, 2006) and has been attributed to the breakdown of the sheath at the vacuum chamber wall as a result of an increase in the plasma and floating potentials (Aanesland et al., 2004). As illustrated in Figure 5.4 and outlined by Aanesland et al. (2005), the immersed antenna is powered by an RF voltage of amplitude, $V_{r f}$, has an area of $A_{s}$, is in direct contact with the plasma and is DC grounded. The wall of the vacuum chamber is also grounded and has an area of $A_{w}$. Here $A_{w}>>A_{s}$ and so the impedance of the sheath on the immersed antenna dominates that of the walls of the chamber because of their vastly different areas and hence capacitances.

Figure 5.4 shows the voltage distribution in the system at various times throughout the RF cycle. At the immersed antenna, when the applied RF voltage, $V_{r f}(t)=V_{r f} \cos (\omega t)$, is at a maximum, the instantaneous plasma potential, $V_{p}(t)$, is at its maximum and is lower than the RF amplitude, $V_{r f}$. This results in an electron current flow from the plasma towards the immersed antenna. Conversely, $V_{p}(t)$ is much larger than $V_{r f}$ when $V_{r f}(t)$ is most negative and an ion current flows from the plasma to the immersed antenna. With each RF cycle, the magnitude of the electron current drawn over the positive half cycle is much larger than that of the ion current drawn over the negative half cycle and the net negative charge escapes from the plasma via the grounded antenna. Since charge equilibrium can not be maintained, the plasma potential will rise progressively 


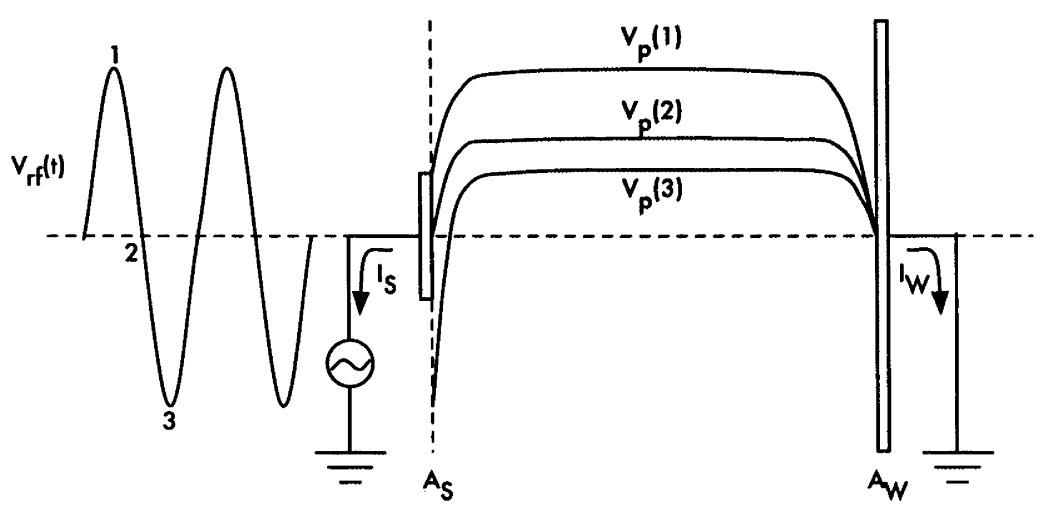

Figure 5.4: Schematic of the voltage distributions in front of the immersed antenna and the vacuum chamber wall at various times during the RF cycle.

as electrons are lost without a corresponding loss of ions. Eventually the plasma potential will rise to such a point that the plasma sheath at the chamber wall can not be maintained and the sheath rapidly collapses resulting in an arc breakdown at the chamber wall (Yin et al., 2005).

The microarcs result in a large release of energy over a small area at the chamber wall over the space of a millisecond or less. These discharges can damage sensitive electrical components and interfere with diagnostic measurements as the bulk plasma parameters are momentarily varied. At the location of the microarc, metallic vapour and other impurities are also released that can result in undesirable coatings being deposited on the space simulation chamber, on the propulsion system being tested and on any plasma diagnostics. Examination of the walls of the space simulation chamber revealed many examples of pitting as a result of these microarcs.

In order to investigate the microarcing phenomena, time resolved measurements were made of the floating potential with the Langmuir probe during the microarcing events. The Langmuir probe was installed downstream of the HDLT prototype on the space simulation chamber centreline at $\mathrm{r}=0 \mathrm{~cm}$ and $\mathrm{z}=6 \mathrm{~cm}$ and attached directly to the Tektronix TDS1012B Digital Storage oscilloscope. Figure 5.5 shows an example of the results obtained.

It is clear in Figure 5.5 that as a microarc event begins the floating potential drops rapidly by approximately $-35 \mathrm{~V}$ and continues to decrease as the microarcing event progresses. Once the arcing event stops, the charging process rebuilds the floating potential and the floating potential increases exponentially with a typical time constant of $\sim 50 \mu \mathrm{s}$, which is much longer than the RF cycle and is probably related to the ion diffusion. This continues until the floating potential increases enough to trigger another microarc event. The microarcing was observed to be 


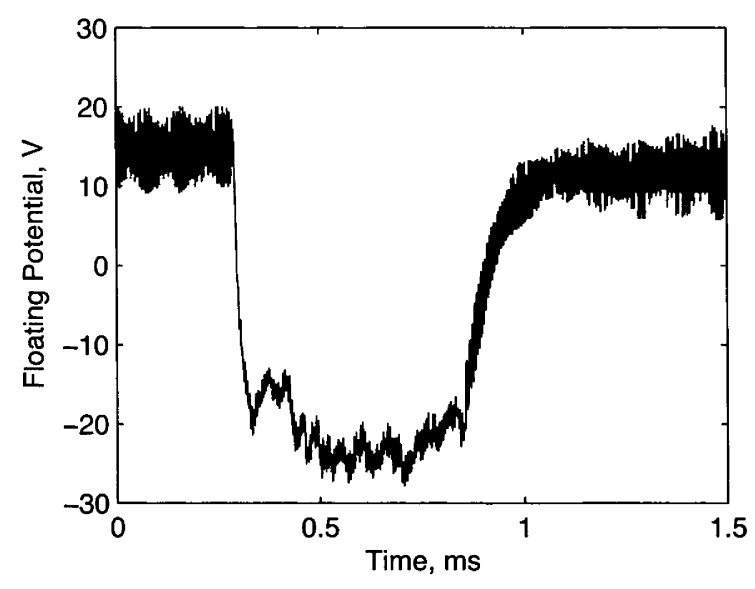

Figure 5.5: The floating potential vs time during a microarc event.

quasi-periodic with large discharges followed by smaller ones. The arcing frequency was found to increase with increased RF power and also increased with a decrease in the operating pressure. These observations are consistent with the observations of other researchers (Yin et al., 2004, 2006).

\subsection{Possible Solutions}

During the testing campaign at ESTEC with the HDLT prototype, neither parasitic plasmas nor microarcing events were observed. This could be because of the significantly higher pumping speeds of the test facility $\left(7,000 \mathrm{~L} \cdot \mathrm{s}^{-1}\right.$ in the hatch and $12,000 \mathrm{~L}^{-1}{ }^{-1}$ in the CORONA chamber with xenon). However, only limited tests were undertaken and so these phenomena could have been present but not observed. Nonetheless, the most obvious solution to the challenges outlined above is to increase the pumping speed of the space simulation chamber significantly. This is best done via the installation of cryogenic panels downstream. After the neutral propellant has been ejected from the HDLT prototype it strikes the panels and freezes onto the surface effectively removing the neutral propellant from the system (provided that the panel is maintained at a low enough temperature). By reducing the residence time of the neutral atoms in the system and reducing the operating pressure downstream, there will be less propellant available (since a sealed gas injector can be used at the back of the plasma cavity) and parasitic plasmas are less likely to form around the antenna and behind the HDLT prototype. Unfortunately, cryogenic panels are very expensive to construct and operate and this option was not available here at this time, but is being considered for the future. 
Several other methods for mitigating the problems described above were also considered. Sealing off the cavity between the HDLT structure and the HDLT source tube could prevent the formation of parasitic plasmas around the antenna. However, to ensure that no residual gas was present, the cavity would need to be differentially pumped and maintained at a pressure lower than the rest of the space simulation chamber. This is not a trivial task and is likely to be as expensive as installing cryogenic panels, as an additional turbomolecular pump would be required. In addition, sealing off this cavity would restrict access to the antenna if repairs were required and would make replacement of the HDLT source tube extremely difficult.

The use of electrostatic shields or Faraday cages, such as those employed elsewhere (Sugai et al., 1994), were considered. However, these approaches have been shown to reduce the capacitive coupling between the antenna and the plasma and are also know to reduce the coupling efficiency to the plasma (Suzuki et al., 1998), which is undesirable. Potting the antenna, that is, covering it with ceramic paste, to prevent plasma forming around the antenna and the removal of the silver coating was also investigated. The ceramic insulator would act as a sacrificial capacitor that would charge up and sputter instead of the silver coating of the antenna. To be effective, the ceramic coating would need to be thicker than the sheath thickness and so about $1 \mathrm{~cm}$ thick. This is a very drastic approach and would make non-destructive repair or modification of the antenna and source tube very difficult.

Another option is to modify the matching box circuit and remove or limit the current path to ground. This can be done in one of two ways. Jaworski et al. (2006) has investigated direct current magnetic insulation of an immersed RF antenna, which reduces the potential difference between the plasma and the antenna via the application of a DC current to the antenna. This DC current produces a magnetic field next to the antenna. It is proposed that this magnetic field traps electrons that would normally escape the plasma to the antenna. The alternative approach, is to use a floating antenna configuration which has been investigated by several groups and has been shown to limit microarcing and other detrimental phenomena (Aanesland et al., 2003, 2004, 2005; Yin et al., 2004, 2005, 2006). By installing a blocking capacitor, the immersed antenna is allowed to float and to develop an appropriate negative self-bias, which enhances the negative voltage excursion and reduces the positive voltage excursion with respect to the plasma potential. This brings the electron and ion currents into equilibrium and limits sheath breakdown and microarcing. The installation of a blocking capacitor to the matching box is reasonably straight forward and so this approach was adopted. 


\subsection{Matching Box Modifications and Consequences}

To minimise the challenges associated with microarcing and parasitic plasma formation and enable the HDLT prototype to be operated at higher RF powers and flow rate, a $9 \mathrm{nF}$ blocking capacitor, $C_{\text {block}}$, was added to the matching network. This capacitor acts as a DC block, isolating the antenna from ground, and allows the antenna to develop a negative self-bias with current flowing only during the charge up stage of the capacitor (some tens of $\mu \mathrm{s}$ ). As a result, the positive voltage excursions on the antenna during each $\mathrm{RF}$ cycle are reduced with respect to the plasma potential. This maintains the equilibrium of the electron and ion currents, preventing the plasma potential from rising, the sheath breaking down and hence the instances of microarcing. It should be noted that with this additional capacitor, the matching circuit is still resonant. The measured $\mathrm{Q}$ factor, which is discussed further in Section 6.6, with and without the blocking capacitor is unchanged $(\mathrm{Q} \sim 8)$ for a $130 \mathrm{~W}$ argon plasma.

Figure 5.6 shows a schematic of the modified matching network circuit and Figure 5.7 shows the array of smaller capacitors that form the $9 \mathrm{nF}$ blocking capacitor, which is installed in parallel inside the matching box. With the blocking capacitor installed, it was possible to safely increase the RF power beyond $500 \mathrm{~W}$ without the plasma escaping from the HDLT source and forming around the antenna. The instances of microarcing also decreased significantly. Unfortunately even with the blocking capacitor installed, the halo of plasma is still visually present downstream and behind the thruster. Although not investigated in this thesis, an interesting future study could investigate if the installation of the blocking capacitor changes the proportion of plasma found behind the HDLT prototype.

However, the installation of a blocking capacitor does have some consequences. Large negative self-biases can form on the antenna, which enhance the negative voltage excursions on the antenna during each RF cycle. This causes ions in the plasma to be accelerated by the sheath and flow towards the antenna. These ions bombard the antenna and sputter the silver surfaces (Aanesland et al., 2003). Since the sputtering rate is a function of the energy of the bombarding ions, the greater the magnitude of the antenna self-bias, the greater the rate at which the silver is removed from the antenna.

Using the high voltage probe, the self-bias on the antenna, $V_{b i a s}$, was measured as a function of RF power when operating with xenon and the results are shown in Figure 5.8. The magnitude of the self-bias on the immersed antenna increases with the RF power, which is consistent with observations in other helicon sys- 


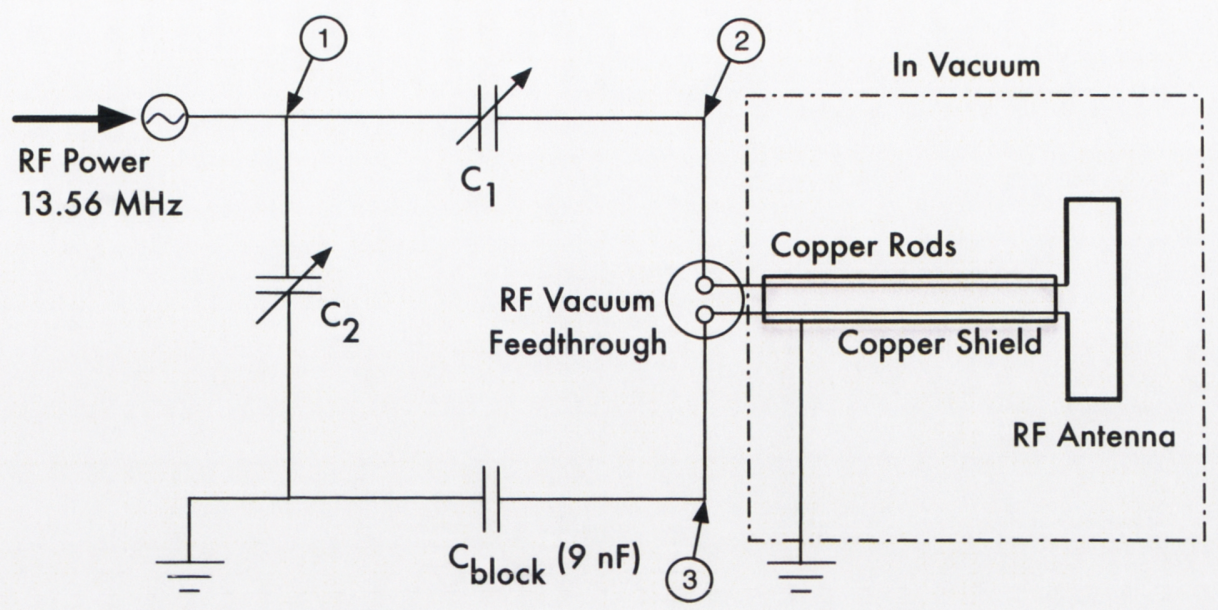

Figure 5.6: Schematic of the matching box circuit showing the tunable capacitors $C_{1}$ and $\mathrm{C}_{2}$ and the DC blocking capacitor $\mathrm{C}_{\text {block}}$. The positions where measurements with the high voltage probe, as described here and in Section 6.6, are also shown.

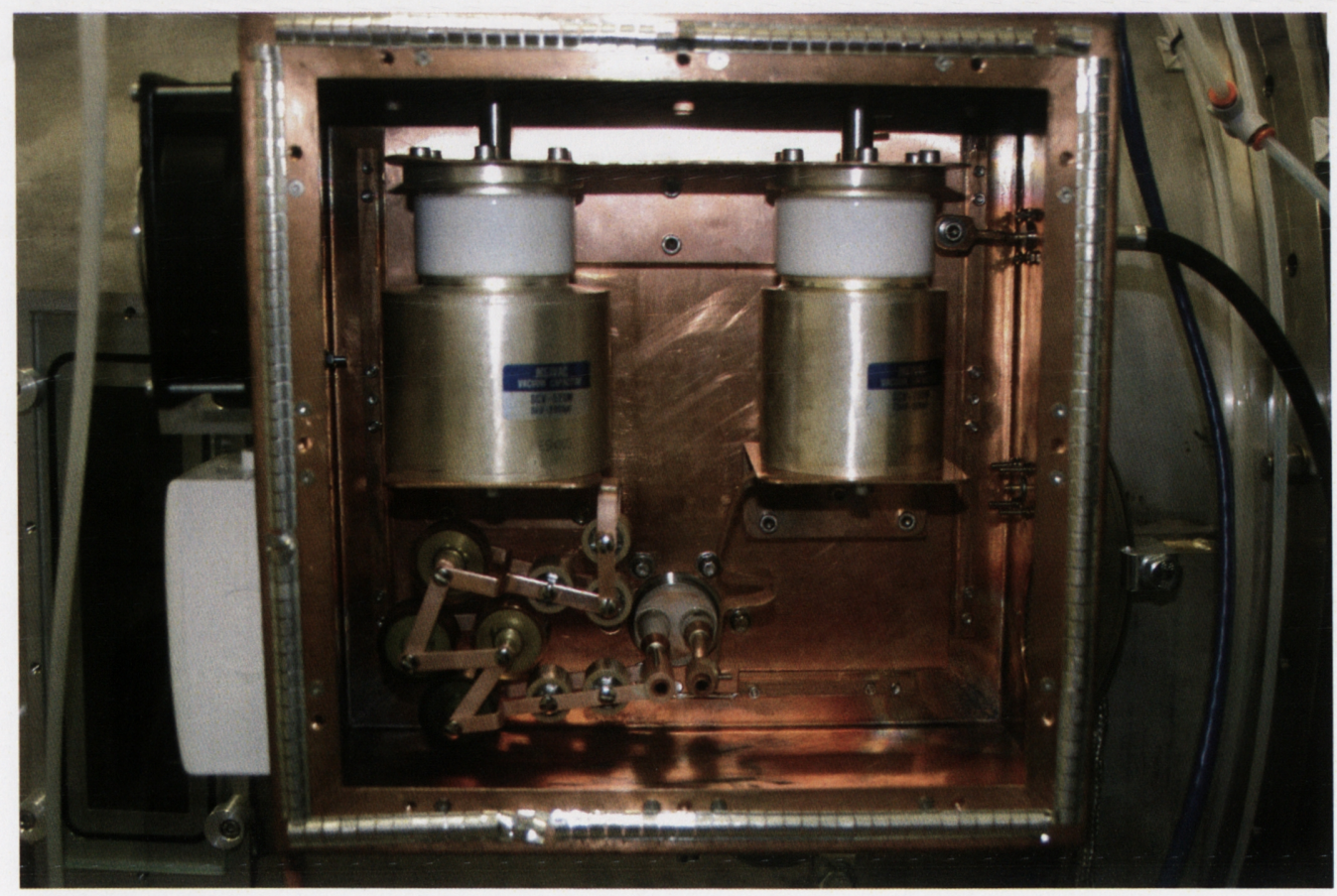

Figure 5.7: The blocking capacitor installed inside the matching box. 


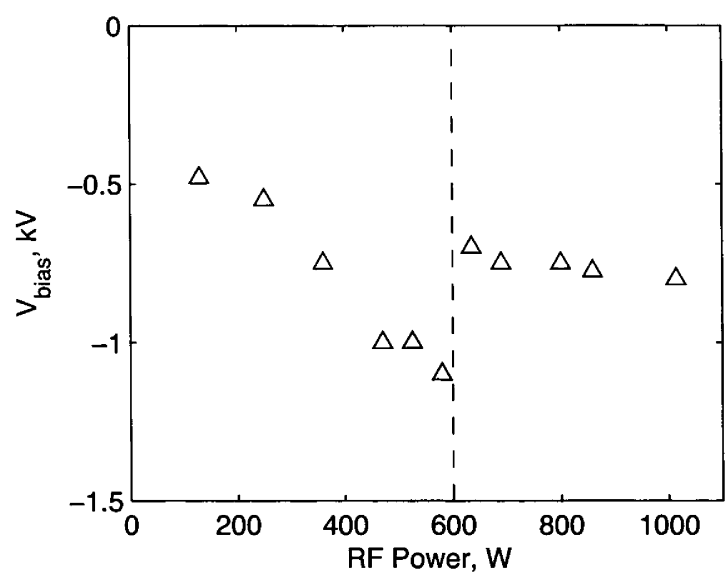

Figure 5.8: The antenna bias voltage measured at position 3 on the matching box versus $\mathrm{RF}$ power when operating at $0.45 \mathrm{~m}$ Torr with xenon.

tems (Aanesland et al., 2003, 2004). The dashed line in Figure 5.8 indicates the RF power at which the plasma transitions to the high density xenon mode which is discussed in Chapter 6 . In this high density mode, the magnitude of $V_{\text {bias }}$ decreases as the matching of the plasma changes dramatically and will be discussed in further detail later. However, it is worth noting that the extent of sputtering was observed to reduce once the plasma transitions to the high density mode and this is consistent with the measured decrease in the antenna self bias.

If not managed correctly, the sputtered silver can accumulate on the HDLT source tube, the HDLT structure and the antenna electrical connectors. These layers of silver can create unwanted paths to ground. The sputtered silver can also cause damage to the antenna and other components as well as change the plasma parameters dramatically. Figure 5.9 shows the burn mark caused by a short circuit that was created when sputtered silver deposited on the insulator that separates the straps of the antenna where the straps overlap.

The sputtered silver can also coat the ceramic spacers that are used to affix the antenna to the grounded HDLT structure, creating unwanted paths to ground. When this occurs, the power from the antenna shorts to the HDLT structure and the plasma can not be ignited and tuned correctly. In order to minimise this problem, ceramic discs with a diameter larger than the ceramic spacers were added to act like an 'umbrella' and protect the ceramic spacers from being coated with the sputtered silver. This proved to be an effective strategy for minimising the unwanted electrical pathways to the grounded HDLT structure. Figure 5.10 shows the repaired antenna after the sputtered silver has been removed via shot blasting. The modified ceramic spacers and ceramic discs are also visible. 


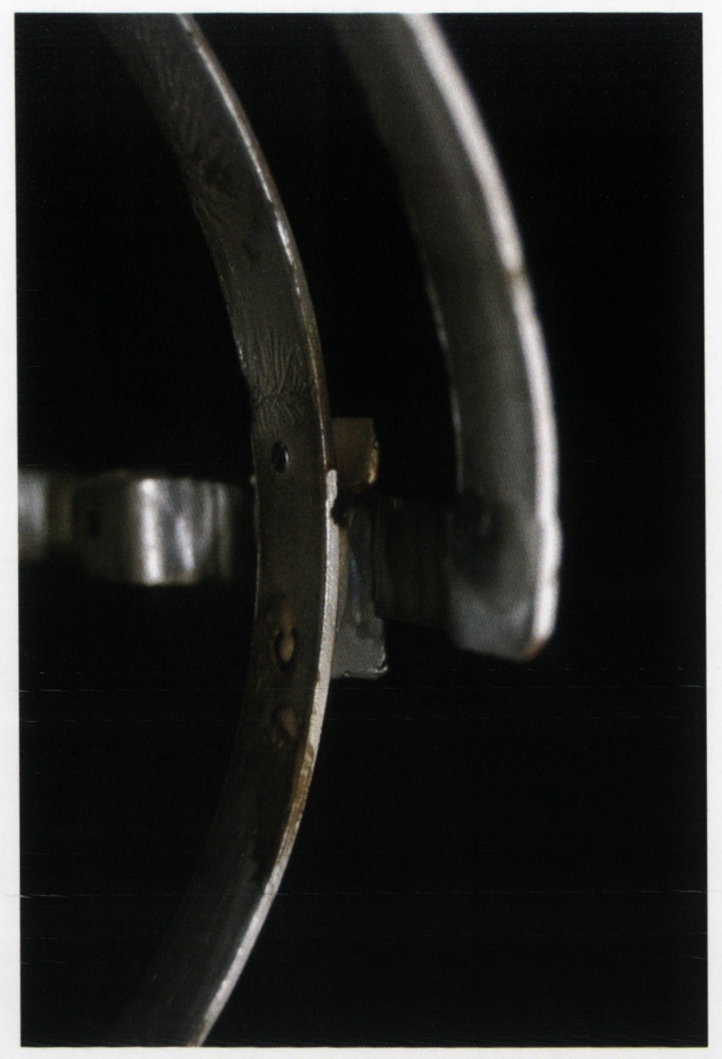

Figure 5.9: A short circuit on the antenna strap caused by sputtered silver.

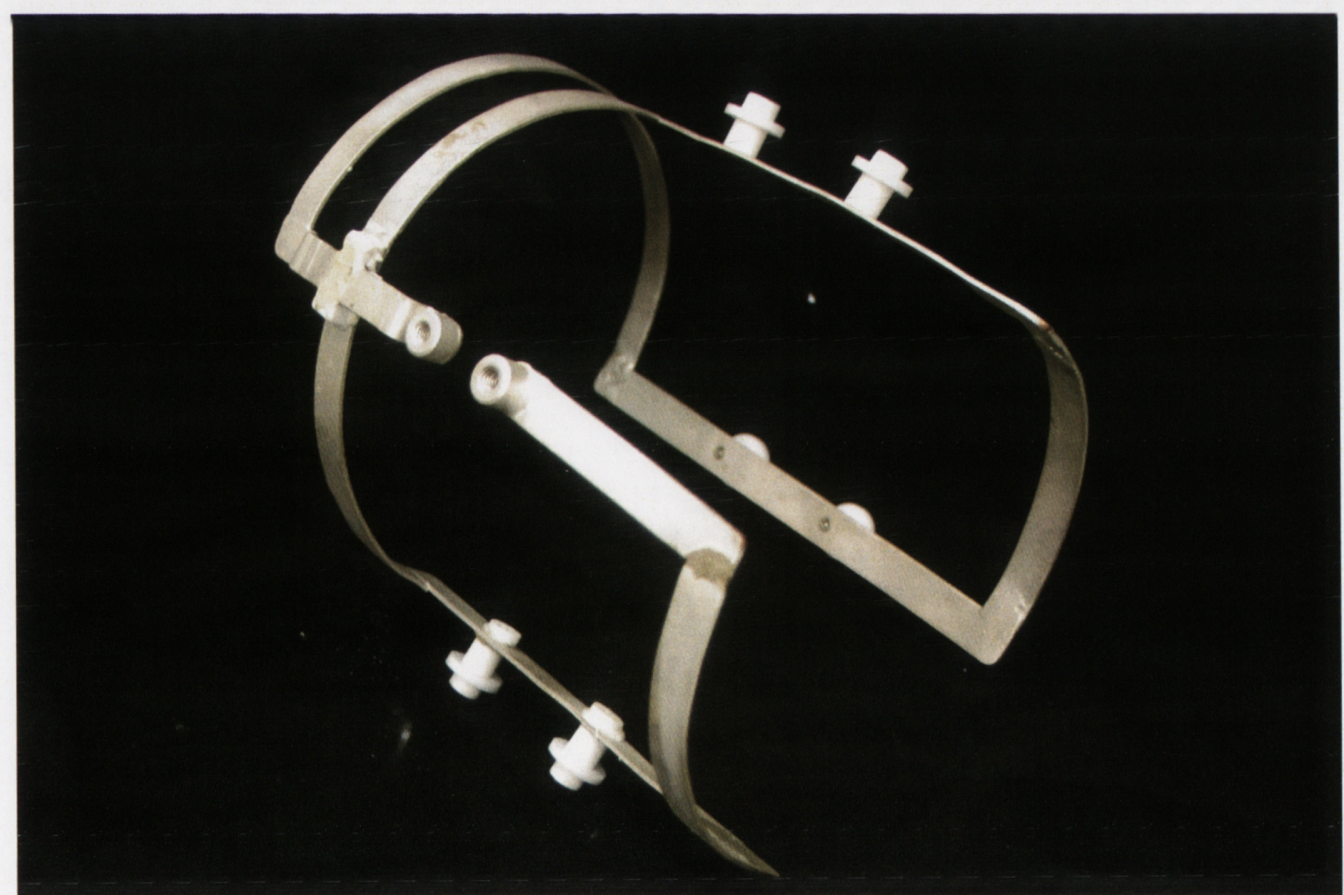

Figure 5.10: The repaired antenna with the modified ceramic spacers installed to prevent the sputtered silver from tracking to ground. 


\subsection{Thermal Loading}

In a vacuum environment, conduction and convection do not occur to any appreciable extent and radiation is the dominant mechanism for heat transfer. As a result the HDLT prototype was originally designed with large radiating panels which make up part of the HDLT structure, which are shown in Figure 2.1. The RF antenna and the Pyrex source tube are the two components that are subjected to the largest thermal loads when the HDLT is operating. It was found that care must be taken when operating the HDLT prototype for extended periods because the antenna and source tube have limited radiation surfaces and limited conduction paths to transfer the heat to the radiating panels. Pulsing the plasma could minimise thermal loading on the HDLT prototype but was not investigated in this thesis.

The HDLT prototype was originally designed so that the Pyrex source tube was separated from the antenna by a few millimetres and not in contact with it. It was found that if the source tube did come into contact with the antenna hot spots would develop at these locations. In one instance, one of the stainless steel screws, used to attach the antenna to a ceramic spacer that was attached to the HDLT structure, was in contact with the source tube and caused that region to heat up, expand and eventually crack. The broken HDLT source tube, which also has a coating of silver visible from the sputtering discussed in Section 5.4, is shown in Figure 5.11. To solve this problem, ceramic spacers where attached to the antenna over the location of the stainless steel screws to ensure that the source tube was would not come into direct contact with the antenna. These spacers are visible on the repaired antenna in Figure 5.10.

\subsection{Conclusions}

In this chapter, several of the challenges associated with operating the RF antenna of the HDLT prototype immersed in a vacuum have been identified. The formation of parasitic plasmas around the antenna and behind the HDLT prototype have been observed. The limited pumping speed of the space simulation chamber means that residual propellant is ionised by the field of the antenna and can create plasmas independent of the desired plasma inside the HDLT source. The density of the plasma behind the HDLT prototype near the wall of the space simulation chamber has been measured and found to be approximately $1 \%$ of that found in the HDLT source. As as consequence, the power efficiency of the 


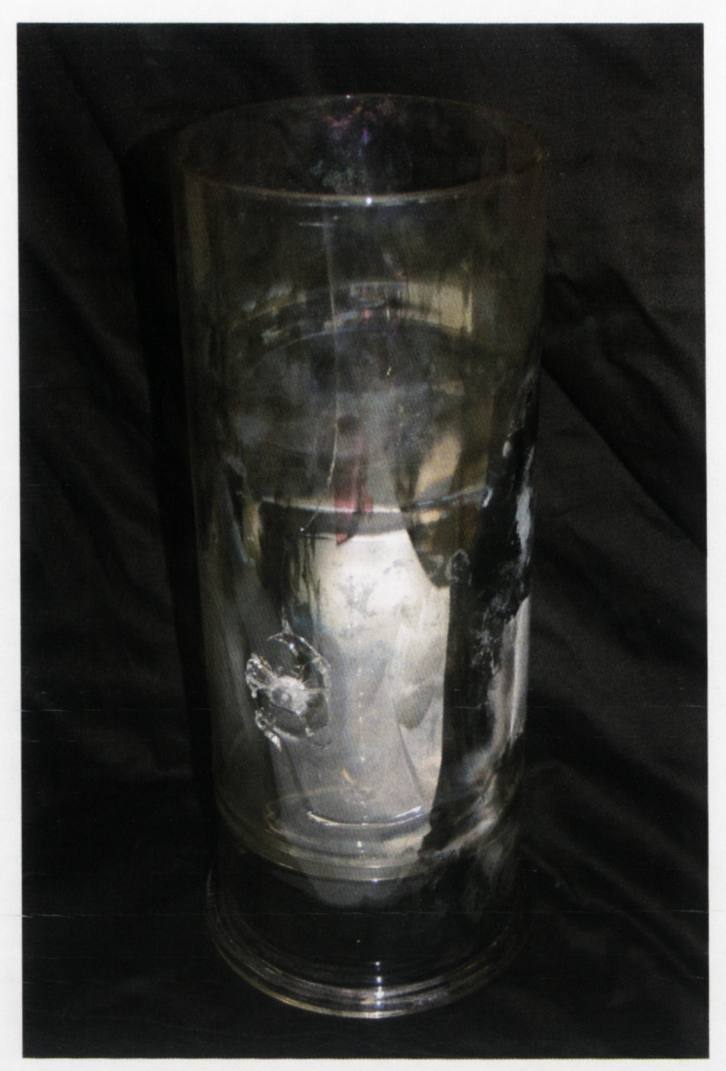

Figure 5.11: The broken HDLT source tube after a hot spot developed where the supporting screw on the antenna made contact with the Pyrex tube. A coating of silver is also present on the source tube from extensive sputtering.

HDLT prototype can not be accurately assessed when these parasitic plasmas are present since the inputted RF power goes into not only the plasma in the HDLT source tube but all the plasmas present.

Small discharges observed inside the space simulation chamber when the RF power was increased have been identified as microarcs caused by the collapse of the sheath at the wall of the space simulation chamber. The physics governing these microarcing events has been described and a time resolved measurement of the floating potential during a microarcing event has been obtained. The microarcs were found to be quasi-periodic and when the RF power was increased or the operating pressure decreased, the arcing frequency was found to increase. Several detrimental effects caused by the microarcing were observed including the deposition of coatings on components inside the vacuum chamber and the pitting of the walls of the chamber.

To alleviate these problems several possible solutions were considered. Modification of the matching box circuit in order to float the RF antenna was deemed 
the most straight forward approach and therefore a blocking capacitor was installed. This enabled consistent and continuous operation at higher RF powers and higher flow rates with both argon and xenon gas. However, the installation of a blocking capacitor does result in large negative self-biases forming on the antenna. Measurements were made of the antenna bias as a function of RF power and the bias was found to induce sputtering of the silver coating of the antenna. The sputtered silver produced unwanted electrical paths that damaged the antenna and other components. Modifications have been made to the antenna to mitigate this problem and the issues associated with thermal loading and contact between the antenna and the HDLT source tube. These experiences highlight some of the challenges that will need to be considered for future designs of the HDLT which are also applicable to the design of other electric propulsion systems that use RF antennas.

Even with the improvement brought by the incorporation of the blocking capacitor in the matching box, parasitic plasmas are still present. In particular, the halo of plasma around and behind the thruster was still observed and so determining the power efficiency of the thruster is not possible, as discussed earlier. It is clear that the limited pumping speed of the space simulation chamber places a limitation on the investigations that can be carried out. The installation of cryogenic panels is required in order to significantly increase the pumping speed. By removing the neutral gas faster, the background pressure will be lower and less parasitic plasmas will form. This will have several advantages including allowing the power efficiency of the HDLT prototype to be evaluated. Despite the limited pumping speed and the issues associated with downstream parasitic plasma, the modified matching box still enables the HDLT prototype to be operated at higher RF powers. Chapter 6 details experiments undertaken with the HDLT prototype operating at higher RF powers and the characterisation of a high density mode in xenon. 


\section{High Power Characterisation of the HDLT Prototype ${ }^{\dagger}$}

\subsection{Operating Modes in RF Discharges}

Radio frequency $(\mathrm{RF})$ plasma sources can be divided into three distinct types: capacitive $(\mathrm{E})$, inductive $(\mathrm{H})$ and wave-sustained $(\mathrm{W})$ (e.g. helicon) discharges (Lieberman and Boswell, 1998). In the capacitive mode, which typically occurs at low powers, power is coupled to the mobile plasma electrons. These electrons oscillate back and forth in response to the instantaneous electric fields produced by the RF driving voltage on the antenna. In capacitively coupled systems, the plasma density inside the source is known to increase proportionally with the square root of the input power (Lieberman and Boswell, 1998), such that

$$
n \propto \sqrt{P_{R F}}
$$

Inside the source, the radial density profile is often close to a cosine with the density at the sheath edge approximately $30 \%$ of the density at the centre (Charles et al., 2003).

In the inductive mode, the induced solenoidal electric field created by the current flowing through the antenna accelerates the electrons. In inductively coupled systems, the plasma density increases linearly with increasing input power (Rayner and Cheetham, 1999), such that

$$
n \propto P_{R F}
$$

${ }^{\dagger}$ Some of the results in this chapter have been published, in a slightly altered form, in West et al. (2009a). 
The radial density profile inside the source is characterised by a hollow density profile with two distinct maxima or shoulders (Lieberman and Boswell, 1998).

In wave-sustained discharges such as helicon sources, magnetic coupling between the antenna and the plasma excites a helicon wave which transfers energy to the plasma, most probably through wave particle interactions. When the wavelength of the helicon wave is sufficiently short to fit inside the source, the overall density increases significantly and the central density in the source also increases. This results in a radial density profile with a pronounced central maximum (Degeling et al., 1996). In the helicon mode, as the input power is increased the density often, but not always, increases exponentially, such that

$$
n \propto \exp P_{R F}
$$

Helicon waves are associated with very efficient plasma production and high ionisation rates, therefore higher densities can be achieved than with capacitive or inductive plasma sources. This is of particular interest for propulsion applications as the thrust produced is a function of the density of ions expelled. High ionisation rates also greatly enhance the power and mass utilisation efficiencies of the propulsion system.

Mode transitions in argon helicon discharges as the applied RF power applied is increased have been widely reported (Boswell and Porteous, 1987; Degeling et al., 1996; Cheetham and Rayner, 1998; Rayner and Cheetham, 1999; Franck et al., 2003). As the RF power is increased discrete 'jumps' in the plasma density are observed as the plasma transitions from the $\mathrm{E}$ to the $\mathrm{H}$ mode and from the $\mathrm{H}$ to the $\mathrm{W}$ mode. These transitions are accompanied by changes in the matching of the plasma and in the case of the $\mathrm{H}-\mathrm{W}$ transition a luminous bright zone, or 'blue core', in the centre of the discharge has been reported when operating in argon (Boswell, 1970, 1984). Transitions directly between the capacitive and wave-sustained modes (E-W) without passing through the inductive mode have also been reported (Franck et al., 2003). These mode transitions are known to have considerable hysteresis as the RF power is increased and then decreased.

\subsection{High Density Mode in Xenon}

During the testing campaign at ESTEC discussed in Section 1.6.2, a new high density 'blue' mode was discovered when operating in xenon (Charles et al., 2008). Although limited diagnostics were available, the density inside the plasma source in this 'blue' mode was estimated to be over $10^{18} \mathrm{~m}^{-3}$ when operating at 
an input power of $500 \mathrm{~W}$. This high density mode was present when operating in the 0.015 mTorr to 0.15 mTorr range. Beyond these initial observations, no conclusions could be reached from these tests regarding the operating mode, the presence of helicon waves or the nature of the mode transition. Therefore, the terminology 'high density mode' is used, as the mode observed in xenon at ESTEC may not necessarily be a wave-sustained mode.

In order to investigate the mode transition observed at ESTEC and to characterise the high density mode, experiments have been conducted when operating the HDLT prototype in the smaller vacuum chamber described in this thesis using xenon. The high density 'blue' mode observed at ESTEC has been reproduced when operating with a maximum magnetic field of $138 \mathrm{G}$. It was found that when increasing the RF power, the plasma jumps into this new mode. The change in mode is easily identified as the appearance of the plasma changes from a light purple colour prior to the transition and to a bright white/blue colour once the transition occurs. The transition is also accompanied by a significant change in the matching of the plasma and the value of the parallel capacitor, $C_{2}$, must be adjusted significantly. Prior to the transition at an RF power of $580 \mathrm{~W}$ and with the plasma matched, $C_{2}$ was measured and equalled $113 \mathrm{pF}$. Following the transition and at an RF power of $635 \mathrm{~W}, C_{2}=438 \mathrm{pF}$. The pre-transition condition is termed the low density mode and the post transition condition the high density mode.

The RF power at which the transition to the 'blue' mode occurs was found to vary as a function of operating pressure as shown in Figure 6.1. The results for experiments conducted at ANU $(\bullet)$ and during the ESTEC testing campaign ( $(\mathbf{\square})$ are shown. Note that at ESTEC the operating pressure is an order of magnitude lower as a result of the larger device size and the greater pumping speed. As the pressure was decreased, the RF power required to initiate the mode was found to increase for both cases. This confirms that the 'blue' mode observed here is in fact the same 'blue' mode as that observed at ESTEC. For the two lower pressure cases examined here ( 0.27 mTorr and $0.32 \mathrm{mTorr})$, the RF power could not be increased enough to initiate the mode as the RF generator has a maximum output of approximately $1 \mathrm{~kW}$. As indicated by the arrows in Figure 6.1, if the transition can be initiated at these lower pressures it will require an RF power higher than $1 \mathrm{~kW}$ with this experimental setup.

Attempts were made to initiate the high density mode with argon, however it could not be reproduced at 0.45 mTorr with a magnetic field of $138 \mathrm{G}$ at RF powers up to $1 \mathrm{~kW}$. Even when the pressure was increased to 2 mTorr, no mode transition could be found. The mode could be reproduced at high RF powers with 


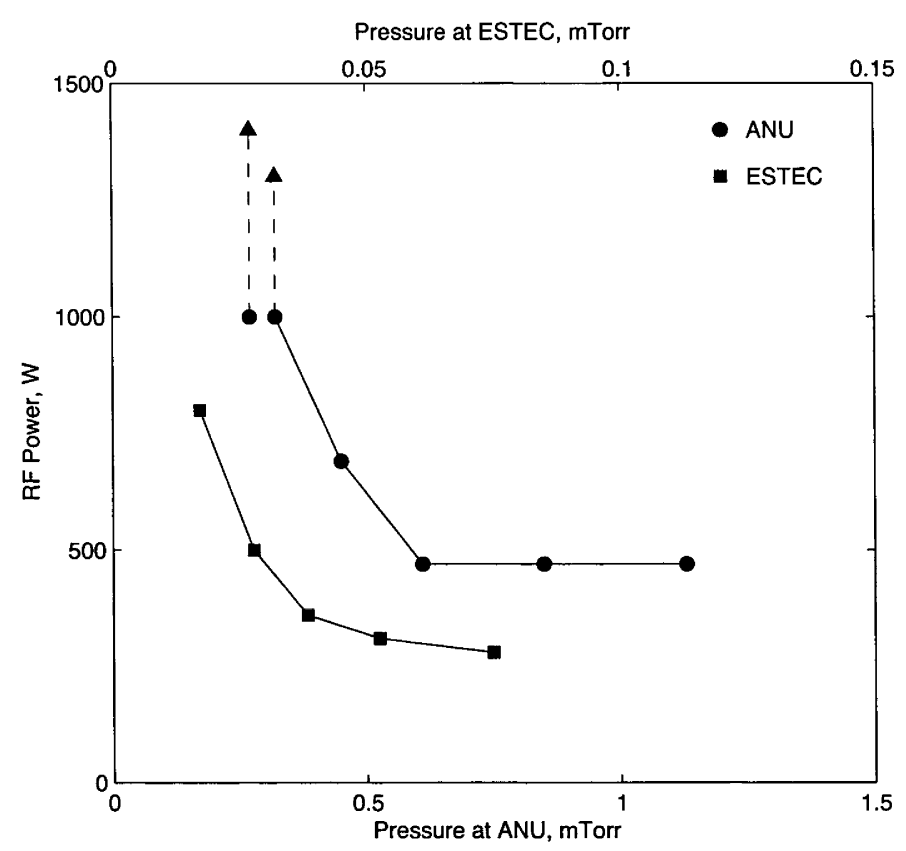

Figure 6.1: The RF power at which the transition to the high density mode occurs as a function of the operating pressure for experiments conducted at ANU (•) and during the ESTEC testing campaign ( $\square$ ). The arrows indicate the RF powers that are likely to be required for the transition to occur at these lower pressures.

argon at a pressure of $2 \mathrm{mTorr}$ when the magnetic field was reduced to approximately $50 \mathrm{G}$ by reducing the current applied to each solenoid to $1 \mathrm{~A}$. Since the high density mode in argon can not be produced at the standard magnetic field and pressure conditions investigated in Chapter 4, no further experiments with argon will be discussed in this thesis and experiments will focus on characterising the blue mode found with xenon.

\subsection{Source Characterisation}

In order to characterise the high density mode found in xenon, measurements were made using the Langmuir probe positioned inside the HDLT source tube at various operating powers and pressures. At an operating pressure of $0.45 \mathrm{mTorr}$ and with the Langmuir probe positioned on the centreline $8 \mathrm{~cm}$ inside the source at $\mathrm{z}=-5 \mathrm{~cm}$ and $\mathrm{r}=0 \mathrm{~cm}$, the density was measured as the RF power was increased. As shown in Figure 6.2, the plasma density in the source increases as the RF power is increased until a dramatic increase in the plasma density occurs at about $625 \mathrm{~W}$. The plasma density in the source then continues to increase with 


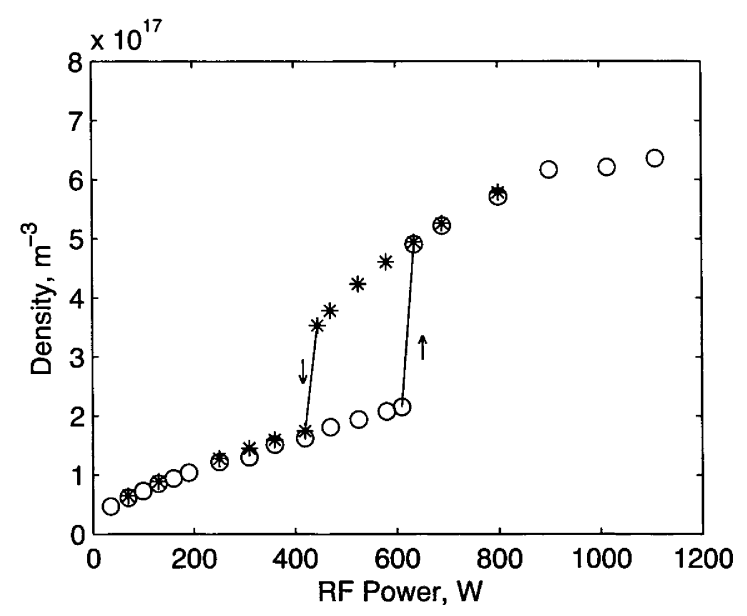

Figure 6.2: The plasma density at $\mathrm{z}=-5 \mathrm{~cm}, \mathrm{r}=0 \mathrm{~cm}$ as the $\mathrm{RF}$ power is increased $(\mathrm{O})$ and as the RF power is decreased (*) at $0.45 \mathrm{~m}$ Torr with the hysteresis marked by the solid line and arrows.

increasing RF power, reaching a maximum of approximately $6.3 \times 10^{17} \mathrm{~m}^{-3}$ at $1110 \mathrm{~W}$ for these operating conditions. At an operating pressure of $0.45 \mathrm{mTorr}$ this represents an ionisation degree of approximately 0.045 . Over the region of the transition, the plasma density inside the source increases by 2.3 times from approximately $2.1 \times 10^{17} \mathrm{~m}^{-3}$ at $610 \mathrm{~W}$ to approximately $4.9 \times 10^{17} \mathrm{~m}^{-3}$ at $635 \mathrm{~W}$ for these operating conditions. This corresponds to an increase in the degree of ionisation from approximately 0.015 to approximately 0.038 over a range of only $25 \mathrm{~W}$ at this location inside the source, which is a significant improvement.

The transition here occurs at similar RF power to that observed during the tests of the HDLT prototype at ESTEC (Charles et al., 2008). The density measured in the source is an order of magnitude lower than that which was estimated in the source during the ESTEC tests, however the operating pressure was significantly lower in that instance $(0.015$ mTorr to 0.15 mTorr at ESTEC compared to $0.45 \mathrm{mTorr}$ here). Few measurements of mode transitions in xenon are reported in the literature, although recent measurements in the HELIX experiment with xenon have shown density increases at $550 \mathrm{~W}$ and $750 \mathrm{~W}$, determined from laser induced fluorescence measurements (Biloiu et al., 2009; Biloiu, 2009a). At $750 \mathrm{~W}$ the plasma became a bright white/blue colour and the change in density at $550 \mathrm{~W}$ were attributed to a E-H mode transition and the change at $750 \mathrm{~W}$ to a $\mathrm{H}-\mathrm{W}$ mode transition (Biloiu, 2009b).

Figure 6.2 also shows the plasma density at the same location as the RF power is decreased, starting at a power level with the high density mode established $(800 \mathrm{~W})$. In this case, the high density mode can be maintained at lower powers 
and the transition out of the mode occurs at about $430 \mathrm{~W}$, demonstrating that significant hysteresis is present. Importantly, as the transition out of the high density mode occurs the matching of the plasma also changes and the parallel capacitor, $C_{2}$, must be returned to its previous value when in the low density mode.

With the Langmuir probe still located on the centreline $8 \mathrm{~cm}$ inside the source at $\mathrm{z}=-5 \mathrm{~cm}$, the DC component of the floating potential, $V_{f_{D C^{\prime}}}$ and the RF component of the floating potential, $V_{f_{R F}}$, were measured as a function of RF power at 0.45 mTorr. Figure 6.3a shows that as the RF power is increased, the floating potential decreases, becoming more negative. At the transition to the high density mode (marked with the dashed line), $V_{f_{R F}}$ increases significantly and then as the $\mathrm{RF}$ power is increased further it decreases.

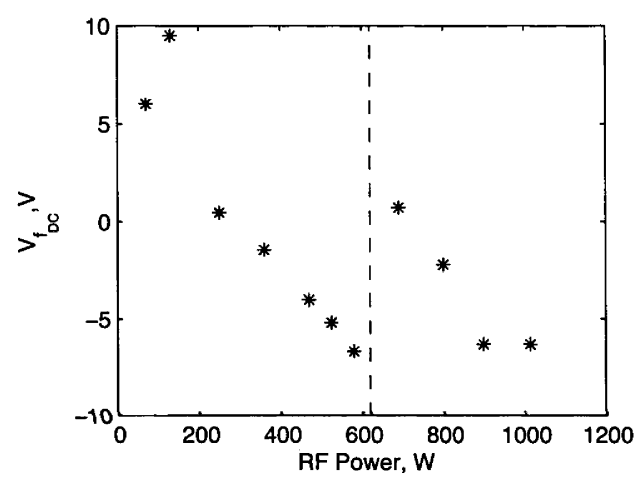

(a)

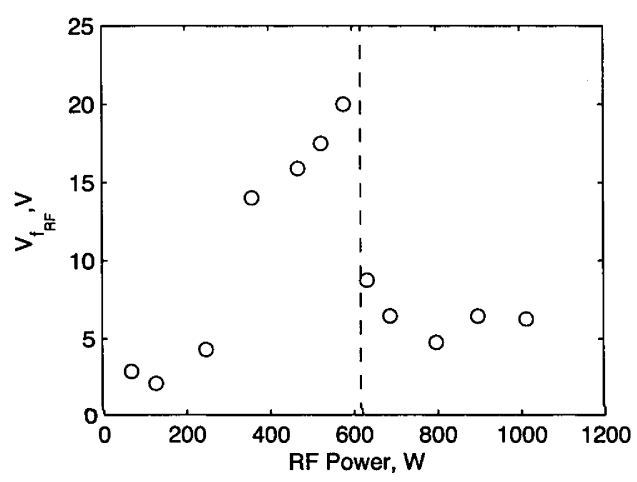

(b)

Figure 6.3: The DC component of the floating potential, $V_{f_{D C}}$ (a) and the RF component of the floating potential, $V_{f_{R F}}$ measured on the Langmuir probe (b) at $\mathrm{z}=-5 \mathrm{~cm}$, $\mathrm{r}=0 \mathrm{~cm}$ versus $\mathrm{RF}$ power at $0.45 \mathrm{mTorr}$. The dashed line marks the RF power at which the transition occurs.

Figure 6.3b shows the change in $V_{f_{R F}}$ as the RF power is increased. The variation of $V_{f_{R F}}$ with RF power gives an indication of the variation of the RF component of the plasma potential, $V_{p_{R F}}$ as a function of RF power (Charles et al., 2000). At the transition to the high density mode $V_{f_{R F}}$ decreases significantly, indicating that the RF modulation in the plasma has reduced and that the capacitive coupling has been reduced in favour of the inductive coupling. Hence, more of the RF power goes into increasing the plasma density, which is consistent with the density measurements discussed earlier. Other researchers have reported similar changes in both the DC and RF components of the floating potential in helicon sources operating in argon when transitioning from the capacitive to helicon wave mode (Perry et al., 2002; Longmier and Hershkowitz, 2008). 


\subsection{Pressure Dependance}

Measurements of the density inside the source were also made at various operating pressures to investigate whether the mode transition scales with pressure. The Langmuir probe was again positioned on the centreline $8 \mathrm{~cm}$ inside the source at $\mathrm{z}=-5 \mathrm{~cm}$ and $\mathrm{r}=0 \mathrm{~cm}$ and the density measured as the RF power was increased. The pressure was varied from 0.27 mTorr to $1.13 \mathrm{mTorr}$. As shown in Figure 6.4, the transition to the high density mode occurs consistently at $470 \mathrm{~W}$ for pressures greater than 0.61 mTorr. No transition to a high density mode occurs at pressures below 0.45 mTorr. Below 0.45 mTorr a $n \propto \sqrt{P_{R F}}$ trend can be fitted to the plasma density data, as shown as a solid line in Figure 6.4. It is clear that, for pressures below 0.45 mTorr at all RF powers and at low powers for pressures greater than $0.45 \mathrm{mTorr}$, the plasma density increases proportionally to the square root of the RF power. This is clear evidence that the low density mode is a capacitively coupled mode.

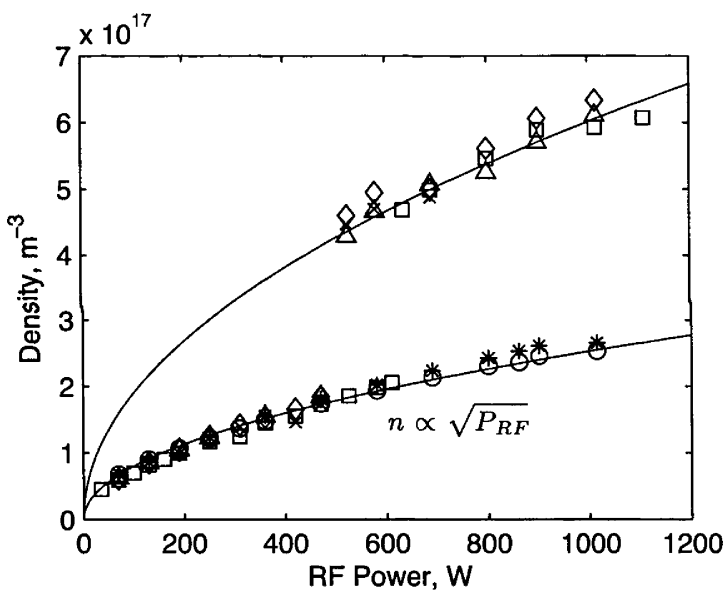

Figure 6.4: The plasma density at $\mathrm{z}=-5 \mathrm{~cm}, \mathrm{r}=0 \mathrm{~cm}$ versus $\mathrm{RF}$ power at various pressures. The solid lines show fits where the density is proportional to the square root of the RF power. Measurements are made at $0.27 \mathrm{mTorr}(\bigcirc), 0.32 \mathrm{mTorr}(*), 0.45 \mathrm{mTorr}$ $(\square), 0.61 \mathrm{mTorr}(\triangle), 0.85 \mathrm{mTorr}(\diamond)$ and $1.13 \mathrm{mTorr}(\times)$.

For those pressures where the high density mode does occur, both a $n \propto P_{R F}$ trend and a $n \propto \sqrt{P_{R F}}$ trend could be fitted to the density results for this mode. The plasma could also be in a wave-sustained mode. Irrespective of the mode, the density is not increasing with RF power as much as expected. This could be the result of an increase in the volume of the downstream plasma in the vacuum chamber or a change in the volume of the plasma inside the source. Alternatively, the density may not be has high because energy is being lost via the formation of parasitic plasma around the antenna or by ion acceleration towards the very 
negatively biased antenna, which results in sputtering of the antenna silver plating. The latter two options are unlikely because less secondary discharges are observed around the antenna when in the high density mode. Also, the quality of the matching and stability of the plasma improves in this mode (as discussed later). The rate of silver sputtering was observed to be less when operating in the high density mode when compared to operating at RF powers just prior to the transition where $V_{f_{R F}}$ is considerably higher. These observations suggests that the plasma volume is increasing, either downstream or inside the source. To investigate this, radial measurements were made inside the source at various $R F$ powers.

\subsection{Radial Profile Inside HDLT Source}

The radial density profile inside the source can indicate if the plasma is in the capacitive, inductive or wave-sustained mode. With the Langmuir probe installed via a side port on the vacuum chamber, radial measurements were made inside the source at $\mathrm{z}=-0.5 \mathrm{~cm}$ at various RF powers. The operating pressure was maintained at 0.45 mTorr. Figure 6.5 shows that for RF powers of $70 \mathrm{~W}, 130 \mathrm{~W}$ and $470 \mathrm{~W}$, that is in the capacitive mode, the radial profile is very uniform and is consistent with previous measurements in capacitively coupled plasmas (Franck et al., 2003).

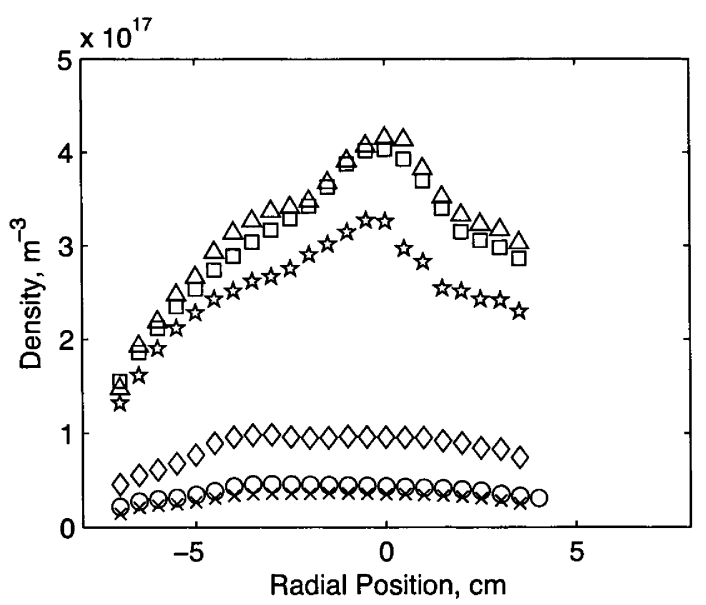

Figure 6.5: The density versus radial position inside the source at $\mathrm{z}=-0.5 \mathrm{~cm}$ at $0.45 \mathrm{~m}$ Torr and a RF power of $70 \mathrm{~W}(\times), 130 \mathrm{~W}(\bigcirc), 470 \mathrm{~W}(\diamond), 635 \mathrm{~W}(\star), 800 \mathrm{~W}(\square)$ and $900 \mathrm{~W}(\triangle)$.

At RF powers beyond the transition to the high density mode, that is at $635 \mathrm{~W}$, $800 \mathrm{~W}$ and $900 \mathrm{~W}$, a central maximum appears in the density profile. Similar 
central peaks have been reported elsewhere and attributed to the propagation of helicon waves (Boswell, 1984; Degeling et al., 1996; Franck et al., 2003; Niemi and Krämer, 2008). Although, this result suggests that the high density mode is a wave-sustained mode it is not conclusive and further measurements with a magnetic fluctuation (B-dot) probe, for example, are required to confirm if helicon waves are present. Such measurements should be pursued in future studies.

Interestingly, increasing the RF power from $800 \mathrm{~W}$ to $900 \mathrm{~W}$ does not result in a change in either the shape or magnitude of the radial density profile and the density appears to saturate. This is consistent with the earlier measurements at various pressures in the high density mode, where the density does not increase as much as anticipated. If the high density mode is a wave-sustained helicon mode, the absence of a proportional increase in density with increasing RF power could be explained by the antenna coupling with the transverse magnetic field of the helicon mode becoming increasing inefficient (Lieberman and Litchtenberg, 2005).

\subsection{Investigation of Matching Box Circuit}

Investigating the electrical characteristics of the matching box can provide some insights into the nature of plasma. Therefore measurements were made at different positions on the matching box using the high voltage probe while the RF power was increased. The operating pressure was maintained at $0.45 \mathrm{mTorr}$ and $V_{p-p}$ was measured. Figure 6.6 shows $V_{p-p}$ at the locations on the matching box shown earlier in Figure 5.6.

The peak-to-peak voltage at position (1) increases linearly with RF power as is expected if the plasma is tuned correctly to the $50 \Omega$ output impedance on the RF generator. The peak-to-peak voltages at positions (2) and (3) will change in response to the plasma properties. Figures $6.6 \mathrm{a}$ and $6.6 \mathrm{~b}$ show that the voltage at both of these locations decrease as the plasma transitions into the high density mode and then continues to increase as the RF power is increased further. The change at position (2), shown in Figure 6.6b, is particularly large $(\backsim 1.5 \mathrm{kV})$.

The quality factor, $Q$, is a measure of the quality of a resonant circuit and is the ratio of the power stored to the power dissipated in the circuit reactance and resistance. In this case, it can be expressed as

$$
Q=\frac{P_{\text {stored }}}{P_{\text {dissipated }}}=\frac{V_{p-p @ 2}}{V_{p-p @ 1}}
$$




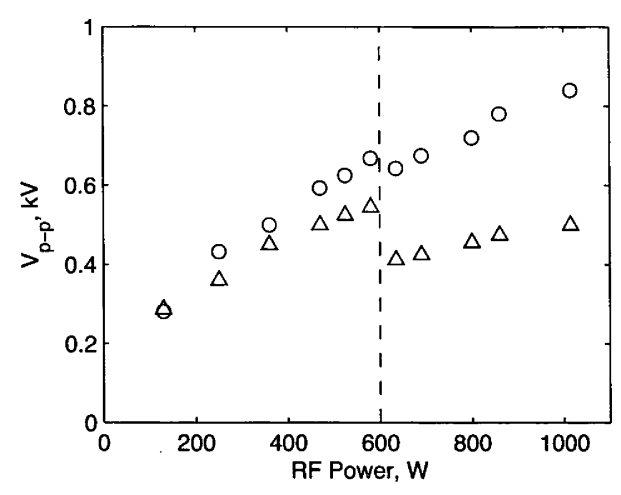

(a)

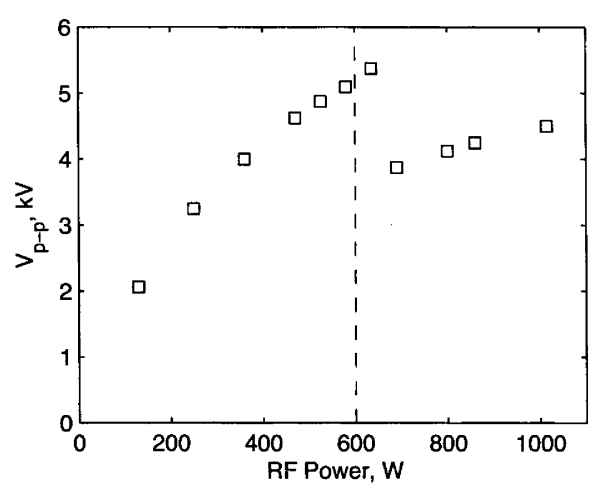

(b)

Figure 6.6: The peak to peak voltage measured (a) at position (1) $(\bigcirc)$ and position (3) $(\triangle)$ and (b) at position (2) ( $\square$ ) in the matching box versus RF power when operating at $0.45 \mathrm{~m}$ Torr. The dashed line marks the RF power at which the transition occurs.

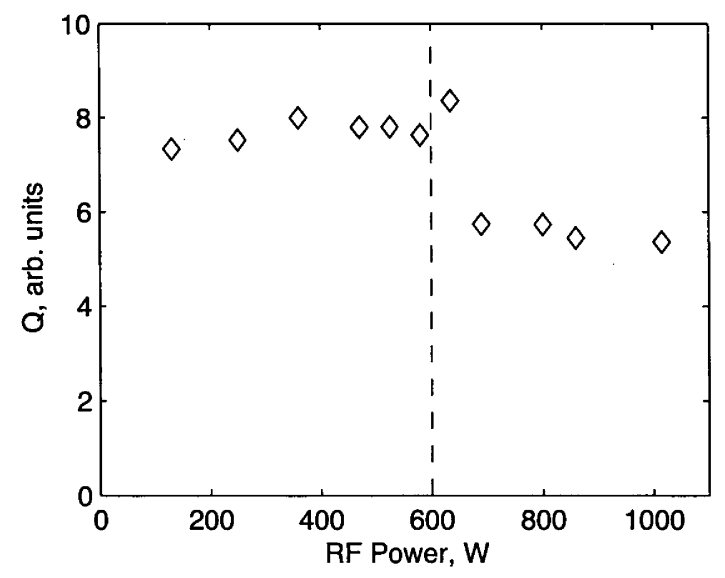

Figure 6.7: The quality factor of the matching box circuit versus RF power when operating at $0.45 \mathrm{~m}$ Torr. 
where $V_{p-p @ 2}$ and $V_{p-p @ 1}$ are the peak-to-peak voltages measured at positions (2) and (1) on the matching box circuit. Using these values, the $Q$ was computed and is shown in Figure 6.7 as a function of RF power. The $Q$ drops from about 7 to 5.5 following the transition into the high density mode. A plasma with a low $(Q<10)$ quality factor is easy to match and is more stable than a plasma with a higher $Q$ (Rayner et al., 1996). This is consistent with the results here as the plasma is easier to match once in the high density mode. The decrease in $Q$ when in the high density mode also indicates that the effective plasma resistance on the antenna is increasing and that more of the RF power is being deposited into the plasma. This is consistent with the measured increase in the plasma density in this mode and the work of other researchers (Perry et al., 1991).

\subsection{Downstream Measurements}

Using the Langmuir probe installed axially, the plasma density in the exhaust of the HDLT prototype was measured on the chamber centreline for two cases. Figure 6.8 shows the density (on a logarithmic scale) in the low density mode at $470 \mathrm{~W}(\square)$ and in the high density mode at $800 \mathrm{~W}(\bigcirc)$ from inside the source to approximately $50 \mathrm{~cm}$ downstream. It is clear that the plasma density when in the high density mode is higher not only in the source but also in the downstream region compared to the low density mode. In the downstream region beyond $\mathrm{z}=10 \mathrm{~cm}$ a linear trend line can be fitted to the results for both cases shown in Figure 6.8. The plasma density is therefore decaying exponentially over this region which indicates that there is a Boltzmann expansion downstream. Figure 6.8 also indicates that the measurements in the source at $\mathrm{z}=-0.5 \mathrm{~cm}$ described earlier are downstream of the heating region, that is downstream from where the plasma is generated.

Axial measurements were made in the high density mode with the RFEA in order to measure the plasma potential and determine the presence of an ion beam. The operating pressure was maintained at $0.45 \mathrm{mTorr}$ and the RF power at $800 \mathrm{~W}$ while the RFEA was moved from $4 \mathrm{~cm}$ inside the source at $\mathrm{z}=-1 \mathrm{~cm}$ to a region far downstream at $\mathrm{z}=53 \mathrm{~cm}$. At this stage, the axial IEDFs showed no evidence of an ion beam, although more detailed investigations in the future may find evidence of an ion beam in the high density mode.

Irrespective, the plasma potential is an important parameter and the axial potential profile is shown in Figure 6.9. It shows that the plasma potential in the source is high at about $60 \mathrm{~V}$ and reduces to around $40 \mathrm{~V}$ a few centimetres downstream 


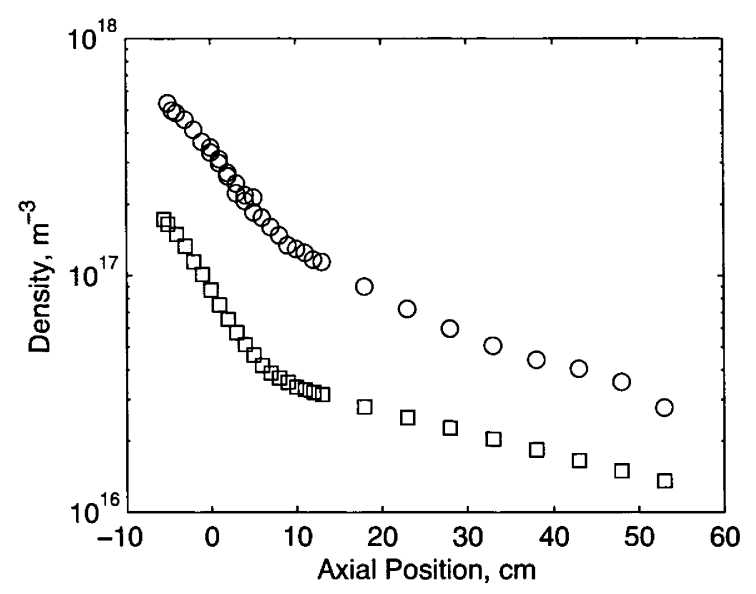

Figure 6.8: The density at $\mathrm{r}=0 \mathrm{~cm}$ versus axial position at $470 \mathrm{~W}(\square)$ and in the high density mode at $800 \mathrm{~W}(\mathrm{O})$ and 0.45 mTorr.

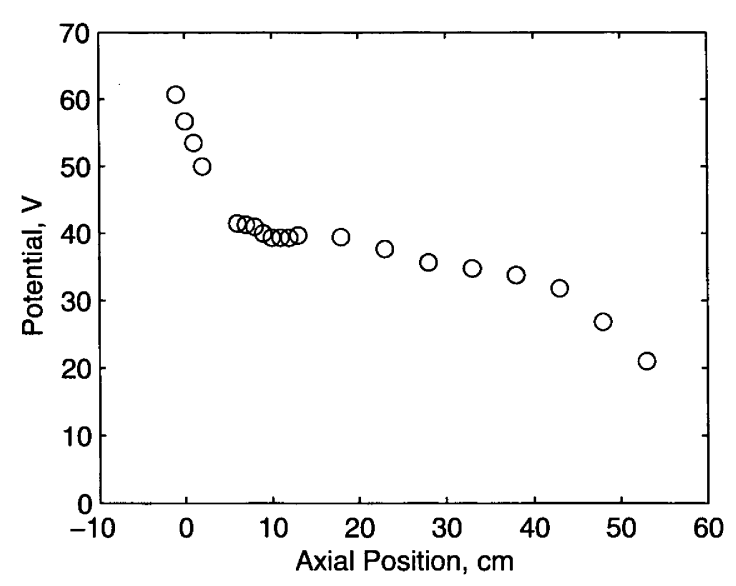

Figure 6.9: The plasma potential at $\mathrm{r}=-0 \mathrm{~cm}$ versus axial position in the high density mode at $800 \mathrm{~W}$ and $0.45 \mathrm{mTorr}$. 
of the exit of the HDLT source tube. The plasma potential then continues to decay downstream as the plasma expands.

Using the axial density and potential profiles for the high density $800 \mathrm{~W}$ case, the electron temperature in the downstream region can be determined using the Boltzmann relation, as described earlier in Section 4.2.3. Figure 6.10 shows a logarithmic plot of the plasma density measured with the Langmuir probe versus the local plasma potential measured with the RFEA in the region downstream of the HDLT source in the high density mode at $800 \mathrm{~W}$ and 0.45 mTorr. The data in this region fits very well to a straight line, where the slope yields an electron temperature of $5.7 \mathrm{eV}$ (from Equation 4.3). This result confirms that downstream of the HDLT source the plasma follows a Boltzmann expansion.

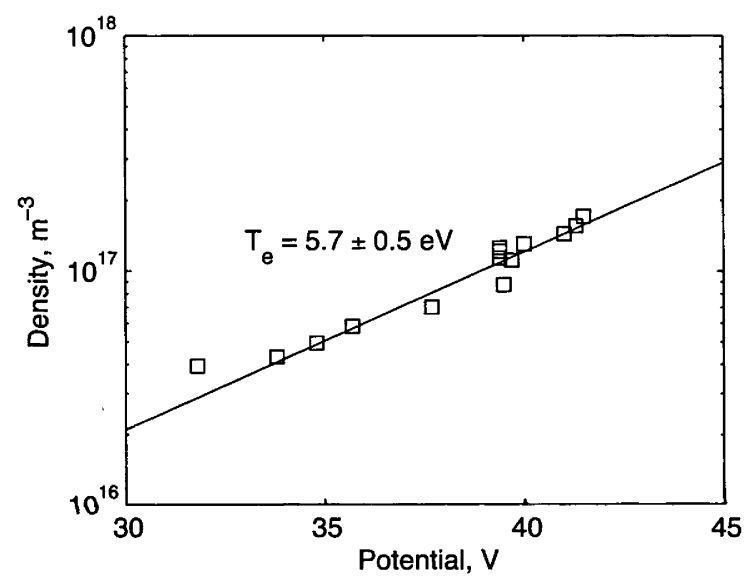

Figure 6.10: The plasma density versus the plasma potential measured downstream in the high density mode at $800 \mathrm{~W}$ and $0.45 \mathrm{mTorr}$.

The tail of the electron energy distribution function (EEDF) downstream was also obtained for the high density mode using the RFEA in the electron collection mode. This was achieved by reversing the polarity on the grids of the RFEA. More details on this method can be found elsewhere (Charles, 1993; Conway et al., 1998). The measurement was made downstream with the RFEA at $\mathrm{z}=15 \mathrm{~cm}, \mathrm{r}=0 \mathrm{~cm}$ and operating the plasma at $800 \mathrm{~W}$ and $0.45 \mathrm{mTorr}$.

Figure 6.11 shows the natural logarithm of the electron current versus the voltage applied to the discriminator of the RFEA for these operating conditions. $V_{d}=0 \mathrm{~V}$ corresponds to the plasma potential at the front of the sheath around the probe (about $40 \mathrm{~V}$ at this location). By plotting a straight line to the data and taking the gradient of the line, the electron temperature is found to be about $6 \mathrm{eV}$ (Ingram and Braithwaite, 1988; Charles, 1993; Gahan et al., 2008). This result is in good agreement with the temperature deduced from the Boltzmann relation de- 
scribed earlier and with downstream measurements $(5.5 \mathrm{eV})$ taken with an RF compensated probe in a similar system (Takahashi et al., 2007).

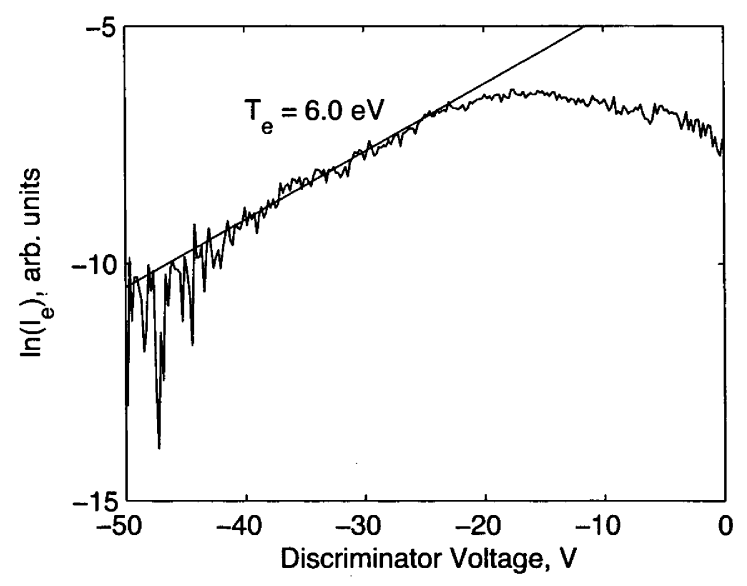

Figure 6.11: The natural logarithm of the electron current measured with the RFEA located at $\mathrm{z}=15 \mathrm{~cm}, \mathrm{r}=0 \mathrm{~cm}$ in the high density mode at $800 \mathrm{~W}$ and $0.45 \mathrm{mTorr}$.

\subsection{MFMI Measurements with Xenon at High Power}

Using the MFMI described in Chapter 3, measurements were made of the force imparted by the thruster exhaust when increasing the RF power with xenon. Here the measurements were made using the MFMI with the $50 \mathrm{~mm}$ target installed. Figure 6.12 shows the force from the plasma measured by the MFMI, $F_{p}$, as a function of the RF power when operating the HDLT prototype with $10 \mathrm{sccm}$ of xenon at $0.45 \mathrm{mTorr}$ and an axial maximum magnetic field of $138 \mathrm{G}$. For reference, the force from the neutral propellant flow equals $14.0 \mu \mathrm{N}$ at this pressure with xenon. As the RF power is increased, $F_{p}$ increases until a large increase in $F_{p}$ occurs at $580 \mathrm{~W}$. At this RF power the plasma transitions from the capacitive mode to the high density mode. The error in the $F_{p}$ measurement in the high density mode increases compared to when operating in the capacitive mode. This is because when the shutter is retracted, larger oscillations of the MFMI target occur as the initial impulse from the impacting plasma is larger.

Using the Langmuir probe positioned at $\mathrm{z}=12 \mathrm{~cm}$ and at $\mathrm{z}=14 \mathrm{~cm}$, the density was measured in front and behind the MFMI target as a function of RF power, as shown in Figure 6.13. At the transition to the high density mode, the density on both the front and the back sides of the target increases considerably, as expected. The radial density profile was also measured in front and behind the MFMI target 


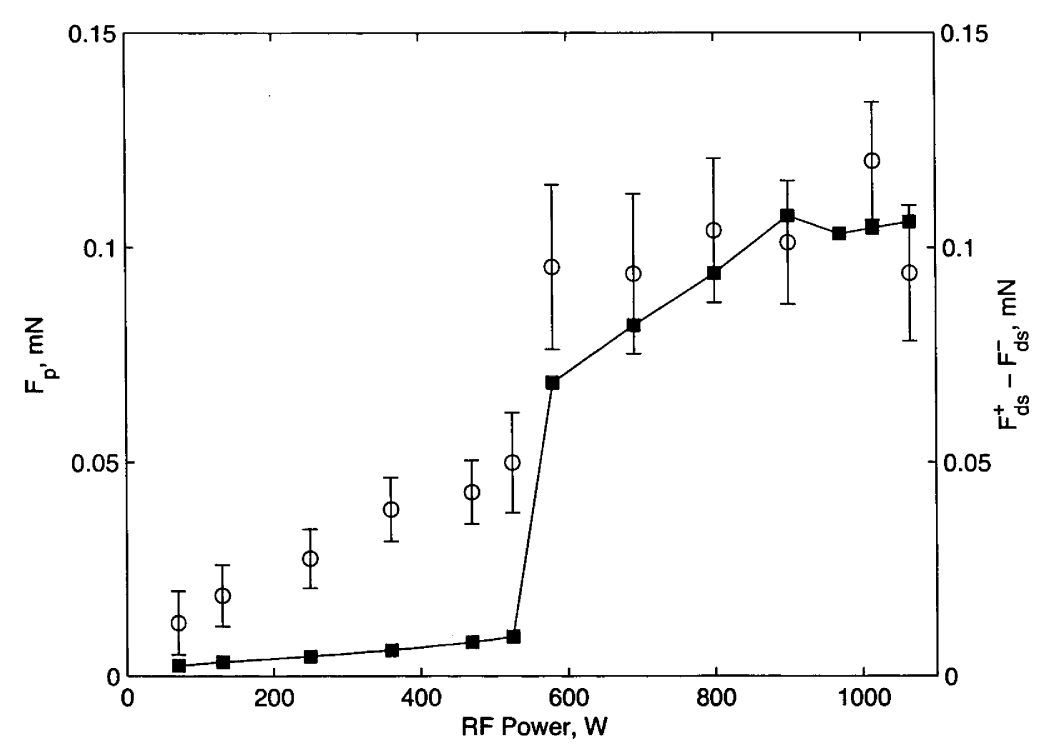

Figure 6.12: The force from the plasma $(\bigcirc)$ measured by the MFMI with the $50 \mathrm{~mm}$ diameter target for a xenon plasma formed at $0.45 \mathrm{mTorr}$ and $138 \mathrm{G}$ as a function of $\mathrm{RF}$ power. The calculated force from the downstream plasma, $F_{d s}^{+}-F_{d s^{\prime}}^{-}(\boldsymbol{\square})$ as a function of RF power based on the measurements with the Langmuir probe is also shown.

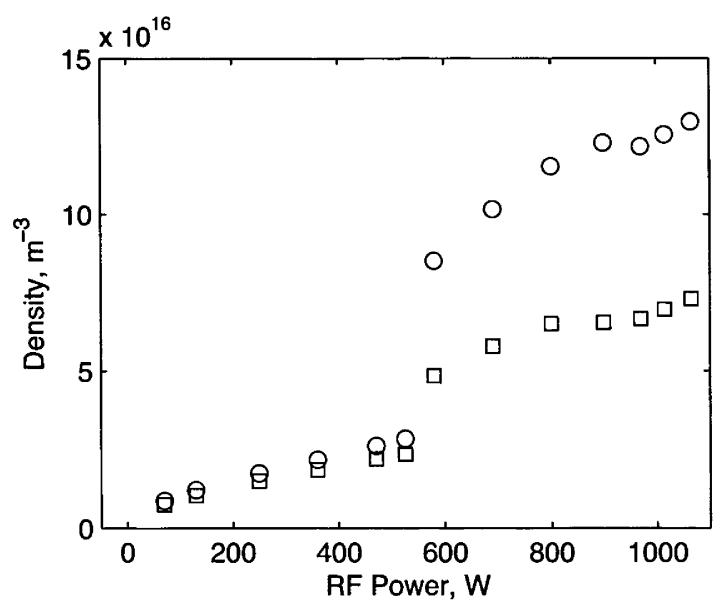

Figure 6.13: The plasma density measured with the Langmuir probe positioned in front of the MFMI target at $\mathrm{z}=12 \mathrm{~cm}(\bigcirc)$ and behind the MFMI target at $\mathrm{z}=14 \mathrm{~cm}(\square)$ as a function of RF power. 
at $130 \mathrm{~W}, 470 \mathrm{~W}$ and $800 \mathrm{~W}$. At $130 \mathrm{~W}$ and $470 \mathrm{~W}$, the radial profiles on both sides of the target are very flat and almost equal, consistent with a capacitively coupled plasma (Franck et al., 2003).

Figure 6.14 shows the normalised radial density profiles when operating at $800 \mathrm{~W}$. It is clear that a wake forms behind the target, which is strong evidence for a flowing plasma, and that the density behind the MFMI target for this case is constant over the target area. Although the same is not true for the density profile on the front of the target, the density varies by less than $10 \%$ over the diameter. Therefore, the radial density profile in both the capacitive and the high density mode, at this position downstream, are assumed constant across the radius of the MFMI target. Subsequently, the area of the plasma on the front and back sides of the target, $A_{p}^{+}$and $A_{p}^{-}$respectively, can be calculated assuming a radius of $2.5 \mathrm{~cm}$.

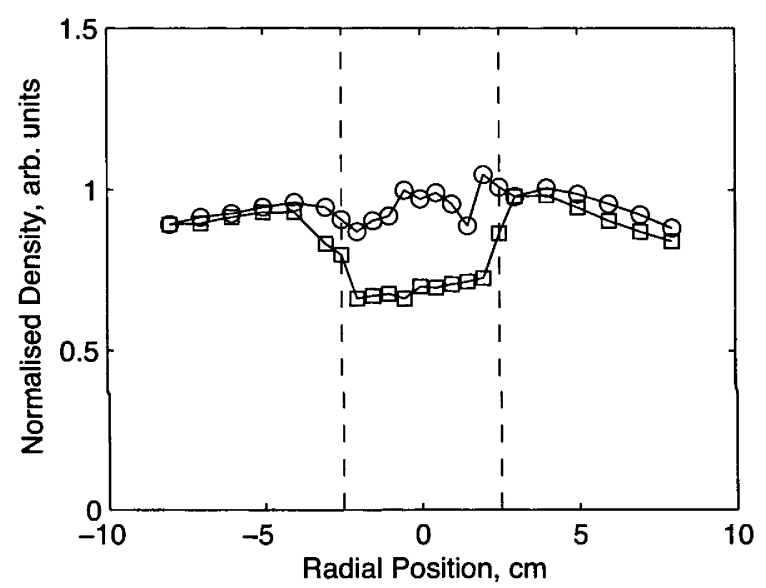

Figure 6.14: The normalised radial plasma density profile measured with the Langmuir probe positioned in front of the MFMI target at $z=12 \mathrm{~cm}(\bigcirc)$ and behind the MFMI target at $\mathrm{z}=14 \mathrm{~cm}(\square)$ when operating at $800 \mathrm{~W}$. The dashed lines mark the edges of the target plate.

Given the above information and assumptions, the force from the downstream plasma, $F_{d s}^{+}-F_{d s}^{-}$, can be calculated using Equation 3.22 and compared to the total force from the plasma, $F_{p}$, measured with the MFMI. $v_{B}=2.09 \times 10^{3} \mathrm{~ms}^{-1}$ when the electron temperature measured downstream earlier is used. Figure 6.12 also shows the calculated $F_{d s}^{+}-F_{d s}^{-}$as a function of RF power and it is clear that, when operating in the high density mode, the calculated value is in good agreement with that measured with the MFMI. In this region ( $>580 \mathrm{~W})$, no ion beam is detected in front of the MFMI target with the RFEA and therefore the force measured at this position is influenced entirely by the plasma density. 
At low powers in the capacitively coupled mode, the measured $F_{p}$ is higher than the calculated $F_{d s}^{+}-F_{d s}^{-}$. Previous RFEA measurements (Section 4.10) confirm that an ion beam is present at this downstream position for these conditions, consistent with experiments in a similar experiment (Charles et al., 2006). Detailed measurements of the ion beam properties for these lower power conditions have not been undertaken here and should be pursued in future studies. At low powers in the capacitive mode the difference in the force measured by the MFMI at $z=13 \mathrm{~cm}$ and that calculated from the difference in the plasma densities at the front and back of the MFMI target could be because of the presence of the ion beam mentioned above. Alternatively, fast neutrals generated by charge exchange collisions could also contribute to the increase in force measured by the MFMI since the relative ionisation is lower at these powers and more neutral xenon may be present. Determining the velocity and density of these neutrals, however, is far from trivial and is beyond the scope of this work. Irrespective, in the high density mode the momentum flux is clearly dictated by the increase in the plasma density. The results obtained here indicate that qualitatively the thrust produced by the HDLT prototype exhaust increases with the applied RF power when operating with xenon.

\subsection{Estimate of Thrust}

From the characterisation of the high density mode in xenon described in this chapter, it is possible to estimate thrust produced by the HDLT prototype when operating in this mode. The same assumptions outlined in Section 4.11 apply when considering the idealised case, that is, when the HDLT prototype is operating in space. Using Equation $4.8, T_{i}$ can be found when the HDLT prototype is operating at $800 \mathrm{~W}$, at a pressure of $0.45 \mathrm{mTorr}$ and with a magnetic field of $138 \mathrm{G}$. The measured density inside the HDLT source is approximately $5.5 \times 10^{17} \mathrm{~m}^{-3}$, $v_{i_{e x}}=9,390 \mathrm{~ms}^{-1}$ and $v_{B}=2,100 \mathrm{~ms}^{-1}$ and therefore $T_{i} \sim 36.4 \mathrm{mN}$. The thrust estimated for these conditions is approximately 8.5 times greater than the thrust estimated earlier when operating at $130 \mathrm{~W}$ with xenon $(4.2 \mathrm{mN})$. For reference, the force from the neutral propellant at 0.45 mTorr, determined using Equation 1.11, is $T_{n}=0.60 \mathrm{mN}$, which is about $1.5 \%$ of $T_{i}$.

As discussed previously in Section 4.11, the limited pumping speed of the space simulation chamber and the presence of downstream plasma mean that it is not possible to quantitatively compare the force measured with the MFMI when in the high density mode to that calculated for the idealised case. Moreover, when using the MFMI with the $50 \mathrm{~mm}$ target, as is the case here, only a fraction of the 
plasma flux ejected by the HDLT prototype is being measured by the MFMI. Future measurements in a cryogenically pumped space simulation chamber should provide a more realistic measurement of the thrust produced by the HDLT prototype when operating in the high density mode. Irrespective, the qualitative behaviour of the MFMI results presented here are consistent with the behaviour of the plasma in the source.

\subsection{Spectroscopic Studies}

As referred to earlier in Section 6.2, the appearance of the plasma in the source and exhaust region changes from a light purple colour to a bright white/blue colour when the mode transition occurs as the RF power is increased (Figure 6.15). A preliminary spectroscopic study was conducted to investigate this and attempt to quantify the colour changes observed. The intensity of emission is also proportional to the plasma density (Scharer et al., 2002) and therefore spectroscopic techniques could be used in future studies of the plasma produced by the HDLT prototype.

Using the Ocean Optics QE65000 Spectrometer, measurements were made through the upstream window focusing on the closed end of the HDLT source. Spectra were taken at various RF powers when operating with $10 \mathrm{sccm}$ at $0.45 \mathrm{mTorr}$ and a maximum axial magnetic field of $138 \mathrm{G}$. Figures $6.16 \mathrm{a}$ and $6.16 \mathrm{~b}$ compare the intensity in the blue portion of the visible spectrum $(425-525 \mathrm{~nm})$ and the intensity in a portion of the near infrared spectrum $(800-950 \mathrm{~nm})$, respectively, when operating in the capacitively coupled mode at $130 \mathrm{~W}$ (red line) and in the high density 'blue' mode at $800 \mathrm{~W}$ (blue line). As expected, the intensity of several persistent lines for neutral (Xe I) and singly ionised (Xe II) xenon increase in both the blue portion and the near infrared portion of the spectrum. The Xe II lines at $483.3,528.7$ and $541.0 \mathrm{~nm}$ are particularly prominent at $800 \mathrm{~W}$ and probably account for the intense blue colour observed.

The emission intensity of the Xe II line at $528.7 \mathrm{~nm}$ and the Xe I line at $822.7 \mathrm{~nm}$ was investigated as a function of RF power as shown in Figure 6.17. For both cases the intensity (and hence density) increase with RF power with a large increase occurring when the plasma transitions from the capacitive mode to the high density mode (marked by the dashed line). The intensity of the Xe II line at $528.7 \mathrm{~nm}$ increases by $\backsim 12.5$ times between $580 \mathrm{~W}$ and $690 \mathrm{~W}$ while the intensity of the Xe I line at $822.7 \mathrm{~nm}$ only doubles over the same power range. This suggests that the proportion of singly ionised xenon atoms increases significantly 


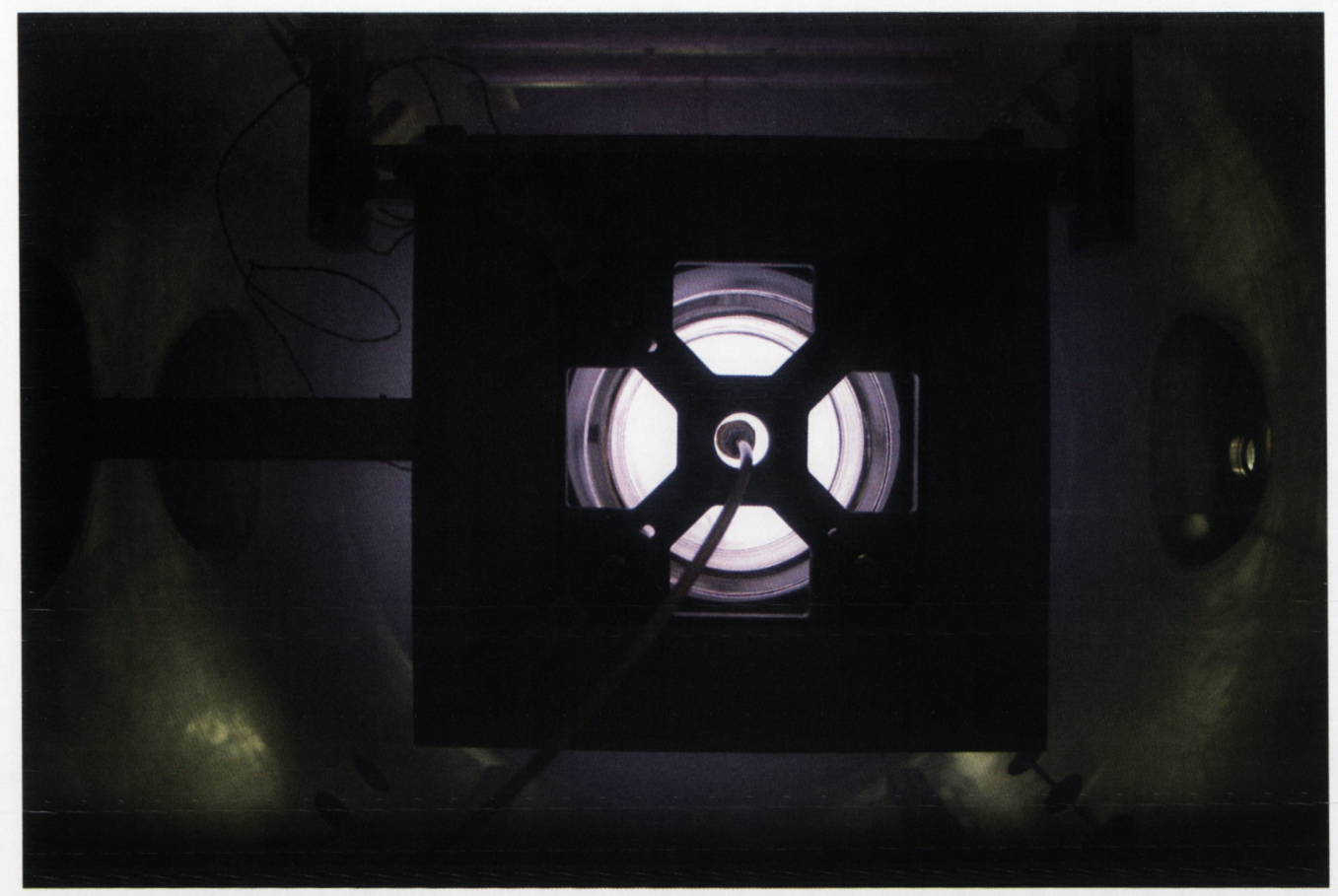

(a)

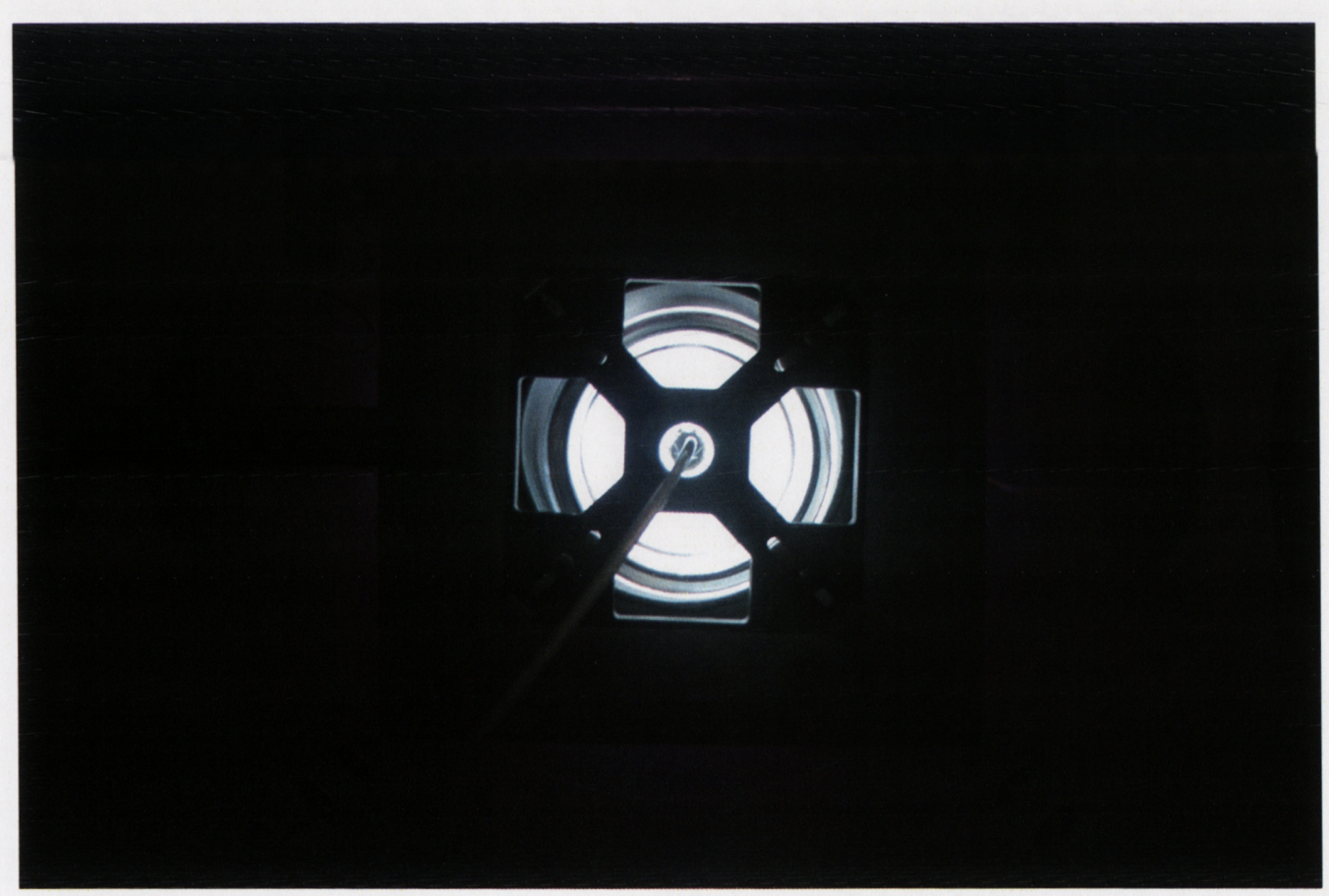

(b)

Figure 6.15: View of the closed end of the HDLT source when operating with xenon at (a) $130 \mathrm{~W}$ in the capacitive mode and (b) $800 \mathrm{~W}$ in the high density 'blue' mode. 


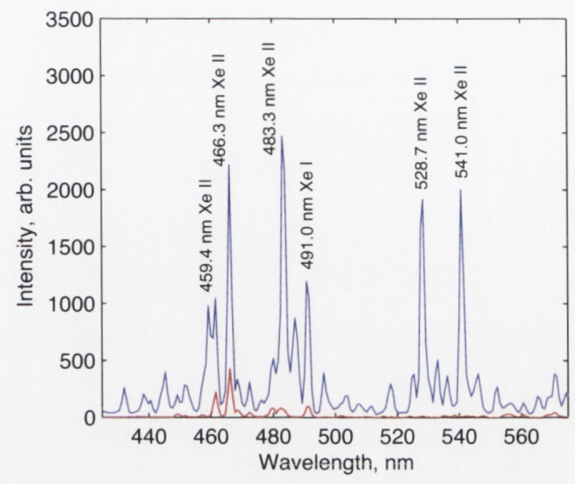

(a)

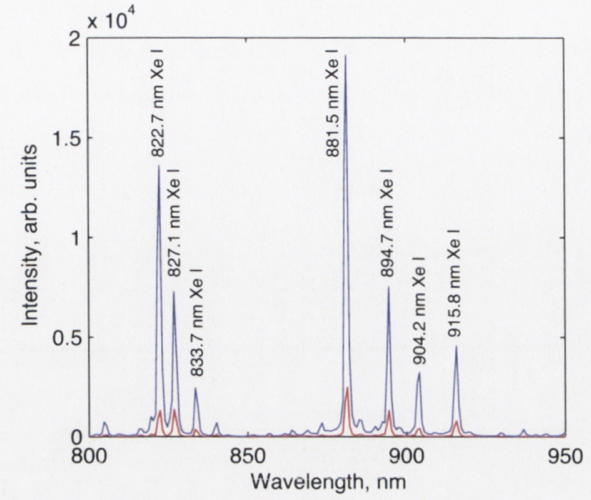

(b)

Figure 6.16: The emission spectrum for xenon in the HDLT source when operating at $130 \mathrm{~W}$ (red line) and $800 \mathrm{~W}$ (blue line). Persistent Xe I and Xe II lines are marked.

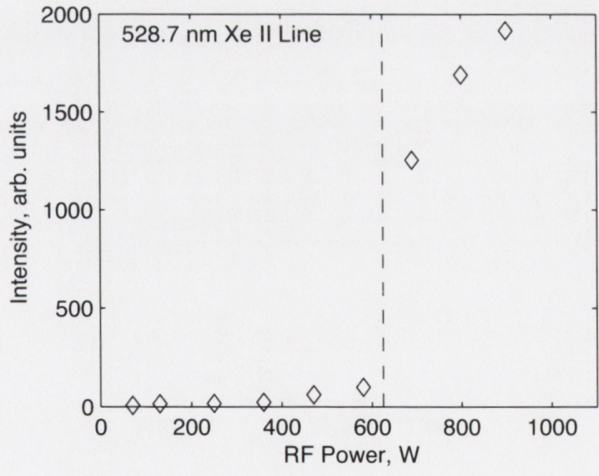

(a)

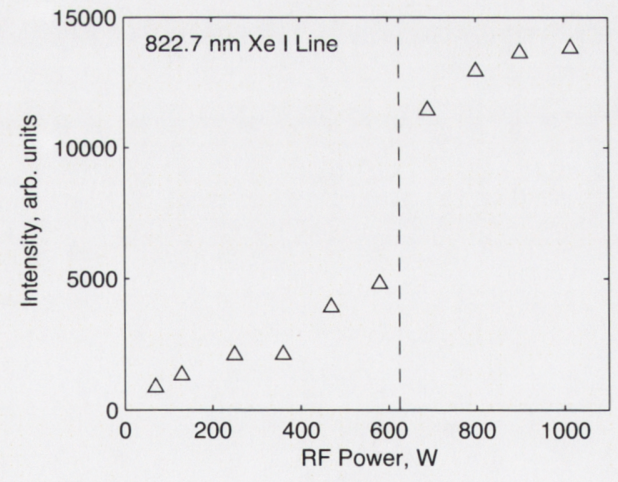

(b)

Figure 6.17: The emission intensity in the HDLT source as a function of the RF power for (a) the Xe II line at $528.7 \mathrm{~nm}$ and (b) the Xe I line at $822.7 \mathrm{~nm}$. The dashed line marks the RF power at which the mode transition occurs. 
as a result of the transition to the high density mode. This result is consistent with the increases in plasma density measured in the source, and discussed in Section 6.3, as the plasma transitions from the capacitively coupled mode to the high density mode.

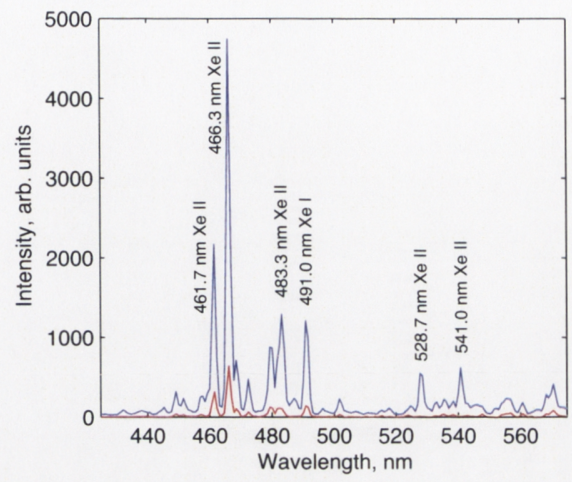

(a)

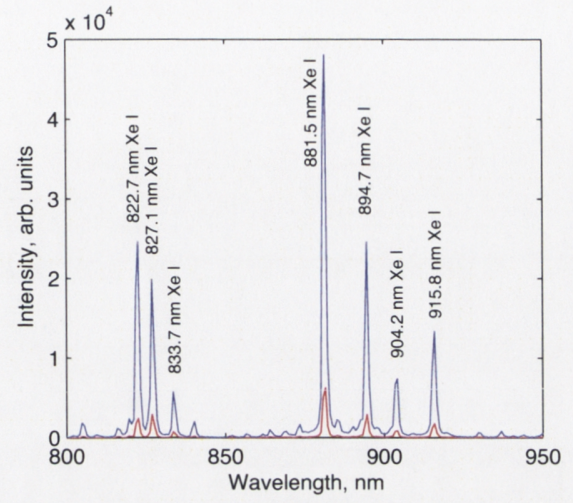

(b)

Figure 6.18: The emission spectra for xenon in the HDLT exhaust when operating at $130 \mathrm{~W}$ (red line) and $800 \mathrm{~W}$ (blue line). Persistent Xe I and Xe II lines are marked.

Measurements were also made for the same operating conditions through a side window that focused the spectrometer at the exhaust region downstream of the HDLT source. The spectra shown in Figures 6.18 and 6.19 are very similar to those obtained when focused up the HDLT source as discussed above. Here, the intensity in the exhaust region of the Xe II line at $528.7 \mathrm{~nm}$ increases by $\backsim 8.5$ times while the intensity of the Xe I line at $822.7 \mathrm{~nm}$ doubles between $580 \mathrm{~W}$ and $690 \mathrm{~W}$, that is, the region over which the mode transition occurs. These results are also consistent with the behaviour of the plasma density measurements made in front of the MFMI target as a function of RF power, which are discussed in Section 6.8.

\subsection{Conclusions}

In this chapter, the high density 'blue' mode in xenon first observed during tests with the HDLT prototype at ESTEC has been reproduced and characterised with various diagnostics. The RF power at which the transition occurs has been found to be a function of the operating pressure and the results are consistent with those obtained during the ESTEC testing campaign. The plasma source has been investigated at various operating RF powers and pressures. At low RF powers, the HDLT prototype operates in the capacitive mode. As the RF power is increased a discrete mode transition occurs and the plasma density increases significantly. 


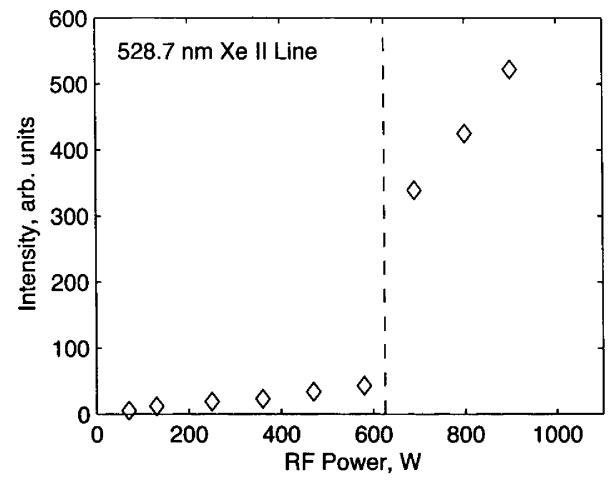

(a)

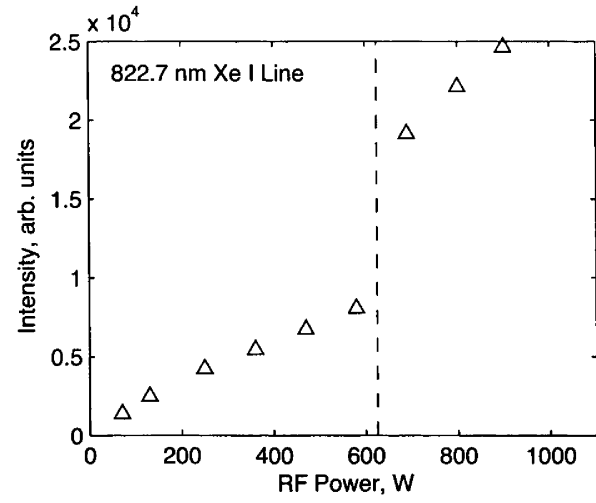

(b)

Figure 6.19: The emission intensity in the HDLT exhaust as a function of the RF power for (a) the Xe II line at $528.7 \mathrm{~nm}$ and (b) the Xe I line at $822.7 \mathrm{~nm}$. The dashed line marks the RF power at which the mode transition occurs.

This mode transition is accompanied by a change in the matching of the plasma and the appearance of bright white/blue plasma inside the source. As the RF power is decreased significant hysteresis is observed. Radial measurements inside the source show a centrally peaked profile in the high density mode. These results have been compared with prior work and suggest that the high density mode may be a wave-sustained helicon mode and therefore a direct E-W mode transition would be present in this case. Future measurements should establish if helicon waves are present via measurements of magnetic fluctuations with a B-dot probe.

Measurements of the electrical characteristics of the matching box have been made and the $Q$ factor shown to decrease once the transition to the helicon mode occurs. This results from an increase in the plasma resistivity consistent with the increased plasma density and the deposition of more RF power into the plasma. The exhaust of the xenon plasma created by the HDLT prototype has been investigated with an RFEA and the MFMI. The IEDFs obtained show no evidence at this stage of an ion beam, however further investigations would be beneficial. The plasma potential has been measured along the central axis of the HDLT source and the vacuum chamber and the plasma downstream has been shown to follow a Boltzmann expansion. The electron temperature in the exhaust has also been determined via two different means and shown to be approximately $6 \mathrm{eV}$.

With the MFMI, configured with the smaller target and positioned $10 \mathrm{~cm}$ downstream of the exit of the HDLT source, measurements of the force produced as a function of the RF power have been made. The density profiles in front and behind the MFMI target have been measured and shows evidence that a wake 
forms behind the MFMI target when in the high density mode. When the plasma transitions from the capacitive mode to the high density mode the momentum flux measured downstream has been found to increase as a result of the increase in the plasma density. This result indicates that the thrust produced by the HDLT prototype can be increased by operating in the high density mode.

The preliminary spectroscopic investigation undertaken here demonstrates that spectroscopic techniques could be used in the future to diagnose and characterise mode transitions in the HDLT prototype and other similar systems. This approach has the advantage of being non-invasive and is very simple to implement. Future tests of the HDLT prototype in a fully cryogenically pumped vacuum chamber, where the operating pressure is lower, are highly recommended. These tests should investigate if an ion beam is present and if such an ion beam can be coupled with the higher densities created in the high density mode. The MFMI could also be used to determine if the ion beam/high density mode combination results in a further increase in the thrust produced by the HDLT prototype. 


\section{Conclusion}

This thesis presents the first detailed tests of the HDLT concept with the helicon source (and the whole thruster) immersed inside a space simulation vacuum chamber. Using an RFEA, the first experimental evidence of a supersonic ion beam forming downstream of a current-free double layer with the helicon source immersed in vacuum has been obtained. At an operating pressure of $0.4 \mathrm{mTorr}$, the ion beam produced by the HDLT prototype has been detected up to $21 \mathrm{~cm}$ downstream and argon ions has been measured at velocities of approximately $8.7 \mathrm{kms}^{-1}$. When operating at lower pressures, the ion beam velocity increases with ion velocities of approximately $11.4 \mathrm{kms}^{-1}$ measured with the RFEA at 0.15 mTorr. Using the RFEA and a Langmuir probe, the plasma produced by the HDLT source has been characterised axially and radially. Downstream the plasma has been shown to follow a Boltzmann expansion with an electron temperature of approximately $5.5 \mathrm{eV}$.

The double layer has been characterised and the influence of the operating pressure and magnetic field on the properties of the plasma have also been investigated. These results have been compared to other experiments and theoretical models. The decay in the ion beam flux axially has been investigated and the ions in the exhaust of the HDLT prototype have been shown to be nonmagnetised, which suggests that ion detachment has occurred. Experiments operating at low power with xenon have demonstrated that a supersonic ion beam can be generated by the HDLT prototype. This is significant as xenon is the propellant of choice for electric propulsion systems.

Following on from the discovery made during the testing campaign at ESTEC, this thesis also presents the first characterisation of the high density 'blue' mode in xenon. This mode occurs when the RF power is increased and the plasma source transitions from the capacitively coupled mode to what may be a helicon 
wave-sustained mode. The transition is accompanied by the appearance of a bright white/blue plasma inside the HDLT source and a significant change in the matching of the plasma. In the 'blue' mode the plasma density inside the HDLT source increases by 2.3 times over the region of the transition. The properties of the plasma in the HDLT source and the exhaust produced downstream have been investigated at various operating powers and pressures. The mode transition has been shown to be a function of the operating pressure, which is consistent with the 'blue' mode observed at ESTEC. A preliminary investigation of the optical emission of the plasma in the source and exhaust region has also been completed via spectroscopy.

The MFMI developed in this thesis is a new plasma diagnostic designed to investigate the thrust imparted by electric propulsion devices. Using techniques developed by the materials science community for surface stress measurements, a laser displacement system has been employed to measure, with a high sensitivity, the displacement of a target plate suspended in a plasma thruster exhaust. Depending upon the diameter of the target plate used, the MFMI is able to measure forces up to $0.5 \mathrm{mN}$ with a resolution of $15 \mu \mathrm{N}$ or forces up to $0.2 \mathrm{mN}$ with a resolution of $5 \mu \mathrm{N}$. This measurement technique is free from $\mathrm{RF}$ interference and thermal effects.

The MFMI has been used here to investigate the plasma exhaust produced by the HDLT prototype. Measurements have been made at various operating pressures, magnetic field configurations and input powers when operating in the low power range with argon. The results obtained are in good agreement with the results calculated from the plasma properties measured at the position of the MFMI with the Langmuir probe and RFEA. MFMI measurements have also been made when using xenon as a function of RF power. The results have provided insights into the nature of the high density 'blue' mode and shown that the thrust produced by the HDLT prototype increases when operating in this mode. Evidence of a wake forming behind the MFMI target when in the high density 'blue' mode was also found.

As a result of the limited pumping speed of the vacuum chamber and the presence of a significant downstream plasma, the thrust levels measured with the MFMI are significantly lower than would be expected if the HDLT prototype was operating in space. Despite this, several qualitative conclusions have be drawn from these results, including that the thrust produced by the HDLT scales with the operating pressure, the inputted RF power and the magnitude of the magnetic field. More significantly, it was found that, when operating with xenon, the transition to the high density 'blue' mode produces a considerable increase in the 
thrust as a result of the increase in the plasma density. At this stage, comparison between this first prototype of the HDLT and flown electric propulsion systems is premature. However, the presented measurements of ion beam velocity, ion beam density and thrust demonstrate that the HDLT is an attractive option for several in space propulsion applications. These positive indicators suggest that the design can be optimised further in the future and additional testing in a fully cryogenically pumped space simulation chamber is necessary to fully characterise and space qualify the HDLT concept.

During the testing of the HDLT prototype inside the space simulation vacuum chamber, several challenges associated with operating the RF antenna in vacuum were identified. Small discharges, known as microarcs, were observed as the RF power applied to the HDLT prototype was increased and the formation of parasitic plasmas downstream and around the RF antenna were observed. To mitigate these problems and enable operation of the HDLT prototype at higher RF powers, several solutions were proposed. By adding a blocking capacitor to the matching box circuit, which floats the antenna, the effects of the microarcing and parasitic plasmas diminished. This thesis is the first investigation and discussion of these issues in the context of ground testing electric propulsion systems.

\subsection{Recommendations and Future Work}

The most obvious recommendation from this thesis is the need to test the HDLT prototype in a space simulation chamber that is fully cryogenically pumped. By significantly increasing the pumping speed of the test chamber, residual gas will be removed faster and less parasitic plasma will form downstream of the HDLT prototype. Although the installation of the blocking capacitor described in Chapter 5 alleviated some of the shortcomings of the experimental setup, a higher pumping speed will enable a test environment that is an even better simulation of space. Reducing the operating pressure downstream has numerous benefits. The force measured by the MFMI will more accurately reflect the thrust produced by the HDLT prototype as the downstream plasma will have less influence on the MFMI results. The electrical efficiency of the HDLT prototype can also be evaluated as less parasitic plasmas will be present and more of the inputted power will be deposited into the plasma in the HDLT source.

During the course of this work, a number of interesting phenomena were observed but not investigated in depth and several interesting questions remain 
unanswered. These may open new avenues for research and have implications for the future operation of the HDLT in space. For example, the MFMI measurements made at various magnetic field strengths revealed a peak in the force produced by the plasma at low magnetic fields. This observation should be investigated further. At this stage, no evidence has been found of an ion beam in the high density 'blue' mode in xenon and it is unknown if the 'blue' mode is a wave-sustained helicon mode. To establish if this is the case, measurements of magnetic fluctuations with a B-dot probe are required. The high density mode in xenon also requires further investigation along with the relationship between the mode transition and the magnitude of the magnetic field. Finally, the results in this thesis have demonstrated that the MFMI is a very useful plasma diagnostic and it could be used in the future to characterise the HDLT prototype further and to investigate the momentum imparted by the exhausts of various plasma thrusters and other diffusive plasmas. 


\section{Bibliography}

Aanesland, A., Charles, C., 2006. Plasma expansion from a dielectric electron cyclotron resonance source. Physica Scripta T122, 19-24.

Aanesland, A., Charles, C., Boswell, R. W., 2005. Grounded radio-frequency electrodes in contact with high density plasmas. Physics of Plasmas 12 (103505), 1-7.

Aanesland, A., Charles, C., Boswell, R. W., Fredriksen, A., 2003. Sputtering effects in a helicon plasma with an additional immersed antenna. Plasma Sources Science and Technology 12, 85-88.

Aanesland, A., Charles, C., Boswell, R. W., Fredriksen, Ȧ., 2004. Helicon plasma with additional immersed antenna. Journal of Physics D: Applied Physics 37, 1334-1341.

Aanesland, A., Meige, A., Chabert, P., 2009. Electric propulsion using ion-ion plasmas. Journal of Physics: Conference Series 162 (012009).

Ahedo, E., Martinez-Sanchez, M., 2008. Theory of a Stationary Current-Free Double Layer in a Collisionless Plasma. Physical Review Letters 103 (135002), 1-4.

Arefiev, A. V., Breizman, B. N., 2005. Magnetohydrodynamic scenario of plasma detachment in a magnetic nozzle. Physics of Plasmas 12 (043504), 1-10.

Au, V., Charles, C., Boswell, R. W., 2006. Interface creation and stress dynamics in plasma-deposited silicon dioxide films. Applied Physics Letters 88 (234103), 1-3.

Baker, G. L., Blackburn, J. A., 2005. The Pendulum: A Case Study in Physics. Oxford University Press, Oxford.

Banetta, S., Falorni, R., Biagioni, L., Saccoccia, G., 2004. Development of a Two-Axis Thrust Stand for Electric Thrusters. In: Proceedings of the 4th International Spacecraft Propulsion Conference. No. ESA SP-55. Cagliari, Sardinia, Italy, pp. 1-6.

Bicker, M., von Hulsen, U., Laudahn, U., Pundt, A., Geyer, U., 1998. Optical deflection setup for stress measurements in thin films. Review of Scientific Instruments 69 (2, Part 1), 460-462.

Biloiu, I. A., 2009a. Laser Induced Fluorescence Studies of Ion Acceleration in Single and Multiple Species Expanding Plasmas. Ph.D. thesis, West Virginia University. 
Biloiu, I. A., 2009b. Private communication.

Biloiu, I. A., Scime, E. E., Biloiu, C., 2008. Ion beam acceleration in a diverging magnetic field. Applied Physics Letters 92 (191502), 1-3.

Biloiu, I. A., Scime, E. E., Biloiu, C., 2009. Laser induced fluoresence of xenon ions in a magnetized plasma. In: Proceedings of the XXIX International Conference on Phenomena in Ionized Gases. Cancun, Mexico.

Blackhall, L., Khachan, J., 2007. A simple electric thruster based on ion charge exchange. Journal of Physics D: Applied Physics 40, 2491-2494.

Block, L. P., 1978. A double layer review. Astrophysics and Space Science 55, 59-83.

Bohm, C., Perrin, J., 1993. Retarding-field energy analyzer for measurements of ion energy distributions and secondary emission coefficients in low-pressure radio frequency discharges. Review of Scientific Instruments 64 (1), 31-44.

Borisov, N., Mall, U., 2002. The structure of the double layer behind the Moon. Journal of Plasma Physics 61 (4), 277-299.

Boswell, R. W., 1970. Plasma Production Using a Standing Helicon Wave. Physics Letters A 33 (7), 457-458.

Boswell, R. W., 1984. Very Efficient Plasma Generation by Whistler Waves Near the Lower Hybrid Frequency. Plasma Physics and Controlled Fusion 26 (10), 1147-1162.

Boswell, R. W., Marsch, E., Charles, C., 2006. The current-free electric double layer in a coronal magnetic funnel. The Astrophysical Journal 640, L199-L202.

Boswell, R. W., Porteous, R. K., 1987. Large volume, high density rf inductively coupled plasma. Applied Physics Letters 50 (17), 1130-132.

Braithwaite, N. S. J., 2000. Introduction to gas discharges. Plasma Sources Science and Technology 9, 517-527.

Braithwaite, N. S. J., Franklin, R. N., 2009. Reflections on electrical probes. Plasma Sources Science and Technology 18 (014008), 1-6.

Breizman, B. N., Tushentsov, M. R., Arefiev, A. V., 2008. Magnetic nozzle and plasma detachment model for steady-state flow. Physics of Plasmas 15 (057103), 1-10.

Brophy, J., 2003. Advanced ion propulsion systems for affordable deep-space missions. Acta Astronautica 52, 309-316.

Buursink, J., 1998. Launch cost reduction by the reuse of the core stage of Ariane 5. Journal of Reducing Space Mission Cost 1, 209-223.

Byhring, H. S., Charles, C., Fredriksen, A., Boswell, R. W., 2008. Double layer in an expanding plasma: Simultaneous upstream and downstream measurements. Physics of Plasmas 15 (102113), 1-6. 
Cassady, L. D., Kodys, A. D., Choueiri, E. Y., 2002. Thermal Effects on Inverted Pendulum Thrust Stands for Steady-state High-power Plasma Thrusters. 38th AIAA/ASME/SAE/ASEE Joint Propulsion Conference (AIAA-2002-4118), 4118.

Chan, C., Hershkowitz, N., Lonngren, K. E., 1983. Electron temperature differences and double layers. Physics of Fluids 26 (6), 1587-1595.

Chang-Diaz, F. R., 2006. Plasma propulsion for interplanetary flight. Thin Solid Films 506-507, 449-453.

Charles, C., 1993. Ion energy distribution function in a multipole confined argon plasma diffusing from a 13.56-MHz helicon source. Journal of Vacuum Science and Technology A 11 (1), 157-163.

Charles, C., 2004. Hydrogen ion beam generated by a current-free electric double layer in a helicon plasma. Applied Physics Letters 84 (3), 332-334.

Charles, C., 2005a. High source potential upstream of a current-free electric double layer. Physics of Plasmas 12 (044508), 1-4.

Charles, C., 2005b. Spatially Resolved Energy Analyzer Measurements of an Ion Beam on the Low Potential Side of a Current-Free Double-Layer. IEEE Transactions on Plasma Science 33 (2), 336-337.

Charles, C., 2007. A review of recent laboratory double layer experiments. Plasma Sources Science and Technology 16 (4), R1-R25.

Charles, C., 2009. Plasmas for spacecraft propulsion. Journal of Physics D: Applied Physics 42 (163001), 1-18.

Charles, C., Boswell, R., 1998. Stress reduction in silicon dioxide layers by pulsing an oxygen/silane helicon diffusion plasma. Journal of Applied Physics 84 (1), 350-354.

Charles, C., Boswell, R., Alexander, P., Costa, C., Sutherland, O., Pfitzner, L., Franzen, R., Kingwell, J., Parfitt, A., Frigot, P.-E., Amo, J. D., Saccoccia, G., 2008. Operating the Helicon Double Layer Thruster in a space simulation chamber. IEEE Transactions on Plasma Science 36 (4, Part 1), 1196-1197.

Charles, C., Boswell, R. W., 2002. A Plasma Beam Generator. Australian Patent Application Number 2003232523 (PCT/AU2003/000763).

Charles, C., Boswell, R. W., 2003. Current-free double layer formation in a high-density helicon discharge. Applied Physics Letters 82 (9), 1356-1358.

Charles, C., Boswell, R. W., 2004a. Laboratory evidence of a supersonic ion beam generated by a current-free "helicon" double-layer. Physics of Plasmas 11 (4), 1706-1714.

Charles, C., Boswell, R. W., 2004b. Time development of a current-free double-layer. Physics of Plasmas 11 (8), 3808-3812.

Charles, C., Boswell, R. W., 2007. The magnetic-field-induced transition from an expanding plasma to a double layer containing expanding plasma. Applied Physics Letters 91 (201505), 1-3. 
Charles, C., Boswell, R. W., 2008. Effect of Exhaust Magnetic Field in a Helicon DoubleLayer Thruster Operating in Xenon. IEEE Transactions on Plasma Science 36 (5), 21412146.

Charles, C., Boswell, R. W., Bouchoule, A., Laure, C., Ranson, P., 1991. Plasma diffusion from a low pressure radio frequency source. Journal of Vacuum Science and Technology A 9 (3), 661-663.

Charles, C., Boswell, R. W., Cox, W., Laine, R., MacLellan, P., 2008a. Magnetic steering of a helicon double layer thruster. Applied Physics Letters 93 (201501), 1-3.

Charles, C., Boswell, R. W., Hawkins, R., 2009. Oblique Double Layers: A Comparison between Terrestrial and Auroral Measurements. Physical Review Letters 103 (095001), $1-4$.

Charles, C., Boswell, R. W., Laine, R., MacLellan, P., 2008b. An experimental investigation of alternative propellants for the helicon double layer thruster. Journal of Physics D: Applied Physics 41 (175213), 1-6.

Charles, C., Boswell, R. W., Lieberman, M. A., 2003. Energy balance in a low pressure capacitive discharge driven by a double-saddle antenna. Physics of Plasmas 10 (3), 891-899.

Charles, C., Boswell, R. W., Lieberman, M. A., 2006. Xenon ion beam characterization in a helicon double layer thruster. Applied Physics Letters 89 (261503), 1-3.

Charles, C., Boswell, R. W., Porteous, R. K., 1992. Measurement and modeling of ion energy distribution functions in a low pressure argon plasma diffusing from a 13.56 MHz helicon source. Journal of Vacuum Science \& Technology 10 (2), 398-403.

Charles, C., Degeling, A. W., Sheridan, T. E., Harris, J. H., Lieberman, M. A., Boswell, R. W., 2000. Absolute measurements and modelling of radio frequency electric fields using a retarding field energy analyzer. Physics of Plasmas 7 (12), 5232-5241.

Charles, C., Ramdutt, D., Brault, P., Caillard, A., Bulla, D., Boswell, R., Rabat, H., Dicks, A., 2007. Low energy plasma treatment of a proton exchange membrane used for low temperature fuel cells. Plasma Physics and Controlled Fusion 49, A73-A79.

Chavers, D. G., Chang-Diaz, F. R., 2002. Momentum flux measuring instrument for neutral and charged particle flows. Review of Scientific Instruments 73 (10), 3500-3507.

Chavers, D. G., Chang-Diaz, F. R., Irvine, C., Squire, J. P., 2006. Momentum and Heat Flux Measurements Using an Impact Target in Flowing Plasma. Journal of Propulsion and Power 22 (3), 637-644.

Cheetham, A. D., Rayner, J. P., 1998. Characterization and modeling of a Helicon plasma source. Journal of Vacuum Science and Technology A 16 (5), 2777-2784.

Chen, F., 1965. Electric Probes. In: Huddlestone, R. H., Leonard, S. L. (Eds.), Plasma Diagnostic Techniques. Academic Press, New York, Ch. 4, pp. 113-200. 
Chen, F. F., 2006. Physical mechanism of current-free double layers. Physics of Plasmas 13 (034502), 1-3.

Cohen, S. A., Siefert, N. S., Stange, S., Bolvin, R. F., Scime, E. E., Levinton, F. M., 2003. Ion acceleration in plasmas emerging from a helicon-heated magnetic-mirror device. Physics of Plasmas 10 (6), 2593-2598.

Cohen, S. A., Zonca, F., Timberlake, J., Bennett, T., Cuthbertson, J., Langer, W., Motley, R., 1990. An instrument for measuring the momentum flux from atomic and charged particle jets. Review of Scientific Instruments 61 (11), 3586-3591.

Collins, G. A., Tendys, J., 1994. Measurement of potentials and sheath formation in plasma immersion ion implantation. Journal of Vacuum Science and Technology B $12(2), 875-879$.

Conway, G. D., Perry, A. J., Boswell, R. W., 1998. Evolution of ion and electron energy distributions in pulsed plasma discharges. Plasma Sources Science and Technology 7 , 337-347.

Corr, C. S., Boswell, R. W., Charles, C., Zanger, J., 2008. Spatial evolution of an ion beam created by a geometrically expanding low-pressure argon plasma. Applied Physics Letters 92 (221508), 1-3.

Corr, C. S., Zanger, J., Boswell, R. W., Charles, C., 2007. Ion beam formation in a lowpressure geometrically expanding argon plasma. Applied Physics Letters 91 (241501), $1-3$.

Cox, W., Charles, C., Boswell, R. W., Hawkins, R., 2008a. Spatial retarding field energy analyzer measurements downstream of a helicon double layer plasma. Applied Physics Letters 93 (071505), 1-3.

Cox, W., Hawkins, R., Charles, C., Boswell, R. W., 2008b. Three-Dimensional Mapping of Ion Density in a Double-Layer Helicon Plasma. IEEE Transactions on Plasma Science 36 (4), 1386-1387.

Cubbin, E. A., Ziemer, J. K., Choueiri, E. Y., Jahn, R. G., 1997. Pulsed thrust measurements using laser interferometry. Review of Scientific Instruments 68 (6), 2339-2346.

Degeling, A. W., Jung, C. O., Boswell, R. W., Ellingboe, A. R., 1996. Plasma production from helicon waves. Physics of Plasmas 3 (7), 2788-2796.

Deline, C. A., Bengston, R. G., Breizman, B. N., Tushentsov, M. R., Jones, J. E., Chavers, D. G., Dobson, C. C., Schuettpelz, B. M., 2009. Plume detachment from a magnetic nozzle. Physics of Plasmas 16 (033502), 1-9.

Ergun, R. E., Andersson, L., Tao, J., Angelopoulos, V., Bonnell, J., McFadden, J. P., Larson, D. E., Eriksson, S., Johansson, T., Cully, C. M., Newman, D. N., Goldman, M. V., Roux, A., LeContel, O., Glasmeier, K.-H., Baumjohann, W., 2009. Observations of Double Layers in Earth's Plasma Sheet. Physical Review Letters 102 (155002), 1-5.

Fitz, C., Fukarek, W., Kolitsch, A., Moller, W., 2000. An instrument for in-situ stress measurement in thin films during growth. Surface \& Coatings Technology 128, 474-478. 
Franck, C. M., Grulke, O., Klinger, T., 2003. Mode transitions in helicon discharges. Physics of Plasmas 10 (1), 323-325.

Frisbee, R. H., 2003. Advanced Space Propulsion for the 21st Century. Journal of Propulsion and Power 19 (6), 1129-1154.

Fruchtman, A., 2006. Electric Field in a Double Layer and the Imparted Momentum. Physical Review Letters 96 (065002), 1-4.

Gahan, D., Dolinaj, B., Hopkins, M. B., 2008. Comparison of plasma parameters determined with a Langmuir probe and with a retarding field energy analyzer. Plasma Sources Science and Technology 17 (035026), 1-9.

Gamero-Castano, M., 2003. A torsional balance for the characterization of microNewton thrusters. Review of Scientific Instruments 74 (10), 4509-4512.

Gesto, F. N., Blackwell, B. D., Charles, C., Boswell, R. W., 2006. Ion Detachment in the Helicon Double-Layer Thruster Exhaust Beam. Journal of Propulsion and Power 22 (1), 24-30.

Gesto, F. N., Charles, C., Boswell, R. W., 2008. Xenon Ion Beam Detachment From a Helicon Double Layer Thruster . IEEE Transactions on Plasma Science 36 (4), 11941195.

Godyak, V. A., Piejak, R. B., Alexandrovich, B. M., 2002. Electron energy distribution function measurements and plasma parameters in inductively coupled argon plasma. Plasma Sources Science and Technology 11 (4), 525-543.

Goebel, D. M., Katz, I., 2008. Fundamentals of Electric Propulsion: Ion and Hall Thrusters. JPL Space Science and Technology Series. John Wiley \& Sons, Hoboken, New Jersey.

Goncharov, S. F., Pashinin, P. P., Perov, V. Y., Serov, R. V., Yanovsky, V. P., 1988. Hollow ballistic pendulum for plasma momentum measurements. Review of Scientific Instruments 59 (5), 709-711.

Goswami, K. S., Saharia, K., Schamel, H., 2008. Theory of current-free double layers in plasmas. Physics of Plasmas 15 (062111), 1-6.

Grun, J., Ripin, B. H., 1982. Ballistic pendula for measuring the momentum of a laserproduced plasma. Review of Scientific Instruments 53 (12), 1878-1881.

Haag, T. W., 1991. Thrust stand for high-power electric propulsion devices. Review of Scientific Instruments 62 (5), 1186-1191.

Hairapetian, G., Stenzel, R. L., 1988. Expansion of a Two-Electron-Population Plasma into Vacuum. Physical Review Letters 61 (14), 1607-1610.

Hall, D. F., Newman, B. E., Womack, J. R., 1970. Electrostatic Rocket Exhaust Effects on Solar-Electric Spacecraft Subsystems. Journal of Spacecraft and Rockets 7, 305.

Hamberger, S. M., Blackwell, B. D., Sharp, L. E., Shenton, D. B., 1990. H-1 design and construction. Fusion Technology 17 (1), 123-130. 
Harper, J. M. E., Cuomo, J. J., Leary, P. A., Summa, G. M., Kaufman, H. R., Bresnock, F. J., 1981. Low Energy Ion Beam Etching. Journal of The Electrochemical Society 128 (5), 1077-1083.

Hatakeyama, R., Suzuki, Y., Sato, N., 1983. Formation of Electrostatic Potential Barrier between Different Plasmas. Physical Review Letters 50 (16), 1203-1206.

Hershkowitz, N., 1985. Review of recent laboratory double layer experiments. Space Science Reviews 41, 351-391.

Hershkowitz, N., 1989. How Langmuir Probes Work. In: Auciello, O., Flamm, D. L. (Eds.), Plasma Diagnostics. Vol. 1. Academic Press, New York, Ch. 3.

Holkeboer, D. H., Jones, D. W., Pagano, F., Santeler, D. J., 1967. Vacuum Technology and Space Simulation. American Vacuum Society Classics of Vacuum Science and Technology. American Institute of Physics, New York.

Hooper, E. B., 1993. Plasma Detachment from a Magnetic Nozzle. Journal of Propulsion and Power 9 (5), 757-763.

Hopman, H. J., Jurgens, B., van Wakeren, J. H. A., Ficke, H. G., 1977. The construction of a retarding field energy analyser. Journal of Physics E: Scientific Instruments 10 (3), 287-292.

Ingram, S. G., Braithwaite, N. S. J., 1988. Ion and electron energy analysis at a surface in an RF discharge. Journal of Physics D: Applied Physics 21, 1496-1503.

Inutake, M., Ando, A., Hattori, K., Tobari, H., Makita, T., Shibata, M., Kasashima, Y., Komagome, T., 2007. Generation of supersonic plasma flows using an applied-field MPD arcjet and ICRF heating. Plasma Physics and Controlled Fusion 49 (5A, Sp. Iss. SI), A121-A134.

Jahn, R. G., 1968. Physics of Electric Propulsion. McGraw-Hill, New York.

Jahn, R. G., Choueiri, E. Y., 2002. Electric Propulsion. In: Encyclopedia of Physical Sciences and Technology, 3rd Edition. Vol. 5. Academic Press, San Diego, pp. 125-141.

Jamison, A. J., Ketsdever, A. D., Muntz, E. P., 2002. Gas dynamic calibration of a nanoNewton thrust stand. Review of Scientific Instruments 73 (10), 3629-3637.

Jaworski, M. A., Jurczyk, B. E., Antonsen, E. L., Ruzic, D. N., 2006. Direct current magnetic insulation of an immersed RF antenna. Plasma Sources Science and Technology $15,474-478$.

Keese, A. M., Scime, E. E., Charles, C., Meige, A., Boswell, R. W., 2005. The ion velocity distribution function in a current-free double layer. Physics of Plasmas 12 (093502), $1-7$.

Kemp, M. A., Kovaleski, S. D., 2008. Thrust measurements of the ferroelectric plasma thruster. IEEE Transactions on Plasma Science 36 (2, Part 1), 356-362. 
Kodys, A. D., Cassady, L. D., Choueiri, E. Y., 2003. Thermal Effects on Inverted Pendulum Thrust Stands for Steady-state High-power Plasma Thrusters. In: 39th AIAA/ASME/SAE/ASEE Joint Propulsion Conference. No. AIAA-2003-4842. American Institute of Aeronautics \& Astronautics, pp. 1-7.

Kodys, A. D., Murray, R., Cassady, L. D., Choueiri, E. Y., 2006. An Inverted Pendulum Thrust Stand for High-Power Electric Thrusters. 42nd AIAA/ASME/SAE/ASEE Joint Propulsion Conference (AIAA-2006-4821), 4821.

Lafleur, T., Charles, C., Boswell, R. W., 2009. Detailed plasma potential measurements in a radio-frequency expanding plasma obtained from various electrostatic probes. Physics of Plasmas 16 (044510).

Lafleur, T., Charles, C., Boswell, R. W., 2010. Ion beam formation in a very low magnetic field expanding helicon discharge. Physics of Plasmas in press.

Larson, W. J., Wertz, J. R. (Eds.), 1999. Space Mission Analysis and Design, 3rd Edition. Space Technology Series. Microcosm Press, El Segundo, California.

Lee, Y., Kudva, G. N., Litzinger, T. A., 2000. A facility for solid-propellant response measurements under pressure-driven conditions. Measurement Science and Technology $11(1), 51-58$.

Li, J., Tang, Z., 2005. Laser micro-impulse torsion pendulum. Chinese Optical Letters $3(2), 76-79$.

Lieberman, M. A., Boswell, R. W., 1998. Modeling the transitions from capacitive to inductive to wave-sustained rf discharges. Journal De Physique IV France 8 (Pr7), 145164.

Lieberman, M. A., Charles, C., 2006. Theory for Formation of a Low-Pressure, CurrentFree Double Layer. Physical Review Letters 97 (045003), 1-4.

Lieberman, M. A., Charles, C., Boswell, R. W., 2006. A theory for formation of a low pressure, current-free double layer. Journal of Physics D: Applied Physics 39, 32943304.

Lieberman, M. A., Litchtenberg, A. J., 2005. Principles of Plasma Discharges and Materials Processing, 2nd Edition. Wiley-Interscience, New York.

Longmier, B., Hershkowitz, N., 2008. Improved operation of the nonambipolar electron source. Review of Scientific Instruments 79 (093506).

Longmier, B. W., Reid, B. M., Gallimore, A. D., Chang-Diaz, F. R., Squire, J. P., Glover, T. W., Chavers, G., III, E. A. B., 2009. Validating a Plasma Momentum Flux Sensor to an Inverted Pendulum Thrust Stand. Journal of Propulsion and Power 25 (3), 746-762.

Lunt, T., Silva, C., Fernandes, H., Hidalgo, C., Pedrosa, M. A., Duarte, P., Figueiredo, H., Pereira, T., 2007. Edge plasma pressure measurements using a mechanical force sensor on the tokamak ISTTOK. Plasma Physics and Controlled Fusion 49, 1783-1790. 
Makrinich, G., Fruchtman, A., 2009. Experimental study of a radial plasma source. Physics of Plasmas 16 (043507), 1-8.

Martinez-Sanchez, M., Pollard, J. E., 1998. Spacecraft Electric Propulsion-An Overview. Journal of Propulsion and Power 14 (5), 688-699.

Meige, A., 2006. Numerical modeling of low-pressure plasmas: applications to electric double layers. Ph.D. thesis, The Australian National University.

Meige, A., Boswell, R. W., Charles, C., Boeuf, J.-P., Hagelaar, G., Turner, M. M., 2005a. One-Dimensional Simulation of an Ion Beam Generated by a Current-Cree Double Layer. IEEE Transactions on Plasma Science 33 (2), 334-335.

Meige, A., Boswell, R. W., Charles, C., Turner, M. M., 2005b. One-dimensional particlein-cell simulation of a current-free double layer in an expanding plasma. Physics of Plasmas 12 (052317), 1-10.

Meige, A., Plihon, N., Hagelaar, G. J. M., Boeuf, J.-P., Chabert, P., Boswell, R. W., 2007. Propagating double layer in electronegative plasmas. Physics of Plasmas 14 (053508), $1-11$.

Mott-Smith, H. M., Langmuir, I., 1926. The Theory of Collectors in Gaseous Discharges. Physical Review 28 (4), 727-763.

Mozer, F. S., Carlson, C. W., Hudson, M. K., Torbert, R. B., Parady, B., Yatteau, J., 1977. Observations of Paired Electrostatic Shocks in the Polar Magnetosphere. Physical Review Letters 38, 292-295.

Nagao, N., Yokota, S., Komurasaki, K., Arakawa, Y., 2007. Development of a twodimensional dual pendulum thrust stand for Hall thrusters. Review of Scientific Instruments 78 (115108), 1-4.

Nedzelskiy, I. S., Silva, C., Fernandes, H., Duarte, P., Varandas, C. A. F., 2007. Compact cantilever force probe for plasma pressure measurements. Review of Scientific Instruments 78 (123505), $1-6$.

Neiswender, D. D., Kohout, F. C., 1972. An Apparatus for Electron Energy Analysis in Glow Discharge Plasmas. Review of Scientific Instruments 43 (10), 1475-1480.

Niemi, K., Krämer, M., 2008. Helicon mode formation and radio frequency power deposition in a helicon-produced plasma. Physics of Plasmas 15 (073503).

Perkins, F. W., Sun, Y. C., 1981. Double Layers without Current. Physical Review Letters $46,115-118$.

Perry, A., Conway, G., Boswell, R., Persing, H., 2002. Modulated plasma potentials and cross field diffusion in a Helicon plasma. Physics of Plasmas 9 (7), 3171-3177.

Perry, A. J., Vender, D., Boswell, R. W., 1991. The application of the helicon source to plasma processing. Journal of Vacuum Science and Technology B 9 (2, Part 1), 310-317. 
Phipps, C. R., Luke, J. R., Helgeson, W., Johnson, R., 2006. A low-noise thrust stand for microthrusters with $25 \mathrm{nN}$ resolution. In: Komurasaki, $\mathrm{K}$ and Yabe, $\mathrm{T}$ and Uchida, $\mathrm{S}$ and Sasoh, A (Ed.), Beamed Energy Propulsion: Fourth International Symposium on Beamed Energy Propulsion. Vol. 830 of AIP Conference Proceedings. pp. 492-499.

Plihon, N., Chabert, P., Corr, C. S., 2007. Experimental investigation of double layers in expanding plasmas. Physics of Plasmas 14 (013506), 1-16.

Plihon, N., Corr, C. S., Chabert, P., Raimbault, J. L., 2005. Periodic formation and propagation of double layers in the expansion chamber of an inductive discharge operating in Ar/SF6 mixtures. Journal of Applied Physics 98 (023306), 1-7.

Polzin, K. A., Markusic, T. E., Stanojev, B. J., DeHoyos, A., Spaun, B., 2006. Thrust stand for electric propulsion performance evaluation. Review of Scientific Instruments 77 (105108), 1-9.

Popescu, S., Ohtsu, Y., Fujita, H., 2006. Current-free double-layer formation in inductively coupled plasma in a uniform magnetic field. Physical Review E 73 (066405), 1-8.

Raadu, M. A., 1989. The physics of double layers and their role in astrophysics. Physics Reports 178 (2), 25-97.

Rayman, M. D., Fraschetti, T. C., Raymond, C. A., Russell, C. T., 2006. Dawn: A mission in development for exploration of main belt asteroids Vesta and Ceres. Acta Astronautica $58,605-616$.

Rayner, J. P., Cheetham, A. D., 1999. Helicon modes in a cylindrical plasma source. Plasma Sources Science and Technology 8, 79-87.

Rayner, J. P., Cheetham, A. D., French, G. N., 1996. Radio frequency matching for helicon plasma sources. Journal of Vacuum Science and Technology A 14 (4), 2048-2055.

Sasoh, A., Arakawa, Y., 1993. A high-resolution thrust stand for ground tests of lowthrust space propulsion devices. Review of Scientific Instruments 64 (3), 719-723.

Savitzky, A., Golay, M. J. E., 1964. Smoothing and Differentiation of Data by Simplified Least Squares Procedures. Analytical Chemistry 36 (8), 1627-1639.

Scharer, J., Degeling, A., Borg, G., Boswell, R., 2002. Measurements of helicon wave propagation and Ar II emission. Physics of Plasmas 9 (9), 3734-3742.

Schmidt, T. D., Sebolt, W., Auweter-Kurtz, M., 2006. Flexible Piloted Mars Missions Using Continuous Electric Propulsion. Journal of Spacecraft and Rockets 43 (6), 12311238.

Schmit, P. F., Fisch, N. J., 2009. Magnetic detachment and plume control in escaping magnetized plasma. Journal of Plasma Physics 75 (3), 359-371.

Scime, E. E., Keesee, A. M., Boswell, R. W., 2008. Mini-conference on helicon plasma sources. Physics of Plasmas 15 (058301).

Sefkow, A. B., Cohen, S. A., 2009. Particle-in-cell modeling of magnetized argon plasma flow through small mechanical apertures. Physics of Plasmas 16 (053501), 1-13. 
Shamrai, K. P., Virko, Y. V., Virko, V. R., Yakimenko, A. I., 2006. Compact Helicon Plasma Source with Permanent Magnets for Electric Propulsion Application. In: 42nd AIAA/ASME/SAE/ASEE Joint Propulsion Conference. No. 2006-4845. pp. 1-7.

Sheridan, T. E., 2000. How big is a small Langmuir probe? Physics of Plasmas 7 (7), 3084-3088.

Stephen, R. J., Rajanna, K., Dhar, V., Kalyan-Kumar, K. G., Nagabushanam, S., 2004. Thin-Film Strain Gauge Sensors for Ion Thrust Measurement. IEEE Sensors Journal 4 (3), 373-377.

Stephen, R. J., Rajanna, K., Dhar, V., Kumar, K. G. K., Nagabushanam, S., 2001. Strain gauge based thrust measurement system for a stationary plasma thruster. Measurement Science and Technology 12, 1568-1575.

Sudit, I. D., Chen, F. F., 1994. RF compensated probes for high-density discharges. Plasma Sources Science and Technology 3, 162-168.

Sugai, H., Nakamura, K., Suzuki, K., 1994. Electrostatic Coupling of Antenna and the Shielding Effect in Inductive RF Plasmas. Japanese Journal of Applied Physics 33, 2189-2193.

Sun, X., Biloiu, C., Hardin, R., Scime, E. E., 2004. Parallel velocity and temperature of argon ions in an expanding, helicon source driven plasma. Plasma Sources Science and Technology 13, 359-370.

Sun, X., Cohen, S. A., Scime, E. E., Miah, M., 2005a. On-axis parallel ion speeds near mechanical and magnetic apertures in a helicon plasma device. Physics of Plasmas 12 (103509), 1-8.

Sun, X., Keese, A. M., Biloiu, C., Scime, E. E., Meige, A., Charles, C., Boswell, R. W., 2005b. Observations of Ion-Beam Formation in a Current-Free Double Layer. Physical Review Letters 95 (025004), 1-4.

Sutherland, O., Charles, C., Plihon, N., Boswell, R. W., 2005. Experimental Evidence of a Double Layer in a Large Volume Helicon Reactor. Physical Review Letters 95 (205002), $1-4$.

Sutton, G. P., Biblarz, O., 2001. Rocket Propulsion Elements, 7th Edition. John Wiley \& Sons, New York.

Suzuki, K., Nakamura, K., Ohkubo, H., Sugai, H., 1998. Power transfer efficiency and mode jump in an inductive RF discharge. Plasma Sources Science and Technology $7(1), 13-20$.

Takahashi, K., Charles, C., Boswell, R., Cox, W., Hatakeyama, R., 2009. Transport of energetic electrons in a magnetically expanding helicon double layer plasma. Applied Physics Letters 94 (191503), 1-3.

Takahashi, K., Charles, C., Boswell, R. W., Kaneko, T., Hatakeyama, R., 2007. Measurement of the energy distribution of trapped and free electrons in a current-free double layer. Physics of Plasmas 14 (114503), 1-4. 
Takahashi, K., Fujiwara, T., 2009. Observation of weakly and strongly diverging ion beams in a magnetically expanding plasma. Applied Physics Letters 94 (061502), 13.

Takahashi, K., Kaneko, T., Hatakeyama, R., 2008a. Double layer created by electron cyclotron resonance heating in an inhomogeneously magnetized plasma with highspeed ion flow. Physics of Plasmas 15 (072108), 1-5.

Takahashi, K., Oguni, K., Yamada, H., Fujiwara, T., 2008b. Ion acceleration in a solenoidfree plasma expanded by permanent magnets. Physics of Plasmas 15 (084501), 1-4.

Tribble, A. C., 2003. The Space Environment: Implications for Spacecraft Design. Princeton University Press, Princeton, New Jersey.

Tsiolkovsky, K. E., 1903. The Investigation of Space by Means of Reactive Devices (in Russian). Nauchnoe Obozrenie 5 (3).

Turner, M. J. L., 2005. Rocket and Spacecraft Propulsion: Principles, Practice and New Developments, 2nd Edition. Springer-Praxis, Chichester, UK.

Uematsu, K., Morimoto, S., Kuriki, K., 1985. MPD Thruster Performance with Various Propellants. Journal of Spacecraft and Rockets 22 (4), 412-416.

West, M. D., Charles, C., Boswell, R. W., 2008. Testing a Helicon Double Layer Thruster Immersed in a Space-Simulation Chamber. Journal of Propulsion and Power 24 (1), 134-141.

West, M. D., Charles, C., Boswell, R. W., 2009a. High density mode in xenon produced by a Helicon Double Layer Thruster. Journal of Physics D: Applied Physics 42 (245201), $1-9$.

West, M. D., Charles, C., Boswell, R. W., 2009b. A high sensitivity momentum flux measuring instrument for plasma thruster exhausts and diffusive plasmas. Review of Scientific Instruments 80 (053509), 1-9.

West, M. D., Charles, C., Boswell, R. W., 2010. Operating RF Antennas in Vacuum: Implications for Ground Testing Plasma Thrusters. Journal of Propulsion and Power in review.

West, M. D., Lee, P., 2007. Human Exploration of Phobos \& Deimos: Engineering Challenges \& Unique Opportunities. In: 1st International Conference on the Exploration of Phobos and Deimos. No. 7032. NASA Ames, Moffet Field, California, p. 1.

Wilson, M. J., Bushman, S. S., Burton, R. L., 1997. A Compact Thrust Stand for Pulsed Plasma Thrusters. In: Proceedings of the 25th International Electric Propulsion Conference. No. IEPC-97-122. Electric Rocket Propulsion Society, Cleveland, OH, pp. 765772.

Yang, T. F., Liu, P., Chang-Diaz, F. R., Lander, H., Childs, R. A., Becker, H. D., Fairfax, S. A., 1995. A double pendulum plasma thrust balance and thrust measurement at a tandem mirror exhaust. Review of Scientific Instruments 66 (9), 4637-4643. 
Yin, Y., Bilek, M. M., McKenzie, D. R., Boswell, R. W., Charles, C., 2004. Micro-arcing in radio frequency plasmas. Journal of Physics D: Applied Physics 37, 2871-2875.

Yin, Y., Bilek, M. M., McKenzie, D. R., Boswell, R. W., Charles, C., 2005. Microarcing instability in RF PECVD plasma system. Surface \& Coatings Technology 198, 379-383.

Yin, Y., McKenzie, D. R., Bilek, M. M., 2006. Analytic analysis on asymmetrical micro arcing in high plasma potential rf plasma systems. Plasma Sources Science and Technology 15, 99-104.

Yoshikawa, T., Kagaya, Y., Kuriki, K., 1984. Thrust and Efficiency of the K-III MPD Thruster. Journal of Spacecraft and Rockets 21 (5), 481-487.

Ziemba, T., Euripides, P., Slough, J., Winglee, R., Giersch, L., Carscadden, J., Schnackenberg, T., Isley, S., 2006. Plasma characteristics of a high power helicon discharge. Plasma Sources Science and Technology 15 (3), 517-525. 